Supporting Information for

\title{
Catalytic, Enantioselective 1,2-Difluorination of Cinnamamides
}

Moriana K. Haj, Steven M. Banik, and Eric N. Jacobsen*

Department of Chemistry and Chemical Biology, Harvard University, Cambridge, Massachusetts 02138, United States

\section{Table of Contents}

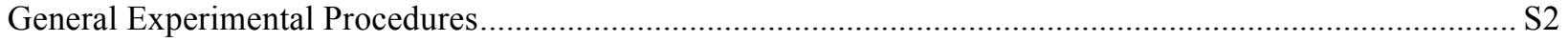

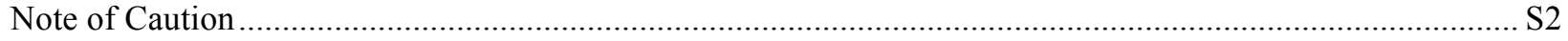

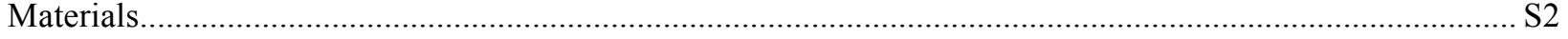

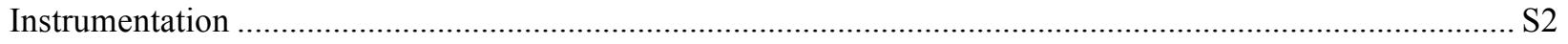

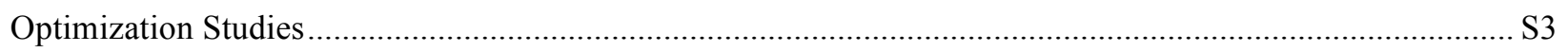

Preparation and Characterization of Catalysts and Substrates....................................................................... S5

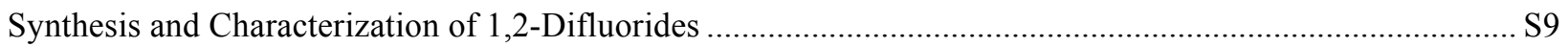

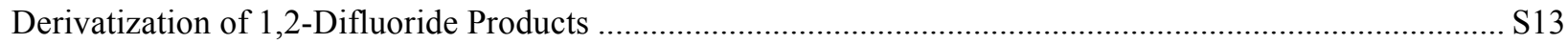

Measurement of Product Ratios for Selected Cinnamamides...................................................................... S15

Correlations Between Product Ratios and Steric and Electronic Parameters for Amide $N$-Substituents ......... S16

Chiral GC and HPLC Traces of 1,2-Difluoride Products and Derivatives .................................................... S19

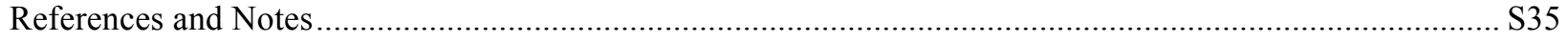

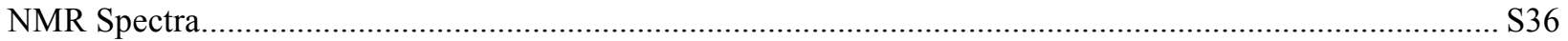




\section{General Experimental Procedures}

All reactions for the preparation of substrates and catalysts were performed in standard, dry glassware fitted with rubber septa under an inert atmosphere of nitrogen unless otherwise described. All difluorination reactions were performed in polyethylene tubes sealed with a high density polyethylene cap under an atmosphere of air. Stainless steel gas-tight syringes or cannulae were used to transfer air- and moisturesensitive liquids. Reported concentrations refer to solution volumes at room temperature. Concentration of organic solutions under reduced pressure was performed using house vacuum (ca. $40 \mathrm{~mm} \mathrm{Hg}$ ) at $30{ }^{\circ} \mathrm{C}$. Column chromatography was performed with SiliaFlash ${ }^{\circledR}$ P60 $(40-63 \mu \mathrm{m}, 60 \AA)$ silica gel from Silicycle. Thin layer chromatography (TLC) was used for reaction monitoring, and product detection was performed using pre-coated glass plates covered with $0.20 \mathrm{~mm}$ silica gel with fluorescent indicator; plates were visualized by exposure to UV light $(\lambda=254 \mathrm{~nm})$ or by staining with potassium permanganate or ninhydrin.

\section{Note of Caution}

Pyridine $9 \mathrm{HF}$ is a corrosive and toxic substance that will corrode glassware. Safe handling can be conducted with plastic syringes and metal needles, with $\mathrm{NaHCO}_{3}$ (aq.) or $\mathrm{NaOH}$ (aq.) employed to quench excess $\mathrm{HF}$. Though reactions should not be conducted in glassware when employing pyridine $\bullet \mathrm{HF}$, glassware may be used to quench reactions provided sufficient quantities of base are present. Always handle pyridine $9 \mathrm{HF}$ while wearing gloves and in a fume hood. As a precautionary measure, have calcium gluconate gel nearby and apply immediately and liberally on skin exposed to pyridine $9 \mathrm{HF}$.

\section{Materials}

Reagents were purchased in reagent grade from commercial suppliers and used as received, unless otherwise described. Anhydrous solvents (benzene, dichloromethane, diethyl ether, $N, N$-dimethylformamide, tetrahydrofuran, and toluene) were prepared by passing the solvent through an activated alumina column. Triethylamine and diisopropylethylamine were distilled over calcium hydride at atmospheric pressure.

\section{Instrumentation}

Proton nuclear magnetic resonance ( ${ }^{1} \mathrm{H}$ NMR) spectra were recorded on an Inova-500 spectrometer, are reported in parts per million downfield from tetramethylsilane, and are referenced to the residual protium resonances of the NMR solvent $\left(\mathrm{CDCl}_{3}: 7.26\left[\mathrm{CHCl}_{3}\right]\right.$, acetone-d6: 2.05 [acetone-d5], DMSO-d6: 2.50 [DMSO-d5]). Proton-decoupled carbon-13 nuclear magnetic resonance (13C $\{1 \mathrm{H}\}$ NMR) spectra were recorded on an Inova-500 spectrometer, are reported in parts per million downfield from tetramethylsilane, and are referenced to the carbon resonances of the NMR solvent (CDCl3: 77.23, acetone-d6: 29.92, DMSO-d6: 39.61). Chemical shifts for fluorine-19 nuclear magnetic resonance (19F NMR) were recorded on an Inova500 spectrometer, are reported in parts per million downfield from chlorotrifluoromethane, and are referenced to the fluorine resonance of chlorotrifluoromethane $(\delta=0)$. Data are represented as follows: chemical shift, multiplicity $(\mathrm{br}=$ broad, $\mathrm{s}=$ singlet, $\mathrm{d}=$ doublet, $\mathrm{t}=$ triplet, $\mathrm{q}=$ quartet, quin $=$ quintet, $\mathrm{sept}=$ septet, $\mathrm{m}=$ multiplet), coupling constants in Hertz (Hz), integration.

Infrared spectra were recorded using a Bruker Tensor 27 FT-IR spectrometer.

High-resolution mass spectrometric data were obtained on an Agilent 6210 time-of-flight HPLC/MS spectrometer (ESI-TOF).

Chiral GC analysis was performed using an Agilent 7890A GC system using commercially available columns. Chiral HPLC analysis was performed using an Agilent 1200 series quaternary HPLC system using commercially available CHIRALCEL analytical columns (4.6 x $250 \mathrm{~mm})$.

Optical rotations were measured using a $1 \mathrm{~mL}$ cell with a $5 \mathrm{~cm}$ path length on a Jasco P-2000 digital polarimeter. 


\section{Optimization Studies}

\section{General procedure for optimization of $\mathrm{N}$-alkyl group and HF loading}

Catalyst $1 \mathrm{~b}(15.4 \mathrm{mg}, 20.0 \mu \mathrm{mol}, 20.0 \mathrm{~mol} \%)$, cinnamamide (100 $\mu \mathrm{mol}, 1.00$ equiv), and dichloromethane $(200 \mu \mathrm{L})$ were combined in a polyethylene tube at room temperature. Pyridinium poly(hydrogen fluoride) (pyr $\bullet \mathrm{HF}, 70 \%$ hydrogen fluoride by weight, $260 \mu \mathrm{L}, 100$ equiv hydrogen fluoride) was added via syringe at $78{ }^{\circ} \mathrm{C}$, followed by $m$-chloroperbenzoic acid ( $m$ CPBA, $77 \%$ by weight, $29.1 \mathrm{mg}, 1.30$ equiv). The reaction was warmed to $4{ }^{\circ} \mathrm{C}$ and stirred for $24 \mathrm{~h}$ at that temperature. The reaction was diluted with dichloromethane and poured slowly into a slurry of basic alumina in dichloromethane at $-78{ }^{\circ} \mathrm{C}$. The reaction was warmed to room temperature with stirring, filtered, and concentrated under reduced pressure. The crude product mixture was analyzed by ${ }^{1} \mathrm{H}$ NMR using nitrobenzene as an internal standard to determine the yield of product, by ${ }^{19} \mathrm{~F}$ NMR to determine the product ratio, and by chiral GC to determine the enantiomeric excess.

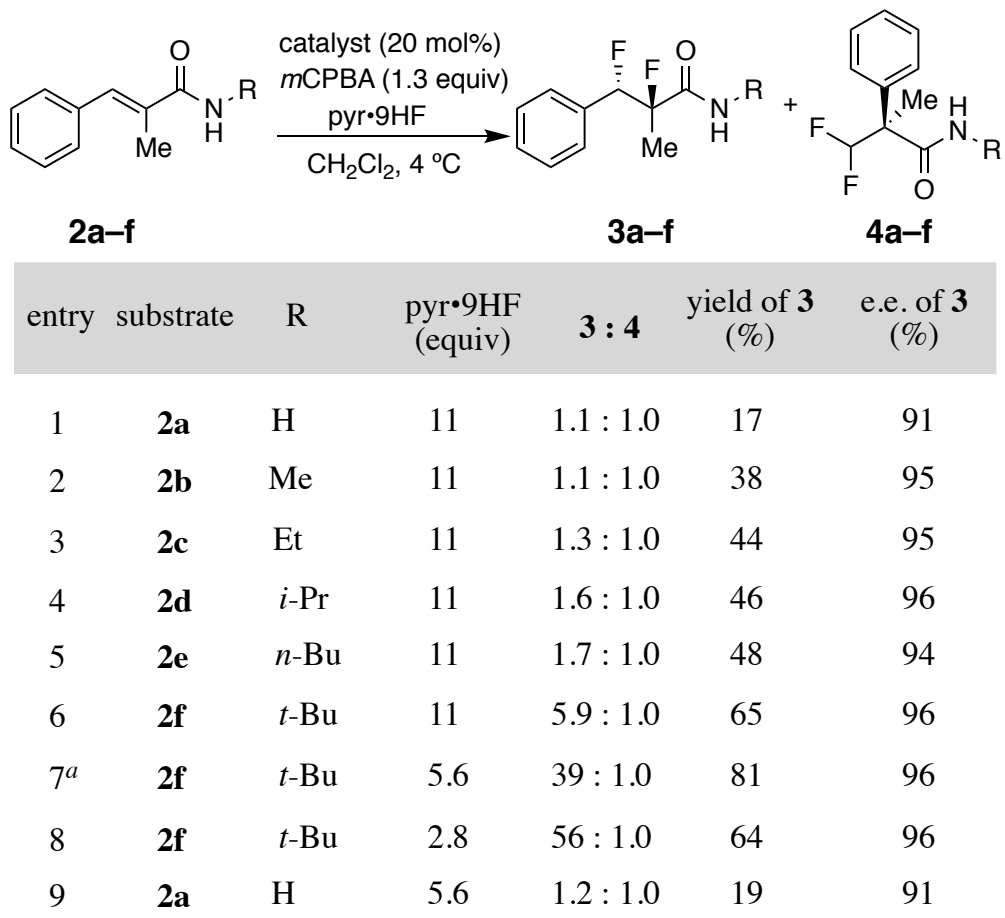

Table S1. Optimization of $N$-alkyl group and HF loading. Unless otherwise noted, reactions were performed on 0.10 mmol scale, with yields of 1,2-difluoride determined by ${ }^{1} \mathrm{H}$ NMR using nitrobenzene as an internal standard. Reported ratios of 1,2-difluoride to 1,1-difluoride were determined by ${ }^{19} \mathrm{~F}$ NMR analysis of crude product mixtures. ${ }^{a}$ Reaction was conducted on $1.00 \mathrm{mmol}$ scale, and the isolated yield of $\mathbf{3}$ is listed.

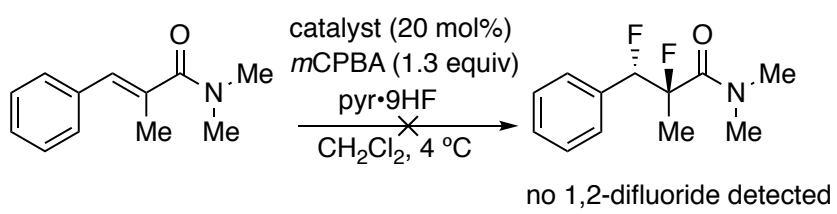




\section{General procedure for catalyst optimization}

Catalyst (20.0 $\mu \mathrm{mol}, 20.0 \mathrm{~mol} \%)$, cinnamamide $2 \mathrm{f}(21.7 \mathrm{mg}, 100 \mu \mathrm{mol}, 1.00$ equiv), and dichloromethane $(200 \mu \mathrm{L})$ were combined in a polyethylene tube at room temperature. Pyridinium poly(hydrogen fluoride) (pyr $\bullet$ HF, $70 \%$ hydrogen fluoride by weight, 30 or 50 equiv hydrogen fluoride) was added via syringe at -78 ${ }^{\circ} \mathrm{C}$, followed by $m$-chloroperbenzoic acid ( $\mathrm{mCPBA}, 77 \%$ by weight, $29.1 \mathrm{mg}, 1.30$ equiv). The reaction was warmed to $4{ }^{\circ} \mathrm{C}$ or room temperature and stirred for $24 \mathrm{~h}$ at that temperature. The reaction was diluted with dichloromethane and poured slowly into a slurry of basic alumina in dichloromethane at $-78{ }^{\circ} \mathrm{C}$. The reaction was warmed to room temperature with stirring, filtered, and concentrated under reduced pressure. The crude product mixture was analyzed by ${ }^{1} \mathrm{H}$ NMR using nitrobenzene as an internal standard to determine the yield of product, by ${ }^{19} \mathrm{~F}$ NMR to determine the product ratio, and by chiral GC to determine the enantiomeric excess.

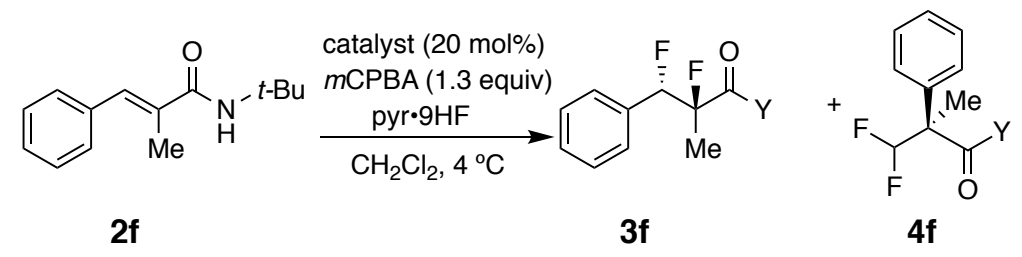

$\begin{array}{cccccc}\text { entry } & \text { catalyst } & \begin{array}{c}\text { pyr・9HF } \\ (\text { equiv) }\end{array} & \begin{array}{c}\mathrm{T}\left({ }^{\circ} \mathrm{C}\right) \\ \begin{array}{c}\text { yield of } \mathbf{3} \\ (\%)\end{array}\end{array} & \begin{array}{c}\text { e.e. of } \mathbf{3} \\ (\%)\end{array} \\ 1^{b} & \mathbf{1 a} & 5.6 & 4 & 78 & 96 \\ 2^{a, b} & \mathbf{1 b} & 5.6 & 4 & 81 & 96 \\ 3 & \mathbf{S - 1} & 5.6 & 4 & 67 & 68 \\ 4 & \mathbf{1 b} & 3.4 & \mathrm{rt} & 69 & 91 \\ 5 & \mathbf{S - 2} & 3.4 & \mathrm{rt} & 42 & -48 \\ 6 & \mathbf{S - 3} & 3.4 & \mathrm{rt} & 38 & 11 \\ 7 & \mathbf{S}-4 & 3.4 & \mathrm{rt} & 33 & 41 \\ 8 & \mathbf{S}-5 & 3.4 & \mathrm{rt} & 41 & -9\end{array}$<smiles>CCCC(=O)OC(Br)C(=O)Oc1cc(C(=O)O)cc(OC(Br)C(=O)O)c1I</smiles>

1a: $\mathrm{R}=\mathrm{Me}$<smiles>COC(=O)c1cc(OC(=O)C(=O)OCc2ccccc2)c(I)c(OC(=O)C(=O)OCc2ccccc2)c1</smiles>

S-1<smiles>COC(=O)C(CC1CCCCC1)Oc1cc(OC(C)=O)c(I)c(OC(CC2CCCCC2)C(=O)OC)c1</smiles>

S-2

1b: $\mathrm{R}=\mathrm{Bn}$

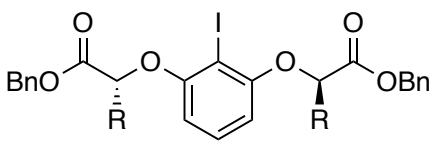

S-3: $R=M e$

S-4: $R=B n$<smiles>COC(=O)C(=O)Oc1cccc(OC(C)C(=O)O)c1I</smiles>

S-5

Table S2. Catalyst optimization. Unless otherwise noted, reactions were performed on $0.10 \mathrm{mmol}$ scale, with yields of 1,2-difluoride determined by ${ }^{1} \mathrm{H}$ NMR using nitrobenzene as an internal standard. ${ }^{a}$ Reaction was conducted on $1.00 \mathrm{mmol}$ scale, and the isolated yield of $\mathbf{3}$ is listed. ${ }^{b} \mathrm{~A}$ higher product ratio $(\mathbf{3 f : 4 f}=39: 1.0 \mathrm{vs.}$ 17:1.0) was achieved with catalyst $\mathbf{1 b}$ than with catalyst $\mathbf{1 a}$. 


\section{Preparation and Characterization of Catalysts and Substrates}

General Procedure A: To a stirred solution of carboxylic acid (1 equiv) in dichloromethane $(0.1 \mathrm{M})$ was added 1-hydroxybenzotriazole hydrate (1.5 equiv) and $N$-(3-dimethylaminopropyl)- $N$ '-ethylcarbodiimide hydrochloride (1.7 equiv). $N, N$-Diisopropylethylamine (4.0 equiv) and amine coupling partner (3.0 equiv) were added sequentially by syringe. After stirring for $12-18 \mathrm{~h}$, the solution was diluted with dichloromethane (10 $\mathrm{mL}$ per $1.00 \mathrm{mmol}$ of substrate) and washed with $1.0 \mathrm{M}$ aqueous $\mathrm{HCl}(10 \mathrm{~mL}$ per $1.00 \mathrm{mmol}$ of substrate), then with brine $\left(10 \mathrm{~mL}\right.$ per $1.00 \mathrm{mmol}$ of substrate). The organic layer was dried over anhydrous $\mathrm{MgSO}_{4}$, filtered, and concentrated under reduced pressure. The residue was purified by flash column chromatography on silica gel.

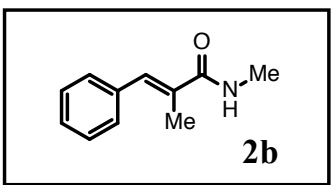

Prepared according to General Procedure A using $\alpha$-methylcinnamic acid (1.00 g, 6.17 mmol) and methylamine (2.0 M in THF, $9.25 \mathrm{~mL}, 18.5 \mathrm{mmol})$. After workup, the crude residue was purified by silica gel chromatography $(50 \%$ ethyl acetate in hexanes) to give $\mathbf{2 b}$ ( $959 \mathrm{mg}, 89 \%$ ) as a white solid.

${ }^{1} \mathrm{H}$ NMR $\left(600 \mathrm{MHz}, \mathrm{CDCl}_{3}\right) \delta 7.40-7.36(\mathrm{~m}, 2 \mathrm{H}), 7.35-7.32(\mathrm{~m}, 3 \mathrm{H}), 7.31-7.27$ $(\mathrm{m}, 1 \mathrm{H}), 5.88(\mathrm{br} \mathrm{s}, 1 \mathrm{H}), 2.95(\mathrm{~d}, J=4.9 \mathrm{~Hz}, 3 \mathrm{H}), 2.10(\mathrm{~d}, J=1.4 \mathrm{~Hz}, 3 \mathrm{H}) ;{ }^{13} \mathrm{C}$ NMR $\left(125.7 \mathrm{MHz}, \mathrm{CDCl}_{3}\right) \delta$ 170.4, 136.3, 133.8, 132.1, 129.4, 128.5, 127.9, 26.9, 14.4; FTIR (thin film) v 3311, 2937, 1649, 1611, 1531, $702 \mathrm{~cm}^{-1}$; HRMS (ESI-TOF) Calc'd for $\mathrm{C}_{11} \mathrm{H}_{14} \mathrm{NO}[\mathrm{M}+\mathrm{H}]^{+}:$176.1070; found 176.1068.

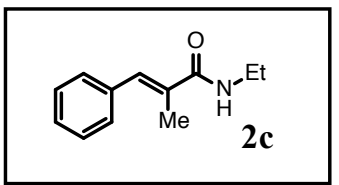

Prepared according to General Procedure A using $\alpha$-methylcinnamic acid (1.00 g, 6.17 $\mathrm{mmol})$ and ethylamine $(2.0 \mathrm{M}$ in THF, $9.25 \mathrm{~mL}, 18.5 \mathrm{mmol})$. After workup, the crude residue was purified by silica gel chromatography (20 to $50 \%$ ethyl acetate in hexanes) to give $2 \mathrm{c}(1.006 \mathrm{~g}, 86 \%)$ as a white solid.

${ }^{1} \mathrm{H}$ NMR $\left(500 \mathrm{MHz}, \mathrm{CDCl}_{3}\right) \delta 7.40-7.35(\mathrm{~m}, 2 \mathrm{H}), 7.35-7.27(\mathrm{~m}, 4 \mathrm{H}), 5.84$ (br s, $1 \mathrm{H}), 3.43(\mathrm{qd}, J=7.3,5.6 \mathrm{~Hz}, 2 \mathrm{H}), 2.10(\mathrm{~d}, J=1.4 \mathrm{~Hz}, 3 \mathrm{H}), 1.23(\mathrm{t}, J=7.3 \mathrm{~Hz}, 3 \mathrm{H}) ;{ }^{13} \mathrm{C} \mathrm{NMR}(125.7 \mathrm{MHz}$, $\left.\mathrm{CDCl}_{3}\right) \delta 169.6,136.4,133.7,132.3,129.4,128.5,127.9,35.0,15.1,14.4$; FTIR (thin film) $v 3291,2968$, 2943, 2876, 1641, 1617, 1532, $705 \mathrm{~cm}^{-1}$; HRMS (ESI-TOF) Calc'd for $\mathrm{C}_{12} \mathrm{H}_{16} \mathrm{NO}[\mathrm{M}+\mathrm{H}]^{+}:$190.1226; found 190.1225 .

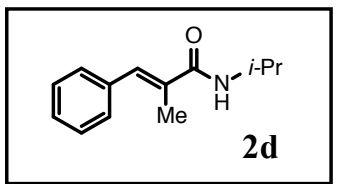

Prepared according to General Procedure A using $\alpha$-methylcinnamic acid (1.00 g, 6.17 $\mathrm{mmol})$ and isopropylamine $(1.51 \mathrm{~mL}, 18.5 \mathrm{mmol})$. After workup, the crude residue was purified by silica gel chromatography (30\% ethyl acetate in hexanes) to give $\mathbf{2 d}$ $(1.054 \mathrm{~g}, 84 \%)$ as a white solid.

${ }^{1} \mathrm{H}$ NMR $\left(500 \mathrm{MHz}, \mathrm{CDCl}_{3}\right) \delta 7.40-7.35(\mathrm{~m}, 2 \mathrm{H}), 7.35-7.27(\mathrm{~m}, 4 \mathrm{H}), 5.67$ (br s, $1 \mathrm{H}), 4.20(\mathrm{~m}, 1 \mathrm{H}), 2.09(\mathrm{~d}, J=1.4 \mathrm{~Hz}, 3 \mathrm{H}), 1.24(\mathrm{~d}, J=6.5 \mathrm{~Hz}, 6 \mathrm{H}) ;{ }^{13} \mathrm{C}$ NMR $\left(125.7 \mathrm{MHz}, \mathrm{CDCl}_{3}\right) \delta 169.0$, 136.3, 133.7, 132.5, 129.4, 128.5, 127.9, 41.9, 23.0, 14.4; FTIR (thin film) v 3295, 2975, 1638, 1614, 1530, $1358,706 \mathrm{~cm}^{-1}$; HRMS (ESI-TOF) Calc'd for $\mathrm{C}_{13} \mathrm{H}_{18} \mathrm{NO}[\mathrm{M}+\mathrm{H}]^{+}: 204.1383$; found 204.1382.

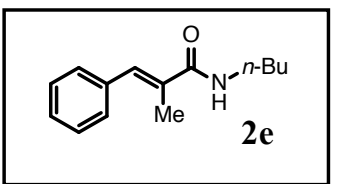

Prepared according to General Procedure A using $\alpha$-methylcinnamic acid (1.00 g, 6.17 mmol) and $n$-butylamine $(1.83 \mathrm{~mL}, 18.5 \mathrm{mmol})$. After workup, the crude residue was purified by silica gel chromatography (30\% ethyl acetate in hexanes) to give $2 \mathrm{e}$ (1.269 $\mathrm{g}, 95 \%)$ as a white solid.

${ }^{1} \mathrm{H}$ NMR $\left(500 \mathrm{MHz}, \mathrm{CDCl}_{3}\right) \delta \delta 7.40-7.35(\mathrm{~m}, 2 \mathrm{H}), 7.33(\mathrm{dd}, J=6.7,1.5 \mathrm{~Hz}, 3 \mathrm{H})$, $7.31-7.27(\mathrm{~m}, 1 \mathrm{H}), 5.85(\mathrm{br} \mathrm{s}, 1 \mathrm{H}), 3.39(\mathrm{td}, J=7.2,5.7 \mathrm{~Hz}, 2 \mathrm{H}), 2.10(\mathrm{~d}, J=1.4 \mathrm{~Hz}, 3 \mathrm{H}), 1.63-1.51(\mathrm{~m}$, $4 \mathrm{H}), 1.41(\mathrm{dq}, J=14.6,7.4 \mathrm{~Hz}, 2 \mathrm{H}), 0.96(\mathrm{t}, J=7.4 \mathrm{~Hz}, 3 \mathrm{H}) ;{ }^{13} \mathrm{C}$ NMR $\left(125.7 \mathrm{MHz}, \mathrm{CDCl}_{3}\right) \delta 169.7,136.4$, 133.7, 132.4, 129.4, 128.5, 127.9, 39.9, 31.9, 20.3, 14.5, 13.9; FTIR (thin film) v 3296, 2959, 2934, 2872, $1638,1613,1527,706 \mathrm{~cm}^{-1}$; HRMS (ESI-TOF) Calc'd for $\mathrm{C}_{14} \mathrm{H}_{20} \mathrm{NO}[\mathrm{M}+\mathrm{H}]^{+}: 218.1539$; found 218.1539 . 


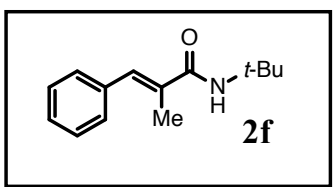

Prepared according to General Procedure A using $\alpha$-methylcinnamic acid (3.00 g, 18.5 mmol) and tert-butylamine $(5.83 \mathrm{~mL}, 55.5 \mathrm{mmol})$. After workup, the crude residue was purified by silica gel chromatography ( $20 \%$ ethyl acetate in hexanes) to give $\mathbf{2 f}$ $(3.538 \mathrm{~g}, 88 \%)$ as a white solid.

${ }^{1} \mathrm{H}$ NMR (500 MHz, $\left.\mathrm{CDCl}_{3}\right) \delta 7.40-7.34(\mathrm{~m}, 2 \mathrm{H}), 7.33-7.27(\mathrm{~m}, 3 \mathrm{H}), 7.25(\mathrm{~s}, 1 \mathrm{H})$, $5.70($ br s, $1 \mathrm{H}), 2.06(\mathrm{~d}, J=1.4 \mathrm{~Hz}, 3 \mathrm{H}), 1.44(\mathrm{~s}, 9 \mathrm{H}){ }^{13} \mathrm{C} \mathrm{NMR}\left(125.7 \mathrm{MHz}, \mathrm{CDCl}_{3}\right) \delta 169.2,136.5,133.6$, 133.0, 129.4, 128.4, 127.8, 51.5, 29.0, 14.5; FTIR (thin film) v 3315, 2967, 1647, 1619, 1527, 1450, $702 \mathrm{~cm}^{-1}$; HRMS (ESI-TOF) Calc'd for $\mathrm{C}_{14} \mathrm{H}_{20} \mathrm{NO}[\mathrm{M}+\mathrm{H}]^{+}: 218.1539$; found 218.1539.

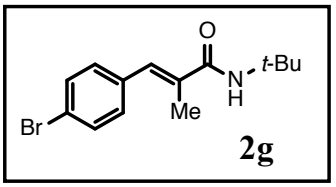

To a stirred solution of ethyl (E)-3-(4-bromophenyl)-2-methylacrylate ${ }^{1}$ (1.275 g, 4.74 mmol) in THF $(13.5 \mathrm{~mL})$ and ethanol $(13.5 \mathrm{~mL})$ was added $1.0 \mathrm{M}$ aqueous $\mathrm{NaOH}$ $(13.5 \mathrm{~mL})$. After $21 \mathrm{~h}, 1.0 \mathrm{M}$ aqueous $\mathrm{HCl}(20 \mathrm{~mL})$ and ethyl acetate $(40 \mathrm{~mL})$ were sequentially added. The organic layer was washed with brine $(20 \mathrm{~mL})$, dried over anhydrous $\mathrm{MgSO}_{4}$, and concentrated under reduced pressure to afford (E)-3-(4bromophenyl)-2-methylacrylic acid, which was used without further purification. The title compound was prepared according to General Procedure A using (E)-3-(4-bromophenyl)-2-methylacrylic acid (1.062 g, 4.41 $\mathrm{mmol})$ and tert-butylamine $(1.39 \mathrm{~mL}, 13.2 \mathrm{mmol})$. After workup, the crude residue was purified by silica gel chromatography (30\% ethyl acetate in hexanes) to give $2 \mathrm{~g}(1.087 \mathrm{~g}, 83 \%)$ as a white solid.

${ }^{1} \mathrm{H}$ NMR $\left(500 \mathrm{MHz}, \mathrm{CDCl}_{3}\right) \delta 7.53-7.47(\mathrm{~m}, 2 \mathrm{H}), 7.20-7.16(\mathrm{~m}, 3 \mathrm{H}), 5.68(\mathrm{~s}, 1 \mathrm{H}), 2.03(\mathrm{~d}, J=1.4 \mathrm{~Hz}, 3 \mathrm{H})$, $1.43(\mathrm{~s}, 9 \mathrm{H}) ;{ }^{13} \mathrm{C} \mathrm{NMR}\left(125.7 \mathrm{MHz}, \mathrm{CDCl}_{3}\right) \delta 168.9,135.4,134.2,131.9,131.6,130.9,121.8,51.6,28.9,14.5$; FTIR (thin film) $v 3323,2968,1649,1622,1527,1487,1221 \mathrm{~cm}^{-1}$; HRMS (ESI-TOF) Calc'd for $\mathrm{C}_{14} \mathrm{H}_{19} \mathrm{BrNO}$ $[\mathrm{M}+\mathrm{H}]^{+}: 296.0645$; found 296.0643 .

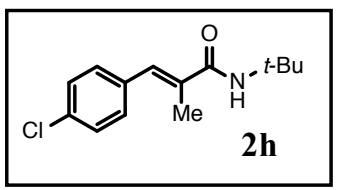

Prepared according to General Procedure A using (E)-3-(4-chlorophenyl)-2methylacrylic $\operatorname{acid}^{2}(785 \mathrm{mg}, 3.99 \mathrm{mmol})$ and tert-butylamine $(1.26 \mathrm{~mL}, 12.0 \mathrm{mmol})$. After workup, the crude residue was purified by silica gel chromatography (10 to $30 \%$ diethyl ether in hexanes) to give $\mathbf{2 h}(739 \mathrm{mg}, 74 \%)$ as a white solid.

${ }^{1} \mathrm{H}$ NMR $\left(500 \mathrm{MHz}, \mathrm{CDCl}_{3}\right) \delta 7.34(\mathrm{~d}, J=8.5 \mathrm{~Hz}, 2 \mathrm{H}), 7.24(\mathrm{~d}, J=8.5 \mathrm{~Hz}, 2 \mathrm{H}), 7.20$ (s, 1H), 5.68 (br s, 1H), $2.04(\mathrm{~d}, J=1.4 \mathrm{~Hz}, 3 \mathrm{H}), 1.43(\mathrm{~s}, 9 \mathrm{H}) ;{ }^{13} \mathrm{C}$ NMR $\left(125.7 \mathrm{MHz}, \mathrm{CDCl}_{3}\right.$ ) $\delta 168.9,134.9$, 134.1, 133.6, 131.8, 130.6, 128.7, 51.6, 28.9, 14.5; FTIR (thin film) v 3314, 2968, 2922, 1648, 1621, 1528, 1452, $1091 \mathrm{~cm}^{-1}$; HRMS (ESI-TOF) Calc'd for $\mathrm{C}_{14} \mathrm{H}_{19} \mathrm{ClNO}[\mathrm{M}+\mathrm{H}]^{+}: 252.1150$; found 252.1146.

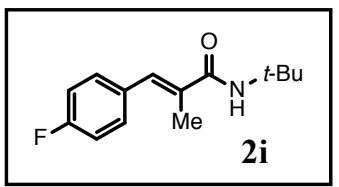

Prepared according to General Procedure A using (E)-3-(4-fluorophenyl)-2methylacrylic acid $(300 \mathrm{mg}, 1.67 \mathrm{mmol})$ and tert-butylamine $(0.53 \mathrm{~mL}, 5.01 \mathrm{mmol})$. After workup, the crude residue was purified by silica gel chromatography (35\% diethyl ether in hexanes) to give $\mathbf{2} \mathbf{i}(350 \mathrm{mg}, 89 \%)$ as a white solid.

${ }^{1} \mathrm{H}$ NMR $\left(500 \mathrm{MHz}, \mathrm{CDCl}_{3}\right) \delta 7.32-7.27(\mathrm{~m}, 2 \mathrm{H}), 7.22(\mathrm{~s}, 1 \mathrm{H}), 7.09-7.03(\mathrm{~m}, 2 \mathrm{H})$, $5.68(\mathrm{~s}, 1 \mathrm{H}), 2.04(\mathrm{~d}, J=1.5 \mathrm{~Hz}, 3 \mathrm{H}), 1.43(\mathrm{~s}, 9 \mathrm{H}) ;{ }^{13} \mathrm{C} \mathrm{NMR}\left(125.7 \mathrm{MHz}, \mathrm{CDCl}_{3}\right) \delta 169.0,162.2(\mathrm{~d}, J=247.9$ $\mathrm{Hz}), 133.4,132.5(\mathrm{~d}, J=3.6 \mathrm{~Hz}), 132.0,131.1(\mathrm{~d}, J=8.1 \mathrm{~Hz}), 115.5(\mathrm{~d}, J=21.5 \mathrm{~Hz}), 51.5,29.0,14.5 ;{ }^{19} \mathrm{~F}$ $\operatorname{NMR}\left(470.4 \mathrm{MHz}, \mathrm{CDCl}_{3}\right.$ ) $\delta-113.69$ (tt, $J=8.6,5.4 \mathrm{~Hz}$ ); FTIR (thin film) v 3317, 2968, 2926, 1651, 1622, 1508, $1226 \mathrm{~cm}^{-1}$; HRMS (ESI-TOF) Calc'd for $\mathrm{C}_{14} \mathrm{H}_{19} \mathrm{FNO}[\mathrm{M}+\mathrm{H}]^{+}: 236.1445$; found 236.1443.

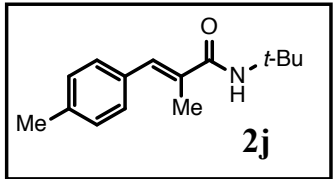

Prepared according to General Procedure A using (E)-2-methyl-3-( $p$-tolyl)acrylic $\operatorname{acid}^{2}(500 \mathrm{mg}, 2.84 \mathrm{mmol})$ and tert-butylamine $(0.90 \mathrm{~mL}, 8.52 \mathrm{mmol})$. After workup, the crude residue was purified by silica gel chromatography (0 to $20 \%$ diethyl ether in hexanes) to give $\mathbf{2} \mathbf{j}(532 \mathrm{mg}, 81 \%)$ as a white solid.

${ }^{1} \mathrm{H}$ NMR $\left(500 \mathrm{MHz}, \mathrm{CDCl}_{3}\right) \delta 7.22(\mathrm{~m}, J=8.1 \mathrm{~Hz}, 3 \mathrm{H}), 7.20-7.15(\mathrm{~m}, 2 \mathrm{H}), 5.68(\mathrm{~s}$, $1 \mathrm{H}), 2.36(\mathrm{~s}, 3 \mathrm{H}), 2.06(\mathrm{~d}, J=1.4 \mathrm{~Hz}, 3 \mathrm{H}), 1.43(\mathrm{~s}, 9 \mathrm{H}) ;{ }^{13} \mathrm{C} \mathrm{NMR}\left(125.7 \mathrm{MHz}, \mathrm{CDCl}_{3}\right) \delta 169.4,137.7,133.6$, 133.0, 132.8, 129.4, 129.2, 51.5, 29.0, 21.4, 14.5; FTIR (thin film) v 3298, 2972, 2951, 2918, 1646, 1619, 
1527, 1512, 1451, 1224, $812 \mathrm{~cm}^{-1}$; HRMS (ESI-TOF) Calc'd for $\mathrm{C}_{15} \mathrm{H}_{22} \mathrm{NO}[\mathrm{M}+\mathrm{H}]^{+}:$232.1696; found 232.1694

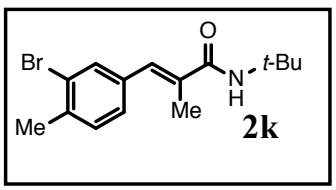

Triethyl 2-phosphonopropionate ( $1.63 \mathrm{~mL}, 7.54 \mathrm{mmol}, 1.5$ equiv) was slowly added to a dispersion of sodium hydride ( $349 \mathrm{mg}, 8.53 \mathrm{mmol}, 1.7$ equiv) in THF $(7.50 \mathrm{~mL})$ at 0 ${ }^{\circ} \mathrm{C}$. The resulting mixture was stirred for $1 \mathrm{~h}$. The mixture was then cooled to $-78{ }^{\circ} \mathrm{C}$, and 3-bromo-4-methylbenzaldehyde ( $1.00 \mathrm{~g}, 5.02 \mathrm{mmol}, 1.0$ equiv) was slowly added. After stirring at room temperature for $15 \mathrm{~h}$, the mixture was poured over saturated aqueous ammonium chloride, and the phases were separated. The aqueous phase was extracted with diethyl ether, and the combined organic layers were washed with brine, dried over anhydrous magnesium sulfate, and concentrated under reduced pressure. The crude residue was purified by silica gel chromatography (0 to $10 \%$ ethyl acetate in hexanes). The resulting colorless oil $(1.275 \mathrm{~g}$, $4.74 \mathrm{mmol})$ was dissolved in THF $(13.5 \mathrm{~mL})$ and ethanol $(13.5 \mathrm{~mL})$ To the stirred solution was added $1.0 \mathrm{M}$ aqueous $\mathrm{NaOH}(13.5 \mathrm{~mL})$. After $21 \mathrm{~h}, 1.0 \mathrm{M}$ aqueous $\mathrm{HCl}(20 \mathrm{~mL})$ and ethyl acetate $(40 \mathrm{~mL})$ were sequentially added. The organic layer was washed with brine $(20 \mathrm{~mL})$, dried over anhydrous $\mathrm{MgSO}_{4}$, and concentrated under reduced pressure to afford (E)-3-(3bromo-4-methylphenyl)-2-methylacrylic acid, which was used without further purification. The spectral data for this compound were in accordance with the literature. ${ }^{3}$ The title compound was prepared according to General Procedure A using (E)-3-(3-bromo-4-methylphenyl)-2-methylacrylic acid (896 mg, $3.51 \mathrm{mmol}$ ) and tert-butylamine $(1.10 \mathrm{~mL}, 10.5 \mathrm{mmol})$. After workup, the crude residue was purified by silica gel chromatography ( $25 \%$ ethyl acetate in hexanes) to give $2 \mathbf{k}(979.4 \mathrm{mg}, 90 \%)$ as a white solid.

${ }^{1} \mathrm{H}$ NMR $\left(500 \mathrm{MHz}, \mathrm{CDCl}_{3}\right) \delta 7.49(\mathrm{~d}, J=1.6 \mathrm{~Hz}, 1 \mathrm{H}), 7.24-7.21(\mathrm{~m}, 1 \mathrm{H}), 7.18-7.13(\mathrm{~m}, 2 \mathrm{H}), 2.40(\mathrm{~s}, 3 \mathrm{H})$, $2.05(\mathrm{~d}, J=1.4 \mathrm{~Hz}, 3 \mathrm{H}), 1.43(\mathrm{~s}, 9 \mathrm{H}) ;{ }^{13} \mathrm{C}$ NMR $\left(125.7 \mathrm{MHz}, \mathrm{CDCl}_{3}\right) \delta 168.9,137.5,135.9,134.2,133.0$, 131.4, 130.7, 128.3, 124.9, 51.6, 29.0, 22.9, 14.5; FTIR (thin film) v 3308, 2967, 2922, 1649, 1619, 1527, 1221 $\mathrm{cm}^{-1}$; HRMS (ESI-TOF) Calc'd for $\mathrm{C}_{15} \mathrm{H}_{21} \mathrm{BrNO}[\mathrm{M}+\mathrm{H}]^{+}: 310.0801$; found 310.0800 .

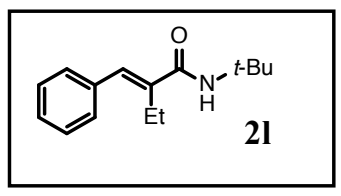

Prepared according to General Procedure A using (E)-2-benzylidenebutanoic acid ${ }^{4}$ $(723 \mathrm{mg}, 4.10 \mathrm{mmol})$ and tert-butylamine $(1.29 \mathrm{~mL}, 12.3 \mathrm{mmol})$. After workup, the crude residue was purified by silica gel chromatography $(10 \%$ ethyl acetate in hexanes) to give 21 ( $816.6 \mathrm{mg}, 86 \%)$ as a white solid.

${ }^{1} \mathrm{H} \mathrm{NMR}\left(500 \mathrm{MHz}, \mathrm{CDCl}_{3}\right) \delta 7.39-7.34(\mathrm{~m}, 2 \mathrm{H}), 7.32-7.27(\mathrm{~m}, 3 \mathrm{H}), 7.01(\mathrm{~s}, 1 \mathrm{H})$, $5.70(\mathrm{br} \mathrm{s}, 1 \mathrm{H}), 2.51$ (q, $J=7.5 \mathrm{~Hz}, 2 \mathrm{H}), 1.44(\mathrm{~s}, 9 \mathrm{H}), 1.13(\mathrm{t}, J=7.5 \mathrm{~Hz}, 3 \mathrm{H}) ;{ }^{13} \mathrm{C} \mathrm{NMR}\left(125.7 \mathrm{MHz}, \mathrm{CDCl}_{3}\right)$ $\delta 169.5,141.6,136.4,131.0,128.9,128.5,127.7,51.5,29.0,21.4,13.6$; FTIR (thin film) v 3310, 2965, 1642, $1619,1536,700 \mathrm{~cm}^{-1}$; HRMS (ESI-TOF) Calc'd for $\mathrm{C}_{15} \mathrm{H}_{22} \mathrm{NO}[\mathrm{M}+\mathrm{H}]^{+}: 232.1696$; found 232.1695.

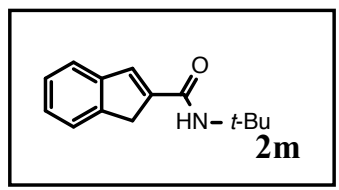

Prepared according to General Procedure A using $1 H$-indene-2-carboxylic acid (200 $\mathrm{mg}, 1.25 \mathrm{mmol})$ and tert-butylamine $(0.39 \mathrm{~mL}, 3.75 \mathrm{mmol})$. After workup, the crude residue was purified by silica gel chromatography (10\% ethyl acetate in hexanes) to give $\mathbf{2 m}(212 \mathrm{mg}, 79 \%)$ as a white solid.

${ }^{1} \mathrm{H}$ NMR $\left(500 \mathrm{MHz}, \mathrm{CDCl}_{3}\right) \delta 7.51-7.44(\mathrm{~m}, 2 \mathrm{H}), 7.36(\mathrm{td}, J=1.8,0.7 \mathrm{~Hz}, 1 \mathrm{H}), 7.33$ $-7.27(\mathrm{~m}, 2 \mathrm{H}), 5.73(\mathrm{br} \mathrm{s}, 1 \mathrm{H}), 3.65-3.63(\mathrm{~m}, 2 \mathrm{H}), 1.46(\mathrm{~s}, 9 \mathrm{H}) ;{ }^{13} \mathrm{C} \mathrm{NMR}\left(125.7 \mathrm{MHz}, \mathrm{CDCl}_{3}\right) \delta 164.5$, 143.9, 143.3, 142.6, 134.9, 127.0, 126.9, 124.6, 122.8, 51.6, 38.4, 29.1; FTIR (thin film) v 3246, 3069, 3055, 2980, 2960, 2929, 1628, 1588, 1563, 1532, 1270, 1218, 758, $713 \mathrm{~cm}^{-1}$; HRMS (ESI-TOF) Calc'd for $\mathrm{C}_{14} \mathrm{H}_{18} \mathrm{NO}[\mathrm{M}+\mathrm{H}]^{+}: 216.1383$; found 216.1380 .

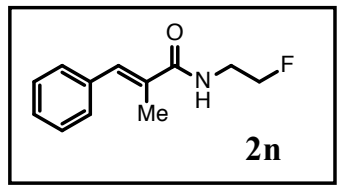

Prepared according to General Procedure A using $\alpha$-methylcinnamic acid (1.00 g, $6.17 \mathrm{mmol})$ and 2-fluoroethylamine hydrochloride $(1.84 \mathrm{~g}, 18.5 \mathrm{mmol})$. After workup, the crude residue was purified by silica gel chromatography $(50 \%$ diethyl ether in hexanes) to give $\mathbf{2 n}(1.037 \mathrm{~g}, 81 \%)$ as a white solid.

${ }^{1} \mathrm{H}$ NMR (500 MHz, $\left.\mathrm{CDCl}_{3}\right) \delta 7.42-7.37(\mathrm{~m}, 3 \mathrm{H}), 7.36-7.28(\mathrm{~m}, 3 \mathrm{H}), 6.24$ (br s, 
$1 \mathrm{H}), 4.59(\mathrm{dt}, J=47.5,4.7 \mathrm{~Hz}, 2 \mathrm{H}), 3.72(\mathrm{ddt}, J=28.5,5.7,4.6 \mathrm{~Hz}, 2 \mathrm{H}), 2.12(\mathrm{~d}, J=1.4 \mathrm{~Hz}, 3 \mathrm{H}) ;{ }^{13} \mathrm{C} \mathrm{NMR}$ $\left(125.7 \mathrm{MHz}, \mathrm{CDCl}_{3}\right) \delta 169.8,136.1,134.6,131.7,129.5,128.5,128.1,83.0(\mathrm{~d}, J=166.2 \mathrm{~Hz}), 40.5(\mathrm{~d}, J=19.5$ $\mathrm{Hz}$ ), 14.4; ${ }^{19} \mathrm{~F}$ NMR $\left(470.4 \mathrm{MHz}, \mathrm{CDCl}_{3}\right.$ ) $\delta-224.2$ (tt, $J=47.3,28.4 \mathrm{~Hz}, 1 \mathrm{~F}$ ); FTIR (thin film) v 3279, 3052, 2960, 1643, 1619, 1531, $705 \mathrm{~cm}^{-1}$; HRMS (ESI-TOF) Calc'd for $\mathrm{C}_{12} \mathrm{H}_{15} \mathrm{FNO}[\mathrm{M}+\mathrm{H}]^{+}:$208.1132; found 208.1129 .

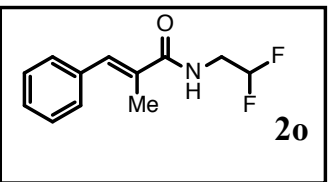

Prepared according to General Procedure A using $\alpha$-methylcinnamic acid (1.00 g, $6.17 \mathrm{mmol}$ ) and with the following modification: 2.0 equiv of 2,2-difluoroethan-1amine $(0.87 \mathrm{~mL}, 12.3 \mathrm{mmol})$ were used. After workup, the crude residue was purified by silica gel chromatography ( 10 to $50 \%$ diethyl ether in hexanes) to give 20 (1.094 $\mathrm{g}, 79 \%)$ as a white solid.

${ }^{1} \mathrm{H}$ NMR $\left(500 \mathrm{MHz}, \mathrm{CDCl}_{3}\right) \delta 7.42-7.37(\mathrm{~m}, 3 \mathrm{H}), 7.36-7.30(\mathrm{~m}, 3 \mathrm{H}), 6.10$ (br s, 1H), $5.94(\mathrm{tt}, J=56.3,4.2$ $\mathrm{Hz}, 1 \mathrm{H}), 3.77$ (tdd, $J=14.8,6.2,4.2 \mathrm{~Hz}, 2 \mathrm{H}), 2.13(\mathrm{~d}, J=1.4 \mathrm{~Hz}, 3 \mathrm{H}) ;{ }^{13} \mathrm{C}$ NMR $\left(125.7 \mathrm{MHz}, \mathrm{CDCl}_{3}\right) \delta$ 170.0, 135.9, 135.2, 131.2, 129.5, 128.6, $128.3,113.8(\mathrm{t}, J=241.3 \mathrm{~Hz}), 42.4(\mathrm{t}, J=26.5 \mathrm{~Hz}), 14.4 ;{ }^{19} \mathrm{~F}$ NMR $\left(470.4 \mathrm{MHz}, \mathrm{CDCl}_{3}\right) \delta-122.9(\mathrm{dt}, J=56.4,14.8 \mathrm{~Hz}, 2 \mathrm{~F})$; FTIR (thin film) $v 3290,1646,1616,1524,1113$, $1057,695 \mathrm{~cm}^{-1}$; HRMS (ESI-TOF) Calc'd for $\mathrm{C}_{12} \mathrm{H}_{14} \mathrm{~F}_{2} \mathrm{NO}[\mathrm{M}+\mathrm{H}]^{+}: 226.1038$; found 226.1035 .

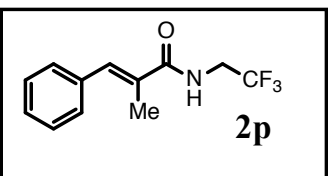

Prepared according to General Procedure A using $\alpha$-methylcinnamic acid (1.00 g, $6.17 \mathrm{mmol})$ and 2,2,2-trifluoroethan-1-amine $(1.45 \mathrm{~mL}, 18.5 \mathrm{mmol})$. After workup, the crude residue was purified by silica gel chromatography (20 to $50 \%$ ethyl acetate in hexanes) to give $2 \mathrm{p}(1.365 \mathrm{~g}, 91 \%)$ as a white solid.

${ }^{1} \mathrm{H}$ NMR $\left(500 \mathrm{MHz}, \mathrm{CDCl}_{3}\right) \delta 7.44-7.37(\mathrm{~m}, 3 \mathrm{H}), 7.37-7.30(\mathrm{~m}, 3 \mathrm{H}), 6.10(\mathrm{br} \mathrm{s}$, $1 \mathrm{H}), 4.07(\mathrm{qd}, J=9.1,6.4 \mathrm{~Hz}, 2 \mathrm{H}), 2.14(\mathrm{~d}, J=1.4 \mathrm{~Hz}, 3 \mathrm{H}) ;{ }^{13} \mathrm{C}$ NMR $\left(125.7 \mathrm{MHz}, \mathrm{CDCl}_{3}\right) \delta 169.6,135.7$, 135.5, 131.1, 129.5, 128.6, 128.4, $124.4(\mathrm{q}, J=278.4 \mathrm{~Hz}), 41.2(\mathrm{q}, J=34.6 \mathrm{~Hz}), 14.4 ;{ }^{19} \mathrm{~F}$ NMR $(470.4 \mathrm{MHz}$, $\left.\mathrm{CDCl}_{3}\right) \delta-72.4\left(\mathrm{t}, J=9.2 \mathrm{~Hz}, 3 \mathrm{~F}\right.$ ); FTIR (thin film) $v 3277,1649,1625,1532,1258,1153 \mathrm{~cm}^{-1}$; HRMS (ESITOF) Calc'd for $\mathrm{C}_{12} \mathrm{H}_{13} \mathrm{~F}_{3} \mathrm{NO}[\mathrm{M}+\mathrm{H}]^{+}:$244.0944; found 244.0942.

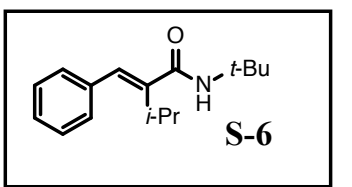

Prepared according to General Procedure A using (E)-2-benzylidene-3methylbutanoic acid $^{5}(187 \mathrm{mg}, 0.98 \mathrm{mmol})$ and tert-butylamine $(0.31 \mathrm{~mL}, 2.94$ mmol). After workup, the crude residue was purified by silica gel chromatography (20\% ethyl acetate in hexanes) to give $\mathbf{S - 6}(153 \mathrm{mg}, 63 \%)$ as a white solid.

${ }^{1} \mathrm{H}$ NMR $\left(500 \mathrm{MHz}, \mathrm{CDCl}_{3}\right) \delta 7.38-7.33(\mathrm{~m}, 2 \mathrm{H}), 7.29-7.25(\mathrm{~m}, 3 \mathrm{H}), 6.65(\mathrm{~s}, 1 \mathrm{H})$, $5.57(\mathrm{~s}, 1 \mathrm{H}), 3.08-2.97(\mathrm{~m}, 1 \mathrm{H}), 1.43(\mathrm{~s}, 9 \mathrm{H}), 1.23(\mathrm{~d}, J=6.9 \mathrm{~Hz}, 6 \mathrm{H}) ;{ }^{13} \mathrm{C} \mathrm{NMR}\left(125.7 \mathrm{MHz}, \mathrm{CDCl}_{3}\right) \delta$ 170.7, 147.0, 136.2, 128.8, 128.5, 128.3, 127.5, 51.6, 36.5, 29.0, 28.4, 21.7; FTIR (thin film) v 3312, 2973, 2922, 1640, 1617, 1532, $702 \mathrm{~cm}^{-1}$; HRMS (ESI-TOF) Calc'd for $\mathrm{C}_{16} \mathrm{H}_{24} \mathrm{NO}[\mathrm{M}+\mathrm{H}]^{+}:$246.1852; found 246.1850 . 


\section{Synthesis and Characterization of 1,2-Difluorides}

General Procedure B: A polyethylene conical tube equipped with a stir bar was charged with the alkene substrate (1 equiv), catalyst $\mathbf{1 b}(20.0 \mathrm{~mol} \%)$, and dichloromethane $(2.00 \mathrm{~mL}$ per $1.00 \mathrm{mmol}$ of substrate) at room temperature. The resulting mixture was cooled to $-78{ }^{\circ} \mathrm{C}$, and pyridinium poly(hydrogen fluoride) (pyr $\bullet \mathrm{HF}, 70 \%$ hydrogen fluoride by weight, 50 equiv hydrogen fluoride) was added via syringe, followed by $m$-chloroperbenzoic acid ( $m$ CPBA, $77 \%$ by weight, 1.30 equiv). The tube was sealed, and the mixture was warmed to $4{ }^{\circ} \mathrm{C}$ and stirred for $24 \mathrm{~h}$. The mixture was then cooled to $-78{ }^{\circ} \mathrm{C}$, diluted with dichloromethane (10.0 mL per $1.00 \mathrm{mmol}$ of substrate), and pipetted slowly into a suspension of basic alumina (10 g per 1.00 mmol of substrate) in dichloromethane $\left(50 \mathrm{~mL}\right.$ per $1.00 \mathrm{mmol}$ of substrate) at $-78{ }^{\circ} \mathrm{C}$. The resulting suspension was warmed to room temperature with stirring. The suspension was then filtered, and the filter cake was washed with dichloromethane $(200 \mathrm{~mL}$ per $1.00 \mathrm{mmol}$ of substrate). The combined filtrates were concentrated under reduced pressure. The crude product mixture was analyzed by ${ }^{19} \mathrm{~F}$ NMR to determine the ratio of $1,2-$ difluoride to 1,1-difluoride. The 1,1-difluoride products were assigned by analogy to previously reported 1,1difluorides. ${ }^{3}$ The crude product mixture was then purified by flash column chromatography on silica gel, and pure 1,2-difluoride was isolated and characterized.

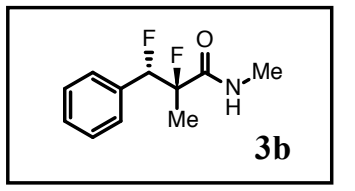

Prepared according to General Procedure B using 2b (175 mg, $1.00 \mathrm{mmol})$. Analysis of the crude product mixture by ${ }^{19} \mathrm{~F}$ NMR indicated a 1,2:1,1 ratio of 3.3:1.0. After workup, the crude residue was purified by silica gel chromatography (10 to $30 \%$ diethyl ether in hexanes) to give $\mathbf{3 b}(108 \mathrm{mg}, 51 \%)$ as a white solid. $\mathbf{3 b}$ was determined to be of $94 \%$ e.e. by chiral GC analysis $\left(\beta\right.$-Cyclosil, $40 \rightarrow 200{ }^{\circ} \mathrm{C}, 1 \% \mathrm{~min}$, 7 psi, $\mathrm{t}_{\mathrm{R}}($ major $)=94.910 \mathrm{~min}, \mathrm{t}_{\mathrm{R}}($ minor $\left.)=96.848 \mathrm{~min}\right)$.

${ }^{1} \mathrm{H}$ NMR $\left(500 \mathrm{MHz}, \mathrm{CDCl}_{3}\right) \delta 7.36-7.31(\mathrm{~m}, 5 \mathrm{H}), 5.99(\mathrm{br} \mathrm{s}, 1 \mathrm{H}), 5.72(\mathrm{dd}, J=44.1,25.7 \mathrm{~Hz}, 1 \mathrm{H}), 2.63(\mathrm{dd}, J$ $=5.1,0.6 \mathrm{~Hz}, 3 \mathrm{H}), 1.79(\mathrm{dd}, J=22.5,1.8 \mathrm{~Hz}, 3 \mathrm{H}) ;{ }^{13} \mathrm{C} \mathrm{NMR}\left(125.7 \mathrm{MHz}, \mathrm{CDCl}_{3}\right) \delta 170.0(\mathrm{dd}, J=19.4,7.3$ $\mathrm{Hz}), 135.8(\mathrm{dd}, J=23.0,1.3 \mathrm{~Hz}), 129.0,128.1,127.1(\mathrm{~d}, J=7.2 \mathrm{~Hz}), 98.5(\mathrm{dd}, J=196.1,23.0 \mathrm{~Hz}), 94.4(\mathrm{dd}, J$ $=184.2,19.4 \mathrm{~Hz}), 25.8,20.2(\mathrm{dd}, J=24.4,5.3 \mathrm{~Hz}) ;{ }^{19} \mathrm{~F}$ NMR $\left(470.4 \mathrm{MHz}, \mathrm{CDCl}_{3}\right) \delta-171.42--171.71(\mathrm{~m}$, 1F), -196.87 (dd, $J=44.2,9.7 \mathrm{~Hz}, 1 \mathrm{~F}$ ); FTIR (thin film) $\vee 3342,1658,1552,729 \mathrm{~cm}^{-1}$; HRMS (ESI-TOF) Calc'd for $\mathrm{C}_{11} \mathrm{H}_{14} \mathrm{~F}_{2} \mathrm{NO}[\mathrm{M}+\mathrm{H}]^{+}: 214.1038$; found 214.1036; $[\alpha]_{\mathrm{D}}^{22}=-37.7\left(\mathrm{c}=0.030, \mathrm{CHCl}_{3}\right)$.

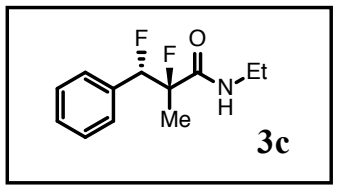

Prepared according to General Procedure B using 2c (189 mg, $1.00 \mathrm{mmol})$. Analysis of the crude product mixture by ${ }^{19} \mathrm{~F}$ NMR indicated a 1,2:1,1 ratio of 4.3:1.0. After workup, the crude residue was purified by silica gel chromatography (5 to $20 \%$ diethyl ether in hexanes) to give 3c (138 mg, 61\%) as a white solid. 3c was determined to be of $95 \%$ e.e. by chiral GC analysis ( $\beta$-Cyclosil, $40 \rightarrow 200{ }^{\circ} \mathrm{C}, 1.5$ $\%$ min, $14 \mathrm{psi}, \mathrm{t}_{\mathrm{R}}($ major $)=60.848 \mathrm{~min}, \mathrm{t}_{\mathrm{R}}($ minor $\left.)=61.554 \mathrm{~min}\right)$.

${ }^{1} \mathrm{H}$ NMR (500 MHz, $\left.\mathrm{CDCl}_{3}\right) \delta$ 7.34-7.33 (m, 5H), $5.96(\mathrm{br} \mathrm{s}, 1 \mathrm{H}), 5.71(\mathrm{dd}, J=44.2,25.7 \mathrm{~Hz}, 1 \mathrm{H}), 3.22-3.11$ $(\mathrm{m}, 1 \mathrm{H}), 3.11-3.00(\mathrm{~m}, 1 \mathrm{H}), 1.79(\mathrm{dd}, J=22.5,1.6 \mathrm{~Hz}, 3 \mathrm{H}), 0.87(\mathrm{dd}, J=7.7,7.0 \mathrm{~Hz}, 3 \mathrm{H}) ;{ }^{13} \mathrm{C}$ NMR $(125.7$ $\left.\mathrm{MHz}, \mathrm{CDCl}_{3}\right) \delta 169.2(\mathrm{dd}, J=19.2,7.3 \mathrm{~Hz}), 134.2(\mathrm{~d}, J=21.8 \mathrm{~Hz}), 129.0,128.1,127.2(\mathrm{~d}, J=7.1 \mathrm{~Hz}), 98.2$ $(\mathrm{dd}, J=196.3,22.7 \mathrm{~Hz}), 94.4(\mathrm{dd}, J=184.6,19.2 \mathrm{~Hz}), 33.9,20.1(\mathrm{dd}, J=24.4,5.0 \mathrm{~Hz}), 14.5 ;{ }^{19} \mathrm{~F} \mathrm{NMR}(470.4$ $\mathrm{MHz}, \mathrm{CDCl}_{3}$ ) $\delta-171.60$ (dtdd, $\left.J=48.8,22.4,9.7,5.4 \mathrm{~Hz}, 1 \mathrm{~F}\right),-196.61$ (ddd, $J=44.1,10.0,1.8 \mathrm{~Hz}, 1 \mathrm{~F}$ ); FTIR (thin film) v 3336, 1651, 1550, 1109, $693 \mathrm{~cm}^{-1}$; HRMS (ESI-TOF) Calc'd for $\mathrm{C}_{12} \mathrm{H}_{16} \mathrm{~F}_{2} \mathrm{NO}[\mathrm{M}+\mathrm{H}]^{+}: 228.1194$; found 228.1192; ; $[\alpha]_{\mathrm{D}}{ }^{21}=-12.7\left(\mathrm{c}=0.069, \mathrm{CHCl}_{3}\right)$.

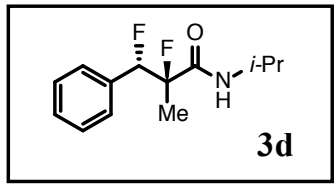

Prepared according to General Procedure B using 2d (203 mg, $1.00 \mathrm{mmol})$. Analysis of the crude product mixture by ${ }^{19} \mathrm{~F}$ NMR indicated a 1,2:1,1 ratio of 4.8:1.0. After workup, the crude residue was purified by silica gel chromatography (0 to $12 \%$ diethyl ether in hexanes) to give 3d (135 $\mathrm{mg}, 56 \%$ ) as a white solid. 3d was determined to be of $95 \%$ e.e. by chiral GC analysis ( $\beta$-Cyclosil, $40 \rightarrow 200{ }^{\circ} \mathrm{C}, 1.5$ $\%$ min, 14 psi, $\mathrm{t}_{\mathrm{R}}($ minor $)=59.224 \mathrm{~min}, \mathrm{t}_{\mathrm{R}}($ major $\left.)=59.726 \mathrm{~min}\right)$. 
${ }^{1} \mathrm{H}$ NMR $\left(500 \mathrm{MHz}, \mathrm{CDCl}_{3}\right) \delta$ 7.35-7.33 (m, 5H), $5.69(\mathrm{~m}, 2 \mathrm{H}), 4.03-3.67(\mathrm{~m}, 1 \mathrm{H}), 1.79(\mathrm{dd}, J=22.5,1.7$ $\mathrm{Hz}, 3 \mathrm{H}), 1.04(\mathrm{~d}, J=6.6 \mathrm{~Hz}, 3 \mathrm{H}), 0.75(\mathrm{~d}, J=6.5 \mathrm{~Hz}, 3 \mathrm{H}) ;{ }^{13} \mathrm{C}$ NMR $\left(125.7 \mathrm{MHz}, \mathrm{CDCl}_{3}\right) \delta 168.3(\mathrm{dd}, J=$ 19.1, $7.1 \mathrm{~Hz}), 134.19$ (d, $J=21.8 \mathrm{~Hz}), 129.0,128.1,127.3$ (d, $J=7.3 \mathrm{~Hz}), 98.1(\mathrm{dd}, J=196.4,22.7 \mathrm{~Hz}), 94.5$ $(\mathrm{dd}, J=184.4,18.9 \mathrm{~Hz}), 41.1,22.4,22.2,20.1(\mathrm{dd}, J=24.5,4.9 \mathrm{~Hz}) ;{ }^{19} \mathrm{~F}$ NMR $\left(470.4 \mathrm{MHz}, \mathrm{CDCl}_{3}\right) \delta-171.43$ $--171.76(\mathrm{~m}, 1 \mathrm{~F}),-196.40(\mathrm{dd}, J=44.1,9.8 \mathrm{~Hz}, 1 \mathrm{~F})$; FTIR (thin film) $v 3333,1646,1544,720 \mathrm{~cm}^{-1}$; HRMS (ESI-TOF) Calc'd for $\mathrm{C}_{13} \mathrm{H}_{18} \mathrm{~F}_{2} \mathrm{NO}[\mathrm{M}+\mathrm{H}]^{+}: 242.1351$; found 242.1348; ; $\left.\alpha\right]_{\mathrm{D}}^{22}=-3.9\left(\mathrm{c}=0.058, \mathrm{CHCl}_{3}\right)$.

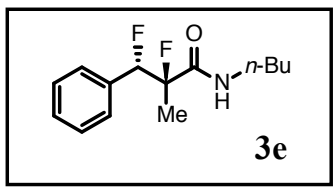

Prepared according to General Procedure B using 2e (217 mg, $1.00 \mathrm{mmol})$. Analysis of the crude product mixture by ${ }^{19} \mathrm{~F}$ NMR indicated a 1,2:1,1 ratio of 6.9:1.0. After workup, the crude residue was purified by silica gel chromatography (2 to $16 \%$ diethyl ether in hexanes) to give $\mathbf{3 e}(161 \mathrm{mg}, 63 \%)$ as a white solid. 3e was determined to be of $94 \%$ e.e. by chiral GC analysis (CP-Chirasil-Dex CB, $120{ }^{\circ} \mathrm{C}, 14$ psi, $\mathrm{t}_{\mathrm{R}}($ major $)=34.262 \mathrm{~min}, \mathrm{t}_{\mathrm{R}}($ minor $\left.)=35.790 \mathrm{~min}\right)$.

${ }^{1} \mathrm{H}$ NMR $\left(500 \mathrm{MHz}, \mathrm{CDCl}_{3}\right) \delta$ 7.35-7.33 (m, 5H), 5.97 (br s, 1H), $5.70(\mathrm{dd}, J=44.1,25.7 \mathrm{~Hz}, 1 \mathrm{H}), 3.19-3.09$ (m, 1H), $2.99(\mathrm{~m}, 1 \mathrm{H}), 1.79(\mathrm{dd}, J=22.5,1.7 \mathrm{~Hz}, 1 \mathrm{H}), 1.28-1.10(\mathrm{~m}, 2 \mathrm{H}), 1.10-0.94(\mathrm{~m}, 2 \mathrm{H}), 0.82-0.77$ $(\mathrm{m}, 3 \mathrm{H}) ;{ }^{13} \mathrm{C}$ NMR $\left(125.7 \mathrm{MHz}, \mathrm{CDCl}_{3}\right) \delta 169.2(\mathrm{dd}, J=19.0,7.2 \mathrm{~Hz}), 134.2(\mathrm{~d}, J=21.6 \mathrm{~Hz}), 129.0(\mathrm{~d}, J=$ $1.7 \mathrm{~Hz}$ ), 128.1, 127.3 (d, $J=7.0 \mathrm{~Hz}), 98.3(\mathrm{dd}, J=196.3,22.9 \mathrm{~Hz}), 94.5$ (dd, $J=184.0,19.0 \mathrm{~Hz}), 38.7,31.2$, $20.2(\mathrm{dd}, J=24.4,5.0 \mathrm{~Hz}), 19.7,13.7 ;{ }^{19} \mathrm{~F}$ NMR $\left(470.4 \mathrm{MHz}, \mathrm{CDCl}_{3}\right) \delta-171.50--171.73(\mathrm{~m}, 1 \mathrm{~F}),-196.19$ (ddd, $J=44.1,9.6,1.8 \mathrm{~Hz}, 1 \mathrm{~F}$ ); FTIR (thin film) $v 3341,2955,1652,1545 \mathrm{~cm}^{-1}$; HRMS (ESI-TOF) Calc'd for $\mathrm{C}_{14} \mathrm{H}_{20} \mathrm{~F}_{2} \mathrm{NO}[\mathrm{M}+\mathrm{H}]^{+}: 256.1507$; found 256.1506; $[\alpha]_{\mathrm{D}}{ }^{21}=-13.5\left(\mathrm{c}=0.11, \mathrm{CHCl}_{3}\right)$.

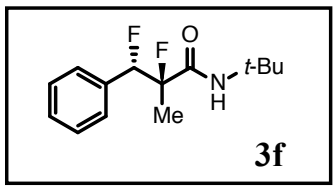

Prepared according to General Procedure B using 2f (217 mg, $1.00 \mathrm{mmol})$. Analysis of the crude product mixture by ${ }^{19} \mathrm{~F}$ NMR indicated a 1,2:1,1 ratio of 39:1.0. After workup, the crude residue was purified by silica gel chromatography (2 to $10 \%$ diethyl ether in hexanes) to give $\mathbf{3 f}(207 \mathrm{mg}, 81 \%)$ as a white solid. $3 \mathbf{f}$ was determined to be of $96 \%$ e.e. by chiral GC analysis $\left(\beta\right.$-Cyclosil, $40 \rightarrow 200{ }^{\circ} \mathrm{C}, 1.5 \% \mathrm{~min}, 14 \mathrm{psi}$, $\mathrm{t}_{\mathrm{R}}($ minor $)=57.689 \mathrm{~min}, \mathrm{t}_{\mathrm{R}}($ major $\left.)=58.359 \mathrm{~min}\right)$.

${ }^{1} \mathrm{H}$ NMR $\left(500 \mathrm{MHz}, \mathrm{CDCl}_{3}\right) \delta 7.38-7.32(\mathrm{~m}, 5 \mathrm{H}), 5.77(\mathrm{br} \mathrm{s}, 1 \mathrm{H}), 5.68(\mathrm{dd}, J=55,25 \mathrm{~Hz}, 1 \mathrm{H}), 1.77(\mathrm{dd}, J=$ $22.5,1.7 \mathrm{~Hz}, 3 \mathrm{H}), 1.14(\mathrm{~s}, 9 \mathrm{H}) ;{ }^{13} \mathrm{C}$ NMR $\left(125.7 \mathrm{MHz}, \mathrm{CDCl}_{3}\right) \delta 168.4(\mathrm{dd}, J=17.6,7.2 \mathrm{~Hz}), 134.2(\mathrm{~d}, J=$ $21.9 \mathrm{~Hz}), 129.0,128.1,127.5(\mathrm{~d}, J=6.8 \mathrm{~Hz}), 97.9$ (dd, $J=197.3,22.5 \mathrm{~Hz}), 94.6$ (dd, $J=184.0,19.0 \mathrm{~Hz}), 51.2$, 28.4, $20.2(\mathrm{dd}, J=24.6,5.0 \mathrm{~Hz}) ;{ }^{19} \mathrm{~F}$ NMR $\left(470.4 \mathrm{MHz}, \mathrm{CDCl}_{3}\right) \delta-169.28--169.57(\mathrm{~m}, 1 \mathrm{~F}),-196.25(\mathrm{dd}, J=$ 44.1, 9.7 Hz, 1F); FTIR (thin film) v 3443, 3387, 2970, 1682, 1528, 1022, $714 \mathrm{~cm}^{-1}$; HRMS (ESI-TOF) Calc'd for $\mathrm{C}_{14} \mathrm{H}_{20} \mathrm{~F}_{2} \mathrm{NO}[\mathrm{M}+\mathrm{H}]^{+}: 256.1507$; found 256.1505; $[\alpha]_{\mathrm{D}}{ }^{21}=-11.0\left(\mathrm{c}=0.048, \mathrm{CHCl}_{3}\right)$.

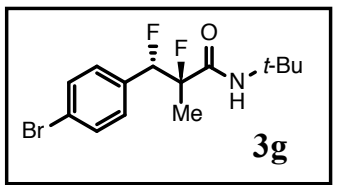

Prepared according to General Procedure B using $2 \mathrm{~g}$ (296 mg, $1.00 \mathrm{mmol})$. Analysis of the crude product mixture by ${ }^{19} \mathrm{~F}$ NMR indicated a 1,2:1,1 ratio of $>100: 1.0$. After workup, the crude residue was purified by silica gel chromatography ( 2 to $10 \%$ diethyl ether in hexanes) to give $\mathbf{3 g}(289 \mathrm{mg}, 83 \%)$ as a white solid. $\mathbf{3 g}$ was determined to be of $98 \%$ e.e by chiral HPLC analysis (AD-H, $1 \mathrm{~mL} / \mathrm{min}, 2 \%$ IPA/hexanes, $220 \mathrm{~nm}, \mathrm{t}_{\mathrm{R}}($ major $)=6.280 \mathrm{~min}, \mathrm{t}_{\mathrm{R}}($ minor $\left.)=8.468 \mathrm{~min}\right)$.

${ }^{1} \mathrm{H}$ NMR $\left(500 \mathrm{MHz}, \mathrm{CDCl}_{3}\right) \delta 7.50-7.46(\mathrm{~m}, 2 \mathrm{H}), 7.25-7.21(\mathrm{~m}, 2 \mathrm{H}), 5.81(\mathrm{br} \mathrm{s}, 1 \mathrm{H}), 5.67(\mathrm{dd}, J=44.1$, $25.5 \mathrm{~Hz}, 1 \mathrm{H}), 1.75(\mathrm{dd}, J=22.5,1.7 \mathrm{~Hz}, 3 \mathrm{H}), 1.18(\mathrm{~s}, 9 \mathrm{H}) ;{ }^{13} \mathrm{C} \mathrm{NMR}\left(125.7 \mathrm{MHz}, \mathrm{CDCl}_{3}\right) \delta 168.3(\mathrm{dd}, J=$ 17.7, $7.2 \mathrm{~Hz}), 133.3(\mathrm{~d}, J=22.1 \mathrm{~Hz}), 131.3,129.1(\mathrm{~d}, J=6.6 \mathrm{~Hz}), 123.2(\mathrm{~d}, J=1.9 \mathrm{~Hz}), 97.7(\mathrm{dd}, J=197.5$, $22.4 \mathrm{~Hz}), 93.9(\mathrm{dd}, J=184.5,19.2 \mathrm{~Hz}), 51.4,28.5,20.2(\mathrm{dd}, J=24.5,5.0 \mathrm{~Hz}) ;{ }^{19} \mathrm{~F} \mathrm{NMR}\left(470.4 \mathrm{MHz}, \mathrm{CDCl}_{3}\right)$ $\delta-169.33--169.89(\mathrm{~m}, 1 \mathrm{~F}),-197.08$ (dd, $J=44.1,10.1 \mathrm{~Hz}, 1 \mathrm{~F})$; FTIR (thin film) $v 3440,2972,1682,1528$, $1012,777 \mathrm{~cm}^{-1}$; HRMS (ESI-TOF) Calc'd for $\mathrm{C}_{14} \mathrm{H}_{19} \mathrm{BrF}_{2} \mathrm{NO}[\mathrm{M}+\mathrm{H}]^{+}: 334.0613$; found 334.0610; $[\alpha]_{\mathrm{D}}{ }^{22}=-$ $0.7\left(\mathrm{c}=0.19, \mathrm{CHCl}_{3}\right)$. 


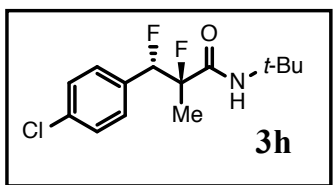

Prepared according to General Procedure B using $\mathbf{2 h}(252 \mathrm{mg}, 1.00 \mathrm{mmol})$. Analysis of the crude product mixture by ${ }^{19} \mathrm{~F}$ NMR indicated a $1,2: 1,1$ ratio of $86: 1.0$. After workup, the crude residue was purified by silica gel chromatography ( 2 to $6 \%$ diethyl ether in hexanes) to give $\mathbf{3 h}(243 \mathrm{mg}, 84 \%)$ as a white solid. $\mathbf{3 h}$ was determined to be of $97 \%$ e.e. by chiral $\mathrm{GC}$ analysis $\left(\beta\right.$-Cyclosil, $40 \rightarrow 200{ }^{\circ} \mathrm{C}, 0.2 \% \mathrm{~min}, 14 \mathrm{psi}$, $\mathrm{t}_{\mathrm{R}}($ minor $)=402.049 \mathrm{~min}, \mathrm{t}_{\mathrm{R}}($ major $\left.)=403.697 \mathrm{~min}\right)$.

${ }^{1} \mathrm{H}$ NMR (500 MHz, $\left.\mathrm{CDCl}_{3}\right) \delta 7.35-7.28(\mathrm{~m}, 4 \mathrm{H}), 5.81(\mathrm{br} \mathrm{s}, 1 \mathrm{H}), 5.69(\mathrm{dd}, J=44.1,25.5 \mathrm{~Hz}, 1 \mathrm{H}), 1.76(\mathrm{dd}$, $J=22.5,1.7 \mathrm{~Hz}, 3 \mathrm{H}), 1.17(\mathrm{~s}, 9 \mathrm{H}) ;{ }^{13} \mathrm{C} \mathrm{NMR}\left(125.7 \mathrm{MHz}, \mathrm{CDCl}_{3}\right) \delta 168.3(\mathrm{dd}, J=17.7,7.2 \mathrm{~Hz}), 135.0(\mathrm{~d}, J=$ $1.8 \mathrm{~Hz}), 132.8(\mathrm{~d}, J=22.4 \mathrm{~Hz}), 128.9(\mathrm{~d}, J=6.8 \mathrm{~Hz}), 128.3,97.8(\mathrm{dd}, J=197.5,22.6 \mathrm{~Hz}), 93.9(\mathrm{dd}, J=184.3$, $19.2 \mathrm{~Hz}), 51.4,28.4,20.2(\mathrm{dd}, J=24.5,5.1 \mathrm{~Hz}) ;{ }^{19} \mathrm{~F}$ NMR $\left(470.4 \mathrm{MHz}, \mathrm{CDCl}_{3}\right) \delta-169.44--169.74(\mathrm{~m}, 1 \mathrm{~F}),-$ 196.72 (dd, $J=44.2,9.9 \mathrm{~Hz}, 1 \mathrm{~F})$; FTIR (thin film) $v 3441,2969,1681,1530,779 \mathrm{~cm}^{-1}$; HRMS (ESI-TOF) Calc'd for $\mathrm{C}_{14} \mathrm{H}_{19} \mathrm{ClF}_{2} \mathrm{NO}[\mathrm{M}+\mathrm{H}]^{+}: 290.1118$; found $290.1115 ;[\alpha]_{\mathrm{D}}{ }^{21}=-2.2\left(\mathrm{c}=0.14, \mathrm{CHCl}_{3}\right)$.

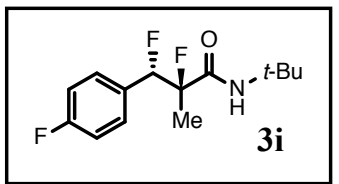

Prepared according to General Procedure B using 2i (235 mg, $1.00 \mathrm{mmol})$. Analysis of the crude product mixture by ${ }^{19} \mathrm{~F}$ NMR indicated a 1,2:1,1 ratio of 16:1.0. After workup, the crude residue was purified by silica gel chromatography ( 2 to $8 \%$ diethyl ether in hexanes) to give $\mathbf{3 i}$ (197 $\mathrm{mg}, 72 \%)$ as a white solid. $3 \mathbf{i}$ was determined to be of $98 \%$ e.e. by chiral GC analysis $\left(\beta\right.$-Cyclosil, $40 \rightarrow 200{ }^{\circ} \mathrm{C}, 1.5 \% \mathrm{~min}, 14 \mathrm{psi}$, $\mathrm{t}_{\mathrm{R}}($ minor $)=57.213 \mathrm{~min}, \mathrm{t}_{\mathrm{R}}($ major $\left.)=57.730 \mathrm{~min}\right)$.

${ }^{1} \mathrm{H}$ NMR $\left(500 \mathrm{MHz}, \mathrm{CDCl}_{3}\right) \delta 7.35(\mathrm{dd}, J=8.1,5.6 \mathrm{~Hz}, 2 \mathrm{H}), 7.08-6.99(\mathrm{~m}, 2 \mathrm{H}), 5.79(\mathrm{br} \mathrm{s}, 1 \mathrm{H}), 5.68(\mathrm{dd}, J$ $=43.9,25.6 \mathrm{~Hz}, 1 \mathrm{H}), 1.76(\mathrm{dd}, J=22.5,1.7 \mathrm{~Hz}, 3 \mathrm{H}), 1.15(\mathrm{~s}, 9 \mathrm{H}) ;{ }^{13} \mathrm{C} \mathrm{NMR}\left(125.7 \mathrm{MHz}, \mathrm{CDCl}_{3}\right) \delta 168.4(\mathrm{dd}$, $J=17.7,7.2 \mathrm{~Hz}), 163.2(\mathrm{~d}, J=247.8 \mathrm{~Hz}), 130.1(\mathrm{~d}, J=22.8 \mathrm{~Hz}), 129.8-129.3(\mathrm{~m}), 115.1(\mathrm{~d}, J=21.7 \mathrm{~Hz})$, $97.9(\mathrm{dd}, J=197.5,22.6 \mathrm{~Hz}), 94.0(\mathrm{dd}, J=184.0,18.9 \mathrm{~Hz}), 51.3,28.4,20.2(\mathrm{dd}, J=24.7,4.8 \mathrm{~Hz}) ;{ }^{19} \mathrm{~F} \mathrm{NMR}$ $\left(470.4 \mathrm{MHz}, \mathrm{CDCl}_{3}\right) \delta-112.66(\mathrm{ttd}, J=8.1,5.3,2.5 \mathrm{~Hz}, 1 \mathrm{~F}),-169.70--170.01(\mathrm{~m}, 1 \mathrm{~F}),-195.10(\mathrm{dd}, J=44.0$, $10.0 \mathrm{~Hz}, 1 \mathrm{~F}$ ); FTIR (thin film) v 3388, 2984, 1663, 1532, 1228, $788 \mathrm{~cm}^{-1}$; HRMS (ESI-TOF) Calc'd for $\mathrm{C}_{14} \mathrm{H}_{19} \mathrm{~F}_{3} \mathrm{NO}[\mathrm{M}+\mathrm{H}]^{+}: 274.1413$; found 274.1413; $[\alpha]_{\mathrm{D}}{ }^{22}=-8.1\left(\mathrm{c}=0.11, \mathrm{CHCl}_{3}\right)$.

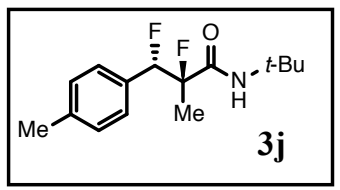

Prepared according to General Procedure B using $2 \mathbf{j}$ (231 $\mathrm{mg}, 1.00 \mathrm{mmol})$ and with the following modification: 25 equiv hydrogen fluoride were used. Analysis of the crude product mixture by ${ }^{19} \mathrm{~F}$ NMR indicated a 1,2:1,1 ratio of 2.2:1.0. After workup, the crude residue was purified by silica gel chromatography (1 to $7 \%$ diethyl ether in hexanes) to give $\mathbf{3 j}$ (107 $\mathrm{mg}, 40 \%$ ) as a white solid. $\mathbf{3 j}$ was determined to be of $98 \%$ e.e by chiral HPLC analysis (AD-H, $1 \mathrm{~mL} / \mathrm{min}, 2 \% \mathrm{IPA} /$ hexanes, $220 \mathrm{~nm}$, $\mathrm{t}_{\mathrm{R}}$ (major) $=6.029 \mathrm{~min}$, $\mathrm{t}_{\mathrm{R}}($ minor $)=$ $7.590 \mathrm{~min})$.

${ }^{1} \mathrm{H}$ NMR $\left(500 \mathrm{MHz}, \mathrm{CDCl}_{3}\right) \delta 7.24(\mathrm{~d}, J=7.9 \mathrm{~Hz}, 2 \mathrm{H}), 7.14(\mathrm{~d}, J=7.8 \mathrm{~Hz}, 2 \mathrm{H}), 5.80(\mathrm{br} \mathrm{s}, 1 \mathrm{H}), 5.65(\mathrm{dd}, J=$ 44.0, $25.8 \mathrm{~Hz}, 2 \mathrm{H}), 2.35(\mathrm{~d}, J=0.9 \mathrm{~Hz}, 3 \mathrm{H}), 1.75(\mathrm{dd}, J=22.4,1.7 \mathrm{~Hz}, 3 \mathrm{H}), 1.15(\mathrm{~s}, 9 \mathrm{H}) ;{ }^{13} \mathrm{C}$ NMR $(125.7$ $\left.\mathrm{MHz}, \mathrm{CDCl}_{3}\right) \delta 168.57(\mathrm{dd}, J=17.6,7.1 \mathrm{~Hz}), 138.83(\mathrm{~d}, J=1.7 \mathrm{~Hz}), 131.30(\mathrm{~d}, J=22.1 \mathrm{~Hz}), 128.75,127.38$ (dd, $J=7.7,1.3 \mathrm{~Hz}), 98.04$ (dd, $J=197.0,22.7 \mathrm{~Hz}), 94.57$ (dd, $J=183.4,19.2 \mathrm{~Hz}), 51.24,28.43,21.34,20.21$ $(\mathrm{dd}, J=24.5,5.1 \mathrm{~Hz}) ;{ }^{19} \mathrm{~F}$ NMR $\left(470.4 \mathrm{MHz}, \mathrm{CDCl}_{3}\right) \delta-169.08--169.31(\mathrm{~m}, 1 \mathrm{~F}),-195.50(\mathrm{dd}, J=44.2,9.7$ $\mathrm{Hz}, 1 \mathrm{~F}$ ); FTIR (thin film) v 3441, 2965, 1684, 1526, 1034, $777 \mathrm{~cm}^{-1}$; HRMS (ESI-TOF) Calc'd for $\mathrm{C}_{15} \mathrm{H}_{22} \mathrm{~F}_{2} \mathrm{NO}[\mathrm{M}+\mathrm{H}]^{+}: 270.1664$; found $270.1661 ;[\alpha]_{\mathrm{D}}^{22}=-5.8\left(\mathrm{c}=0.053, \mathrm{CHCl}_{3}\right)$.

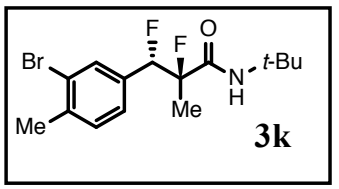

Prepared according to General Procedure B using 2k (310 mg, $1.00 \mathrm{mmol})$. Analysis of the crude product mixture by ${ }^{19} \mathrm{~F}$ NMR indicated a 1,2:1,1 ratio of 79:1.0. After workup, the crude residue was purified by silica gel chromatography (2 to $10 \%$ diethyl ether in hexanes) to give $\mathbf{3 k}(289 \mathrm{mg}, 83 \%)$ as a white solid. $\mathbf{3 k}$ was determined to be of $98 \%$ e.e. by chiral GC analysis ( $\beta$-Cyclosil, $40 \rightarrow 200{ }^{\circ} \mathrm{C}, 1.5$ $\%$ min, 14 psi, $\mathrm{t}_{\mathrm{R}}($ minor $)=82.246 \mathrm{~min}, \mathrm{t}_{\mathrm{R}}($ major $\left.)=82.683 \mathrm{~min}\right)$.

${ }^{1} \mathrm{H}$ NMR $\left(500 \mathrm{MHz}, \mathrm{CDCl}_{3}\right) \delta 7.51(\mathrm{~s}, 1 \mathrm{H}), 7.20(\mathrm{dd}, J=1.3,0.7 \mathrm{~Hz}, 2 \mathrm{H}), 5.83(\mathrm{~s}, 1 \mathrm{H}), 5.66(\mathrm{dd}, J=44.1$, $25.6 \mathrm{~Hz}, 1 \mathrm{H}), 2.39(\mathrm{~d}, J=1.0 \mathrm{~Hz}, 3 \mathrm{H}), 1.75(\mathrm{dd}, J=22.5,1.8 \mathrm{~Hz}, 3 \mathrm{H}), 1.19$ (s, 9H); ${ }^{13} \mathrm{C}$ NMR $(125.7 \mathrm{MHz}$, 
$\left.\mathrm{CDCl}_{3}\right) \delta 168.4(\mathrm{dd}, J=17.7,7.3 \mathrm{~Hz}), 138.7,133.7(\mathrm{~d}, J=22.3 \mathrm{~Hz}), 131.0(\mathrm{~d}, J=7.2 \mathrm{~Hz}), 130.4,126.2(\mathrm{~d}, J=$ 7.0 Hz), 124.6, 97.9 (dd, $J=197.5,22.5 \mathrm{~Hz}), 93.6$ (dd, $J=184.7,19.3 \mathrm{~Hz}), 51.5,28.5,22.9,20.2$ (dd, $J=24.6$, $5.3 \mathrm{~Hz}) ;{ }^{19} \mathrm{~F}$ NMR $\left(470.4 \mathrm{MHz}, \mathrm{CDCl}_{3}\right) \delta-169.23--169.52(\mathrm{~m}, 1 \mathrm{~F}),-197.31$ (dd, $\left.J=43.8,9.9 \mathrm{~Hz}, 1 \mathrm{~F}\right)$; FTIR (thin film) $v 3401,2976,1665,1531,1048,781 \mathrm{~cm}^{-1}$; HRMS (ESI-TOF) Calc'd for $\mathrm{C}_{15} \mathrm{H}_{21} \mathrm{BrF}_{2} \mathrm{NO}$ [M+H] $]^{+}$: 348.0769 ; found $348.0767 ;[\alpha]_{\mathrm{D}}^{22}=+5.0\left(\mathrm{c}=0.23, \mathrm{CHCl}_{3}\right)$.

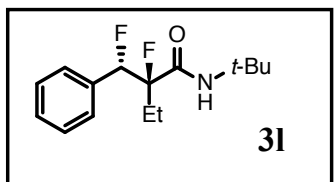

Prepared according to General Procedure B using 21 (231 mg, $1.00 \mathrm{mmol})$. Analysis of the crude product mixture by ${ }^{19} \mathrm{~F}$ NMR indicated a 1,2:1,1 ratio of $>100: 1.0$. After workup, the crude residue was purified by silica gel chromatography ( 2 to $6 \%$ diethyl ether in hexanes) to give $3 \mathrm{I}(156 \mathrm{mg}, 58 \%)$ as a colorless oil. $\mathbf{3 l}$ was determined to be of $95 \%$ e.e. by chiral $\mathrm{GC}$ analysis $\left(\beta\right.$-Cyclosil, $40 \rightarrow 200{ }^{\circ} \mathrm{C}, 1.5 \% \mathrm{~min}, 14 \mathrm{psi}$, $\mathrm{t}_{\mathrm{R}}($ minor $)=64.469$ min, $\mathrm{t}_{\mathrm{R}}($ major $\left.)=65.011 \mathrm{~min}\right)$.

${ }^{1} \mathrm{H}$ NMR $\left(500 \mathrm{MHz}, \mathrm{CDCl}_{3}\right) \delta 7.38-7.31(\mathrm{~m}, 5 \mathrm{H}), 5.78($ br s, $1 \mathrm{H}), 5.69(\mathrm{dd}, J=44.3,25.8 \mathrm{~Hz}, 1 \mathrm{H}), 2.30-$ $2.18(\mathrm{~m}, 1 \mathrm{H}), 2.18-2.09(\mathrm{~m}, 1 \mathrm{H}), 1.14(\mathrm{~s}, 9 \mathrm{H}), 0.98(\mathrm{t}, J=7.4 \mathrm{~Hz}, 3 \mathrm{H}) ;{ }^{13} \mathrm{C}$ NMR $\left(125.7 \mathrm{MHz}, \mathrm{CDCl}_{3}\right) \delta$ $167.4(\mathrm{dd}, J=17.8,7.1 \mathrm{~Hz}), 134.4(\mathrm{~d}, J=22.0 \mathrm{~Hz}), 129.0(\mathrm{~d}, J=1.8 \mathrm{~Hz}), 128.0,127.5(\mathrm{~d}, J=6.4 \mathrm{~Hz}), 100.8$ (dd, $J=200.2,21.8 \mathrm{~Hz}), 94.6(\mathrm{dd}, J=183.8,18.9 \mathrm{~Hz}), 51.4,28.4,26.4(\mathrm{dd}, J=23.2,4.4 \mathrm{~Hz}), 7.4(\mathrm{~d}, J=3.8$ $\mathrm{Hz}) ;{ }^{19} \mathrm{~F}$ NMR $\left(470.4 \mathrm{MHz}, \mathrm{CDCl}_{3}\right) \delta-181.01--181.20(\mathrm{~m}, 1 \mathrm{~F}),-196.00$ (dd, $\left.J=44.3,10.0 \mathrm{~Hz}, 1 \mathrm{~F}\right)$; FTIR (thin film) $v 3444,2973,1681,1526,1457,717 \mathrm{~cm}^{-1}$; HRMS (ESI-TOF) Calc'd for $\mathrm{C}_{15} \mathrm{H}_{22} \mathrm{~F}_{2} \mathrm{NO}[\mathrm{M}+\mathrm{H}]^{+}$: 270.1664; found 270.1663; $[\alpha]_{\mathrm{D}}^{22}=-2.5\left(\mathrm{c}=0.076, \mathrm{CHCl}_{3}\right)$.

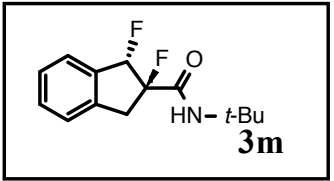

Prepared according to General Procedure B using 2m (215 mg, $1.00 \mathrm{mmol})$. Analysis of the crude product mixture by ${ }^{19} \mathrm{~F}$ NMR indicated a 1,2:1,1 ratio of $>100: 1.0$. After workup, the crude residue was purified by silica gel chromatography (5-20\% diethyl ether in hexanes) to give $\mathbf{3 m}(108 \mathrm{mg}, 43 \%)$ as a pale yellow solid. $\mathbf{3 m}$ was determined to be of $77 \%$ e.e by chiral GC analysis ( $\beta$-Cyclosil, $40 \rightarrow 200{ }^{\circ} \mathrm{C}, 1.5$ $\% / \mathrm{min}, 14 \mathrm{psi}, \mathrm{t}_{\mathrm{R}}($ minor $)=76.993 \mathrm{~min}, \mathrm{t}_{\mathrm{R}}($ major $\left.)=77.287 \mathrm{~min}\right)$.

${ }^{1} \mathrm{H}$ NMR $\left(500 \mathrm{MHz}, \mathrm{CDCl}_{3}\right) \delta$ 7.46-7.42 (m, 1H), 7.40-7.35 (m, 1H), 7.33-7.27 (m, 1H), $6.26(\mathrm{~s}, 1 \mathrm{H}), 5.99$ $(\mathrm{dd}, J=54.9,19.2 \mathrm{~Hz}, 1 \mathrm{H}), 3.93(\mathrm{dd}, J=18.2,17.1 \mathrm{~Hz}, 1 \mathrm{H}), 3.20(\mathrm{ddd}, J=23.5,17.1,1.9 \mathrm{~Hz}, 1 \mathrm{H}), 1.42(\mathrm{~s}$, $9 \mathrm{H}) ;{ }^{13} \mathrm{C}$ NMR $\left(125.7 \mathrm{MHz}, \mathrm{CDCl}_{3}\right) \delta 166.1(\mathrm{dd}, J=20.6,3.0 \mathrm{~Hz}), 141.0(\mathrm{dd}, J=5.1,2.1 \mathrm{~Hz}), 136.2(\mathrm{dd}, J=$ 19.5, $4.4 \mathrm{~Hz}), 130.7$ (d, $J=3.6 \mathrm{~Hz}), 127.8$ (d, $J=2.9 \mathrm{~Hz}), 125.6,124.9$ (d, $J=1.8 \mathrm{~Hz}), 103.8$ (dd, $J=196.5$, $22.3 \mathrm{~Hz}), 99.9(\mathrm{dd}, J=190.1,35.7 \mathrm{~Hz}), 51.9,40.1(\mathrm{~d}, J=24.5 \mathrm{~Hz}), 28.8 ;{ }^{19} \mathrm{~F}$ NMR $\left(470.4 \mathrm{MHz}, \mathrm{CDCl}_{3}\right) \delta-$ $156.56--156.72(\mathrm{~m}, 1 \mathrm{~F}),-179.96(\mathrm{dd}, J=54.5,4.9 \mathrm{~Hz}, 1 \mathrm{~F})$; FTIR (thin film) v 3439, 3351, 2969, 2932, 1686, 1526, 1227, 1018, $753 \mathrm{~cm}^{-1}$; HRMS (ESI-TOF) Calc'd for $\mathrm{C}_{14} \mathrm{H}_{18} \mathrm{~F}_{2} \mathrm{NO}[\mathrm{M}+\mathrm{H}]^{+}:$: 254.1351; found 254.1349; ; $[\alpha]_{\mathrm{D}}^{22}=51.9\left(\mathrm{c}=0.079, \mathrm{CHCl}_{3}\right)$.

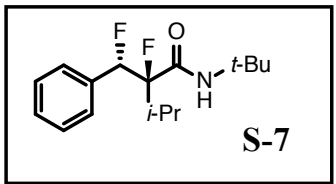

Prepared according to General Procedure B using S-6 (49.1 mg, $0.20 \mathrm{mmol})$. Analysis of the crude product mixture by ${ }^{19} \mathrm{~F}$ NMR indicated a 1,2:1,1 ratio of $>100: 1.0$. After workup, the crude residue was purified by silica gel chromatography $(10 \%$ diethyl ether in hexanes) to give S-7 $(24.7 \mathrm{mg}, 44 \%)$ as a colorless oil. S-7 was determined to be of $97 \%$ e.e by chiral $\mathrm{GC}$ analysis (CP-Chirasil-Dex CB, $40 \rightarrow 160{ }^{\circ} \mathrm{C}, 1.5 \% \mathrm{~min}$, 14 psi, $\mathrm{t}_{\mathrm{R}}($ minor $)=69.959 \mathrm{~min}, \mathrm{t}_{\mathrm{R}}($ major $\left.)=70.983 \mathrm{~min}\right)$.

${ }^{1} \mathrm{H}$ NMR $\left(500 \mathrm{MHz}, \mathrm{CDCl}_{3}\right) \delta 7.39-7.30(\mathrm{~m}, 5 \mathrm{H}), 5.95(\mathrm{dd}, J=44.1,26.9 \mathrm{~Hz}, 1 \mathrm{H}), 5.75(\mathrm{~s}, 1 \mathrm{H}), 2.51(\mathrm{dpd}, J$ $=17.5,6.9,1.4 \mathrm{~Hz}, 1 \mathrm{H}), 1.27(\mathrm{~d}, J=7.0 \mathrm{~Hz}, 3 \mathrm{H}), 1.13(\mathrm{~s}, 9 \mathrm{H}), 1.05(\mathrm{dd}, J=6.9,1.1 \mathrm{~Hz}, 3 \mathrm{H}) ;{ }^{13} \mathrm{C} \mathrm{NMR}(125.7$ $\left.\mathrm{MHz}, \mathrm{CDCl}_{3}\right) \delta 167.0(\mathrm{dd}, J=18.0,7.9 \mathrm{~Hz}), 134.6(\mathrm{~d}, J=21.8 \mathrm{~Hz}), 128.9(\mathrm{~d}, J=1.6 \mathrm{~Hz}), 128.0,127.7(\mathrm{~d}, J=$ 7.6 Hz), 101.7 (dd, $J=199.6,21.9 \mathrm{~Hz}), 93.2$ (dd, $J=183.6,18.8 \mathrm{~Hz}), 51.4,32.7$ (dd, $J=23.4,3.3 \mathrm{~Hz}$ ), 28.5, $17.9(\mathrm{~d}, J=8.6 \mathrm{~Hz}), 17.0 ;{ }^{19} \mathrm{~F}$ NMR $\left(470.4 \mathrm{MHz}, \mathrm{CDCl}_{3}\right) \delta-174.72--174.94(\mathrm{~m}),-197.81(\mathrm{dd}, J=44.1,10.0$ $\mathrm{Hz}$ ); FTIR (thin film) v 3445, 2971, 1681, 1524, 1456, 1021, $716 \mathrm{~cm}^{-1}$; HRMS (ESI-TOF) Calc'd for $\mathrm{C}_{16} \mathrm{H}_{24} \mathrm{~F}_{2} \mathrm{NO}[\mathrm{M}+\mathrm{H}]^{+}:$284.1820; found 284.1819. 


\section{Derivatization of 1,2-Difluoride Products}

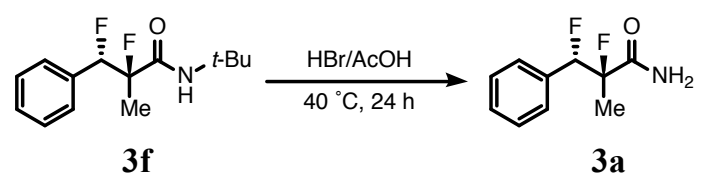

3a: To a screw-cap vial charged with $\mathbf{3 f}(51.1 \mathrm{mg}, 0.20 \mathrm{mmol})$ and a stir bar, hydrogen bromide solution (1.00 $\mathrm{mL}, 33 \mathrm{wt} . \%$ in acetic acid) was added. The vial was capped tightly with a Teflon-lined cap and warmed to 40 ${ }^{\circ} \mathrm{C}$ for $24 \mathrm{~h}$. The reaction mixture was then cooled to room temperature and diluted with ethyl acetate $(10 \mathrm{~mL})$. Saturated aqueous $\mathrm{NaHCO}_{3}$ was added slowly until the aqueous layer reached $\mathrm{pH}=8$. The layers were separated, and the organic layer was washed with water $(3 \times 5 \mathrm{~mL})$, washed with brine $(5 \mathrm{~mL})$, dried over anhydrous $\mathrm{MgSO}_{4}$, and concentrated. The resulting residue was purified by flash column chromatography on silica gel (50\% ethyl acetate in hexanes) to give $\mathbf{3 a}$ as a white solid $(35.4 \mathrm{mg}, 89 \%)$. 3a was determined to be of $96 \%$ e.e by chiral GC analysis ( $\beta$-Cyclosil, $40 \rightarrow 200{ }^{\circ} \mathrm{C}, 1.5 \% \mathrm{~min}, 14 \mathrm{psi}, \mathrm{t}_{\mathrm{R}}$ (major) $=67.968 \mathrm{~min}$, $\mathrm{t}_{\mathrm{R}}($ minor $\left.)=68.946 \mathrm{~min}\right)$.

${ }^{1} \mathrm{H}$ NMR $\left(500 \mathrm{MHz}, \mathrm{CDCl}_{3}\right) \delta 7.40-7.33(\mathrm{~m}, 5 \mathrm{H}), 5.96(\mathrm{br} \mathrm{s}, 1 \mathrm{H}), 5.71(\mathrm{dd}, J=44.1,25.2 \mathrm{~Hz}, 1 \mathrm{H}), 5.19(\mathrm{br}$ s, $1 \mathrm{H}), 1.81(\mathrm{dd}, J=22.3,1.7 \mathrm{~Hz}, 3 \mathrm{H}) ;{ }^{13} \mathrm{C}$ NMR $\left(125.7 \mathrm{MHz}, \mathrm{DMSO}-d_{6}\right) \delta 171.0(\mathrm{dd}, J=21.2,6.8 \mathrm{~Hz}), 134.4$ $(\mathrm{d}, J=21.5 \mathrm{~Hz}), 128.9,128.0,127.3(\mathrm{~d}, J=7.2 \mathrm{~Hz}), 96.9(\mathrm{dd}, J=196.1,22.6 \mathrm{~Hz}), 94.2(\mathrm{dd}, J=179.5,19.6$ $\mathrm{Hz}), 20.4(\mathrm{dd}, J=25.2,3.9 \mathrm{~Hz}) ;{ }^{19} \mathrm{~F}$ NMR $\left(470.4 \mathrm{MHz}, \mathrm{CDCl}_{3}\right) \delta-168.84--169.24(\mathrm{~m}, 1 \mathrm{~F}),-195.80(\mathrm{dd}, J=$ 44.1, 9.7 Hz, 1F); FTIR (thin film) v 3394, 3189, 1661, 1639, 1112, 1038, $674 \mathrm{~cm}^{-1}$; HRMS (ESI-TOF) Calc'd for $\mathrm{C}_{10} \mathrm{H}_{12} \mathrm{~F}_{2} \mathrm{NO}[\mathrm{M}+\mathrm{H}]^{+}: 200.0881$; found 200.0880; $[\alpha]_{\mathrm{D}}^{22}=-103\left(\mathrm{c}=0.0062, \mathrm{CHCl}_{3}\right)$.

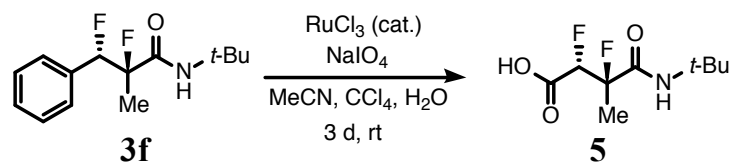

5: A catalytic amount of ruthenium trichloride hydrate and sodium periodate ( $770 \mathrm{mg}, 3.6 \mathrm{mmol}, 18$ equiv) were added to a vigorously stirred solution of $\mathbf{3 f}(51.1 \mathrm{mg}, 0.20 \mathrm{mmol}, 1.0$ equiv) in carbon tetrachloride $(0.75$ $\mathrm{mL})$, acetonitrile $(0.75 \mathrm{~mL})$, and water $(2.0 \mathrm{~mL})$. The reaction vessel was equipped with a vent needle, and the biphasic mixture was stirred for $72 \mathrm{~h}$ at room temperature. After $72 \mathrm{~h}$, the reaction mixture was cooled to $0{ }^{\circ} \mathrm{C}$ and diethyl ether $(10 \mathrm{~mL})$ was added. The organic layer was separated, and the aqueous layer was extracted with diethyl ether $(3 \times 5 \mathrm{~mL})$. The combined organic layers were dried over anhydrous $\mathrm{MgSO}_{4}$ and concentrated under reduced pressure to afford $5(33.1 \mathrm{mg}, 74 \%)$ as a tan solid. 5 was determined to be of $96 \%$ e.e. by chiral GC analysis of its corresponding methyl ester S-8. To prepare methyl ester S-8, a solution of (diazomethyl)trimethylsilane (2.0 M in diethyl ether, $16.0 \mu \mathrm{L}, 0.032 \mathrm{mmol}, 1.5$ equiv) was added dropwise via syringe to a stirred solution of $5(5.0 \mathrm{mg}, 0.022 \mathrm{mmol}, 1$ equiv) in benzene $(150 \mu \mathrm{L})$ and methanol $(43 \mu \mathrm{L})$ at room temperature. After 30 minutes, acetic acid $(5 \mu \mathrm{L})$ was added dropwise via syringe to quench the unreacted (diazomethyl)trimethylsilane, and an aliquot of the reaction mixture was subjected to chiral GC analysis $\left(\beta\right.$-Cyclosil, $40 \rightarrow 200{ }^{\circ} \mathrm{C}, 1 \% \mathrm{~min}, 20 \mathrm{psi}, \mathrm{t}_{\mathrm{R}}$ (major) $=53.977 \mathrm{~min}, \mathrm{t}_{\mathrm{R}}($ minor $\left.)=54.311 \mathrm{~min}\right)$.

${ }^{1} \mathrm{H}$ NMR $\left(500 \mathrm{MHz}, \mathrm{CDCl}_{3}\right) \delta 6.29(\mathrm{br} \mathrm{s}, 1 \mathrm{H}), 5.30(\mathrm{dd}, J=47.7,24.5 \mathrm{~Hz}, 1 \mathrm{H}), 1.72(\mathrm{dd}, J=23.0,1.9 \mathrm{~Hz}$, $3 \mathrm{H}), 1.40(\mathrm{~s}, 9 \mathrm{H}) ;{ }^{13} \mathrm{C}$ NMR $\left(125.7 \mathrm{MHz}\right.$, acetone- $\left.d_{6}\right) \delta 168.5(\mathrm{dd}, J=18.7,6.4 \mathrm{~Hz}), 166.8(\mathrm{dd}, J=25.7,5.7$ $\mathrm{Hz}), 97.8(\mathrm{dd}, J=195.4,20.7 \mathrm{~Hz}), 91.0(\mathrm{dd}, J=192.2,22.4 \mathrm{~Hz}), 51.9,28.7,20.8(\mathrm{dd}, J=23.9,7.6 \mathrm{~Hz}) ;{ }^{19} \mathrm{~F}$ NMR (470.4 MHz, DMSO- $\left.d_{6}\right) \delta-161.51(\mathrm{pd}, J=23.5,11.0 \mathrm{~Hz}, 1 \mathrm{~F}),-202.93$ (dd, $\left.J=46.4,11.6 \mathrm{~Hz}, 1 \mathrm{~F}\right)$; FTIR (thin film) $\vee$ 3434, 2972, 1758, 1654, 1540, 1230, 1221, 1191, $1083 \mathrm{~cm}^{-1}$; HRMS (ESI-TOF) Calc'd for $\mathrm{C}_{9} \mathrm{H}_{16} \mathrm{~F}_{2} \mathrm{NO}_{3}[\mathrm{M}+\mathrm{H}]^{+}: 224.1093$; found 224.1092; $[\alpha]_{\mathrm{D}}^{22}=3.7\left(\mathrm{c}=0.029, \mathrm{CHCl}_{3}\right)$. 


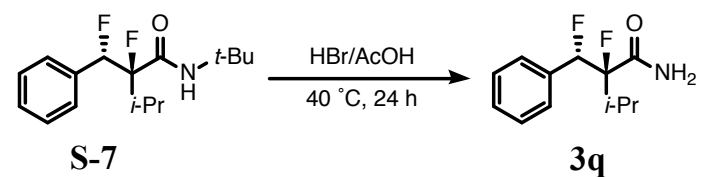

3q: To a screw-cap vial charged with S-7 $(10.0 \mathrm{mg}, 0.035 \mathrm{mmol})$ and a stir bar, hydrogen bromide solution $\left(0.50 \mathrm{~mL}, 33 \mathrm{wt} . \%\right.$ in acetic acid) was added. The vial was capped tightly and warmed to $40{ }^{\circ} \mathrm{C}$ for $24 \mathrm{~h}$. The reaction mixture was then cooled to room temperature and diluted with ethyl acetate. Saturated aqueous $\mathrm{NaHCO}_{3}$ was added slowly until the aqueous layer reached $\mathrm{pH}=8$. The layers were separated, and the organic layer was washed with water $(3 \times 5 \mathrm{~mL})$, washed with brine $(5 \mathrm{~mL})$, dried over anhydrous $\mathrm{MgSO}_{4}$, and concentrated. The resulting residue was purified by flash column chromatography on silica gel (0 to $10 \%$ diethyl ether in dichloromethane) to give $\mathbf{3 q}$ as a white solid. The spectral data for $\mathbf{3 q}$ were in accordance with the literature. ${ }^{6}[\alpha]_{\mathrm{D}}{ }^{21}=+14.3\left(\mathrm{c}=0.00275, \mathrm{CHCl}_{3}\right)$. The absolute stereochemistry of $\mathbf{3 q}$ was assigned by comparison of the sign of the optical rotation with the literature value. The assignment of absolute stereochemistry was confirmed by verifying that the major and minor enantiomer of product eluted in the same order as previously reported $\left(\beta\right.$-Cyclosil, $120 \rightarrow 200{ }^{\circ} \mathrm{C}, 1 \% \mathrm{~min}, 7 \mathrm{psi}, \mathrm{t}_{\mathrm{R}}($ major $)=46.538 \mathrm{~min}, \mathrm{t}_{\mathrm{R}}($ minor $)=$ $47.017 \mathrm{~min}){ }^{6}$ 


\section{Measurement of Product Ratios for Selected Cinnamamides}

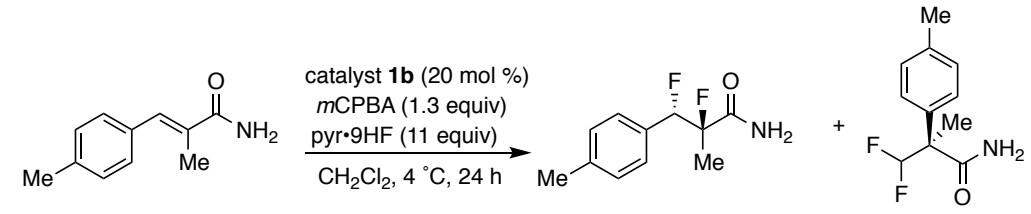

The reaction was conducted according to General Procedure B using $(E)$-2-methyl-3-( $p$-tolyl)acrylamide ${ }^{7}$ $(175.2,1.00 \mathrm{mmol})$ and with the following modifications: 100 equiv of hydrogen fluoride were used, and the reaction was quenched after $72 \mathrm{~h}$. Analysis of the crude product mixture by ${ }^{19} \mathrm{~F}$ NMR indicated a 1,2:1,1 ratio of $1.0: 36$.

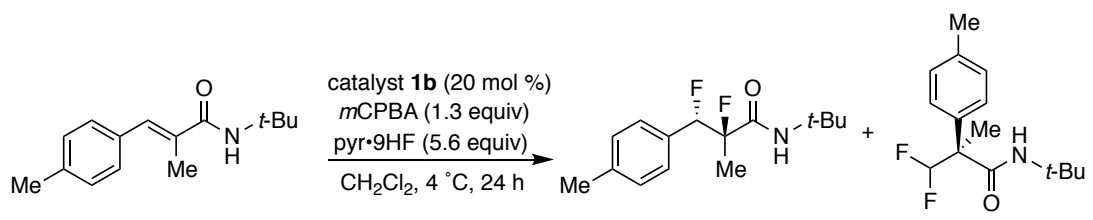

The reaction was conducted according to General Procedure B using $\mathbf{2 j}$ ( $12.9 \mathrm{mg}, 0.056 \mathrm{mmol})$. Analysis of the crude product mixture by ${ }^{19} \mathrm{~F}$ NMR indicated a 1,2:1,1 ratio of 1.4:1.0.

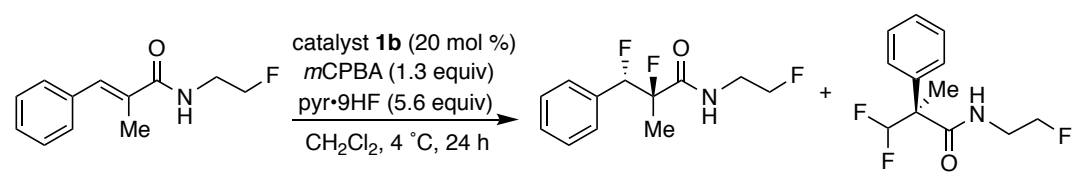

The reaction was conducted according to General Procedure B using $2 \mathbf{n}(207.3 \mathrm{mg}, 1.00 \mathrm{mmol})$. Analysis of the crude product mixture by ${ }^{19} \mathrm{~F}$ NMR indicated a 1,2:1,1 ratio of 3.4:1.0.

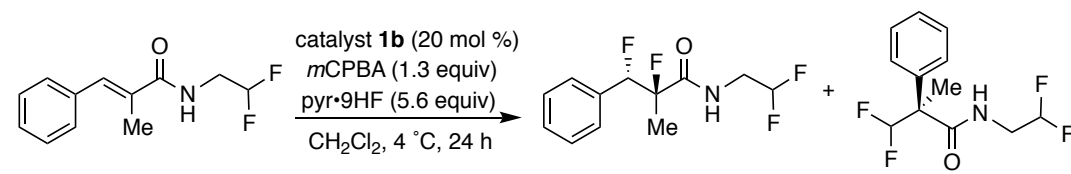

The reaction was conducted according to General Procedure B using $20(225.2 \mathrm{mg}, 1.00 \mathrm{mmol})$. Analysis of the crude product mixture by ${ }^{19} \mathrm{~F}$ NMR indicated a 1,2:1,1 ratio of 2.1:1.0.

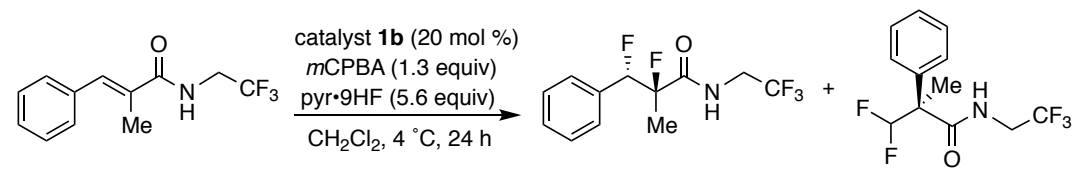

The reaction was conducted according to General Procedure B using 2p (243.2 mg, $1.00 \mathrm{mmol})$. Analysis of the crude product mixture by ${ }^{19} \mathrm{~F}$ NMR indicated a 1,2:1,1 ratio of 1.1:1.0. 


\section{Correlations Between Product Ratios and Steric and Electronic Parameters for Amide $\mathrm{N}$-Substituents}

Steric and electronic parameters for the amide $N$-substituents of $\mathbf{2 a}-\mathbf{f}$ were plotted versus the product ratio $(\ln (1,2: 1,1))$ obtained for each substrate.

Chart 1. Correlation with Taft Steric Parameter ${ }^{8,9}$

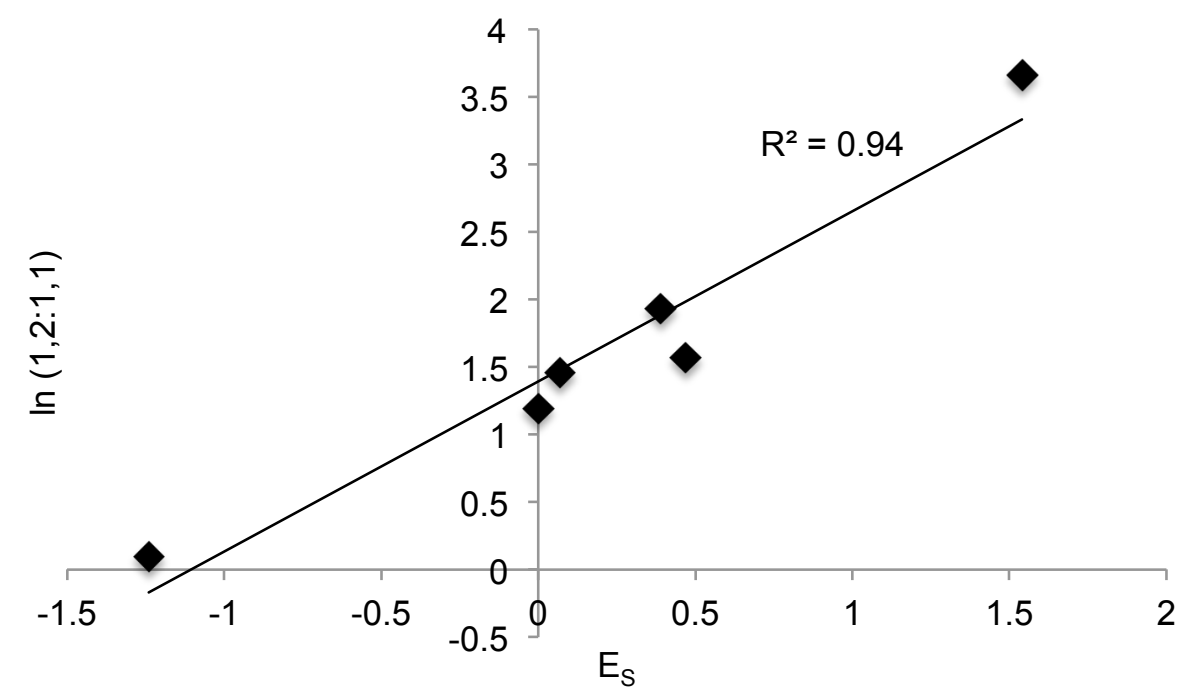

Chart 2. Correlation with A-Value ${ }^{10}$

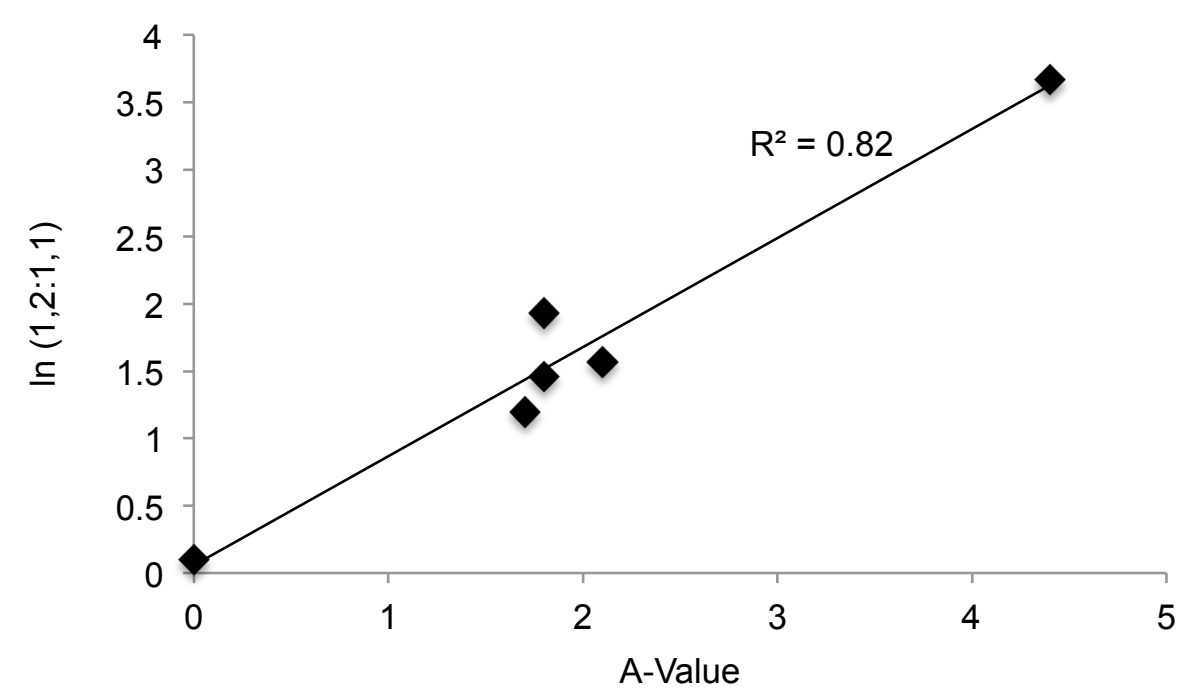


Chart 3. Correlation with Sterimol L ${ }^{11}$

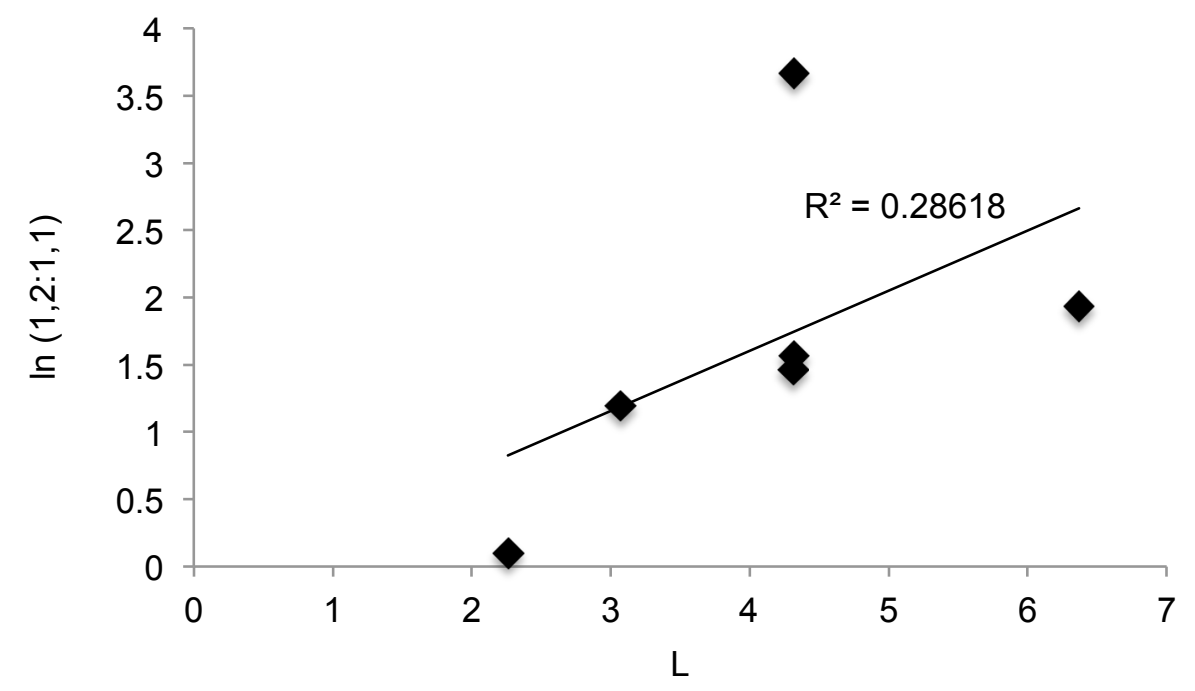

Chart 4. Correlation with Sterimol B ${ }_{1}^{11}$

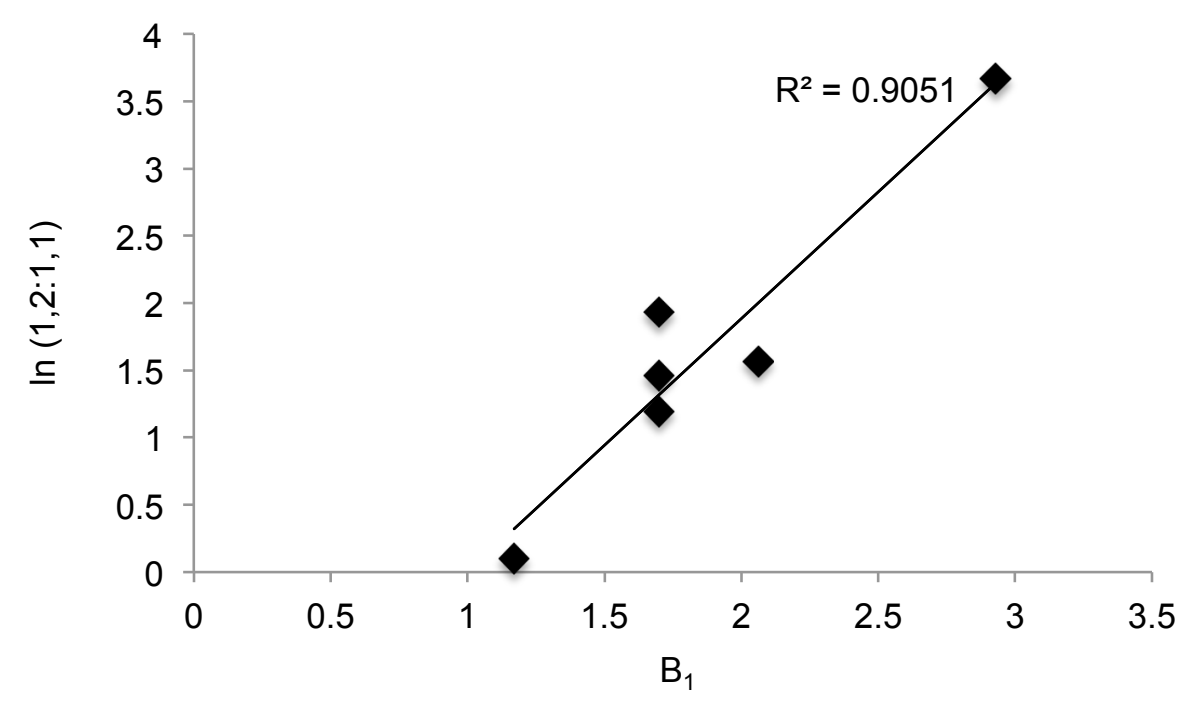


Chart 5. Correlation with Sterimol $\mathrm{B}_{5}{ }^{11}$

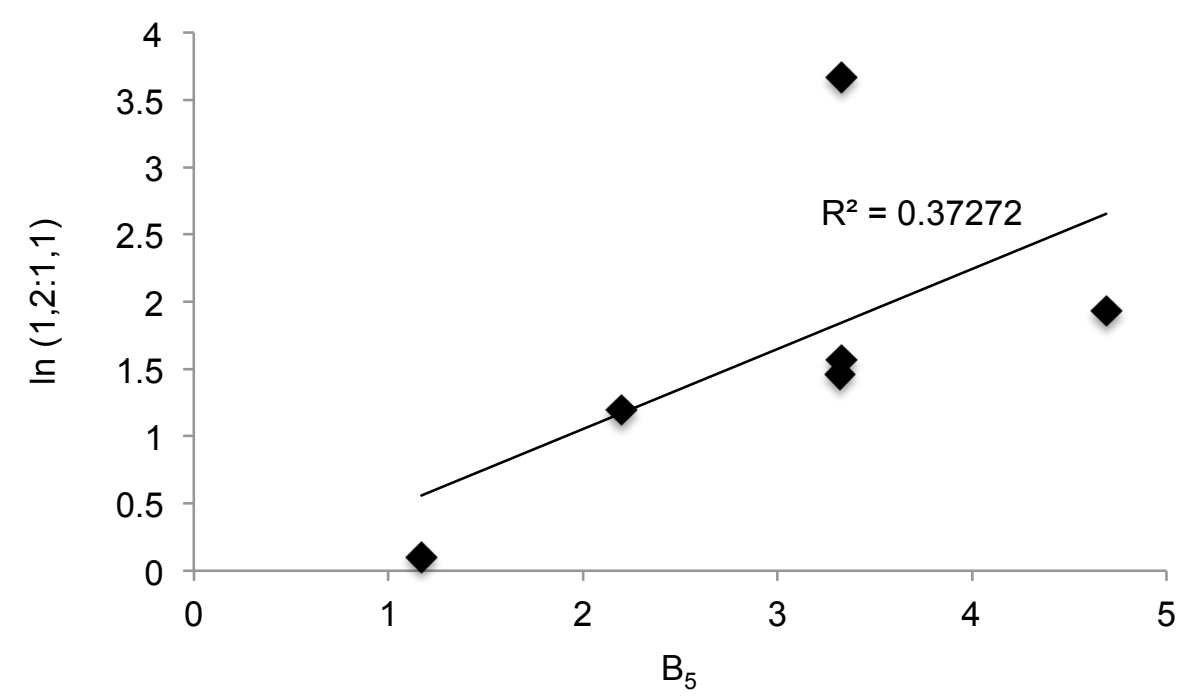

Chart 6. Correlation with Amide Carbonyl Stretching Absorption ${ }^{12}$

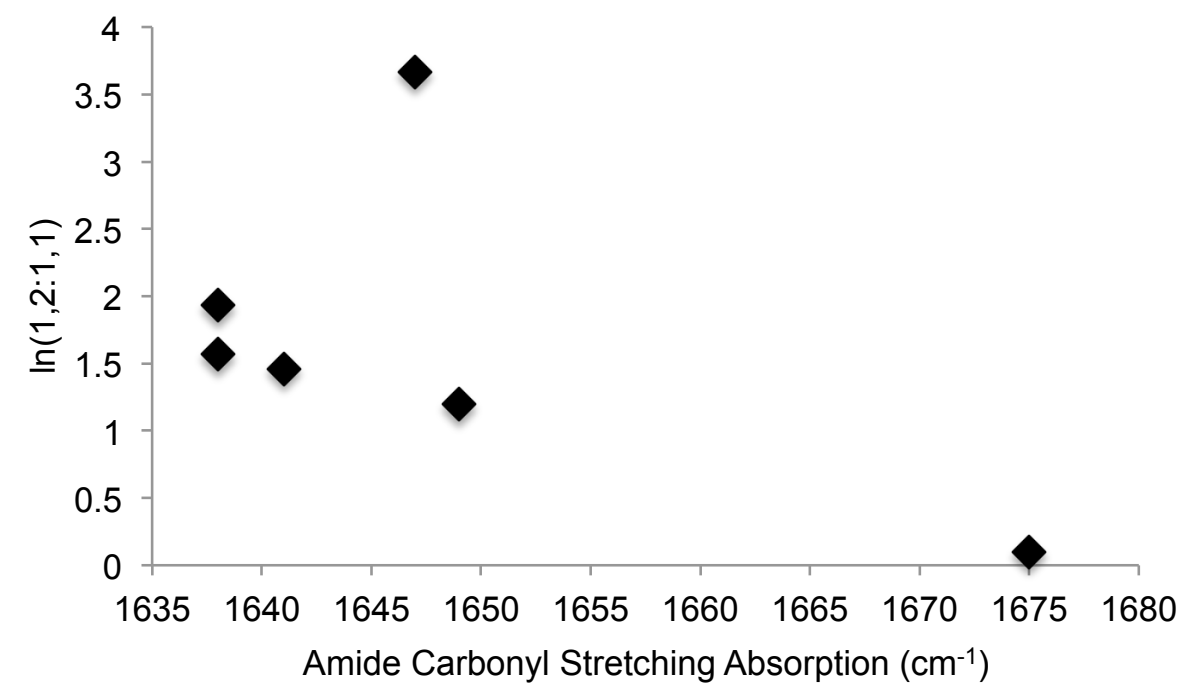




\section{Chiral GC and HPLC Traces of 1,2-Difluoride Products and Derivatives}

$\beta$-Cyclosil, $40 \rightarrow 200{ }^{\circ} \mathrm{C}, 1 \% \mathrm{~min}, 7 \mathrm{psi}$
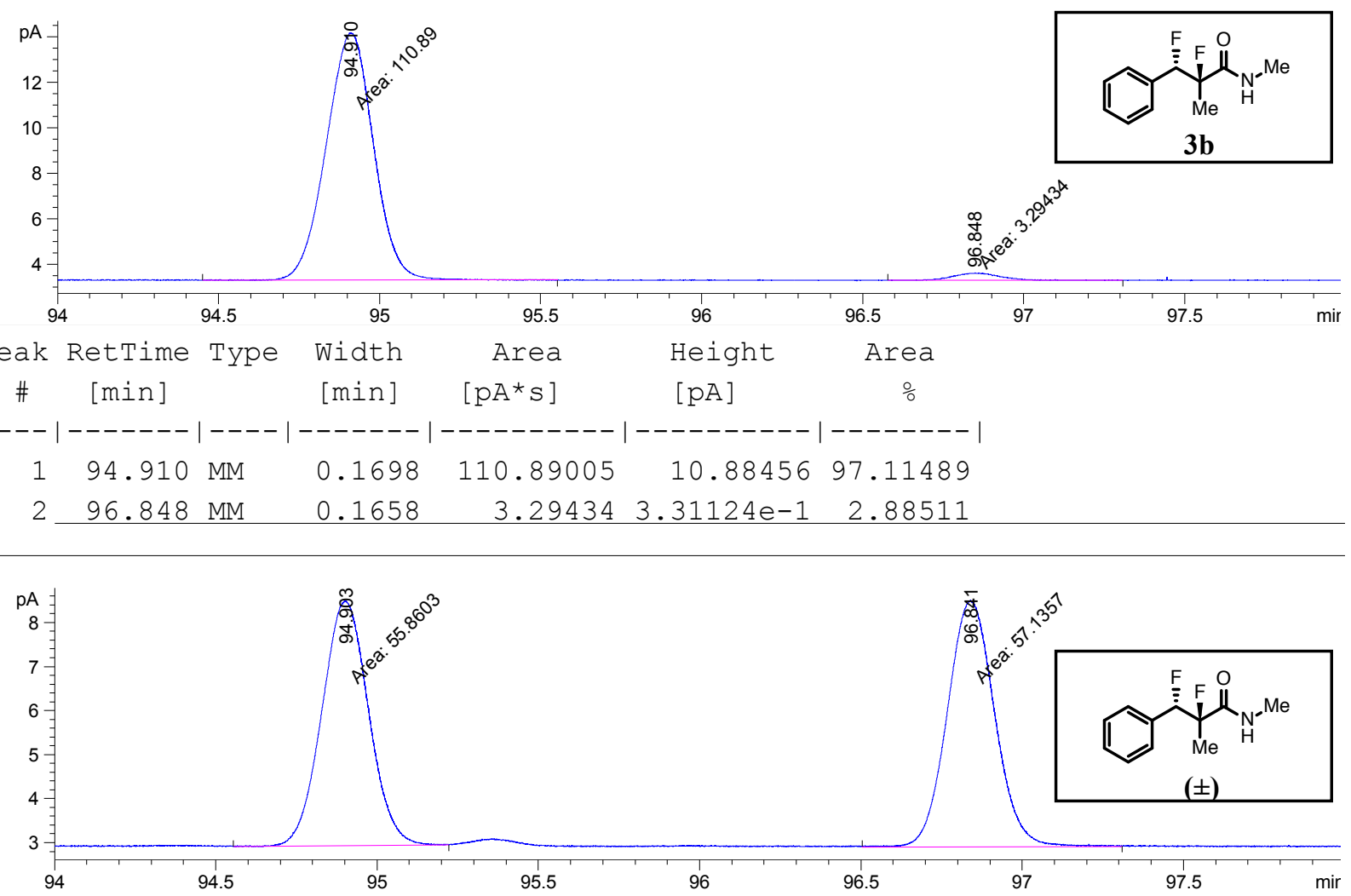

\begin{tabular}{|c|c|c|c|c|c|c|}
\hline $\begin{array}{c}\text { Peak } \\
\#\end{array}$ & $\begin{array}{c}\text { RetTime } \\
\text { [min] }\end{array}$ & Type & $\begin{array}{l}\text { Width } \\
\text { [min] }\end{array}$ & $\begin{array}{r}\text { Area } \\
{\left[\mathrm{pA}^{*} \mathrm{~s}\right]}\end{array}$ & $\begin{array}{l}\text { Height } \\
{[\mathrm{pA}]}\end{array}$ & $\begin{array}{c}\text { Area } \\
\frac{\circ}{0}\end{array}$ \\
\hline & & & & $-------\mid$ & & \\
\hline 1 & 94. & MM & 0.1675 & 55.86035 & 5.55951 & 3568 \\
\hline 2 & 96.841 & MM & 0.1704 & 57.13567 & 5.58984 & 50.56432 \\
\hline
\end{tabular}


$\beta$-Cyclosil, $40 \rightarrow 200{ }^{\circ} \mathrm{C}, 1.5^{\circ} / \mathrm{min}, 14 \mathrm{psi}$

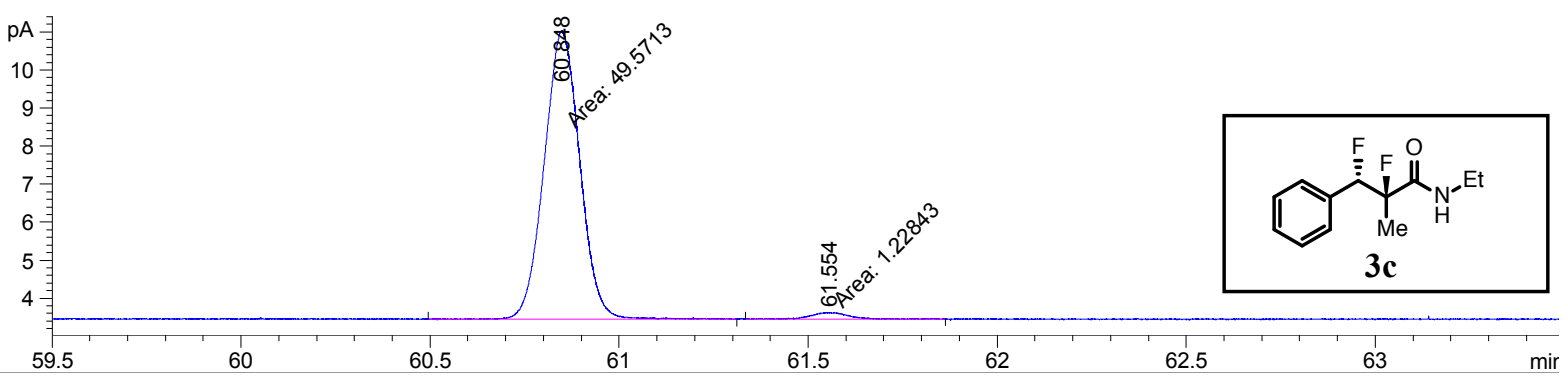

\begin{tabular}{|c|c|c|c|c|c|c|}
\hline $\begin{array}{c}\text { Peak } \\
\#\end{array}$ & $\begin{array}{c}\text { RetTime } \\
\text { [min] }\end{array}$ & Type & $\begin{array}{l}\text { Width } \\
\text { [min] }\end{array}$ & $\begin{array}{r}\text { Area } \\
{\left[\mathrm{pA}^{\star} \mathrm{s}\right]}\end{array}$ & $\begin{array}{l}\text { Height } \\
{[\mathrm{pA}]}\end{array}$ & $\begin{array}{c}\text { Area } \\
\frac{\circ}{0}\end{array}$ \\
\hline-- & ----- & & & --------- & 1 & \\
\hline 1 & 60.848 & $\mathrm{MF}$ & 0.1089 & 49.57135 & 58576 & 97.58182 \\
\hline 2 & 61.554 & FM & 0.1179 & 1.22843 & $.73609 e-1$ & 2.41818 \\
\hline
\end{tabular}

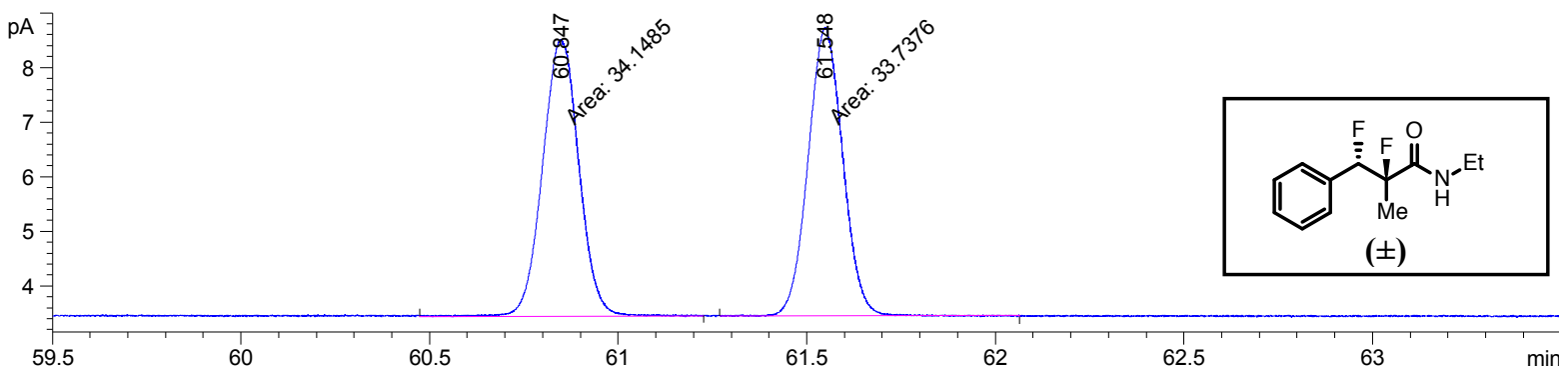

\begin{tabular}{|c|c|c|c|c|c|c|}
\hline $\begin{array}{c}\text { Peak } \\
\quad \#\end{array}$ & $\begin{array}{c}\text { RetTime } \\
\text { [min] }\end{array}$ & Type & $\begin{array}{l}\text { Width } \\
\text { [min] }\end{array}$ & $\begin{array}{r}\text { Area } \\
{\left[\mathrm{pA}^{\star} \mathrm{s}\right]}\end{array}$ & $\begin{array}{l}\text { Height } \\
{[\mathrm{pA}]}\end{array}$ & $\begin{array}{c}\text { Area } \\
\frac{\circ}{0}\end{array}$ \\
\hline & ----- & & -- & --- & ----- & -- \\
\hline 1 & 60.847 & $\mathrm{MF}$ & 0.1125 & 34.14846 & 5.06002 & 0260 \\
\hline 2 & 61.548 & FM & 0.1064 & 33.73762 & 5.28506 & 49.69740 \\
\hline
\end{tabular}


$\beta$-Cyclosil, $40 \rightarrow 200{ }^{\circ} \mathrm{C}, 1.5^{\circ} / \mathrm{min}, 14 \mathrm{psi}$

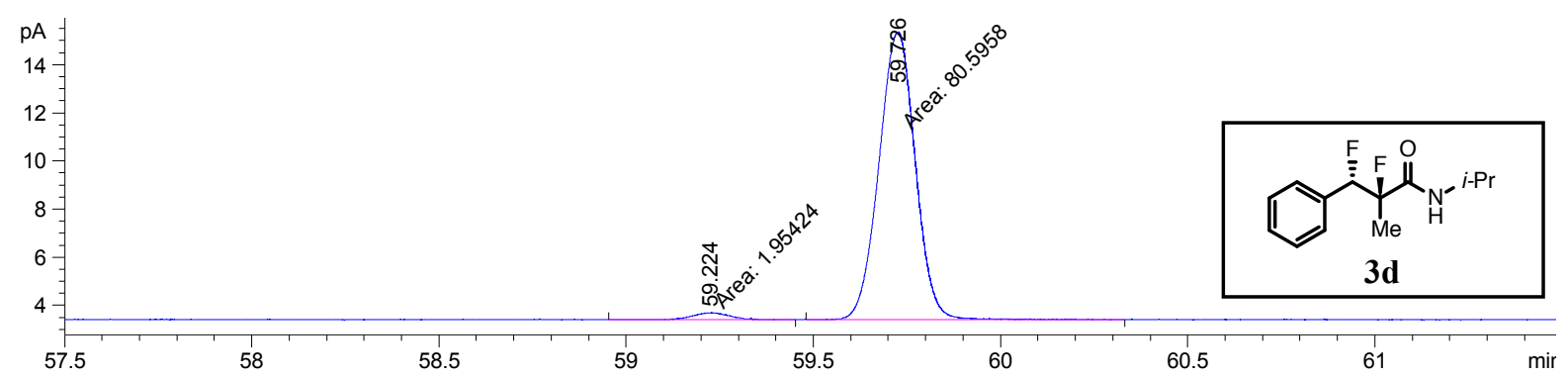

\begin{tabular}{|c|c|c|c|c|c|c|}
\hline $\begin{array}{c}\text { Peak } \\
\quad \#\end{array}$ & $\begin{array}{c}\text { RetTime } \\
\text { [min] }\end{array}$ & Type & $\begin{array}{l}\text { Width } \\
\text { [min] }\end{array}$ & $\begin{array}{r}\text { Area } \\
{\left[\mathrm{pA}^{\star} \mathrm{s}\right]}\end{array}$ & $\begin{array}{l}\text { Height } \\
{[\mathrm{pA}]}\end{array}$ & $\begin{array}{c}\text { Area } \\
\quad \frac{\circ}{0}\end{array}$ \\
\hline \multicolumn{7}{|c|}{$--|-------|----||-------||-----------|----------| \mid--------$} \\
\hline 1 & 59.224 & $\mathrm{MF}$ & 0.1123 & 1.95424 & $2.89971 e-1$ & 36734 \\
\hline 2 & 59.726 & FM & 0.1124 & 80.59579 & 11.94595 & 97.63266 \\
\hline
\end{tabular}

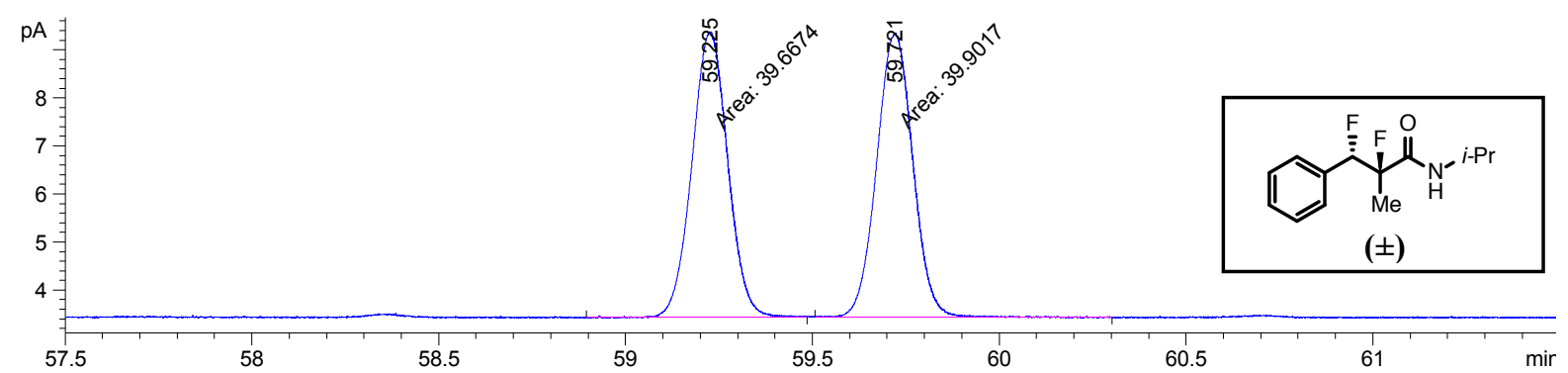

$\begin{array}{cccccc}\begin{array}{c}\text { Peak RetTime Type } \\ \text { Width } \\ \text { [min] }\end{array} & \begin{array}{c}\text { Area } \\ {[\mathrm{min}]}\end{array} & \begin{array}{c}\text { Height } \\ {\left[\mathrm{pA}^{\star} \mathrm{S}\right]}\end{array} & \begin{array}{c}\text { Area } \\ {[\mathrm{pA}]}\end{array} & \% \\ ----|-------| & ----\mid & -------\mid & ---------\mid & ---------|-------| \\ \quad 1 & 59.225 \mathrm{MF} & 0.1112 & 39.66742 & 5.94299 & 49.85278 \\ 2 & 59.721 \mathrm{FM} & 0.1124 & 39.90170 & 5.91511 & 50.14722\end{array}$


CP-Chirasil-Dex CB, $120{ }^{\circ} \mathrm{C}, 14 \mathrm{psi}$

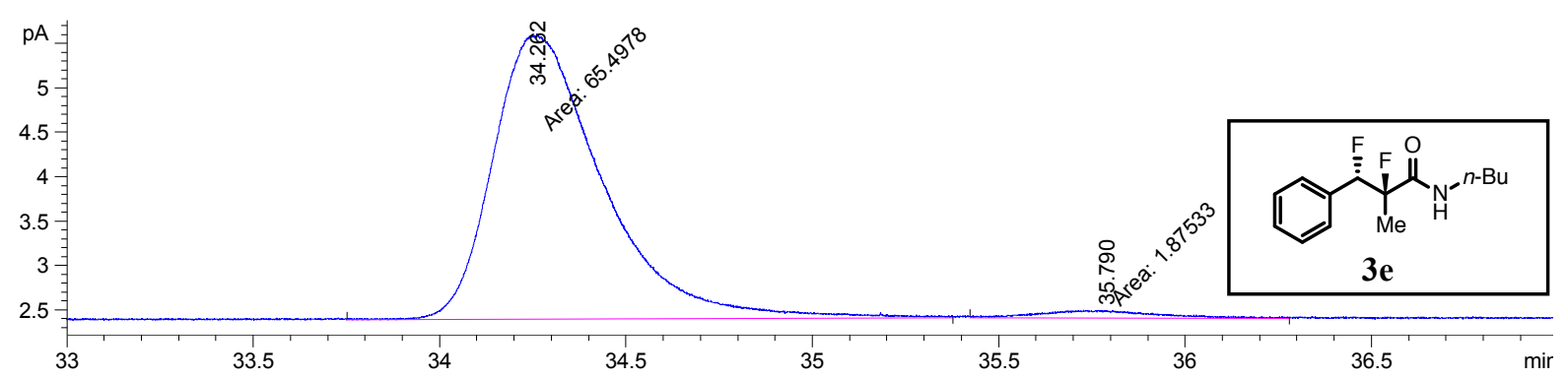

$\begin{array}{cccccc}\begin{array}{c}\text { Peak RetTime Type } \\ \text { \# }\end{array} \quad \begin{array}{c}\text { Width } \\ {[\mathrm{min}]}\end{array} & \begin{array}{c}\text { Area } \\ {\left[\mathrm{pA}^{\star} \mathrm{s}\right]}\end{array} & \begin{array}{c}\text { Height } \\ {[\mathrm{pA}]}\end{array} & \begin{array}{c}\text { Area } \\ \%\end{array} \\ -1 & 34.262 \mathrm{MM} & 0.3416 & 65.49781 & 3.19604 & 97.21650 \\ 2 & 35.790 \mathrm{MM} & 0.3603 & 1.87533 & 8.67437 e-2 & 2.78350\end{array}$

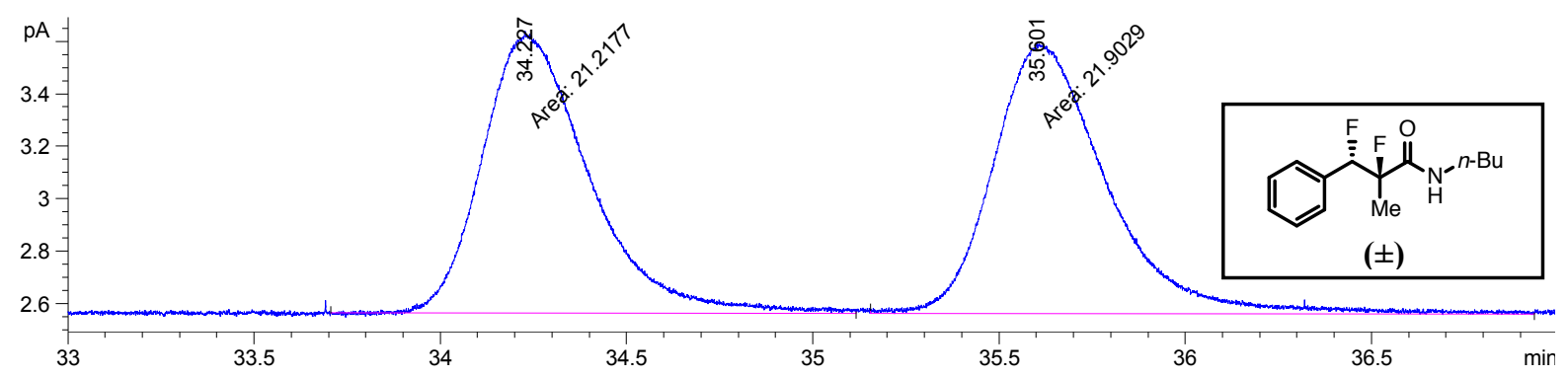

\begin{tabular}{|c|c|c|c|c|c|c|}
\hline $\begin{array}{c}\text { Peak } \\
\quad \#\end{array}$ & $\begin{array}{c}\text { RetTime } \\
\text { [min] }\end{array}$ & Type & $\begin{array}{l}\text { Width } \\
\text { [min] }\end{array}$ & $\begin{array}{r}\text { Area } \\
{\left[\mathrm{pA}^{\star} \mathrm{s}\right]}\end{array}$ & $\begin{array}{l}\text { Height } \\
{[\mathrm{pA}]}\end{array}$ & $\begin{array}{c}\text { Area } \\
\frac{\circ}{0}\end{array}$ \\
\hline & & & & -- & -1 & --- \\
\hline 1 & 34.227 & $\mathrm{MF}$ & 0.3321 & 21.21774 & 1.06498 & 558 \\
\hline 2 & 35.601 & FM & 0.3547 & 21.90285 & 1.02917 & 50.79442 \\
\hline
\end{tabular}


$\beta$-Cyclosil, $40 \rightarrow 200{ }^{\circ} \mathrm{C}, 1.5^{\circ} / \mathrm{min}, 14 \mathrm{psi}$
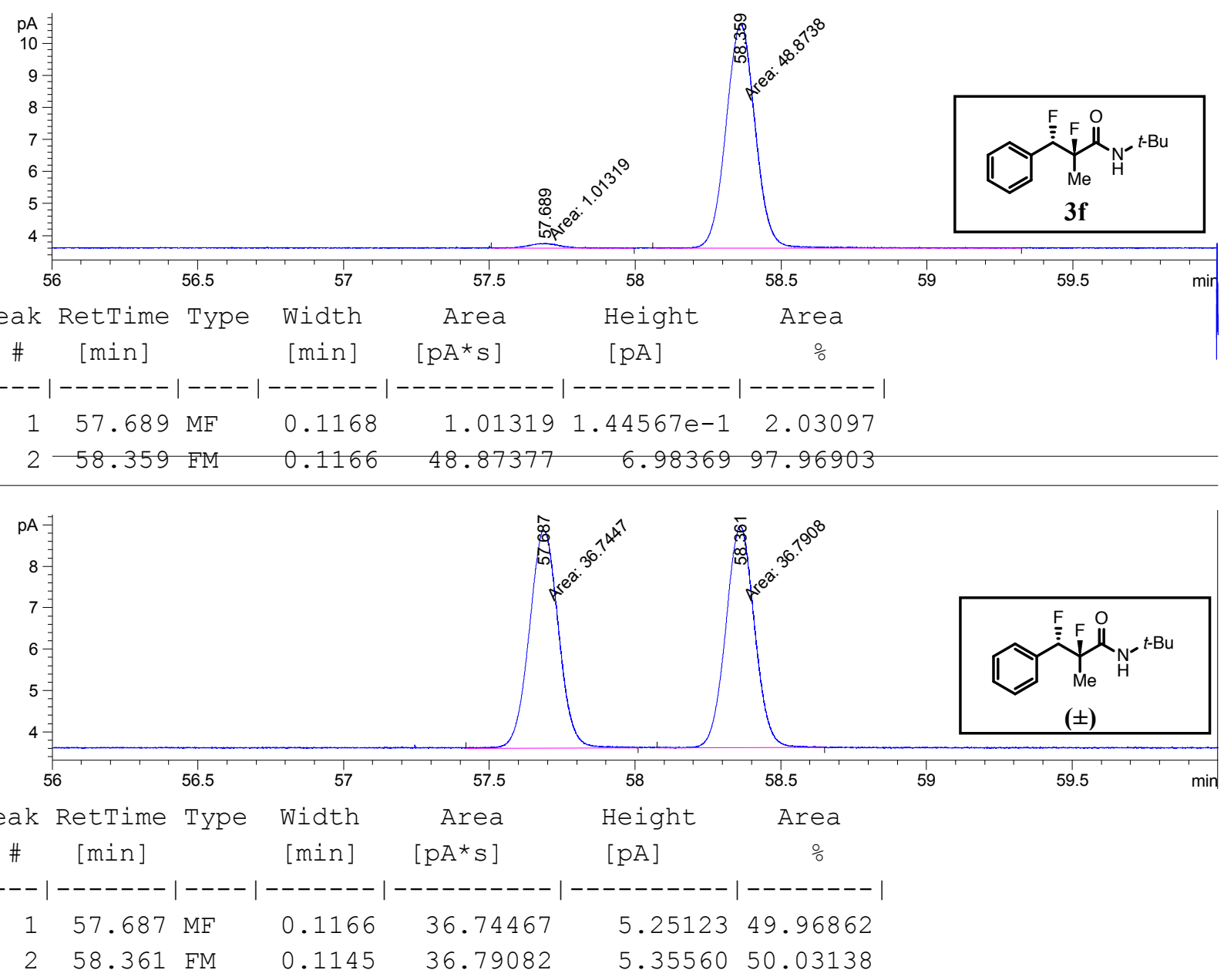
AD-H, $1 \mathrm{~mL} / \mathrm{min}, 2 \% \mathrm{IPA} /$ hexanes, $220 \mathrm{~nm}$

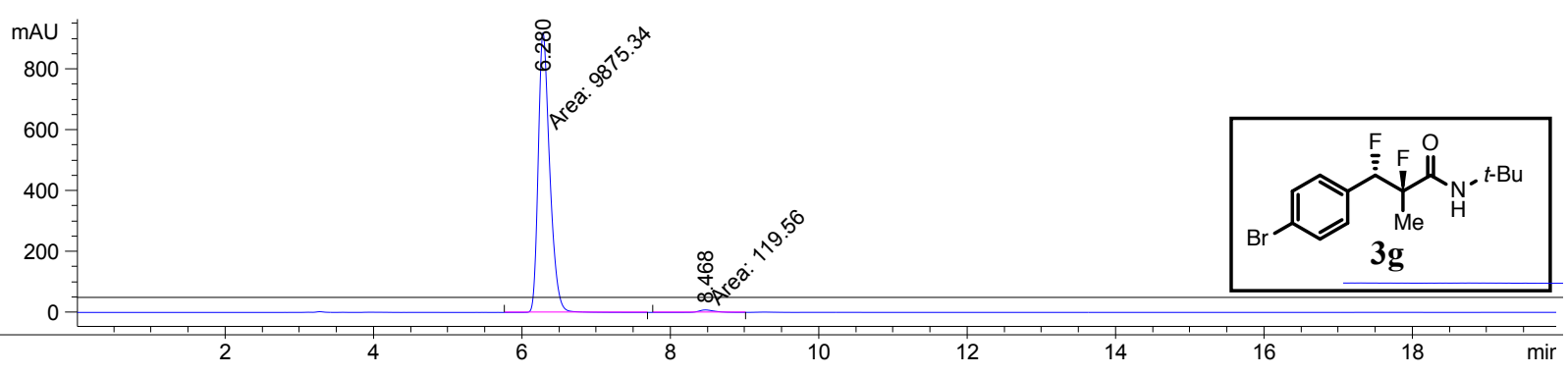

\begin{tabular}{|c|c|c|c|c|c|c|}
\hline $\begin{array}{c}\text { Peak } \\
\#\end{array}$ & $\begin{array}{c}\text { RetTime } \\
\text { [min] }\end{array}$ & Type & $\begin{array}{c}\text { Width } \\
\text { [min] }\end{array}$ & $\begin{array}{c}\text { Area } \\
{\left[m A U^{\star} s\right]}\end{array}$ & $\begin{array}{l}\text { Height } \\
{[\mathrm{mAU}]}\end{array}$ & $\begin{array}{c}\text { Area } \\
\frac{\circ}{0}\end{array}$ \\
\hline & 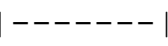 & & 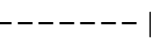 & ---- & -------- & $-------\mid$ \\
\hline 1 & 6.280 & MF & 0.1789 & 9875.33594 & 919.87378 & 98.8038 \\
\hline 2 & 8.468 & FM & 0.2477 & 119.56028 & 8.04568 & 1.1962 \\
\hline
\end{tabular}

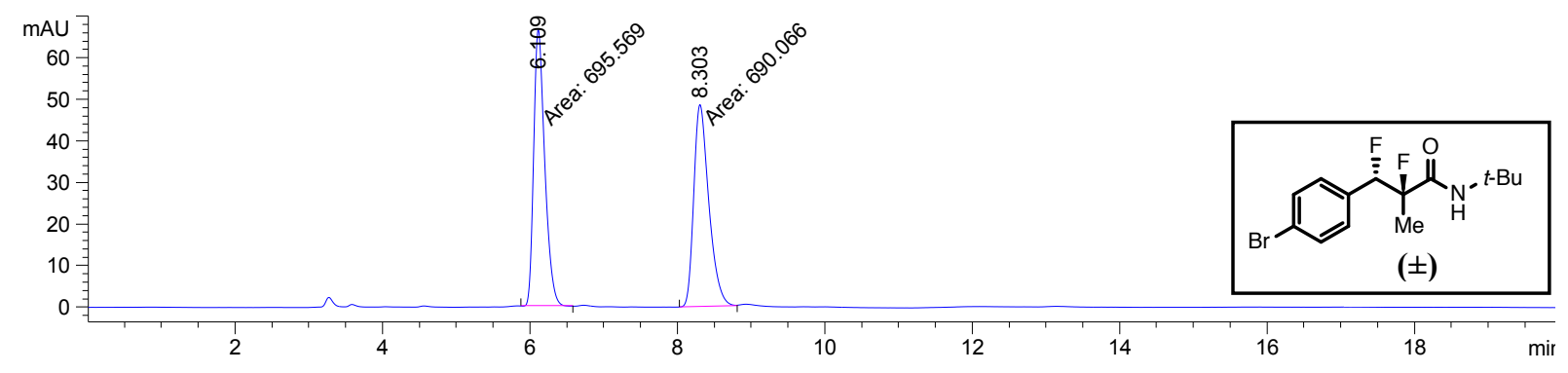

\begin{tabular}{|c|c|c|c|c|c|c|}
\hline $\begin{array}{c}\text { Peak } \\
\quad \#\end{array}$ & $\begin{array}{c}\text { RetTime } \\
\text { [min] }\end{array}$ & Type & $\begin{array}{l}\text { Width } \\
\text { [min] }\end{array}$ & $\begin{array}{c}\text { Area } \\
{\left[m A U^{\star} s\right]}\end{array}$ & $\begin{array}{l}\text { Height } \\
{[\mathrm{mAU}]}\end{array}$ & $\begin{array}{c}\text { Area } \\
\frac{\circ}{0}\end{array}$ \\
\hline & & & & & & \\
\hline 1 & 6.109 & $\mathrm{MM}$ & 0.1742 & 695.56879 & 66.54630 & 986 \\
\hline 2 & 8.303 & $\mathrm{MM}$ & 0.2366 & 690.06641 & 48.61501 & 49.8014 \\
\hline
\end{tabular}


$\beta$-Cyclosil, $40 \rightarrow 200{ }^{\circ} \mathrm{C}, 0.2^{\circ} / \mathrm{min}, 14 \mathrm{psi}$
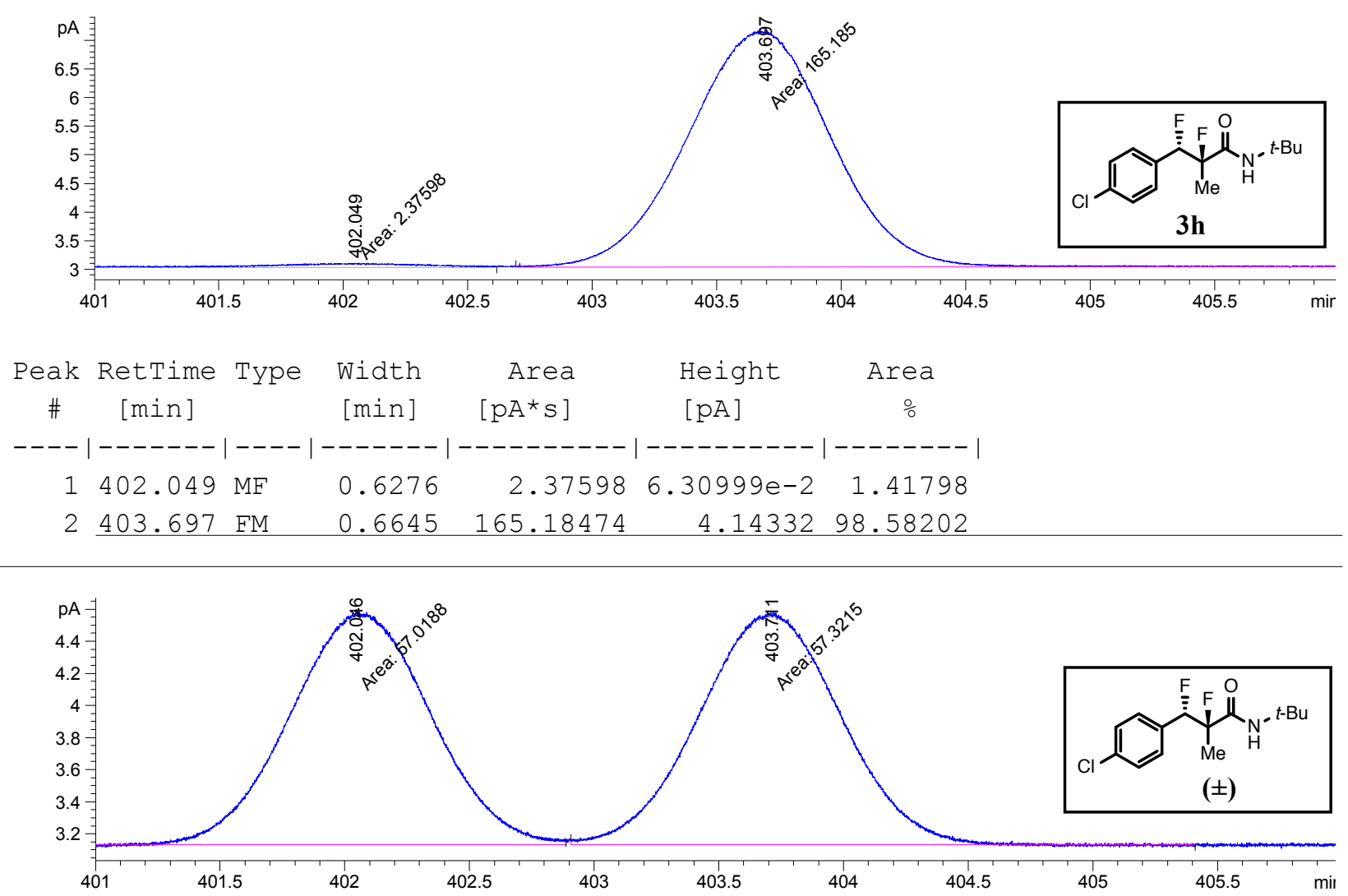

\begin{tabular}{|c|c|c|c|c|c|c|}
\hline $\begin{array}{c}\text { Peak } \\
\quad \#\end{array}$ & $\begin{array}{c}\text { RetTime } \\
\text { [min] }\end{array}$ & Type & $\begin{array}{l}\text { Width } \\
\text { [min] }\end{array}$ & $\begin{array}{r}\text { Area } \\
{\left[\mathrm{pA}^{*} \mathrm{~S}\right]}\end{array}$ & $\begin{array}{l}\text { Height } \\
{[p A]}\end{array}$ & $\begin{array}{c}\text { Area } \\
\frac{\circ}{0}\end{array}$ \\
\hline 1 & 402 & $\mathrm{MF}$ & 6522 & 57.01876 & 5698 & 49.86762 \\
\hline 2 & 403.711 & FM & .6590 & 57.32150 & 1.44978 & 50.13238 \\
\hline
\end{tabular}


$\beta$-Cyclosil, $40 \rightarrow 200{ }^{\circ} \mathrm{C}, 1.5^{\circ} / \mathrm{min}, 14 \mathrm{psi}$

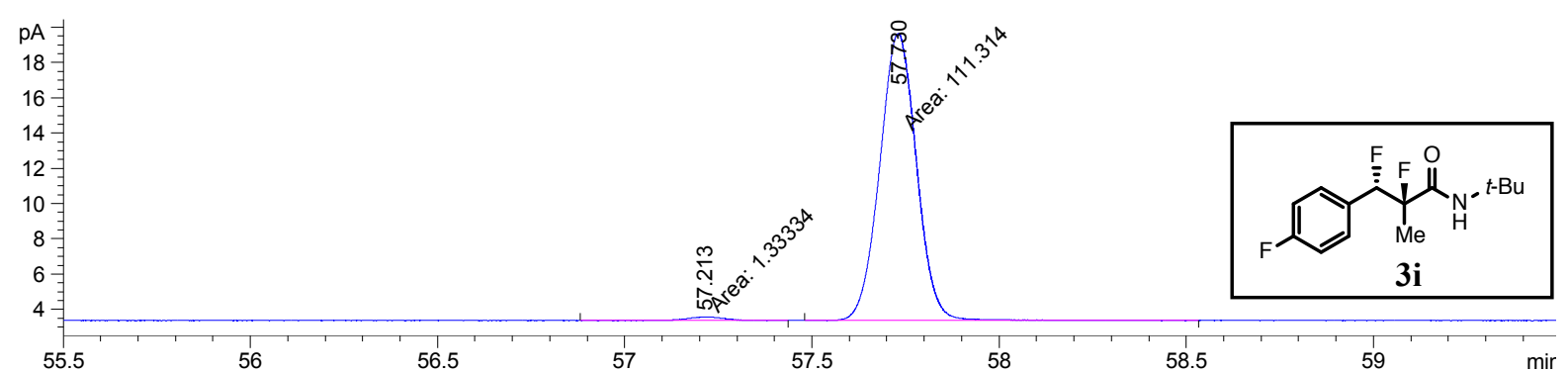

\begin{tabular}{|c|c|c|c|c|c|c|}
\hline $\begin{array}{c}\text { Peak } \\
\quad \#\end{array}$ & $\begin{array}{c}\text { RetTime } \\
\text { [min] }\end{array}$ & Type & $\begin{array}{l}\text { Width } \\
\text { [min] }\end{array}$ & $\begin{array}{r}\text { Area } \\
{\left[\mathrm{pA}^{\star} \mathrm{s}\right]}\end{array}$ & $\begin{array}{l}\text { Height } \\
{[\mathrm{pA}]}\end{array}$ & $\begin{array}{c}\text { Area } \\
\frac{\circ}{0}\end{array}$ \\
\hline & & & -- & 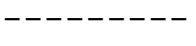 & $=-1$ - & ---- \\
\hline 1 & 57.213 & MF & 0.1074 & 1.33334 & $2.06846 e-1$ & 1.18364 \\
\hline 2 & 57.730 & FM & 0.1139 & 111.31385 & 16.28446 & 98.81636 \\
\hline
\end{tabular}

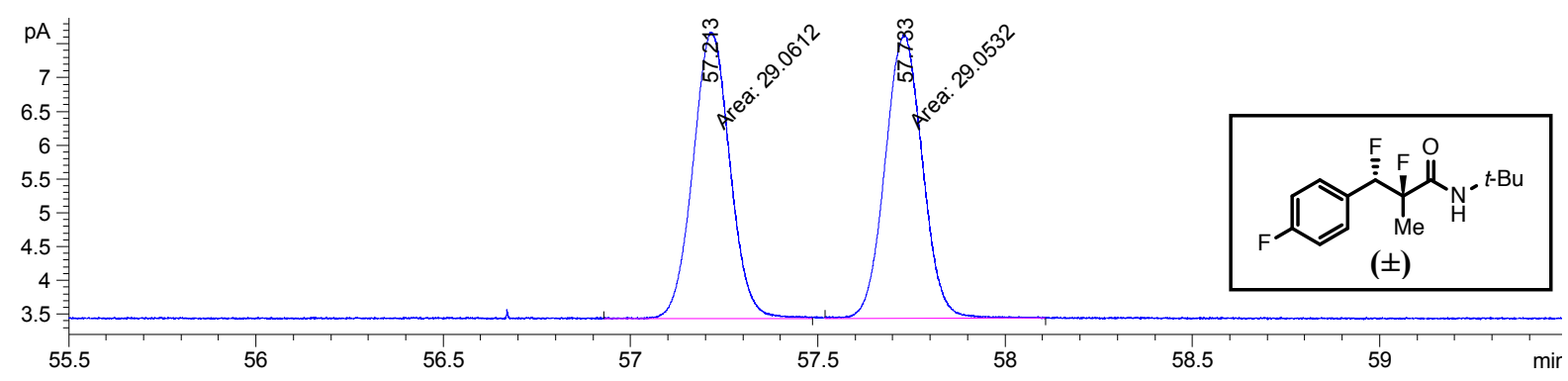

$\begin{array}{cccccc}\begin{array}{c}\text { Peak RetTime Type Width } \\ \text { \# }[\mathrm{min}]\end{array} & \begin{array}{c}\text { Area } \\ {[\mathrm{min}]}\end{array} & \begin{array}{c}\text { Height } \\ {\left[\mathrm{pA}^{*} \mathrm{~S}\right]}\end{array} & \begin{array}{c}\text { Area } \\ {[\mathrm{pA}]}\end{array} & \frac{\circ}{0} \\ ----|-------|----\mid & -------\mid & --------1 & ---------|-------| \\ 1 & 57.213 \mathrm{MF} & 0.1144 & 29.06118 & 4.23258 & 50.00688 \\ 2 & 57.733 \mathrm{FM} & 0.1158 & 29.05319 & 4.18074 & 49.99312\end{array}$


AD-H, $1 \mathrm{~mL} / \mathrm{min}, 2 \%$ IPA/hexanes, $220 \mathrm{~nm}$

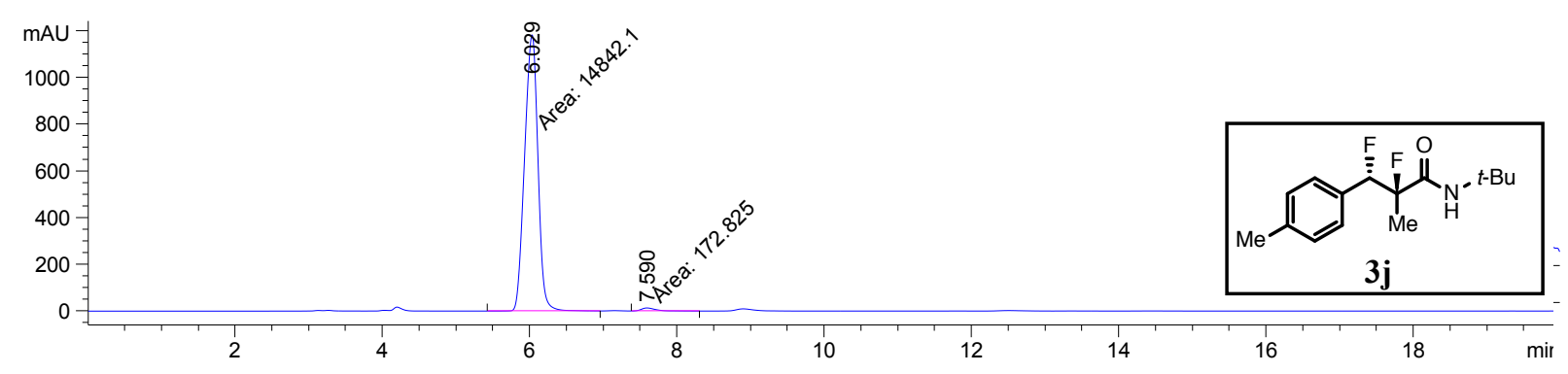

\begin{tabular}{|c|c|c|c|c|c|c|}
\hline $\begin{array}{c}\text { Peak } \\
\quad \#\end{array}$ & $\begin{array}{c}\text { RetTime } \\
\text { [min] }\end{array}$ & Type & $\begin{array}{l}\text { Width } \\
\text { [min] }\end{array}$ & $\begin{array}{c}\text { Area } \\
{\left[m A U^{\star} \mathrm{s}\right]}\end{array}$ & $\begin{array}{l}\text { Height } \\
{[\mathrm{mAU}]}\end{array}$ & $\begin{array}{c}\text { Area } \\
\frac{\circ}{0}\end{array}$ \\
\hline & & & & . & . & \\
\hline 1 & 6.029 & $\mathrm{MM}$ & 0.2090 & $1.48421 e 4$ & 1183.62939 & 98.8490 \\
\hline 2 & 7.590 & MM & 0.2217 & 172.82478 & 12.99449 & 1.1510 \\
\hline
\end{tabular}

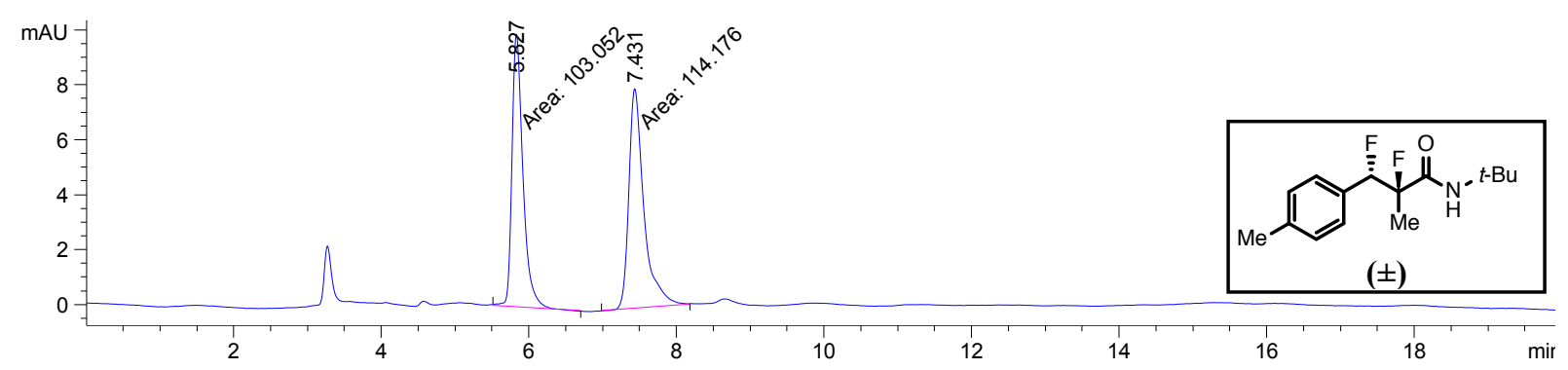

\begin{tabular}{|c|c|c|c|c|c|c|}
\hline $\begin{array}{c}\text { Peak } \\
\text { \# }\end{array}$ & $\begin{array}{c}\text { RetTime } \\
\text { [min] }\end{array}$ & Type & $\begin{array}{c}\text { Width } \\
\text { [min] }\end{array}$ & $\begin{array}{c}\text { Area } \\
{\left[\mathrm{mAU}^{*} \mathrm{~s}\right]}\end{array}$ & $\begin{array}{l}\text { Height } \\
\text { [mAU] }\end{array}$ & $\begin{array}{c}\text { Area } \\
\frac{\circ}{0}\end{array}$ \\
\hline-1 & ------ & & . & -----1 & ------- & ---1 \\
\hline 1 & 5.827 & MM & 0.1728 & 103.05161 & 9.94025 & 4396 \\
\hline 2 & 7.431 & MM & 0.2378 & 114.17554 & 8.00156 & 52.5604 \\
\hline
\end{tabular}


$\beta$-Cyclosil, $40 \rightarrow 200{ }^{\circ} \mathrm{C}, 1.5^{\circ} / \mathrm{min}, 14 \mathrm{psi}$
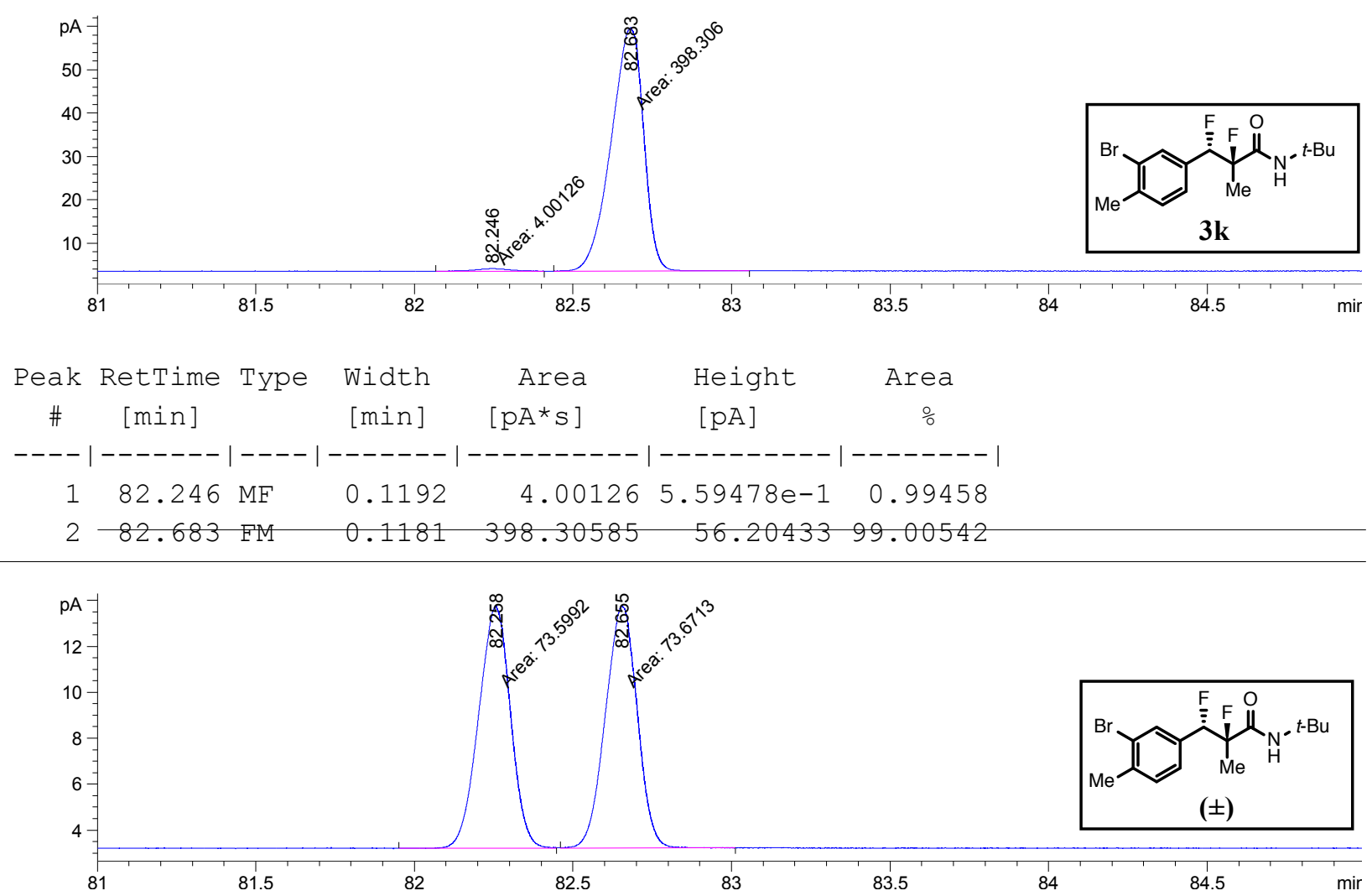

\begin{tabular}{|c|c|c|c|c|c|c|}
\hline $\begin{array}{c}\text { Peak } \\
\quad \#\end{array}$ & $\begin{array}{c}\text { RetTime } \\
\text { [min] }\end{array}$ & Type & $\begin{array}{l}\text { Width } \\
\text { [min] }\end{array}$ & $\begin{array}{r}\text { Area } \\
{\left[\mathrm{pA}^{\star} \mathrm{s}\right]}\end{array}$ & $\begin{array}{l}\text { Height } \\
{[\mathrm{pA}]}\end{array}$ & $\begin{array}{c}\text { Area } \\
\frac{\circ}{0}\end{array}$ \\
\hline 1 & 82 & $\mathrm{MF}$ & 61 & 9922 & 6298 & 551 \\
\hline 2 & 82.655 & FM & 0.1164 & 73.67134 & 10.55102 & 50.02449 \\
\hline
\end{tabular}


$\beta$-Cyclosil, $40 \rightarrow 200{ }^{\circ} \mathrm{C}, 1.5^{\circ} / \mathrm{min}, 14 \mathrm{psi}$
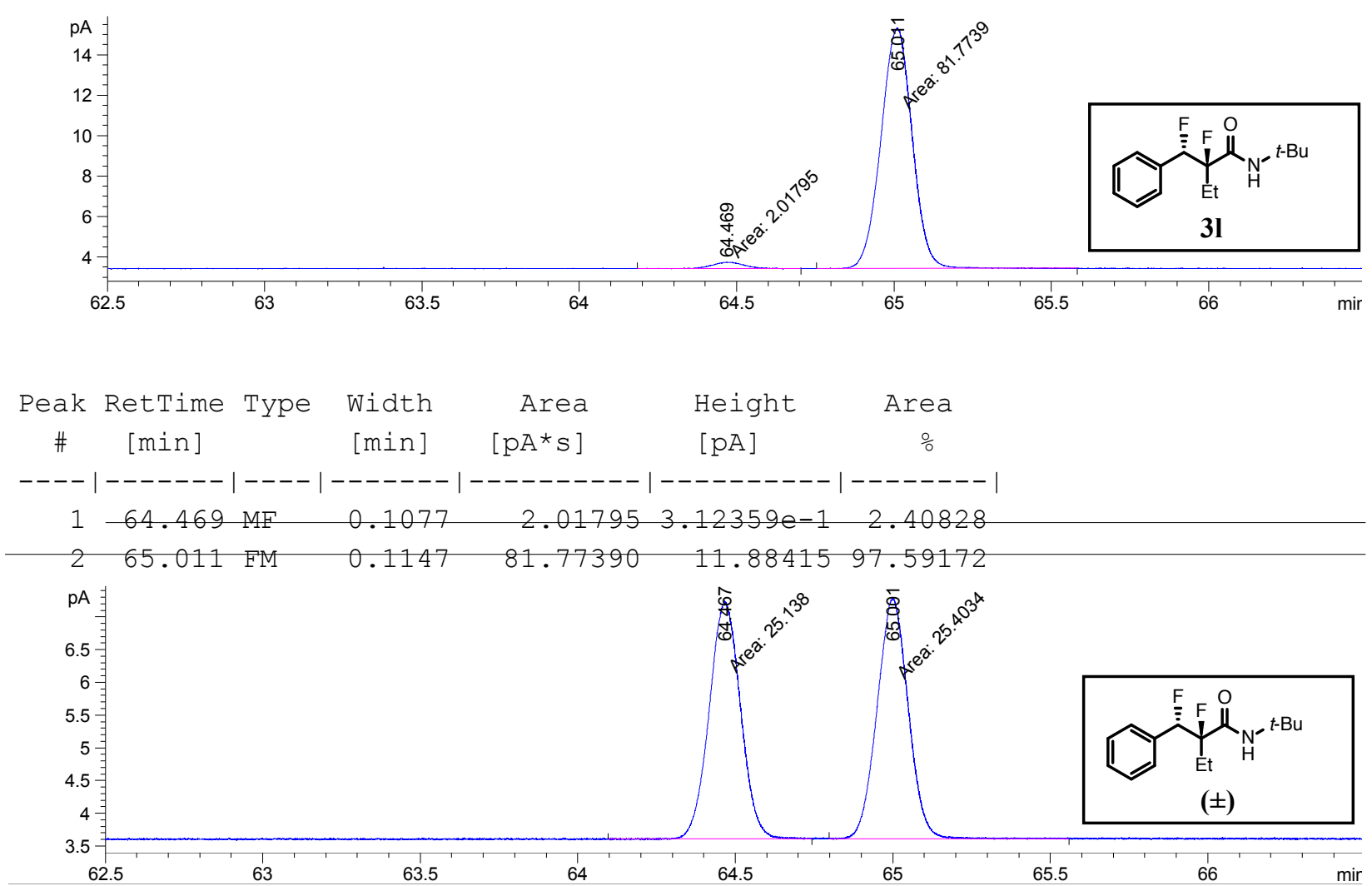

\begin{tabular}{|c|c|c|c|c|c|c|}
\hline $\begin{array}{c}\text { Peak } \\
\#\end{array}$ & $\begin{array}{c}\text { RetTime } \\
\text { [min] }\end{array}$ & Type & $\begin{array}{l}\text { Width } \\
\text { [min] }\end{array}$ & $\begin{array}{r}\text { Area } \\
{\left[\mathrm{pA}^{\star} \mathrm{s}\right]}\end{array}$ & $\begin{array}{l}\text { Height } \\
{[\mathrm{pA}]}\end{array}$ & $\begin{array}{c}\text { Area } \\
\quad \%\end{array}$ \\
\hline 1 & 64.467 & $\mathrm{MF}$ & 0.1156 & 25.13804 & 3.62285 & 3749 \\
\hline 2 & 65.001 & FM & 0.1150 & 25.40339 & 3.68101 & 50.26251 \\
\hline
\end{tabular}


$\beta$-Cyclosil, $40 \rightarrow 200{ }^{\circ} \mathrm{C}, 1.5^{\circ} / \mathrm{min}, 14 \mathrm{psi}$

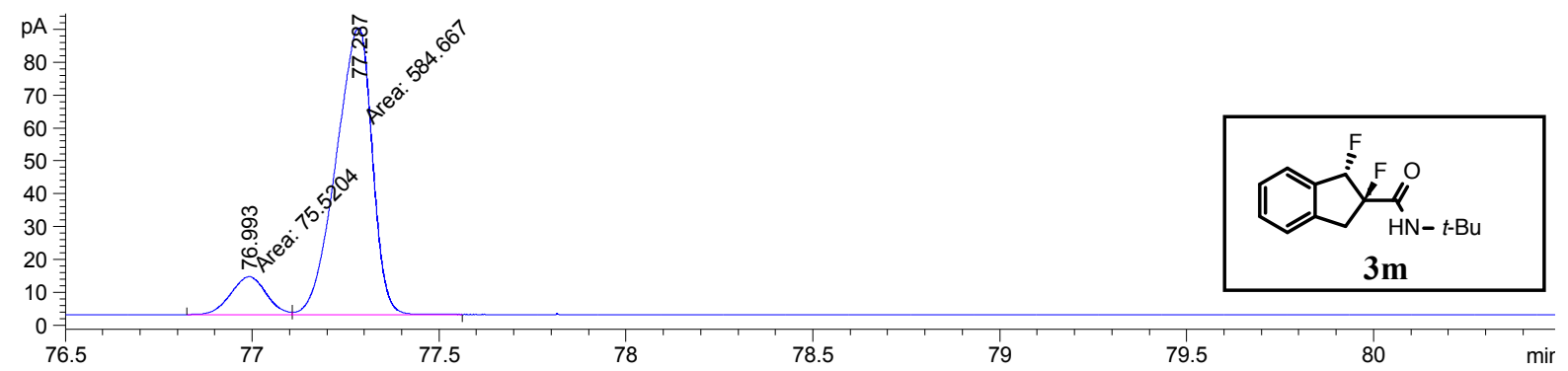

\begin{tabular}{|c|c|c|c|c|c|c|}
\hline $\begin{array}{c}\text { Peak } \\
\#\end{array}$ & $\begin{array}{c}\text { RetTime } \\
\text { [min] }\end{array}$ & Type & $\begin{array}{l}\text { Width } \\
\text { [min] }\end{array}$ & $\begin{array}{r}\text { Area } \\
{\left[\mathrm{pA}^{\star} \mathrm{s}\right]}\end{array}$ & $\begin{array}{l}\text { Height } \\
{[\mathrm{pA}]}\end{array}$ & $\begin{array}{c}\text { Area } \\
\frac{\circ}{0}\end{array}$ \\
\hline 1 & 76.993 & $\mathrm{MF}$ & 088 & 2040 & & 3923 \\
\hline 2 & 77.287 & FM & 0.1118 & 584.66730 & 87.17343 & 88.56077 \\
\hline
\end{tabular}

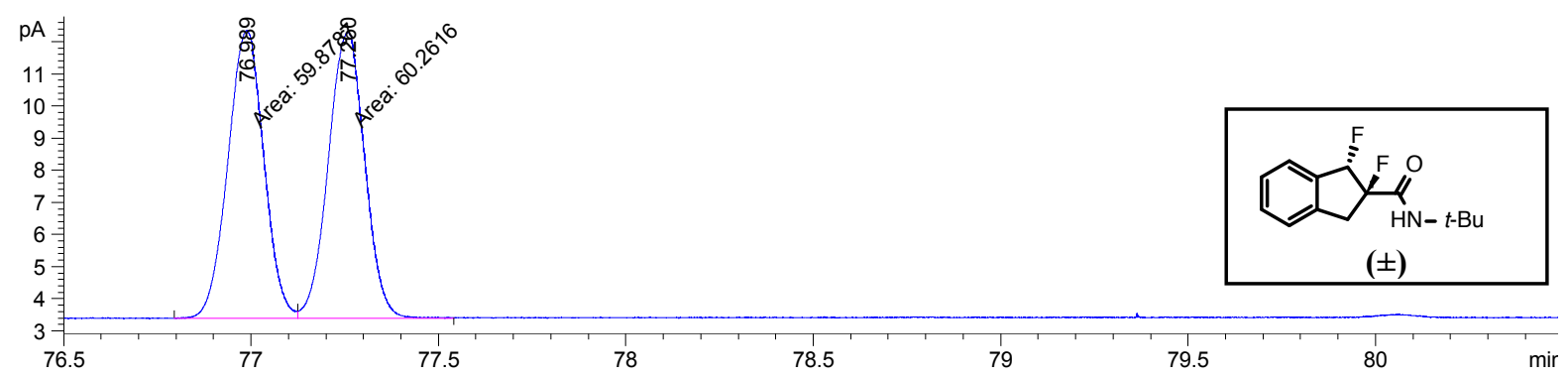

$\begin{array}{cccccc}\begin{array}{c}\text { Peak RetTime Type Width } \\ \text { \# }[\mathrm{min}]\end{array} & \begin{array}{c}\text { Area } \\ {[\mathrm{min}]}\end{array} & \begin{array}{c}\text { Height } \\ {\left[\mathrm{pA}^{*} \mathrm{~S}\right]}\end{array} & \begin{array}{c}\text { Area } \\ {[\mathrm{pA}]}\end{array} & \frac{\circ}{0} \\ ----|-------|----\mid & -------\mid & --------1 & ---------|-------| \\ 1 & 76.989 \mathrm{MF} & 0.1114 & 59.87873 & 8.95794 & 49.84067 \\ 2 & 77.260 \mathrm{FM} & 0.1130 & 60.26156 & 8.88825 & 50.15933\end{array}$


CP-Chirasil-Dex CB, $40 \rightarrow 160{ }^{\circ} \mathrm{C}, 1.5^{\circ} / \mathrm{min}, 14 \mathrm{psi}$

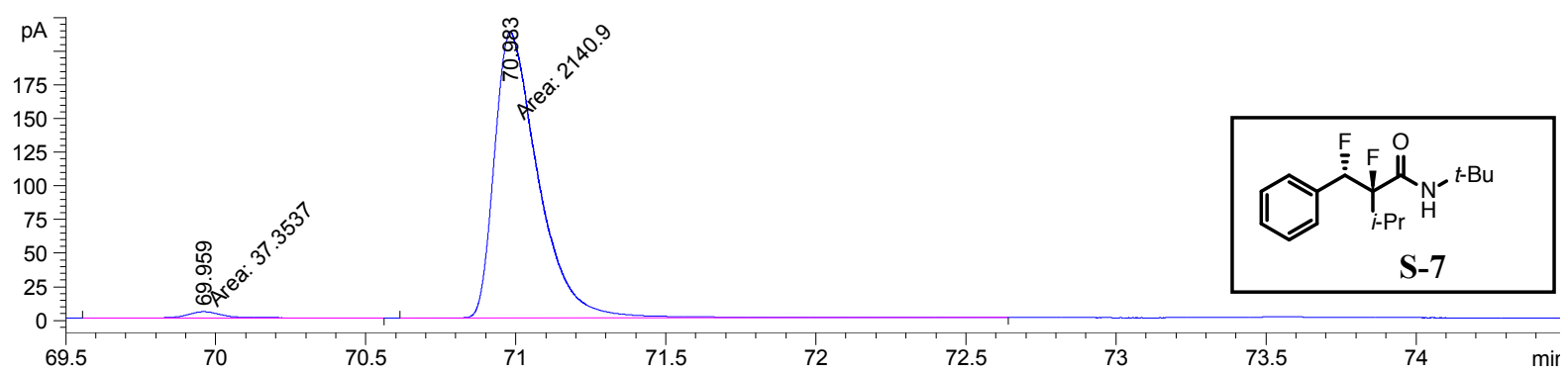

\begin{tabular}{|c|c|c|c|c|c|c|}
\hline $\begin{array}{c}\text { Peak } \\
\quad \#\end{array}$ & $\begin{array}{c}\text { RetTime } \\
\text { [min] }\end{array}$ & Type & $\begin{array}{c}\text { Width } \\
\text { [min] }\end{array}$ & $\begin{array}{r}\text { Area } \\
{\left[\mathrm{pA}^{*} \mathrm{~s}\right]}\end{array}$ & $\begin{array}{l}\text { Height } \\
{[\mathrm{pA}]}\end{array}$ & $\begin{array}{c}\text { Area } \\
\frac{\circ}{0}\end{array}$ \\
\hline & --1 & & & -------- & --- & \\
\hline 1 & 69.959 & $\mathrm{MF}$ & 0.1346 & 37.35367 & 4.62651 & 1485 \\
\hline 2 & 70.983 & FM & 0.1674 & 2140.89941 & 213.09407 & 98.28515 \\
\hline
\end{tabular}

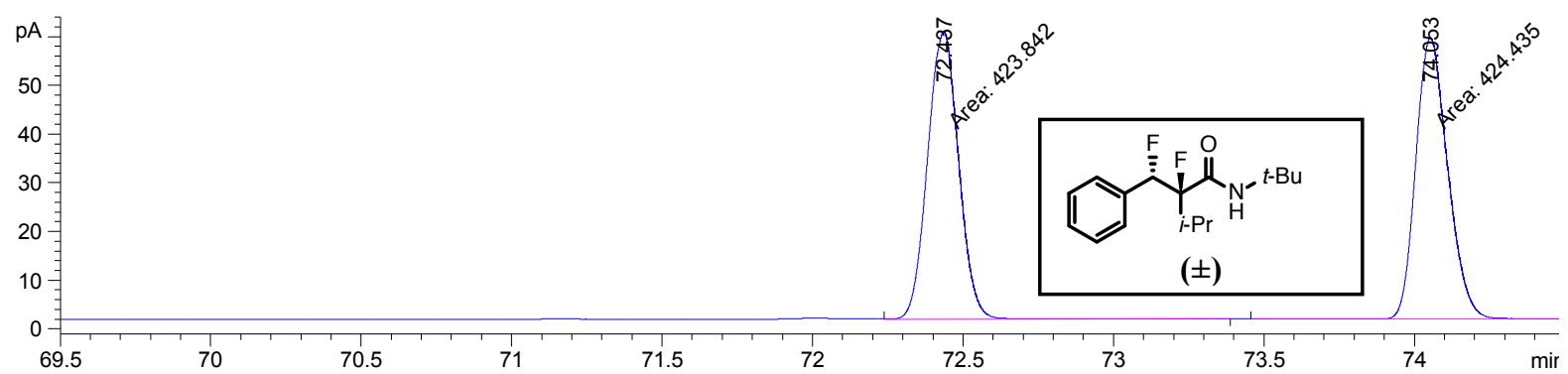

\begin{tabular}{|c|c|c|c|c|c|c|}
\hline $\begin{array}{c}\text { Peak } \\
\#\end{array}$ & $\begin{array}{c}\text { RetTime } \\
\text { [min] }\end{array}$ & Type & $\begin{array}{l}\text { Width } \\
\text { [min] }\end{array}$ & $\begin{array}{r}\text { Area } \\
{\left[\mathrm{pA}^{*} \mathrm{~S}\right]}\end{array}$ & $\begin{array}{l}\text { Height } \\
{[\mathrm{pA}]}\end{array}$ & $\begin{array}{c}\text { Area } \\
\quad \circ\end{array}$ \\
\hline & & & & & & 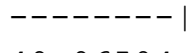 \\
\hline 1 & 72.437 & $\mathrm{MF}$ & 0.1195 & 423.84232 & 59.11995 & 6504 \\
\hline 2 & 74.053 & FM & 0.1221 & 424.43536 & 57.91782 & 50.03496 \\
\hline
\end{tabular}


$\beta$-Cyclosil, $40 \rightarrow 200{ }^{\circ} \mathrm{C}, 1.5^{\circ} / \mathrm{min}, 14 \mathrm{psi}$
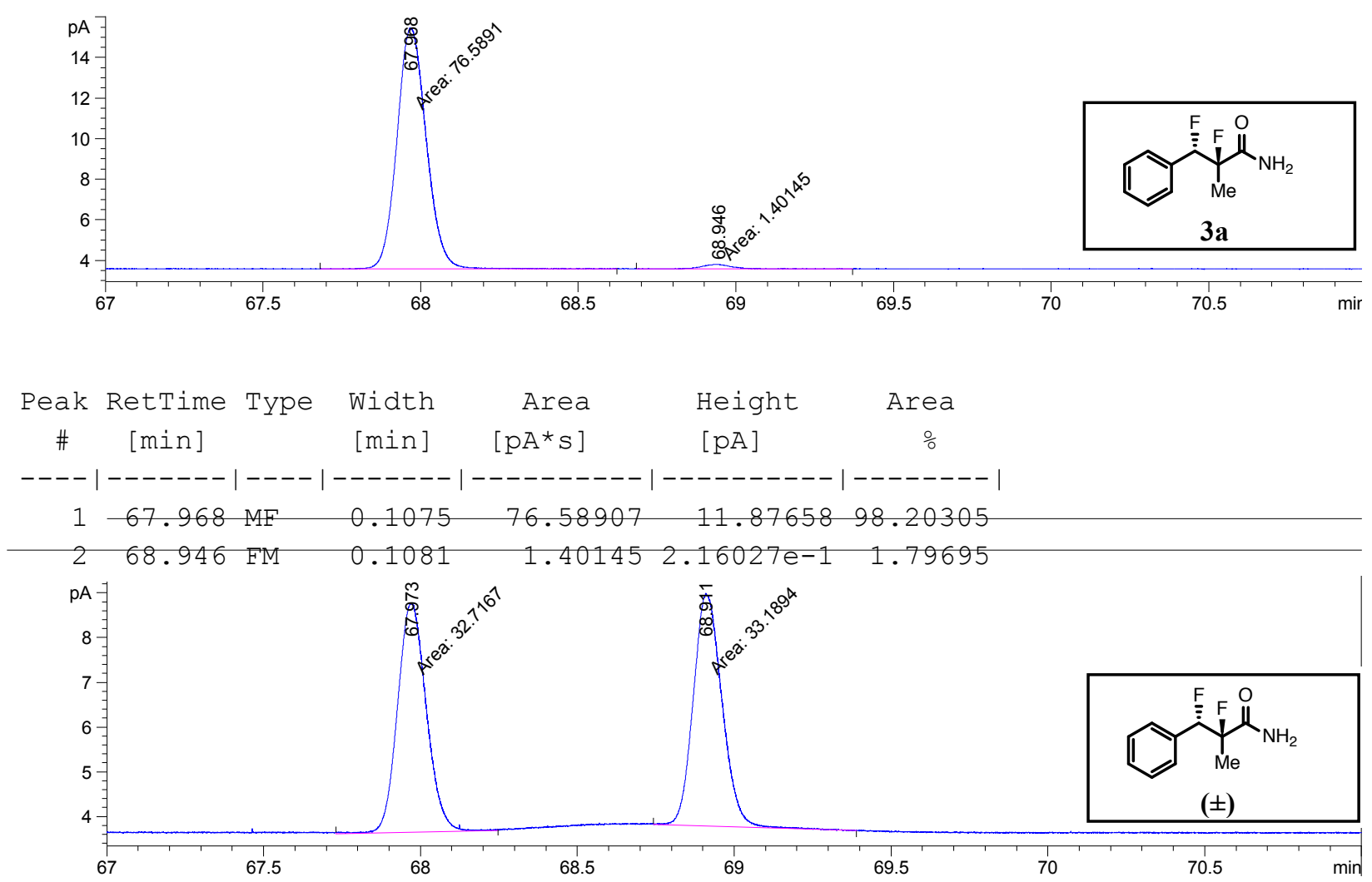

\begin{tabular}{|c|c|c|c|c|c|c|}
\hline $\begin{array}{c}\text { Peak } \\
\quad \#\end{array}$ & $\begin{array}{c}\text { RetTime } \\
\text { [min] }\end{array}$ & Type & $\begin{array}{l}\text { Width } \\
\text { [min] }\end{array}$ & $\begin{array}{r}\text { Area } \\
{\left[\mathrm{pA}{ }^{*} \mathrm{~s}\right]}\end{array}$ & $\begin{array}{l}\text { Height } \\
{[\mathrm{pA}]}\end{array}$ & $\begin{array}{c}\text { Area } \\
\quad \%\end{array}$ \\
\hline & & & & -------- & -------- & $|--------|$ \\
\hline 1 & 67.973 & $\mathrm{MM}$ & 0.1065 & 32.71674 & 5.12111 & 49.64138 \\
\hline 2 & 68.911 & $\mathrm{MM}$ & 0.1064 & 33.18945 & 5.19898 & 50.35862 \\
\hline
\end{tabular}


$\beta$-Cyclosil, $40 \rightarrow 200{ }^{\circ} \mathrm{C}, 1^{\circ} / \mathrm{min}, 20 \mathrm{psi}$

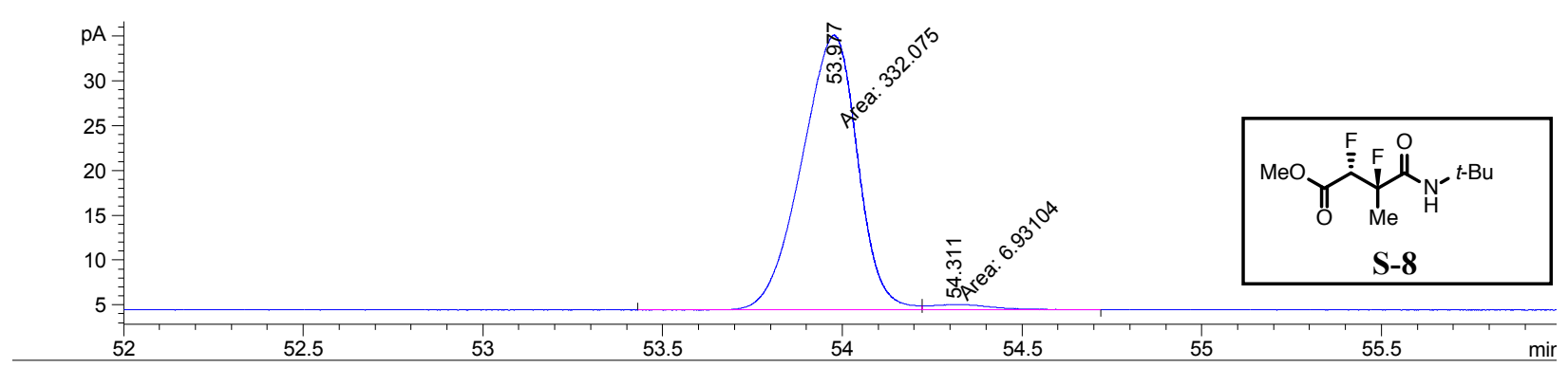

\begin{tabular}{|c|c|c|c|c|c|c|}
\hline $\begin{array}{c}\text { Peak } \\
\quad \#\end{array}$ & $\begin{array}{c}\text { RetTime } \\
\text { [min] }\end{array}$ & Type & $\begin{array}{l}\text { Width } \\
\text { [min] }\end{array}$ & $\begin{array}{r}\text { Area } \\
{\left[\mathrm{pA}^{\star} \mathrm{s}\right]}\end{array}$ & $\begin{array}{l}\text { Height } \\
{[\mathrm{pA}]}\end{array}$ & $\begin{array}{c}\text { Area } \\
\frac{\circ}{0}\end{array}$ \\
\hline & & MF & 30 & 547 & & 548 \\
\hline 2 & 54.311 & FM & 0.1952 & 6.93104 & $5.91823 e-1$ & 4452 \\
\hline
\end{tabular}

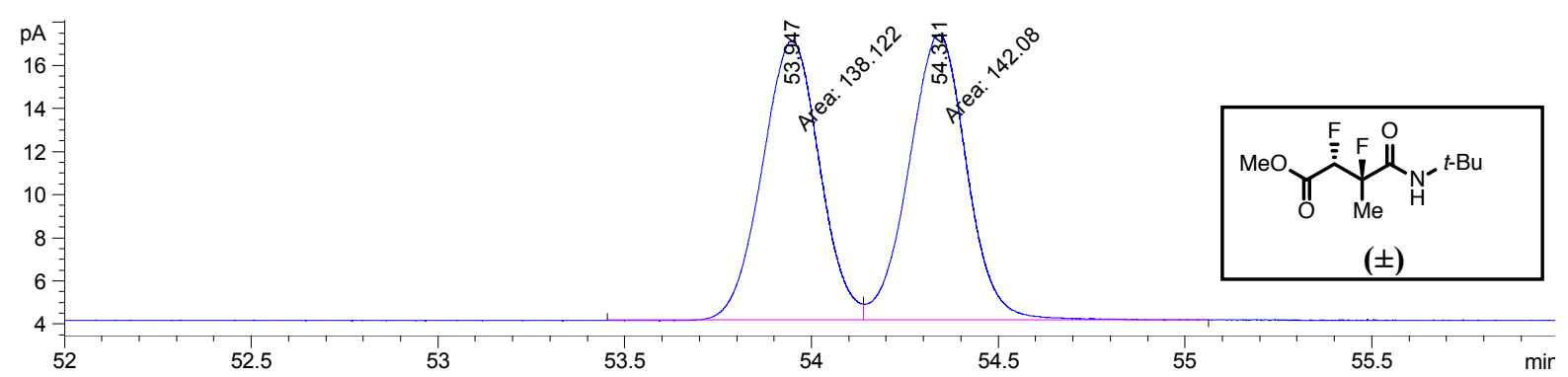

\begin{tabular}{|c|c|c|c|c|c|c|}
\hline $\begin{array}{c}\text { Peak } \\
\quad \#\end{array}$ & $\begin{array}{c}\text { RetTime } \\
\text { [min] }\end{array}$ & Type & $\begin{array}{l}\text { Width } \\
\text { [min] }\end{array}$ & $\begin{array}{r}\text { Area } \\
{\left[\mathrm{pA}^{*} \mathrm{~s}\right]}\end{array}$ & $\begin{array}{l}\text { Height } \\
{[\mathrm{pA}]}\end{array}$ & $\begin{array}{c}\text { Area } \\
\frac{\circ}{0}\end{array}$ \\
\hline 1 & 53.947 & $\mathrm{MF}$ & 0.1781 & 138.12201 & 398 & 365 \\
\hline 2 & 54.341 & FM & 0.1786 & 142.08043 & 13.25977 & 50.70635 \\
\hline
\end{tabular}


$\beta$-Cyclosil, $120 \rightarrow 200{ }^{\circ} \mathrm{C}, 1 \% \mathrm{~min}, 7 \mathrm{psi}$

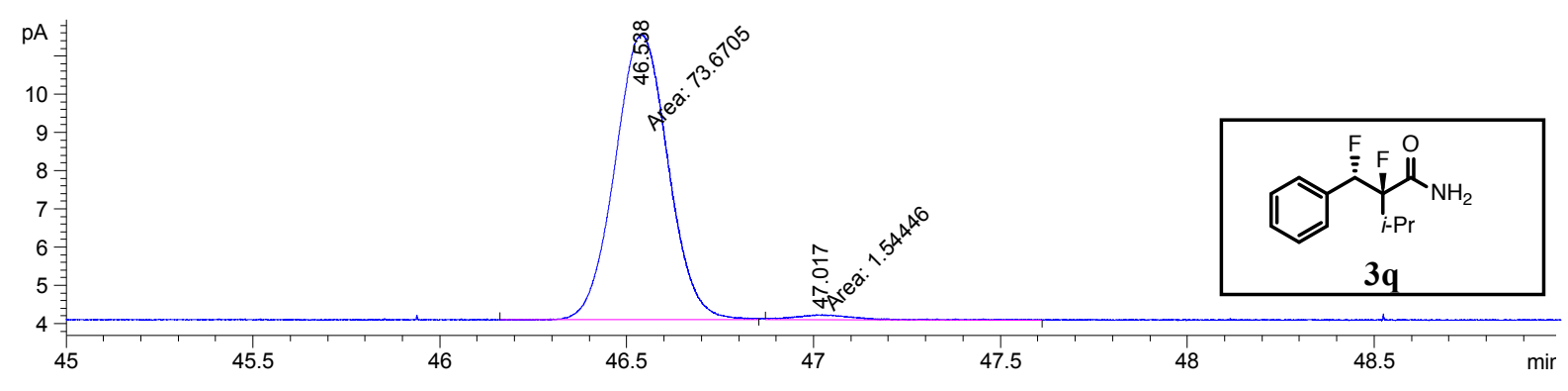

\begin{tabular}{|c|c|c|c|c|c|c|}
\hline $\begin{array}{c}\text { Peak } \\
\#\end{array}$ & $\begin{array}{c}\text { RetTime } \\
\text { [min] }\end{array}$ & Type & $\begin{array}{l}\text { Width } \\
\text { [min] }\end{array}$ & $\begin{array}{r}\text { Area } \\
{\left[\mathrm{pA}^{\star} \mathrm{S}\right]}\end{array}$ & $\begin{array}{l}\text { Height } \\
{[\mathrm{pA}]}\end{array}$ & $\begin{array}{c}\text { Area } \\
\frac{\circ}{0}\end{array}$ \\
\hline & & & & & & \\
\hline 1 & 46.538 & $\mathrm{MF}$ & 543 & 73.67052 & $\begin{array}{l}47315 \\
33 e-1\end{array}$ & $\begin{array}{r}97.94660 \\
2.05340\end{array}$ \\
\hline
\end{tabular}

The absolute stereochemistry of this product and the trace for a racemic sample have been previously reported. ${ }^{6}$ 


\section{References and Notes}

(1) Hu, X.-H.; Zhang, J.; Yang, X.-F.; Xu, Y.-H.; Loh, T.-P. Stereo- and Chemoselective Cross-Coupling between Two Electron-Deficient Acrylates: An Efficient Route to (Z,E)-Muconate Derivatives. J. Am. Chem. Soc. 2015, 137, 3169.

(2) Basavaiah, D.; Krishnamacharyulu, M.; Suguna Hyma, R.; Sarma, P. K. S.; Kumaragurubaran, N. A Facile One-Pot Conversion of Acetates of the Baylis-Hillman Adducts to [E]- $\alpha$-Methylcinnamic Acids. J. Org. Chem. 1999, 64, 1197.

(3) Banik, S. M.; Medley, J. W.; Jacobsen, E. N. Catalytic, asymmetric difluorination of alkenes to generate difluoromethylated stereocenters. Science 2016, 353, 51.

(4) Gensler, W. J.; Berman, E. Decarboxylative Condensation. $\alpha$-Alkylcinnamic Acids from Aromatic Aldehydes and Alkylmalonic Acids. J. Am. Chem. Soc. 1958, 80, 4949.

(5) Hoen, R.; Boogers, J. A. F.; Bernsmann, H.; Minnaard, A. J.; Meetsma, A.; Tiemersma-Wegman, T. D.; de Vries, A. H. M.; de Vries, J. G.; Feringa, B. L. Achiral Ligands Dramatically Enhance Rate and Enantioselectivity in the $\mathrm{Rh} /$ Phosphoramidite-Catalyzed Hydrogenation of $\alpha, \beta$-Disubstituted Unsaturated Acids. Angew. Chem., Int. Ed. 2005, 44, 4209-4212.

(6) Banik, S. M.; Medley, J. W.; Jacobsen, E. N. Catalytic, Diastereoselective 1,2-Difluorination of Alkenes. J. Am. Chem. Soc. 2016, 138, 5000.

(7) Pastushak, N. O.; Dombrovskii, A. V. Zhurnal Obshchei Khimii 1964, 34, 3110.

(8) (a) Taft, R. W., Jr. Linear Free Energy Relationships from Rates of Esterification and Hydrolysis of Aliphatic and Ortho-substituted Benzoate Esters. J. Am. Chem. Soc. 1952, 74, 2729. (b) Taft, R. W., Jr. Steric Eff. Org. Chem. 1956, 556.

(9) Sigman, M. S.; Miller, J. J. Examination of the Role of Taft-Type Steric Parameters in Asymmetric Catalysis. J. Org. Chem. 2009, 74, 7633.

(10) (a) Hirsch, Topics in Stereochemistry, 1967, 3, 199. (b) Jensen, F. R.; Bushweller, C. H. Adv. Alicyclic Chem 1971, 3, 139. (c) Eliel, E. L.; Wilen, S. H.; Stereochemistry of Organic Compounds, Wiley, 1993, p. 696. (d) Eliel, E. L.; Wilen, S. H.; Doyle, M. P. Basic Organic Stereochemistry, Wiley, 2001, p. 443.

(11) Sterimol parameters were obtained using Molecular Modeling Pro Version 6.3.6.

(12) The amide carbonyl IR stretching absorption for $\mathbf{2 a}$ was obtained from: Lewis, F. D.; Elbert, J. E.; Upthagrove, A. L.; Hale, P. D. Structure and Photoisomerization of $(E)$ - and $(Z)$-Cinnamamides and Their Lewis Acid Complexes. J. Org. Chem. 1991, 56, 553. 


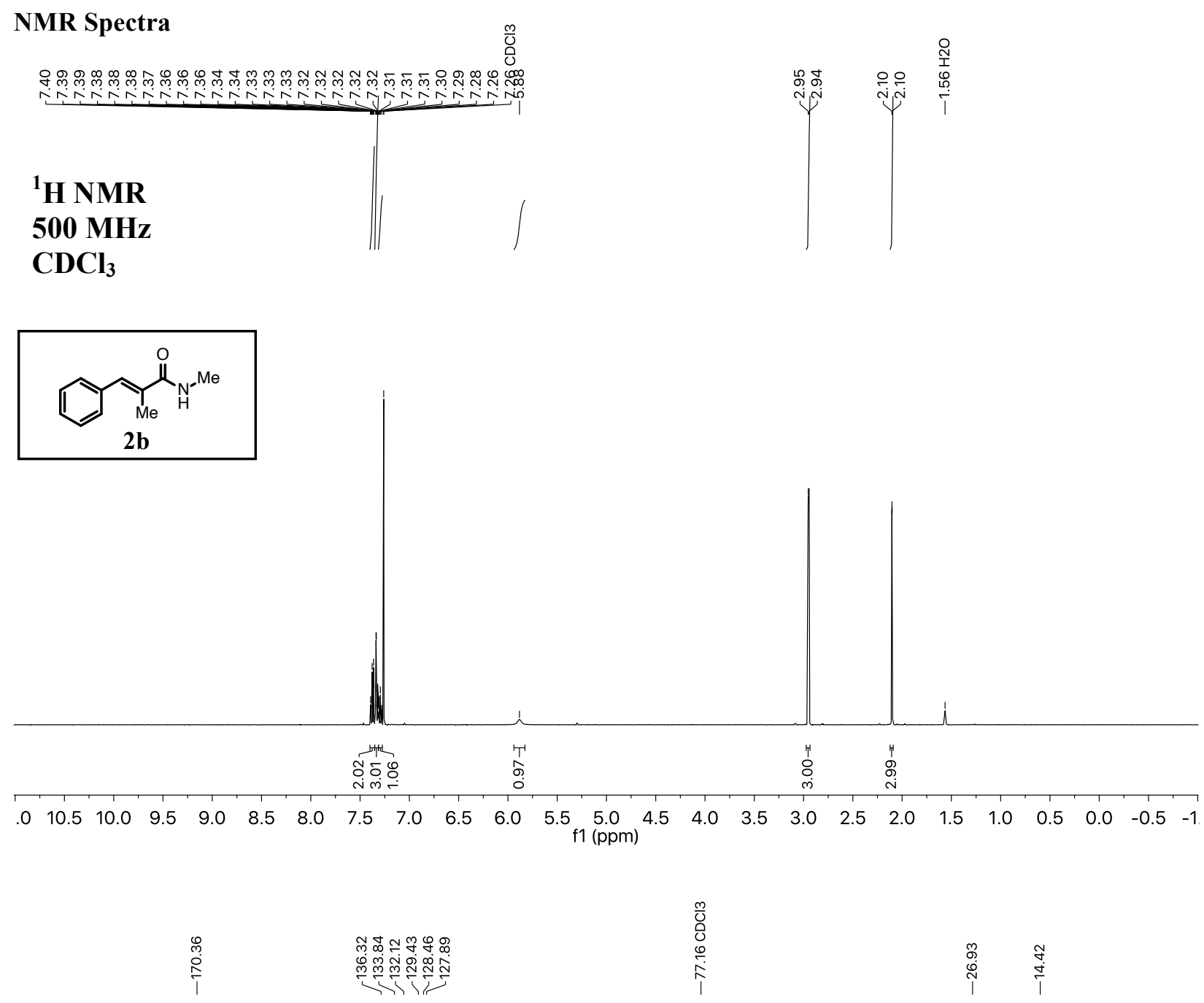

\section{${ }^{13}$ C NMR}

125.7 MHz

$\mathrm{CDCl}_{3}$

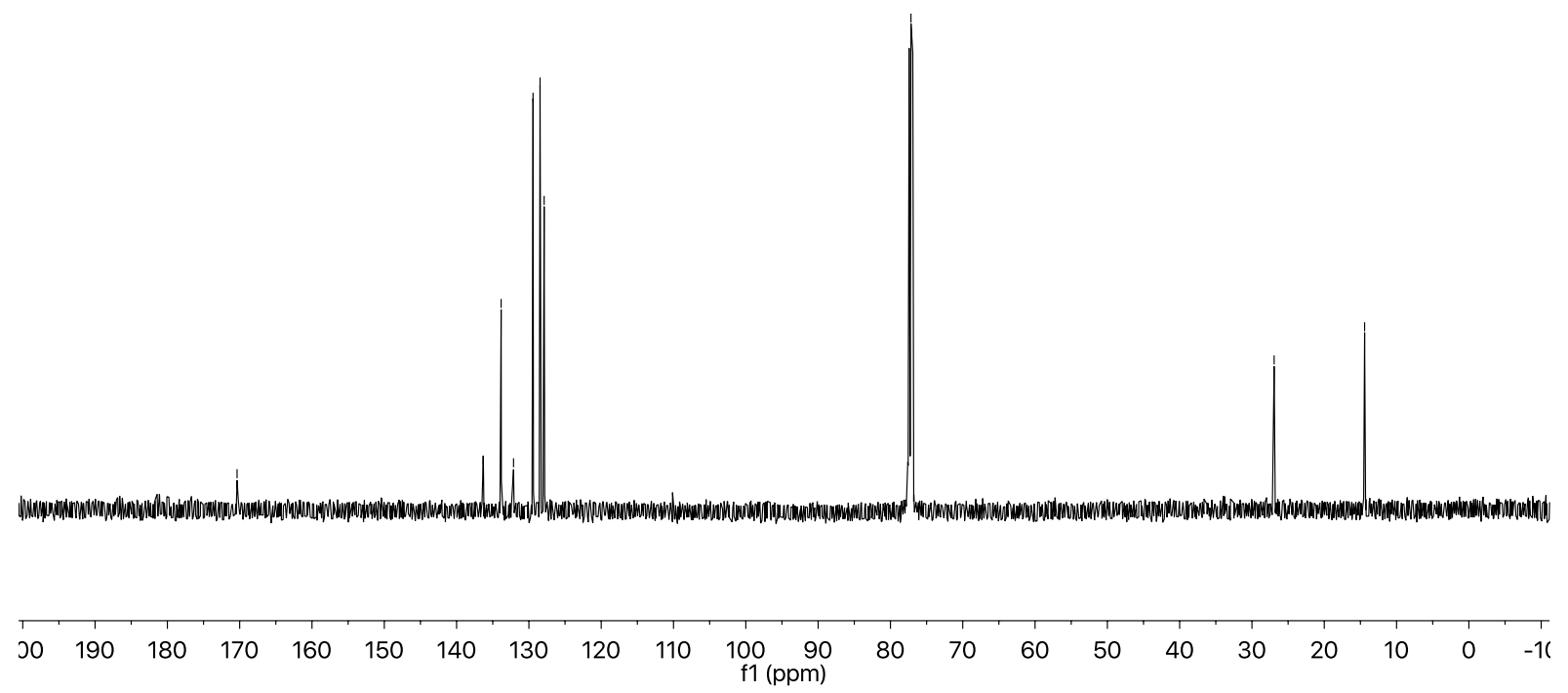



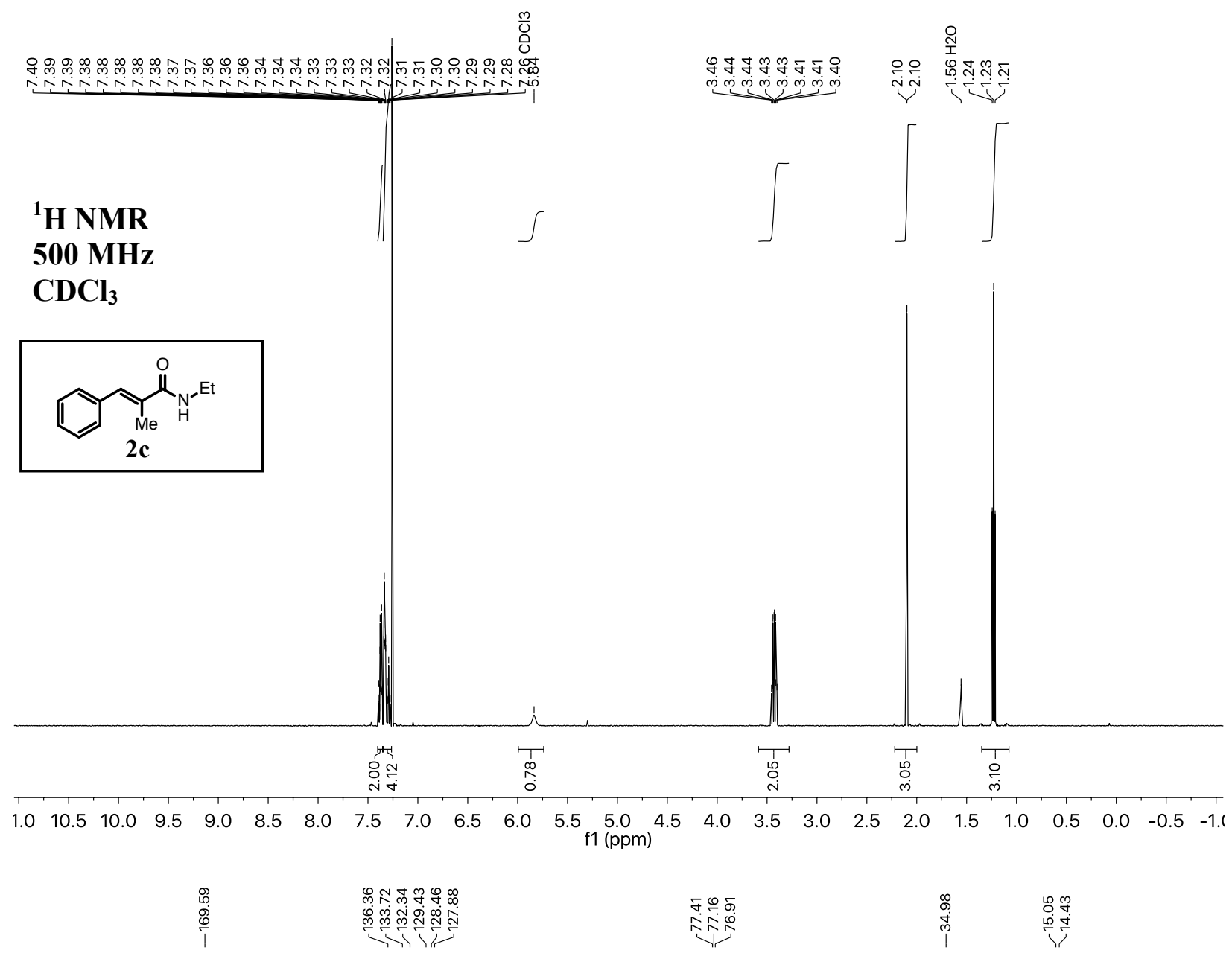

${ }^{13}$ C NMR

125.7 MHz

$\mathrm{CDCl}_{3}$

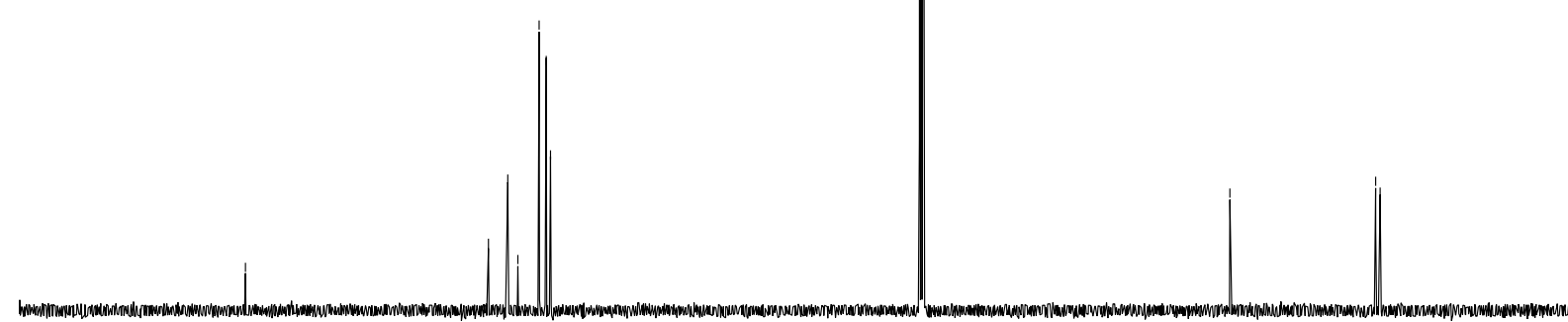

$\begin{array}{llllllllllllllllllllll}\text { J0 } & 190 & 180 & 170 & 160 & 150 & 140 & 130 & 120 & 110 & \begin{array}{r}100 \\ f 1(\mathrm{ppm})\end{array} & 80 & 70 & 60 & 50 & 40 & 30 & 20 & 10 & 0 & -1 \mathrm{C}\end{array}$ 

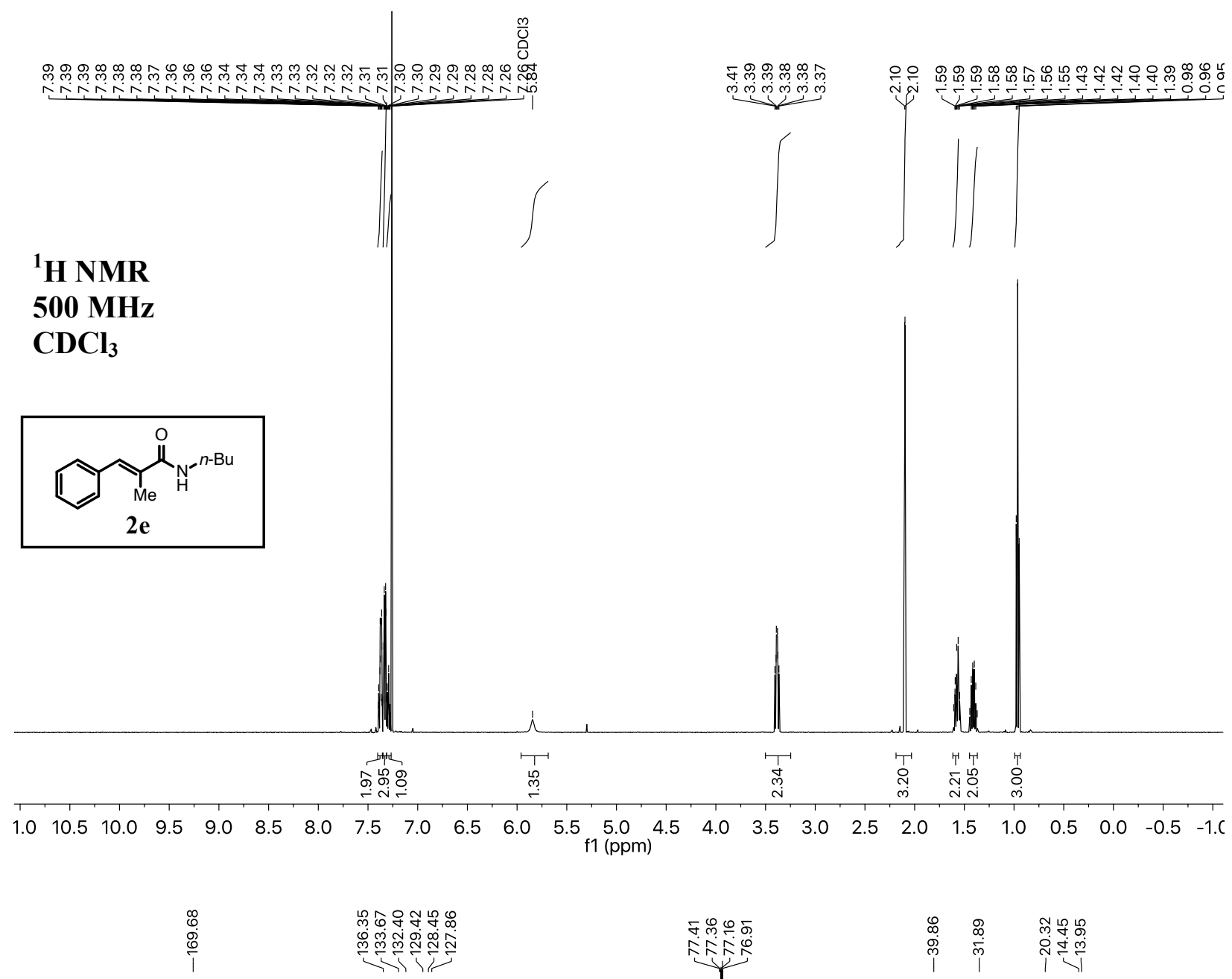

${ }^{13} \mathrm{C}$ NMR

125. $7 \mathrm{MHz}$

$\mathrm{CDCl}_{3}$

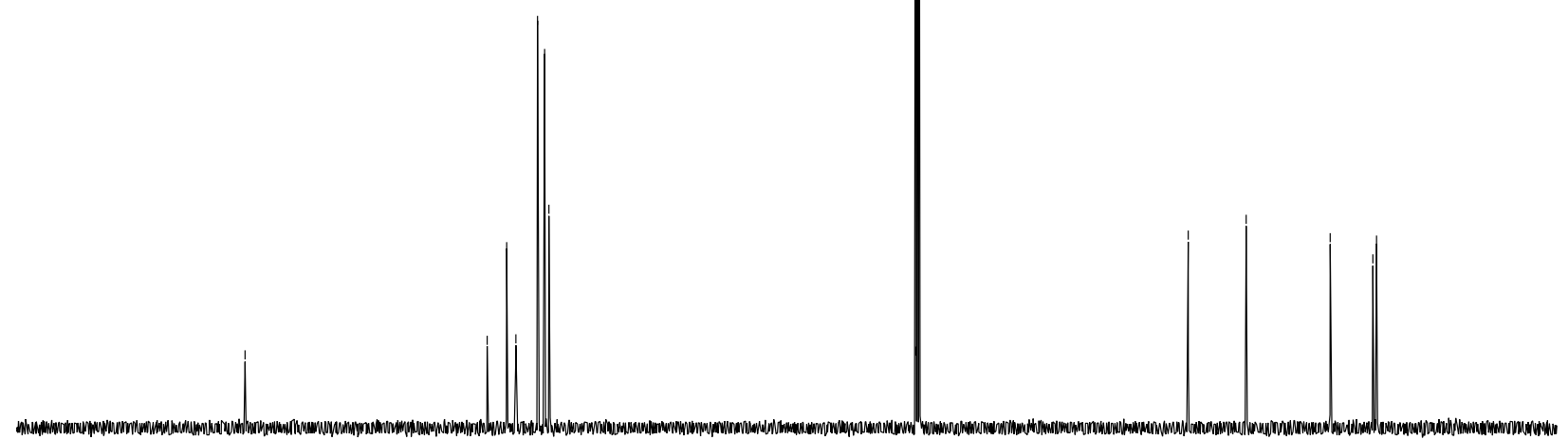

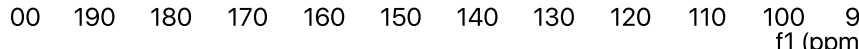



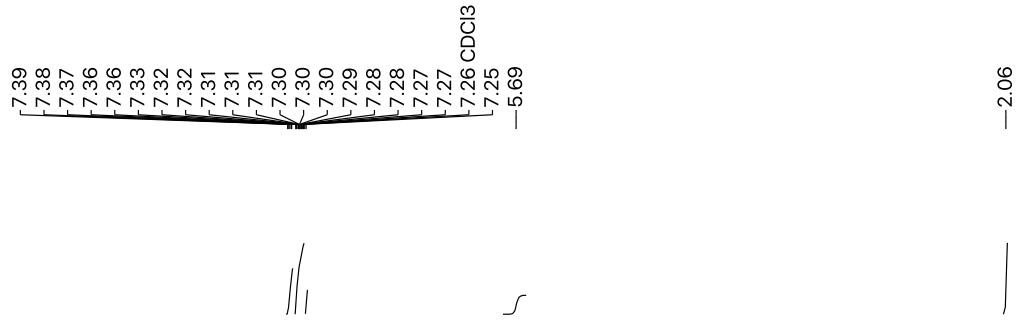

${ }^{1}$ H NMR

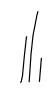

$500 \mathrm{MHz}$

$\mathrm{CDCl}_{3}$

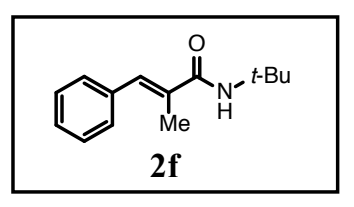

\section{${ }^{13} \mathrm{C}$ NMR}

125.7 MHz

\section{$\mathrm{CDCl}_{3}$}

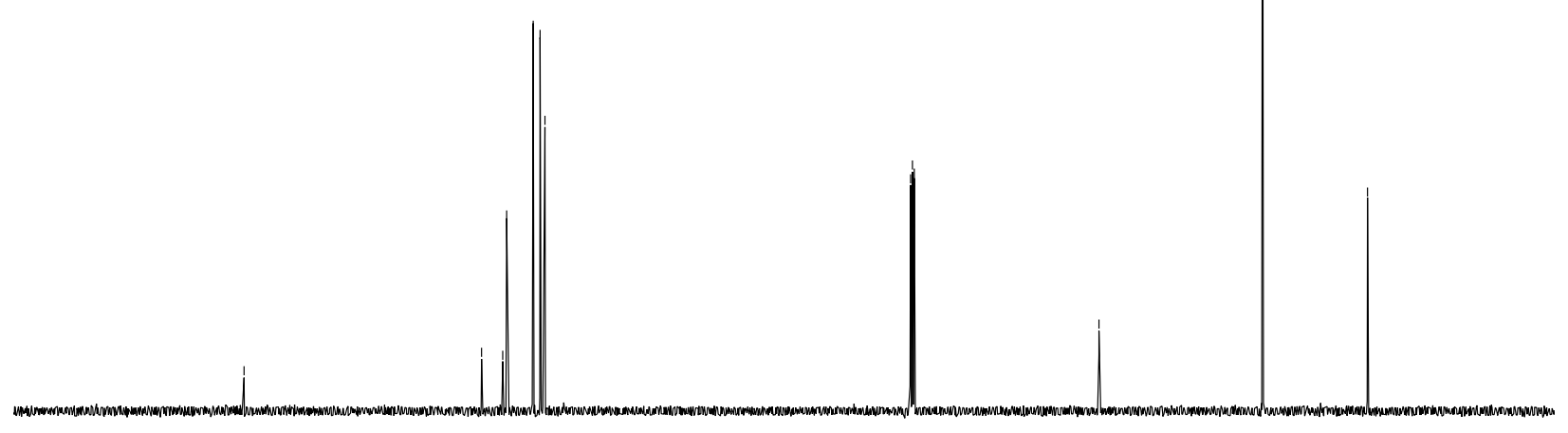

\begin{tabular}{lllllllllllllllllllll}
\hline 00 & 190 & 180 & 170 & 160 & 150 & 140 & 130 & 120 & 110 & $\underset{f 1}{100} \underset{(\mathrm{ppm})}{90}$ & 80 & 70 & 60 & 50 & 40 & 30 & 20 & 10 & 0 & -16
\end{tabular} 


\section{${ }^{1}$ H NMR}

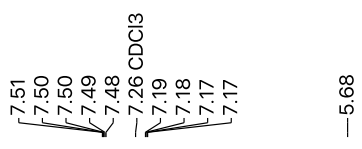

$\infty$
0
0
$\dot{0}$

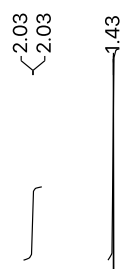

$500 \mathrm{MHz}$

$\mathrm{CDCl}_{3}$
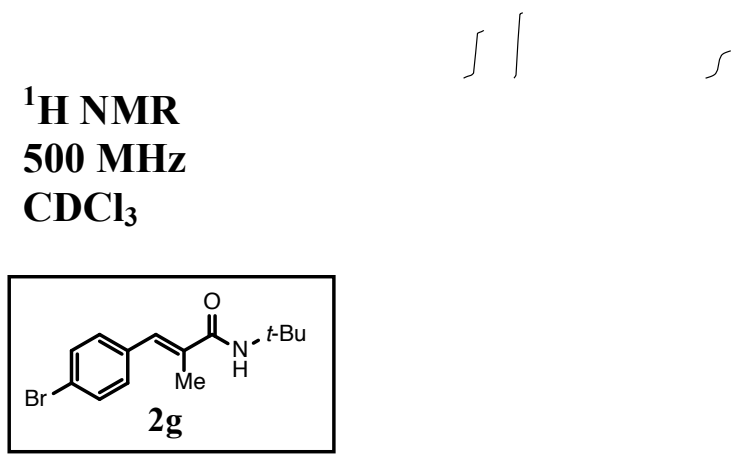

s 


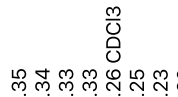

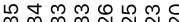

选代然

فํ.

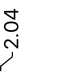

${ }^{1}$ H NMR

$500 \mathrm{MHz}$

$\mathrm{CDCl}_{3}$
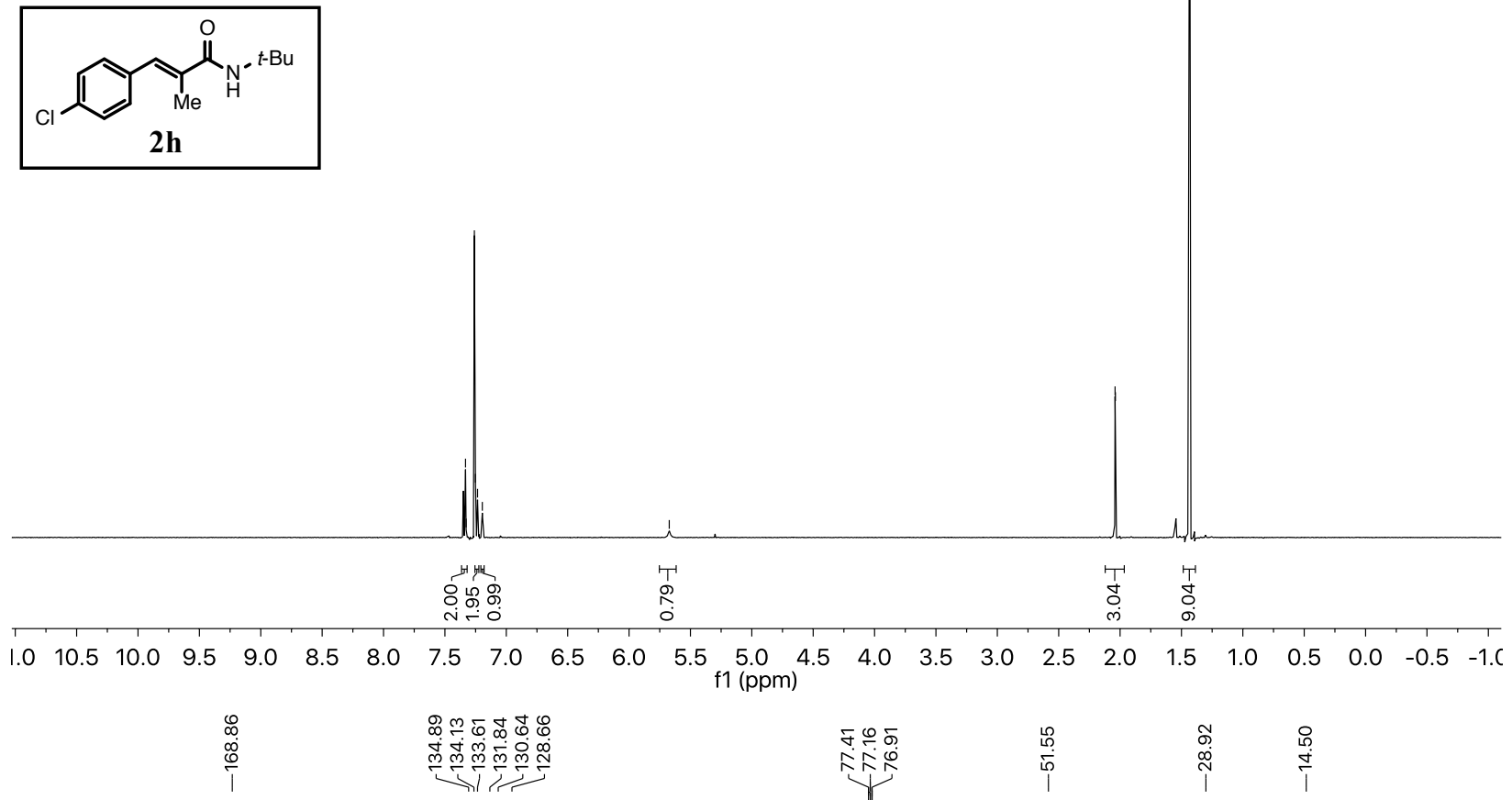

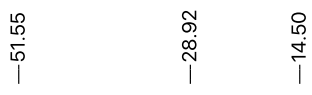

\section{${ }^{13} \mathrm{C}$ NMR}

125.7 MHz

$\mathrm{CDCl}_{3}$
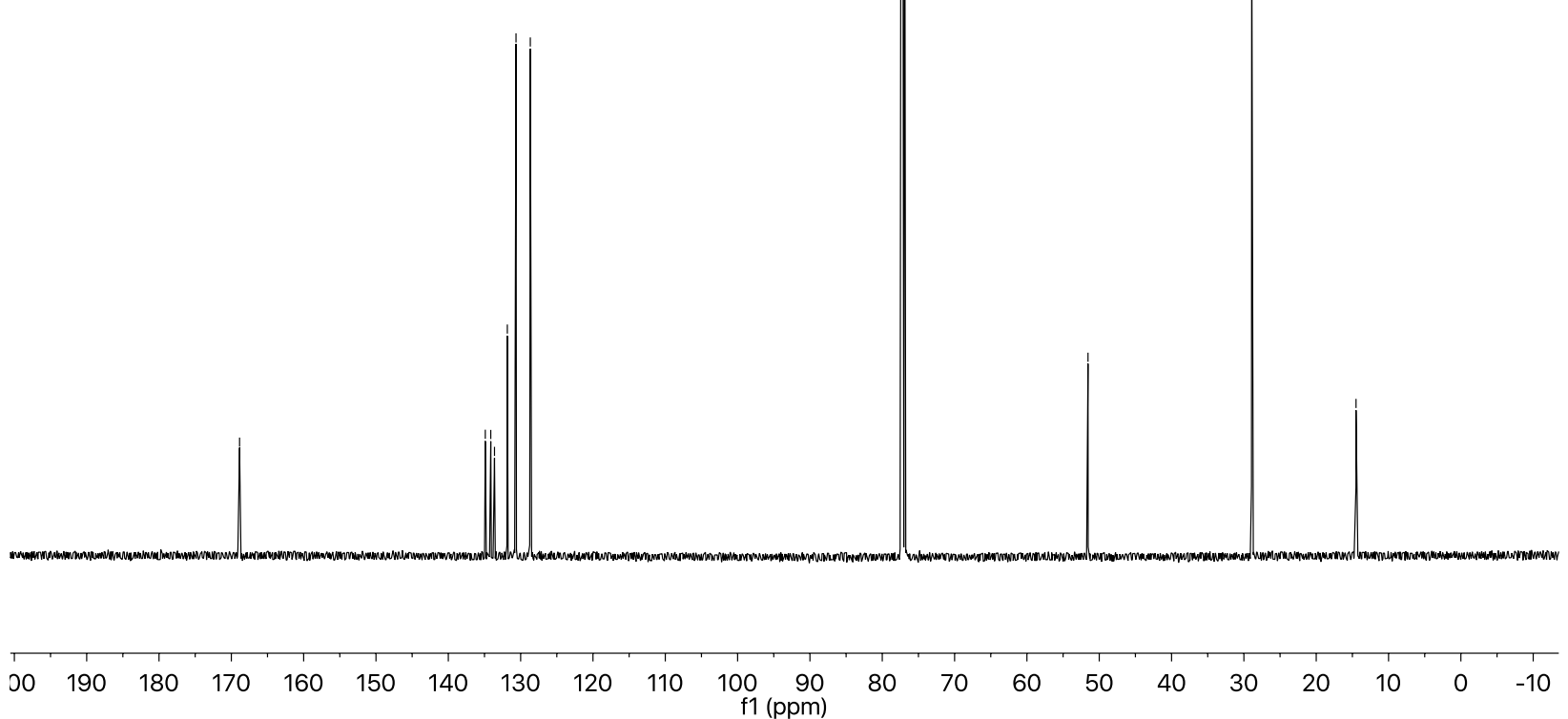
${ }^{1} \mathrm{H}$ NMR

$500 \mathrm{MHz}$

$1 / 1$

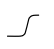

$\mathrm{CDCl}_{3}$
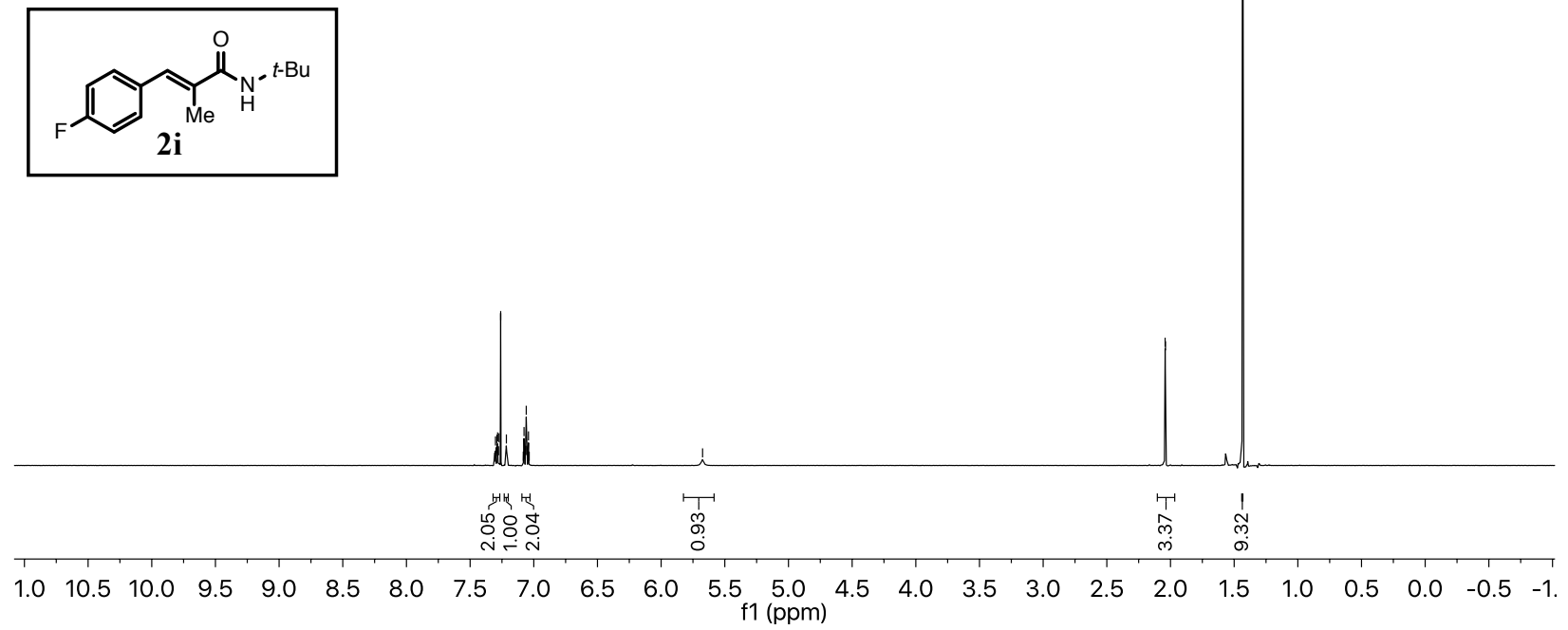

${ }^{13}$ C NMR

125.7 MHz

$\mathrm{CDCl}_{3}$

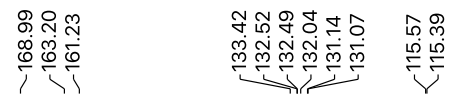

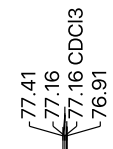

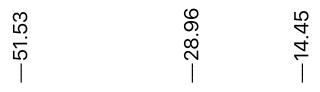

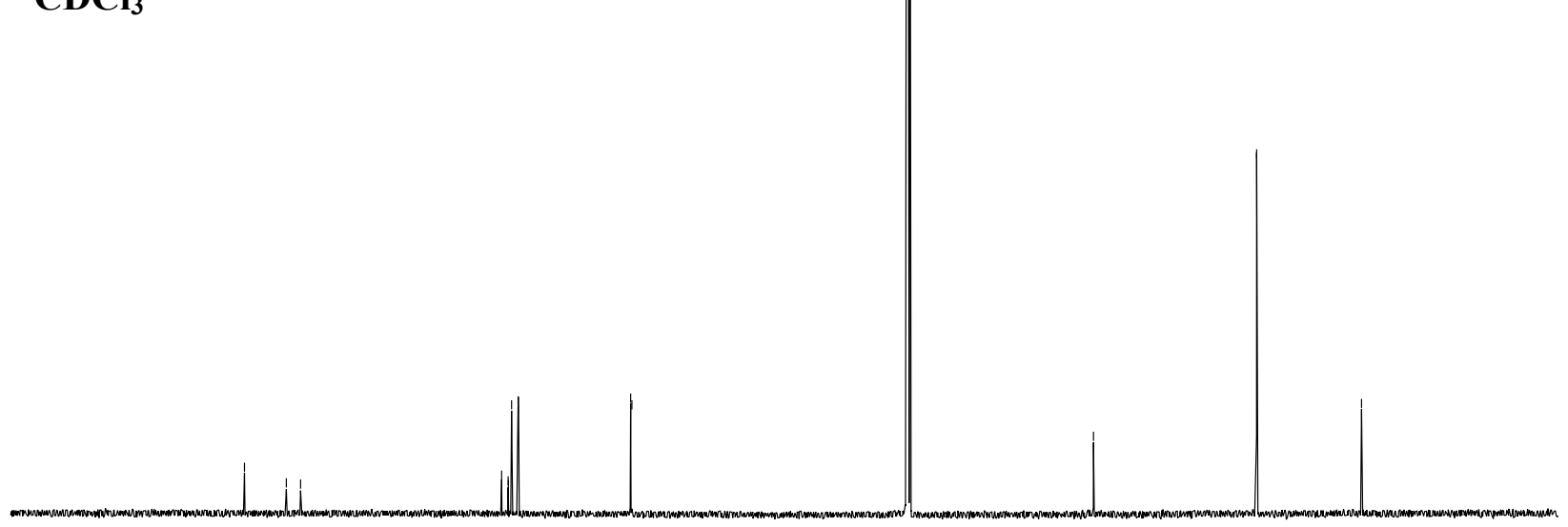

$\begin{array}{llllllllll}190 & 180 & 170 & 160 & 150 & 140 & 130 & 120 & 110 & \begin{array}{r}100 \\ \mathrm{f} 1(\mathrm{ppm})\end{array}\end{array}$

$80 \quad 70 \quad 60$

50

40

$30 \quad 20$

$10 \quad 0 \quad-10$ 


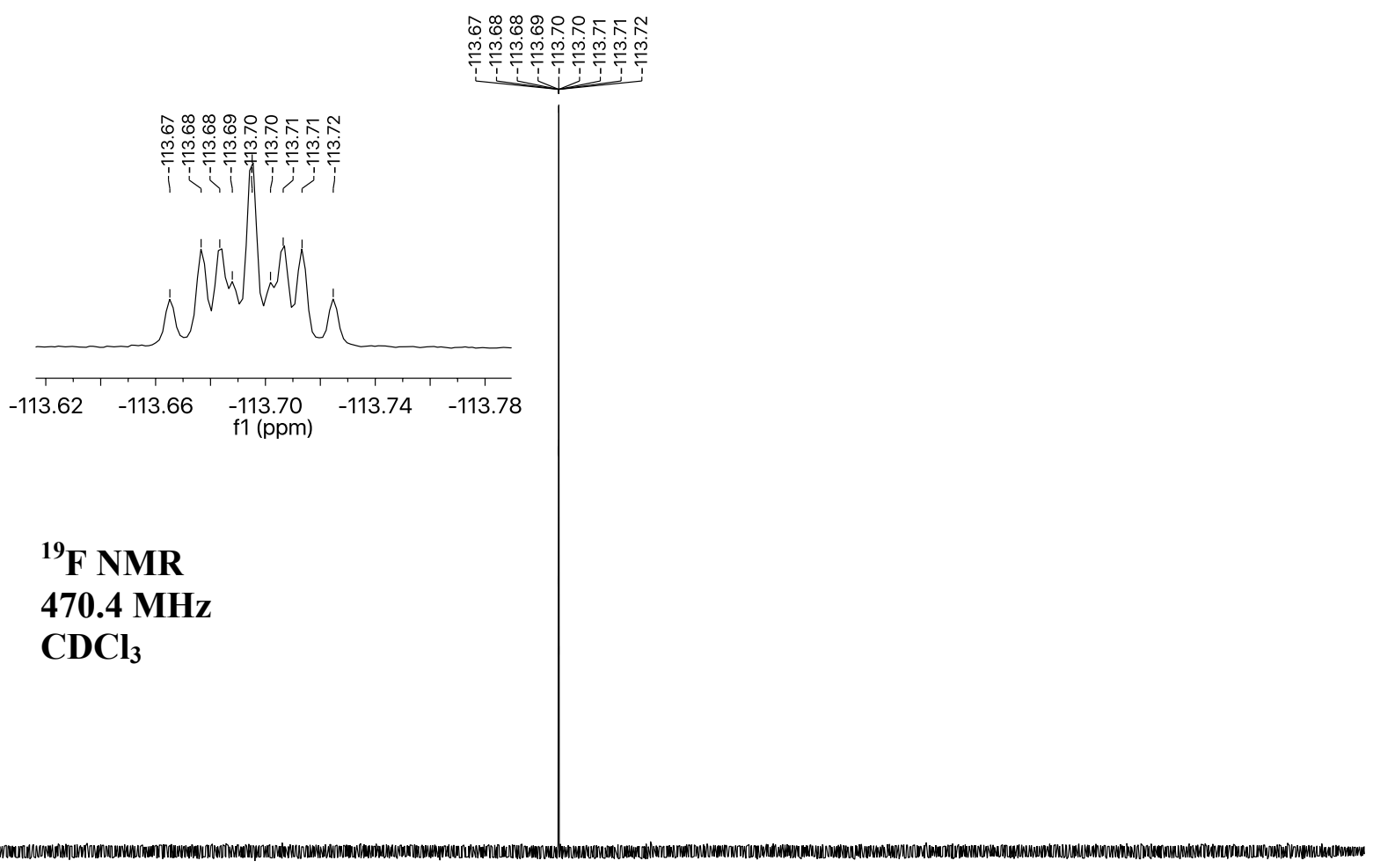

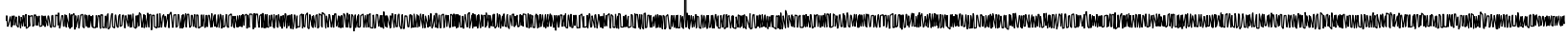

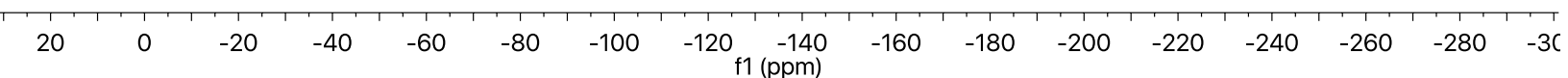




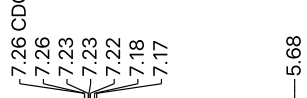

ஸึ

\section{.}

$500 \mathrm{MHz}$

$\mathrm{CDCl}_{3}$
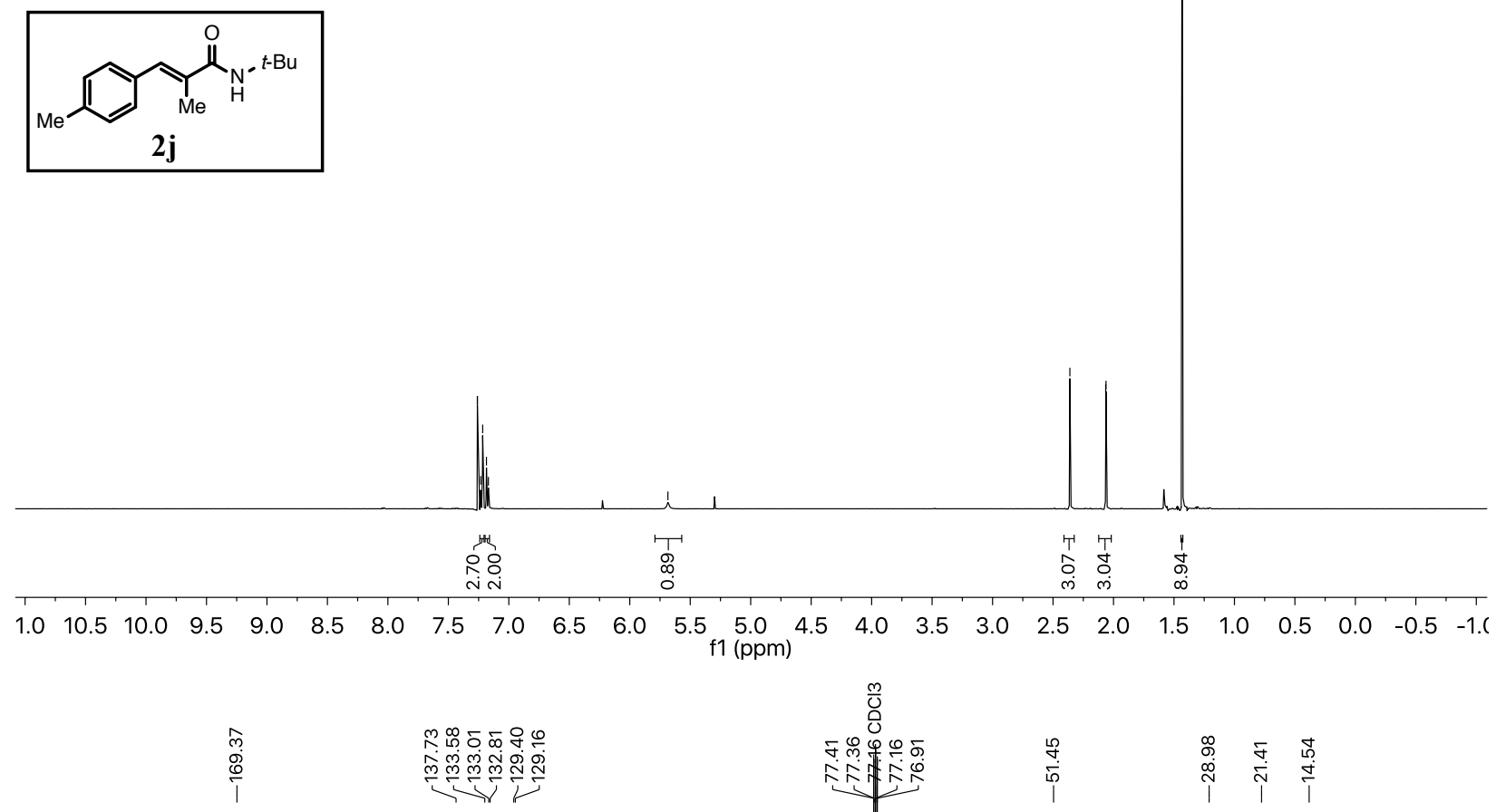

\section{${ }^{13} \mathrm{C}$ NMR}

125.7 MHz

\section{$\mathrm{CDCl}_{3}$}
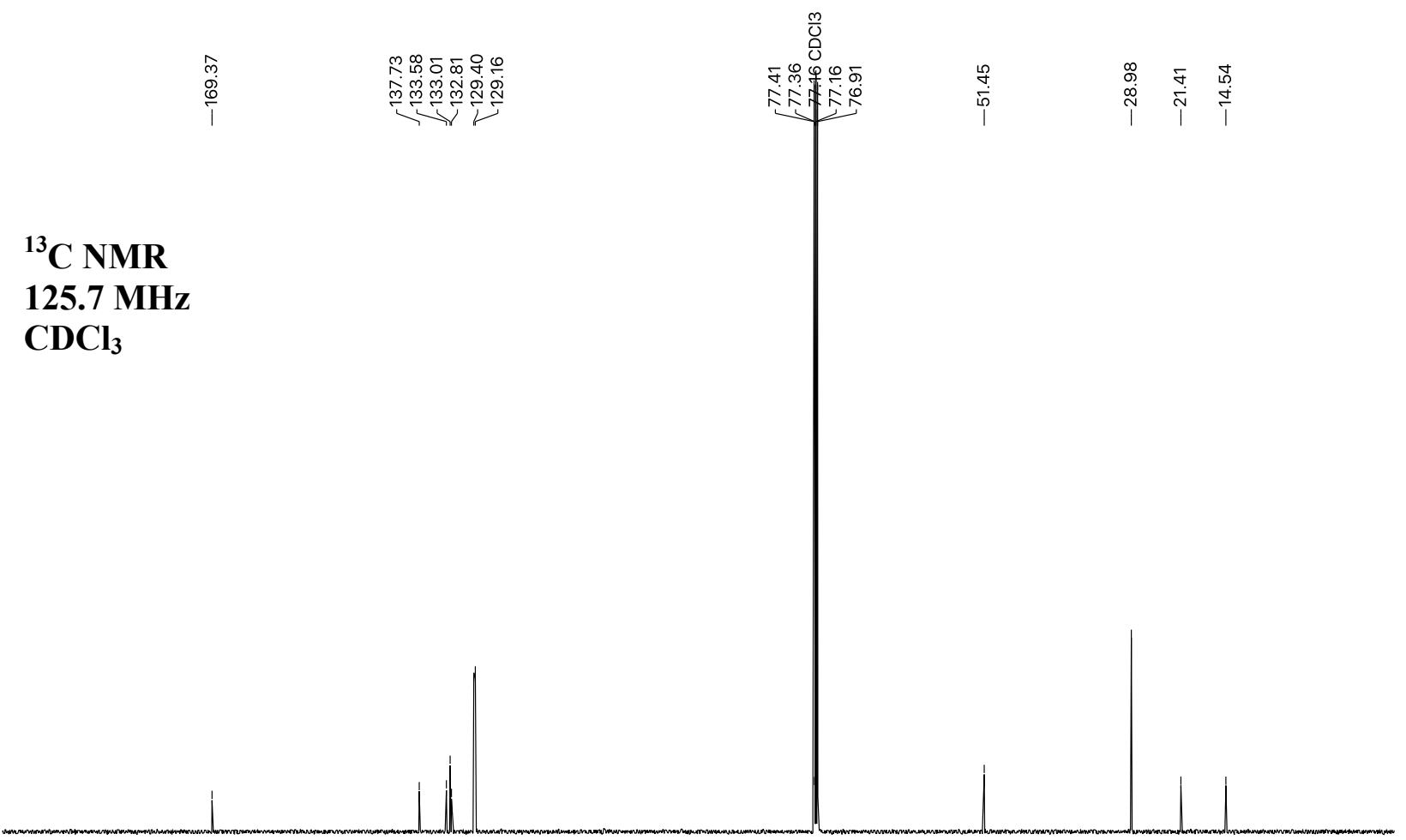

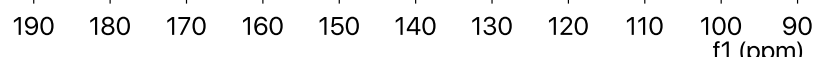

$80 \quad 70 \quad 60$

$50 \quad 40$

$\begin{array}{lllll}30 & 20 & 10 & 0 & -10\end{array}$ 

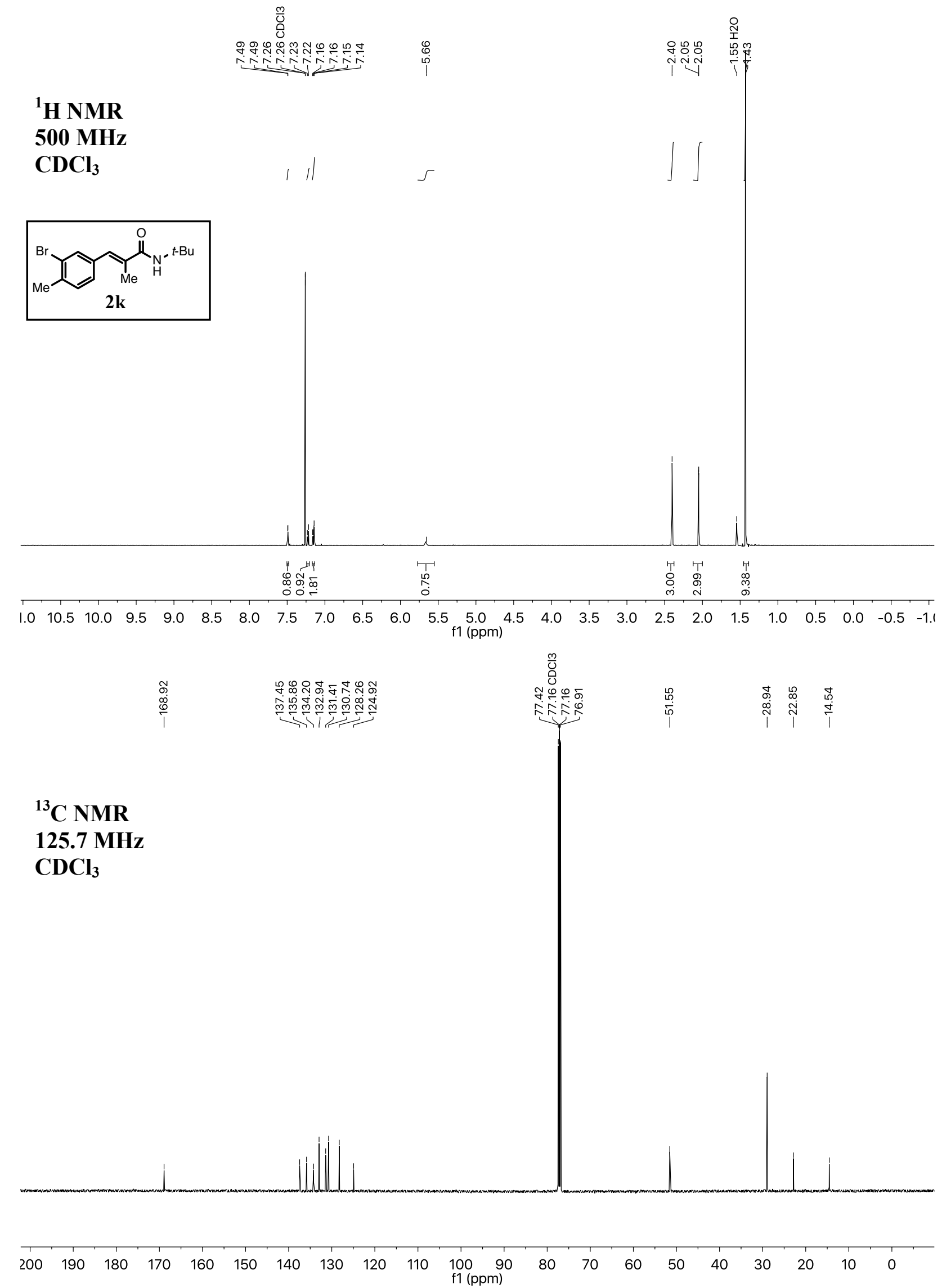

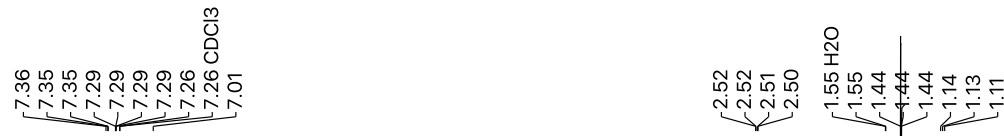

${ }^{1}$ H NMR

$500 \mathrm{MHz}$

$\mathrm{CDCl}_{3}$
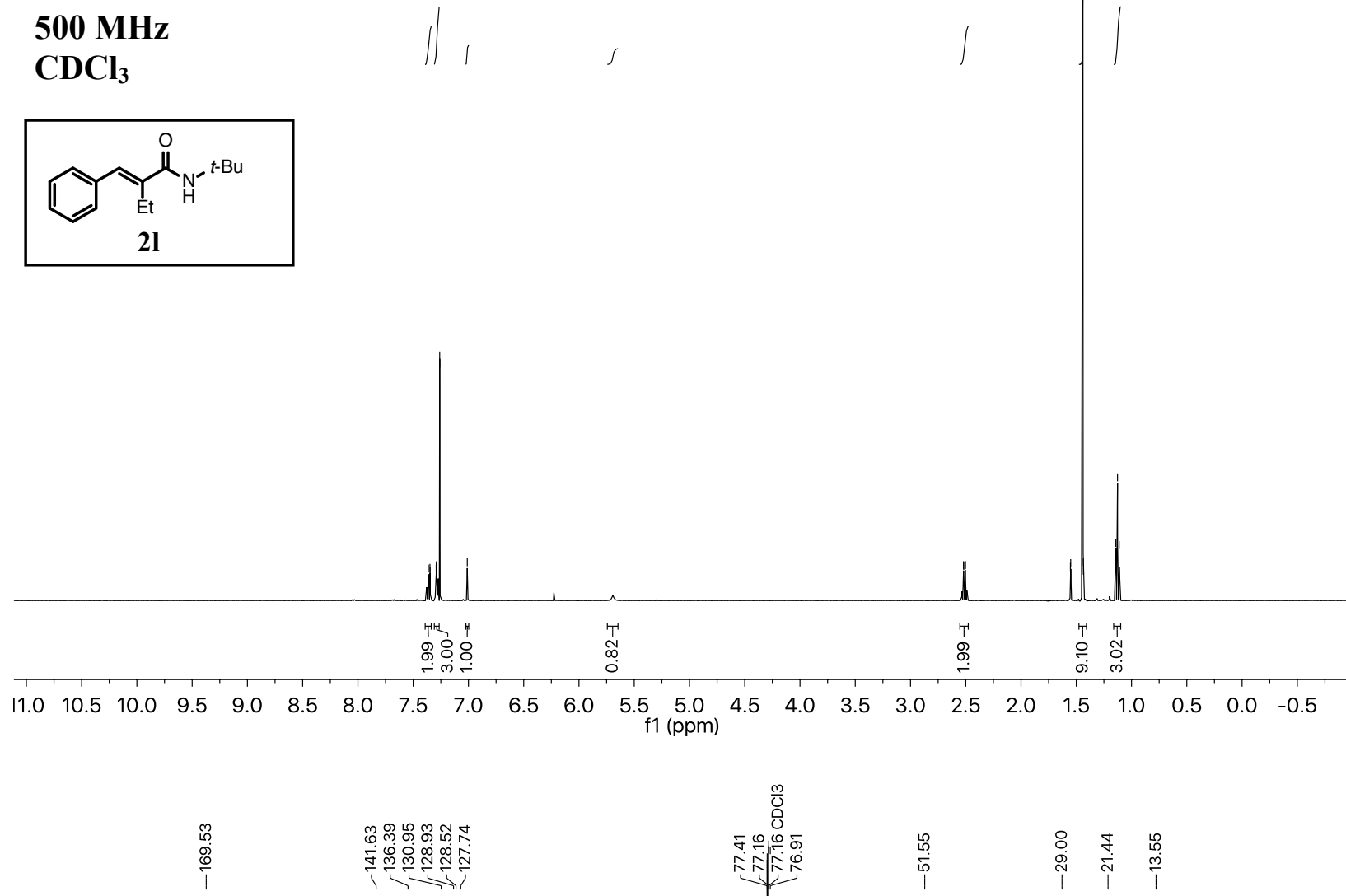

${ }^{13}$ C NMR

125.7 MHz

$\mathrm{CDCl}_{3}$

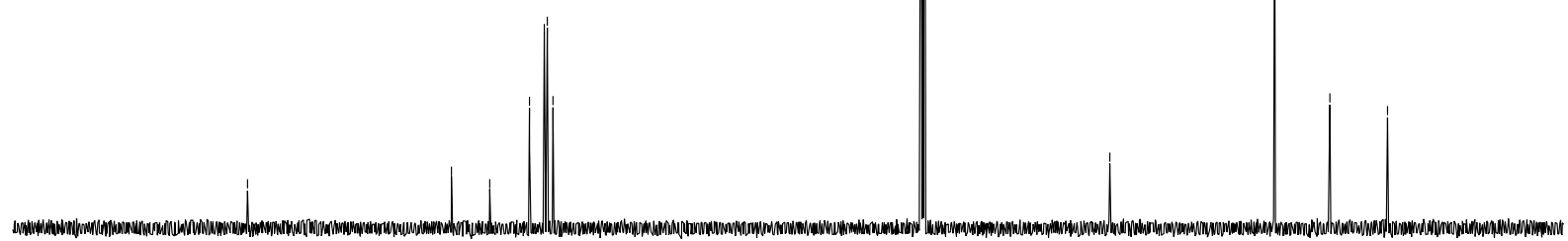

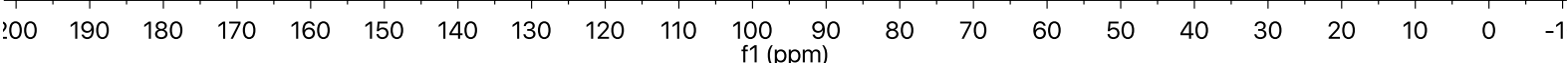



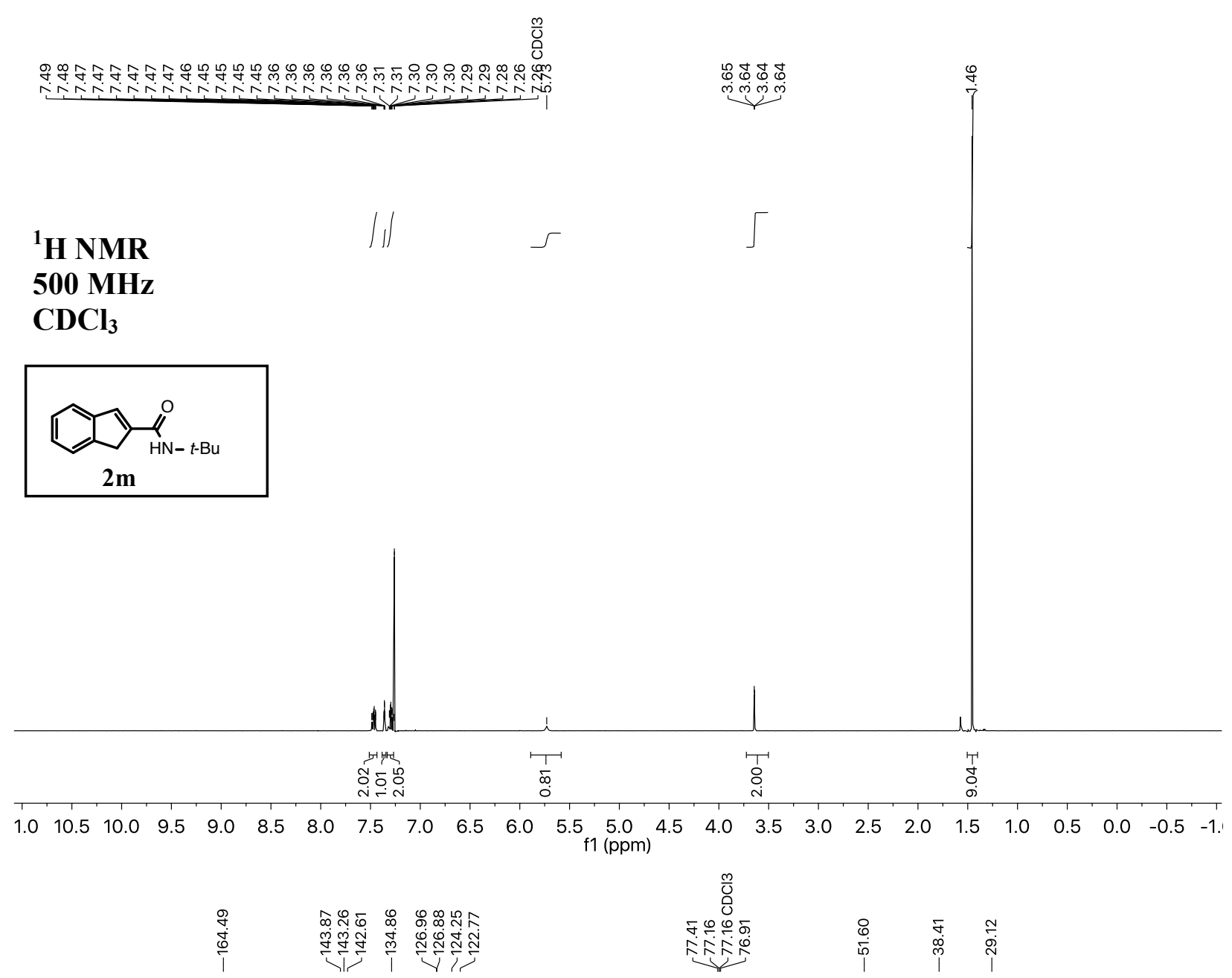

\section{${ }^{13} \mathrm{C}$ NMR}

125.7 MHz

$\mathrm{CDCl}_{3}$

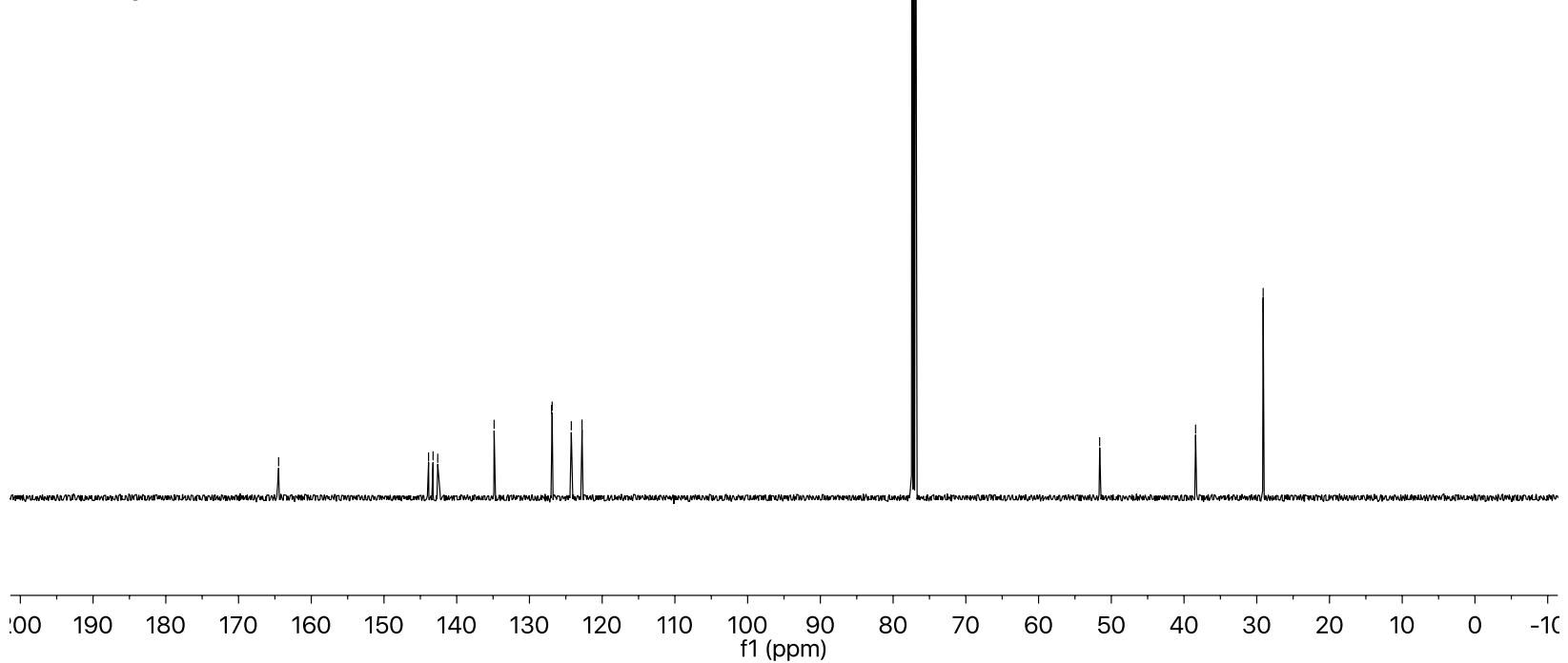



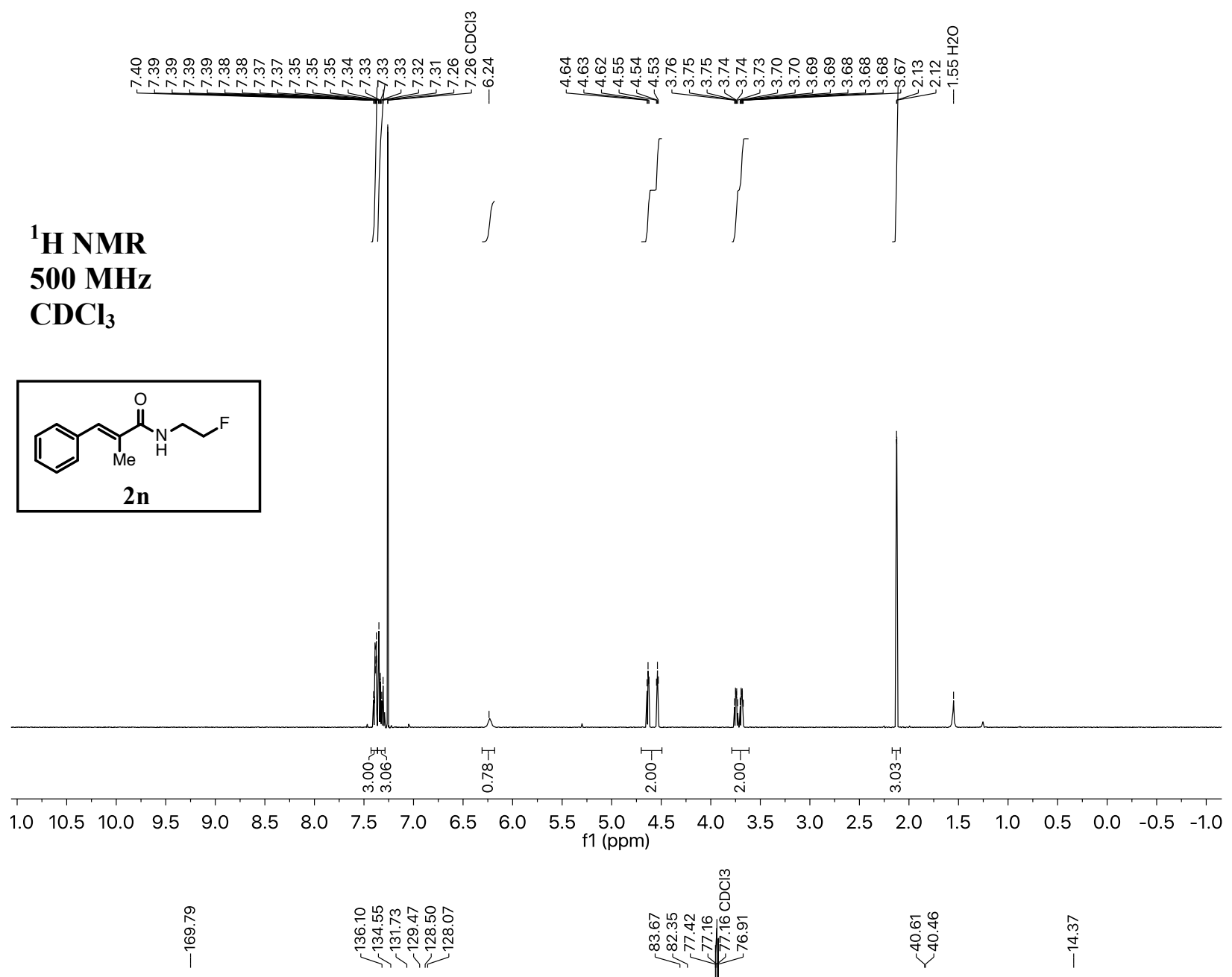

\section{${ }^{13} \mathrm{C}$ NMR \\ 125.7 MHz \\ $\mathrm{CDCl}_{3}$}

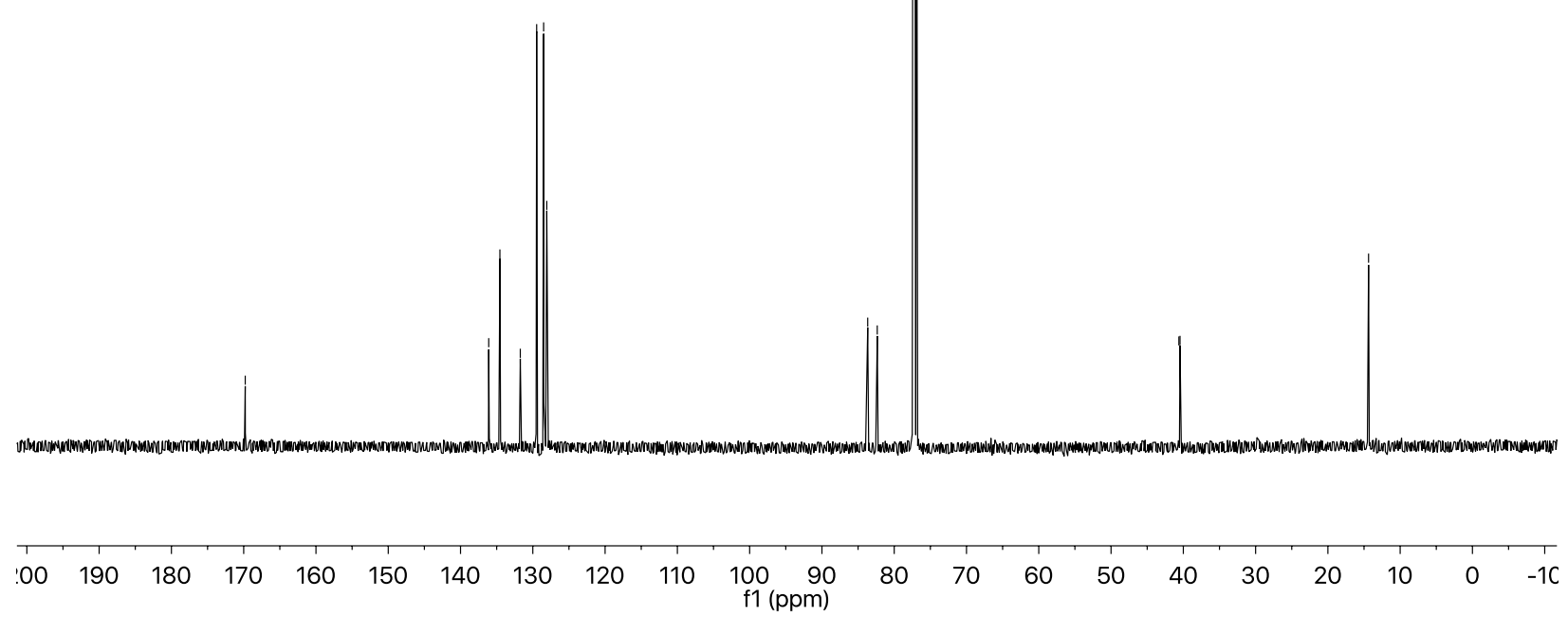




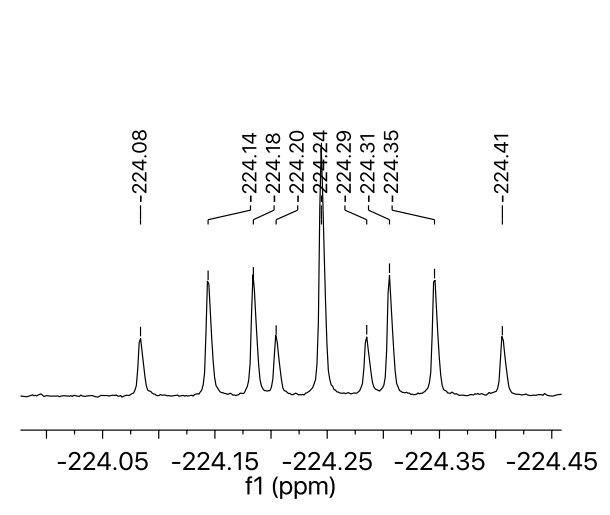

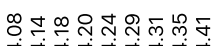

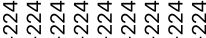

${ }^{19}$ F NMR

470.4 MHz

$\mathrm{CDCl}_{3}$

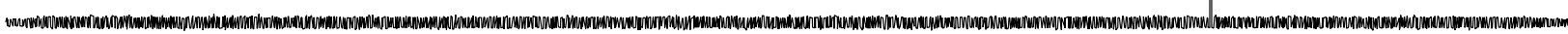

\begin{tabular}{lllllllllllllllllllllllllll}
\hline 20 & 0 & -20 & -40 & -60 & -80 & -100 & -120 & -140 & -160 & -180 & -200 & -220 & -240 & -260 & -280 & -30
\end{tabular}



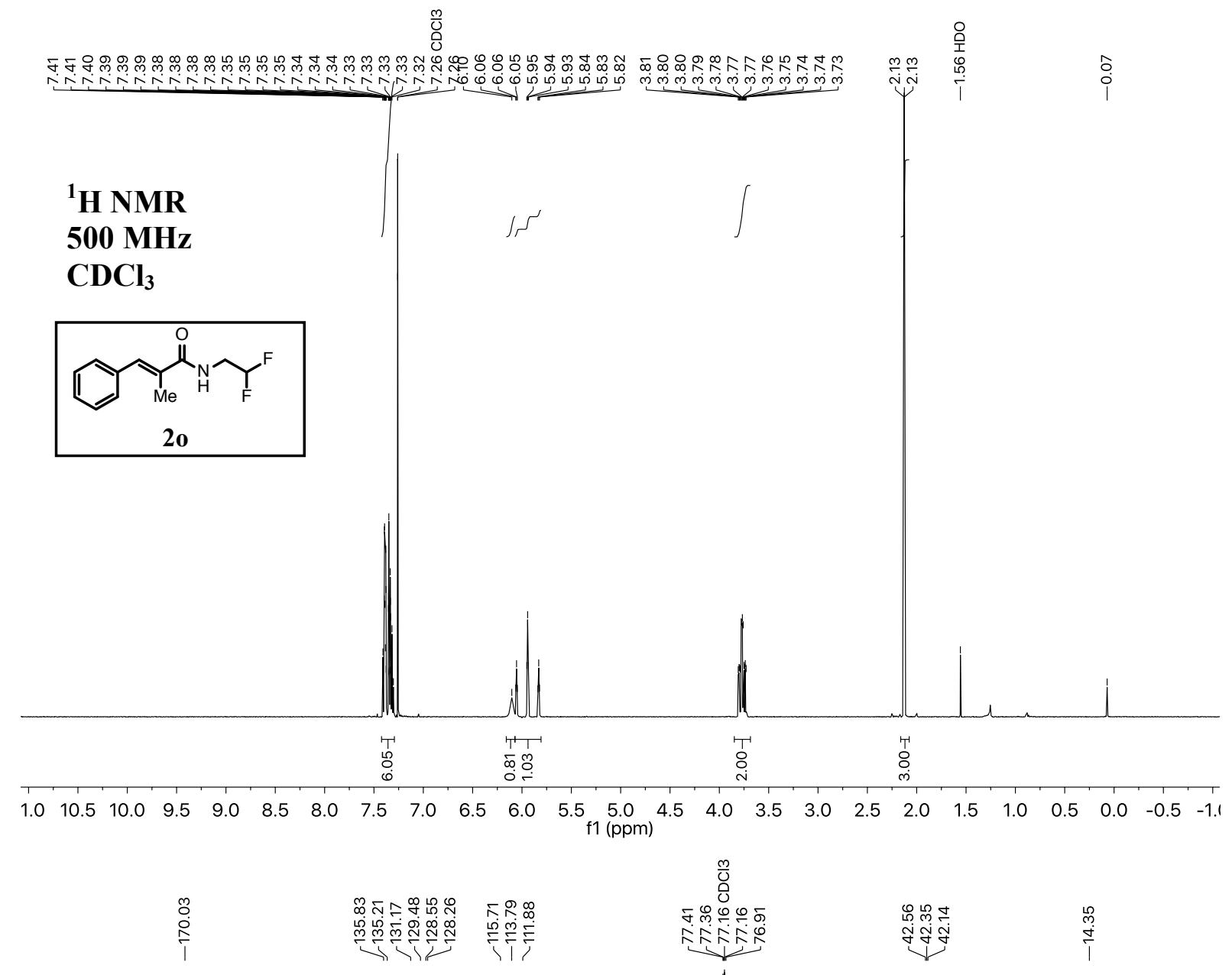

\section{${ }^{13} \mathrm{C}$ NMR \\ 125.7 MHz \\ $\mathrm{CDCl}_{3}$}

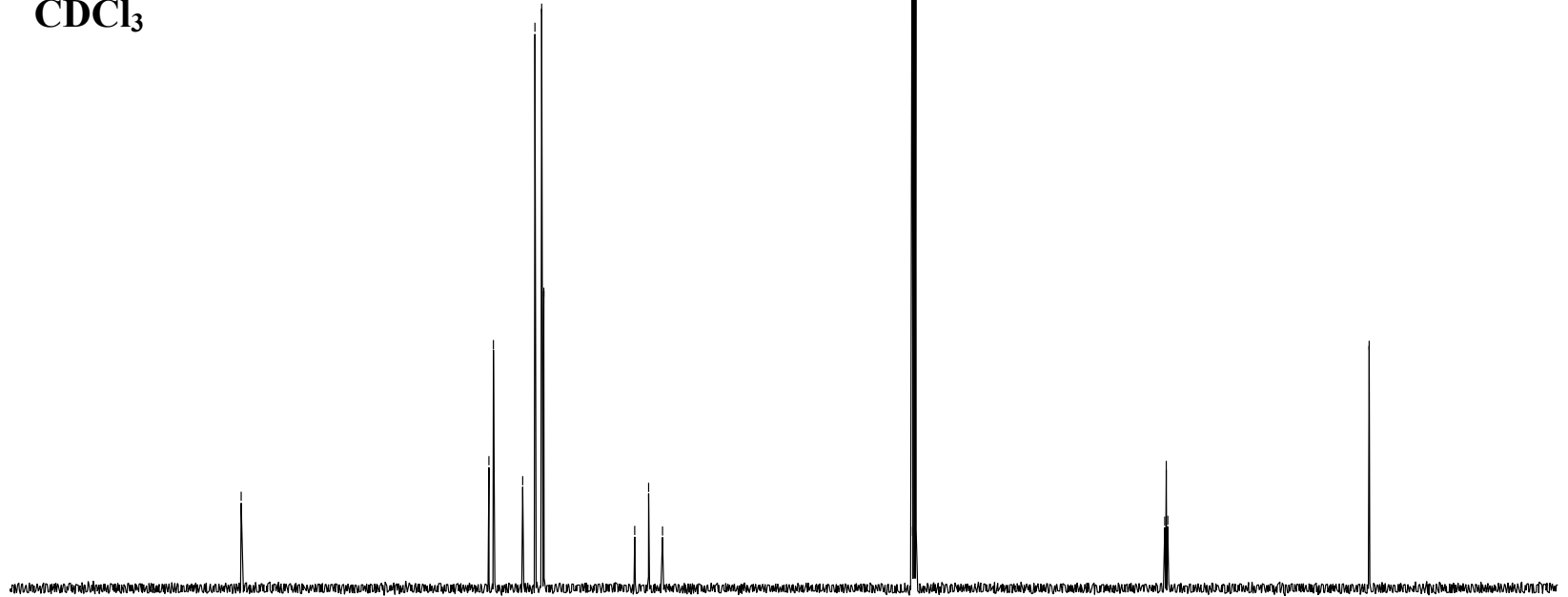

$\begin{array}{lllllllllllllllllllll}200 & 190 & 180 & 170 & 160 & 150 & 140 & 130 & 120 & 110 & \begin{array}{c}100 \\ \mathrm{f} 1(\mathrm{ppm})\end{array} & 80 & 70 & 60 & 50 & 40 & 30 & 20 & 10 & 0 & -1 \mathrm{C}\end{array}$ 

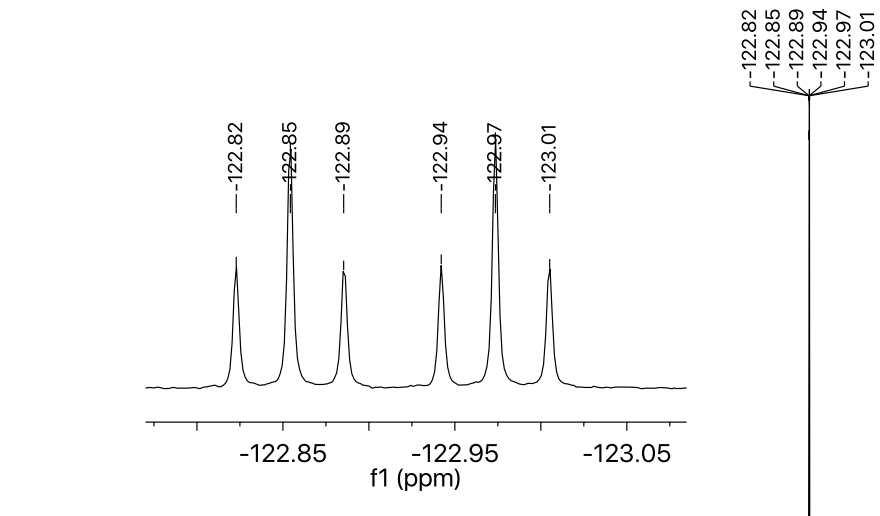

\section{${ }^{19}$ F NMR \\ 470.4 MHz \\ $\mathrm{CDCl}_{3}$}

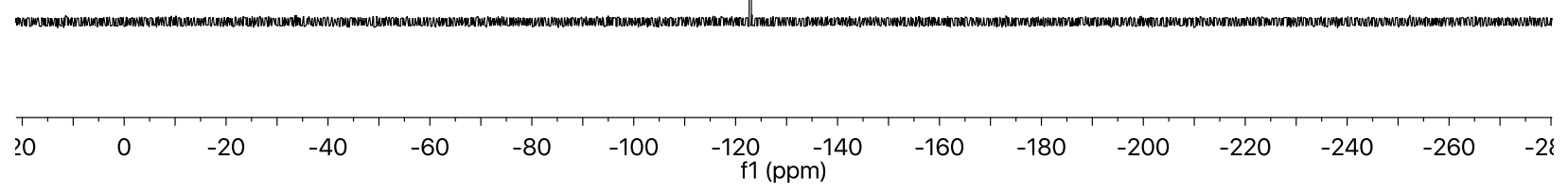



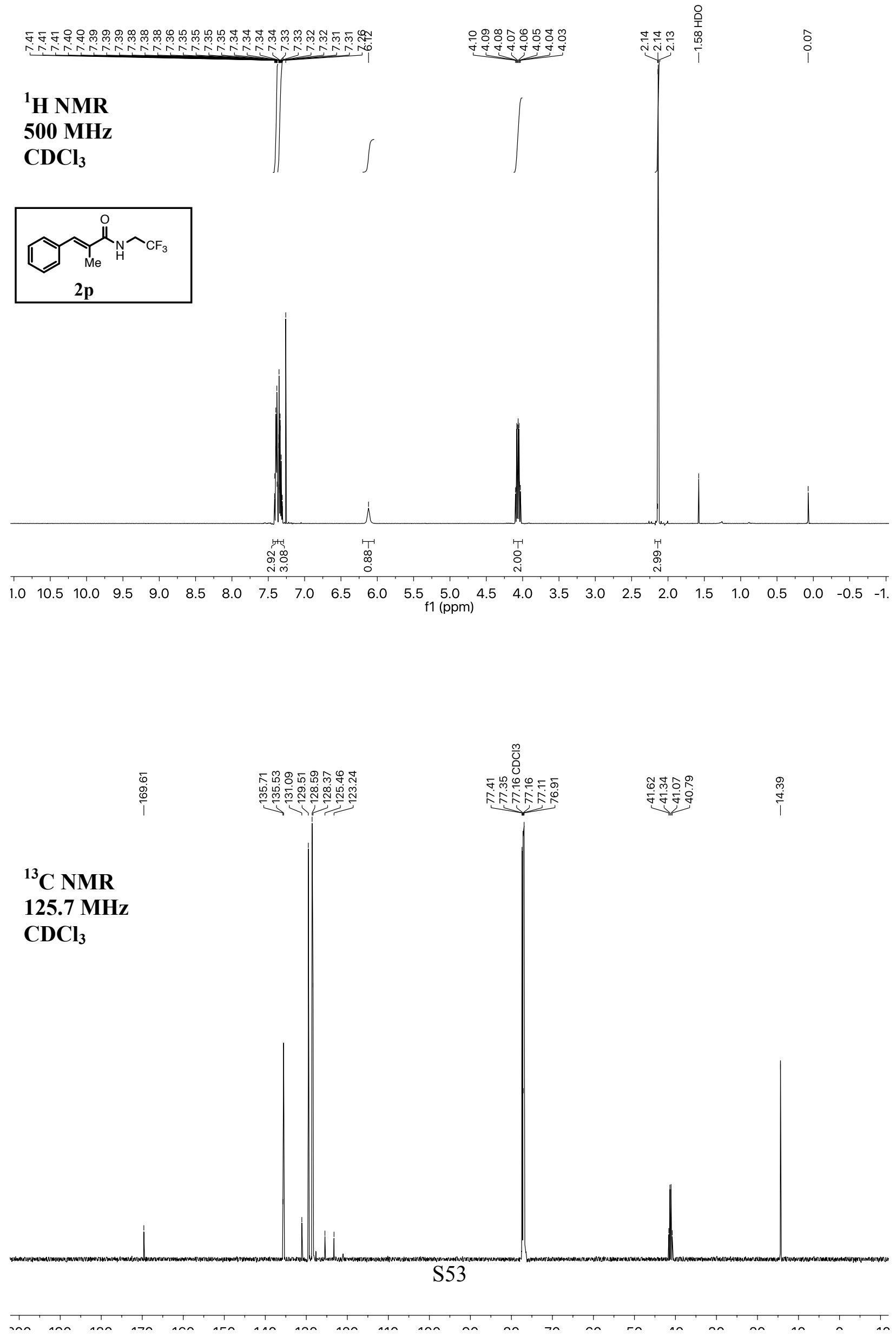


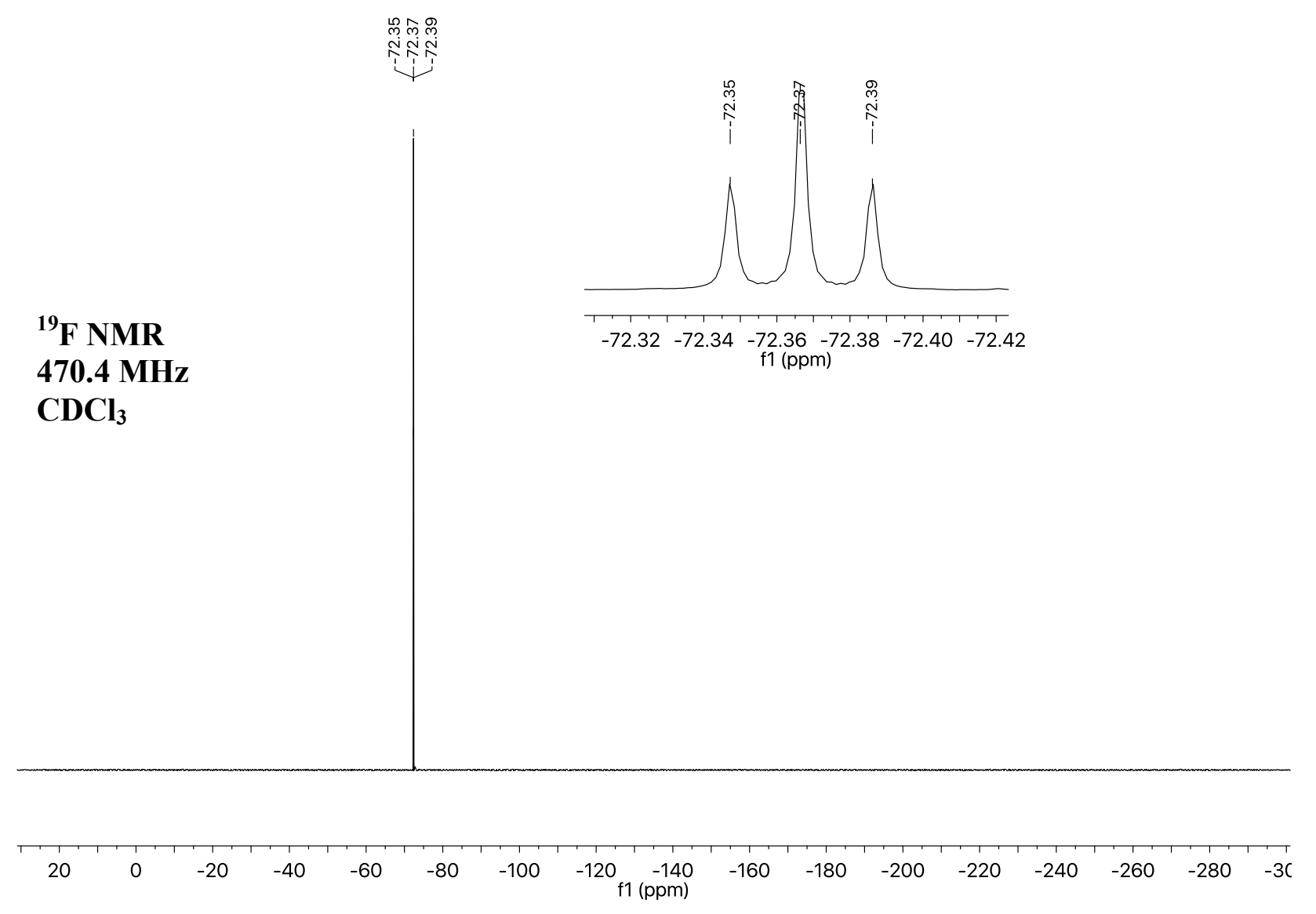



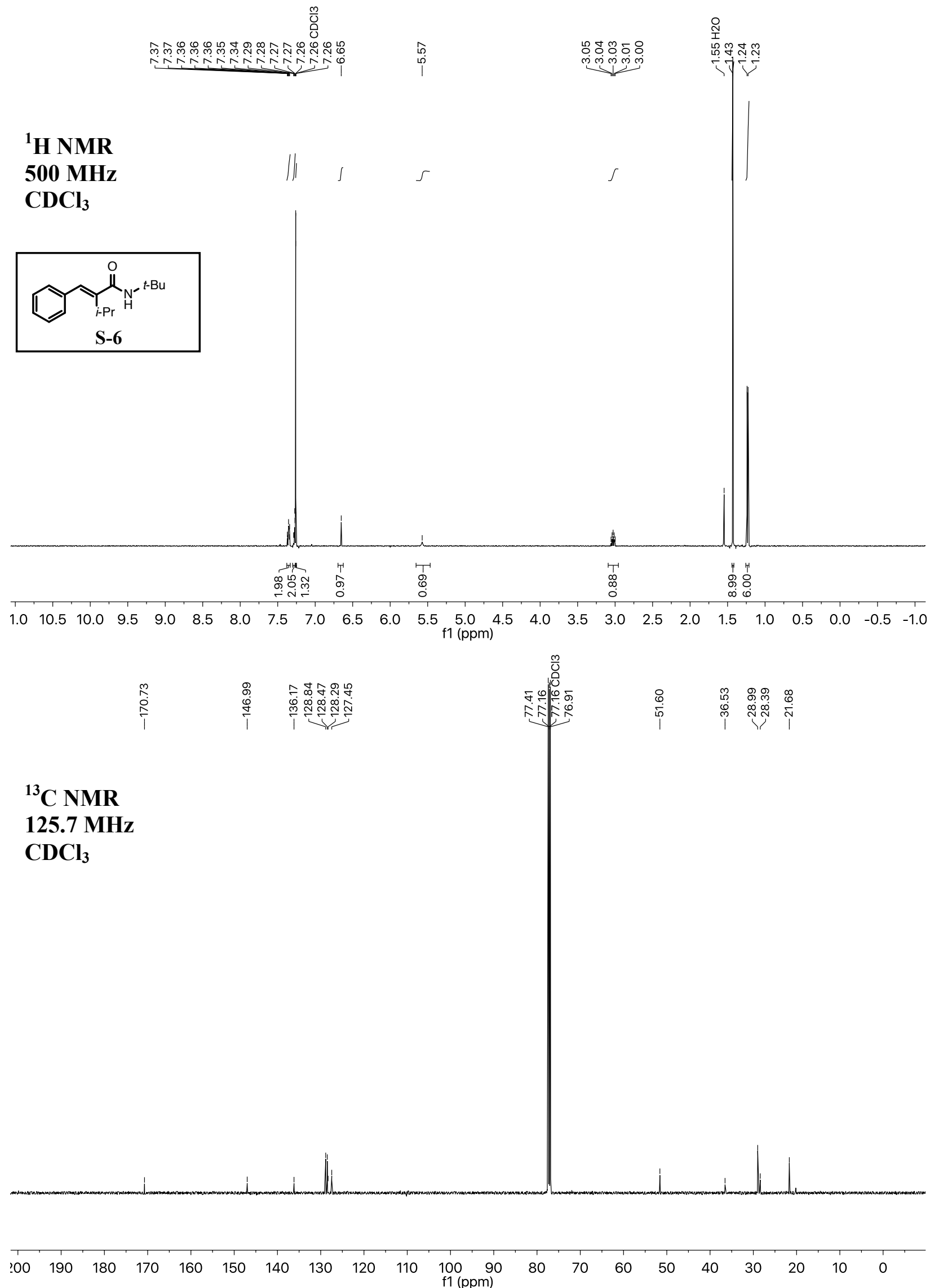


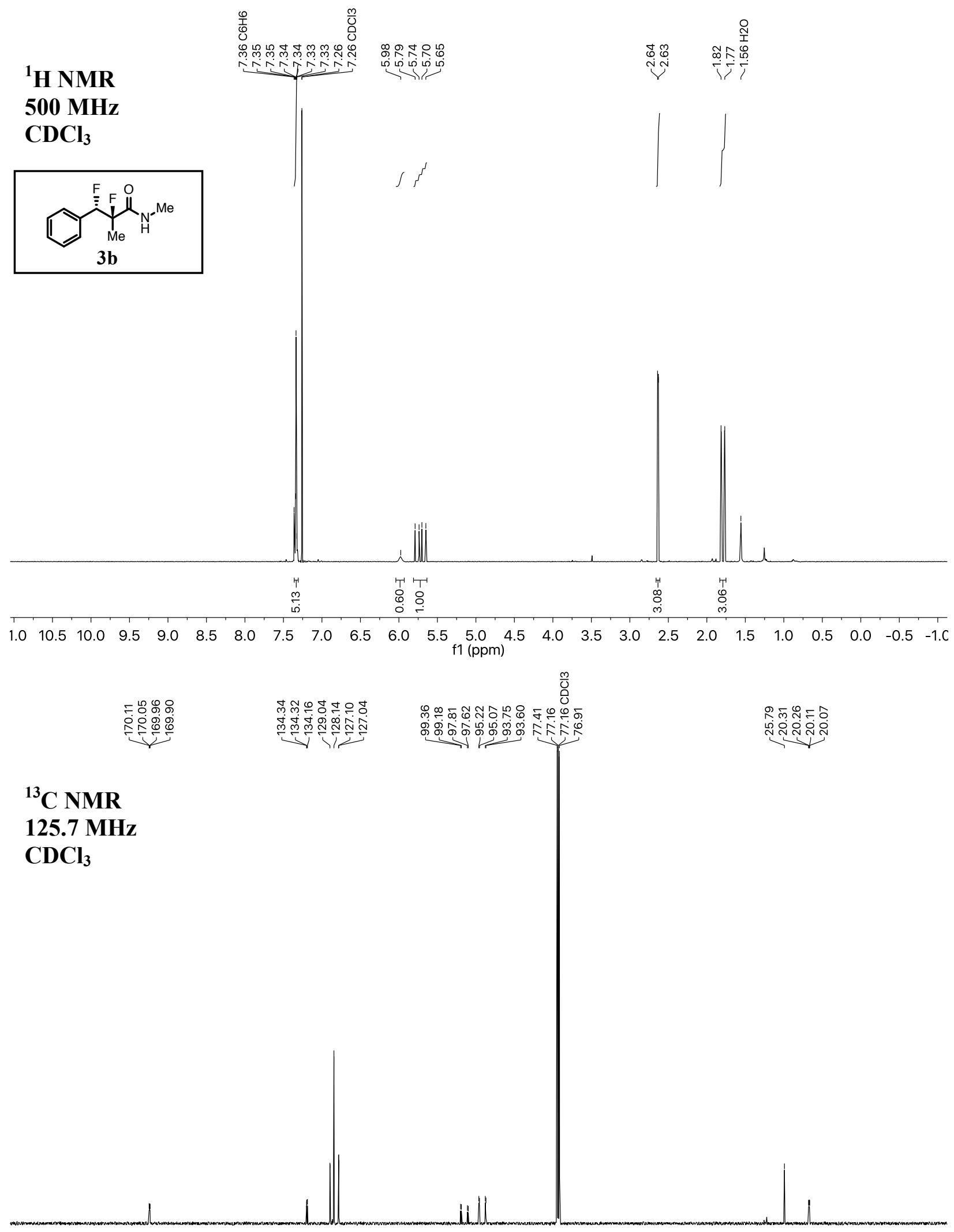

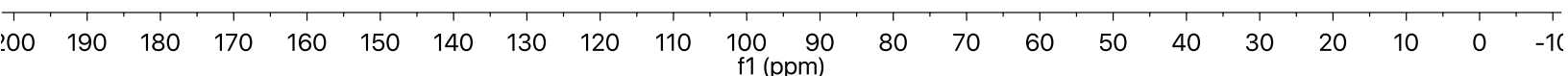




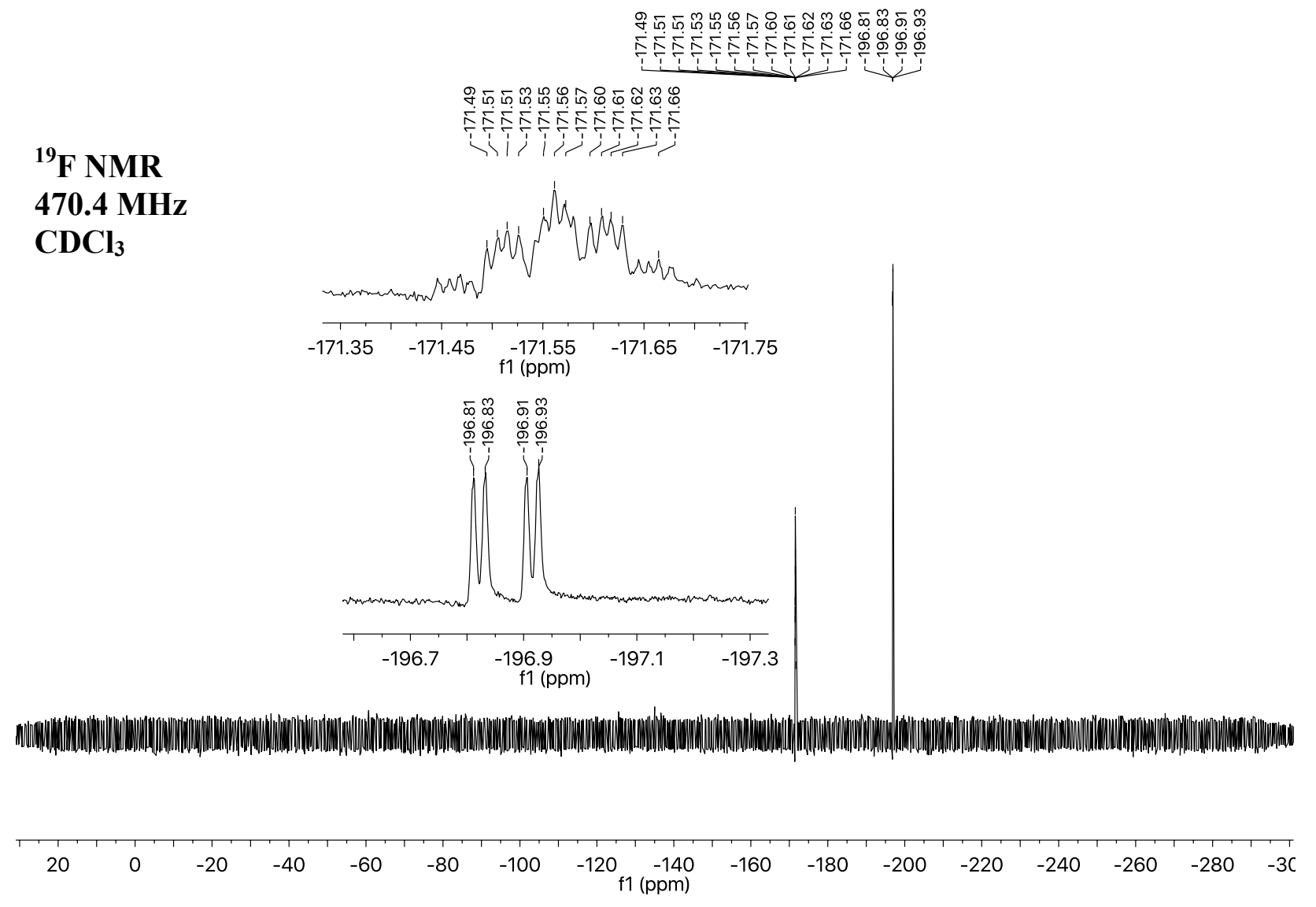



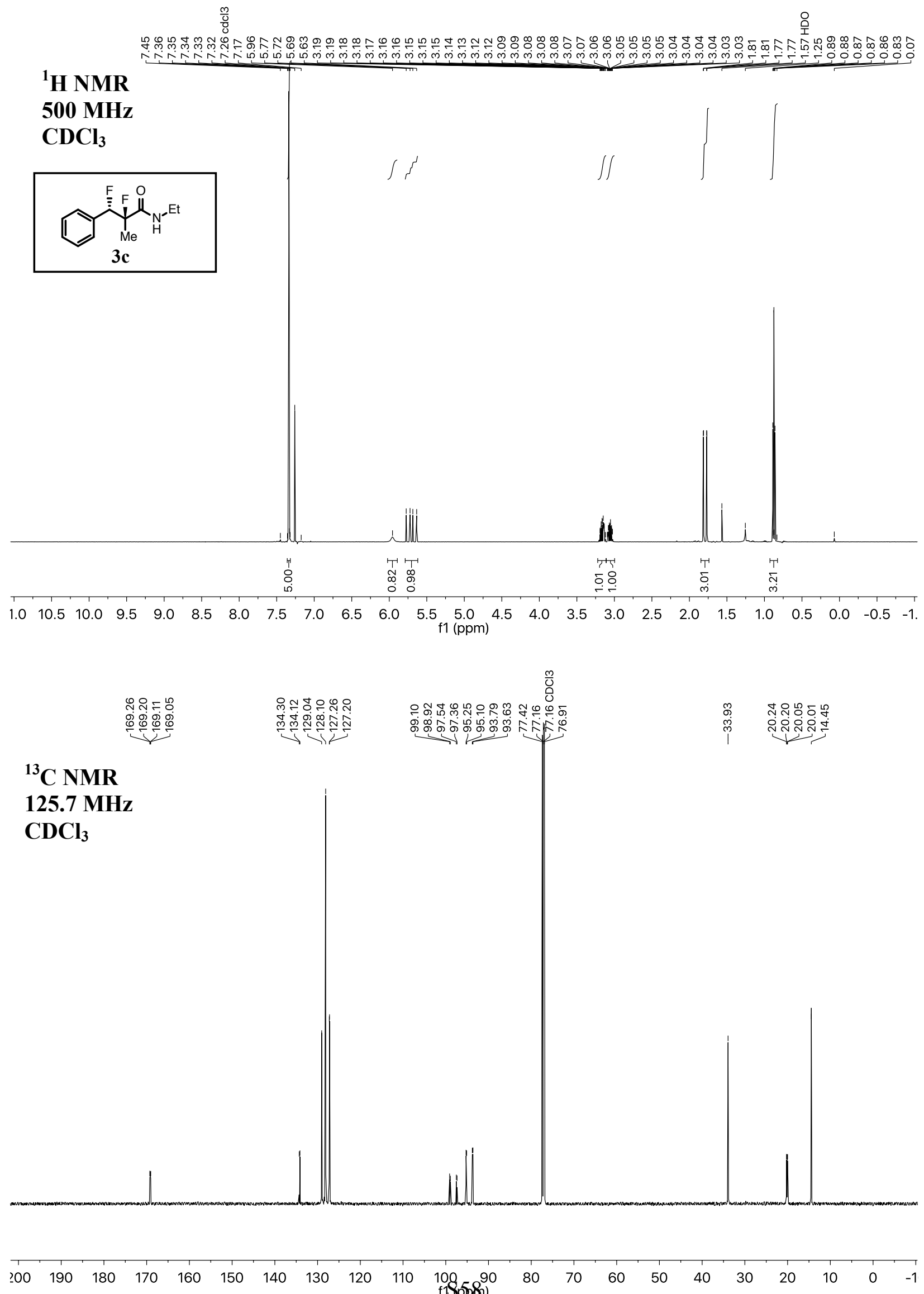


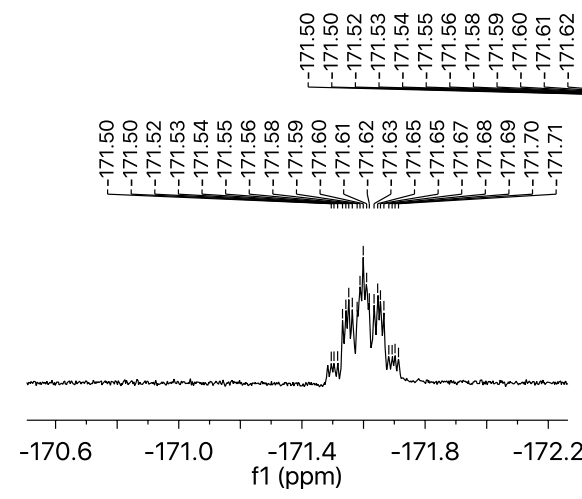

\section{${ }^{19}$ F NMR 470.4 MHz $\mathrm{CDCl}_{3}$}

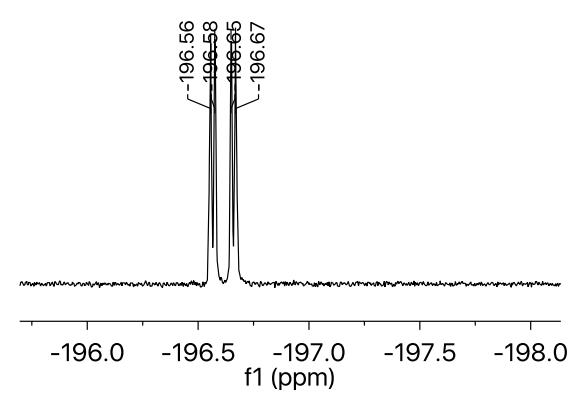

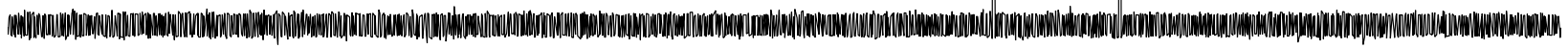

\begin{tabular}{|c|c|c|c|c|c|c|c|c|c|c|c|c|c|c|}
\hline 0 & 0 & -20 & -40 & -60 & -80 & -100 & -120 & -140 & -160 & -180 & -200 & -220 & -240 & -260 \\
\hline
\end{tabular}




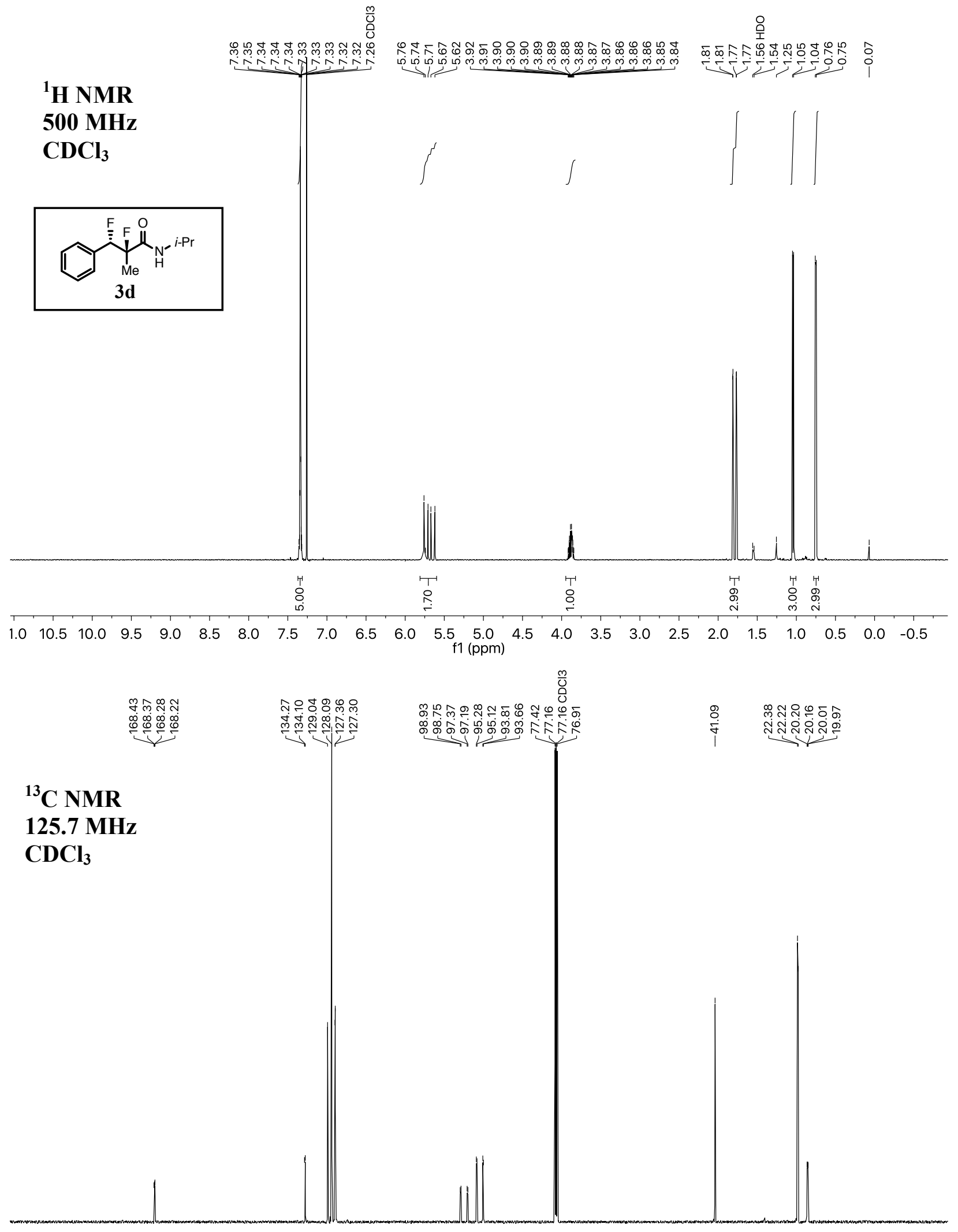

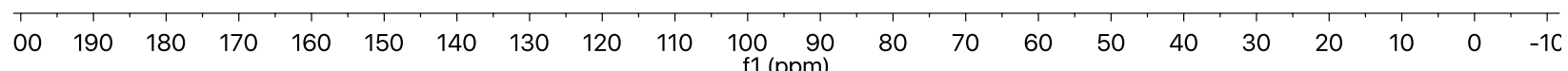



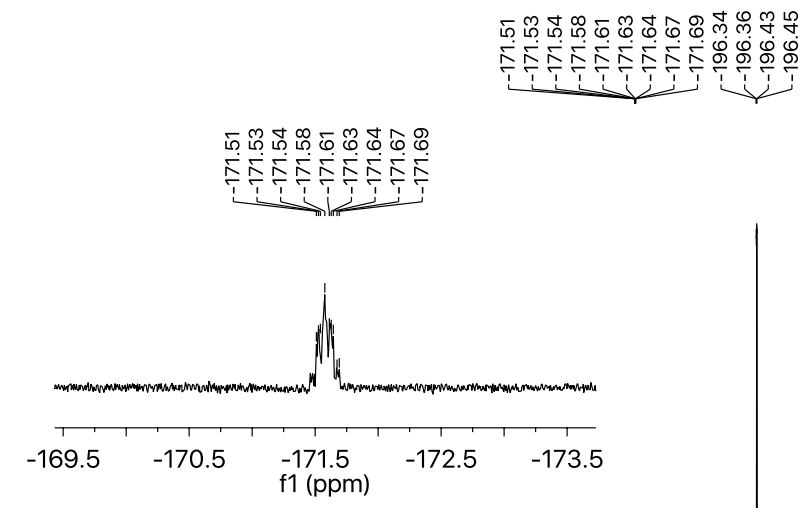

${ }^{19}$ F NMR

470.4 MHz $\mathrm{CDCl}_{3}$

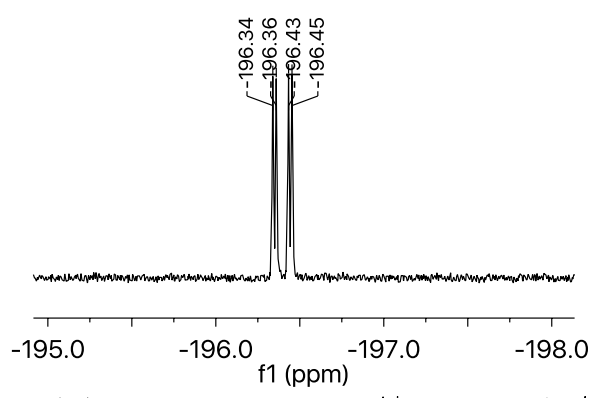

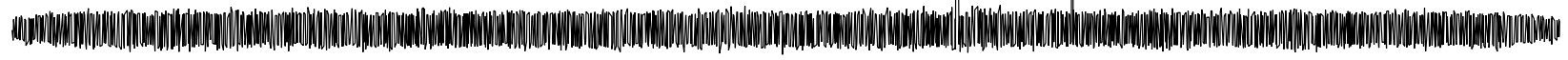

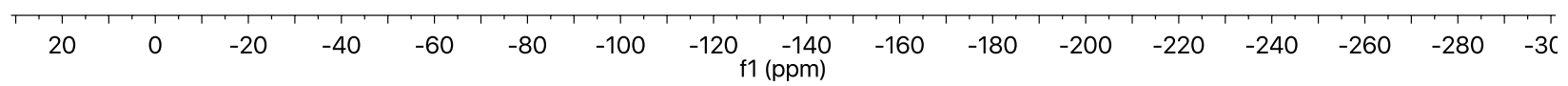



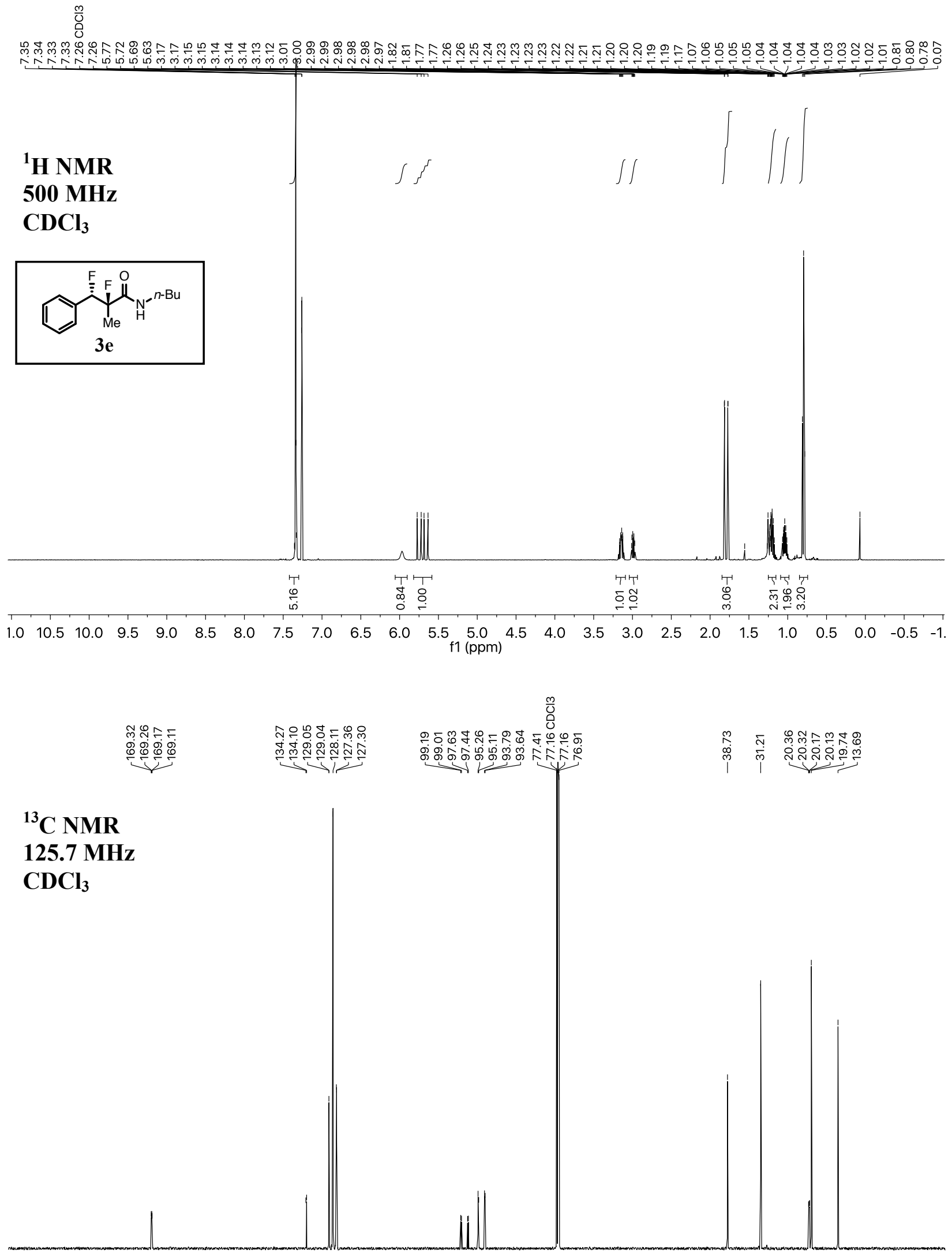

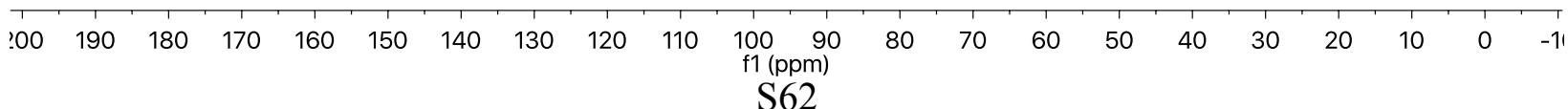




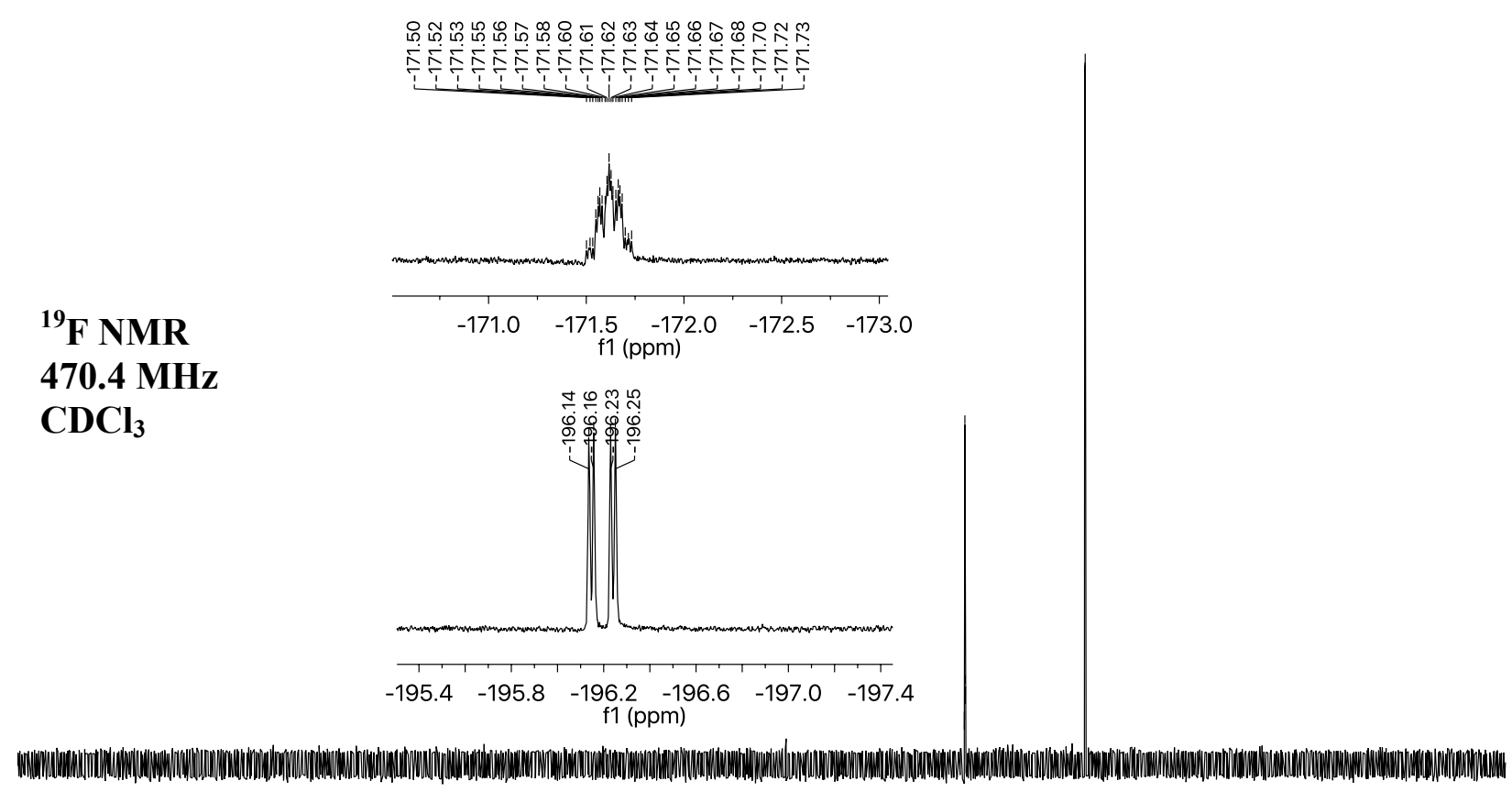

\begin{tabular}{|c|c|c|c|c|c|c|c|c|c|c|c|c|c|c|c|}
\hline 20 & $c$ & -20 & -40 & -60 & -80 & -100 & $\begin{array}{r}-120 \\
\mathrm{f} 1\end{array}$ & $\begin{array}{l}-140 \\
\mathrm{~m})\end{array}$ & -160 & -180 & -200 & -220 & -240 & -260 & -28 \\
\hline
\end{tabular}




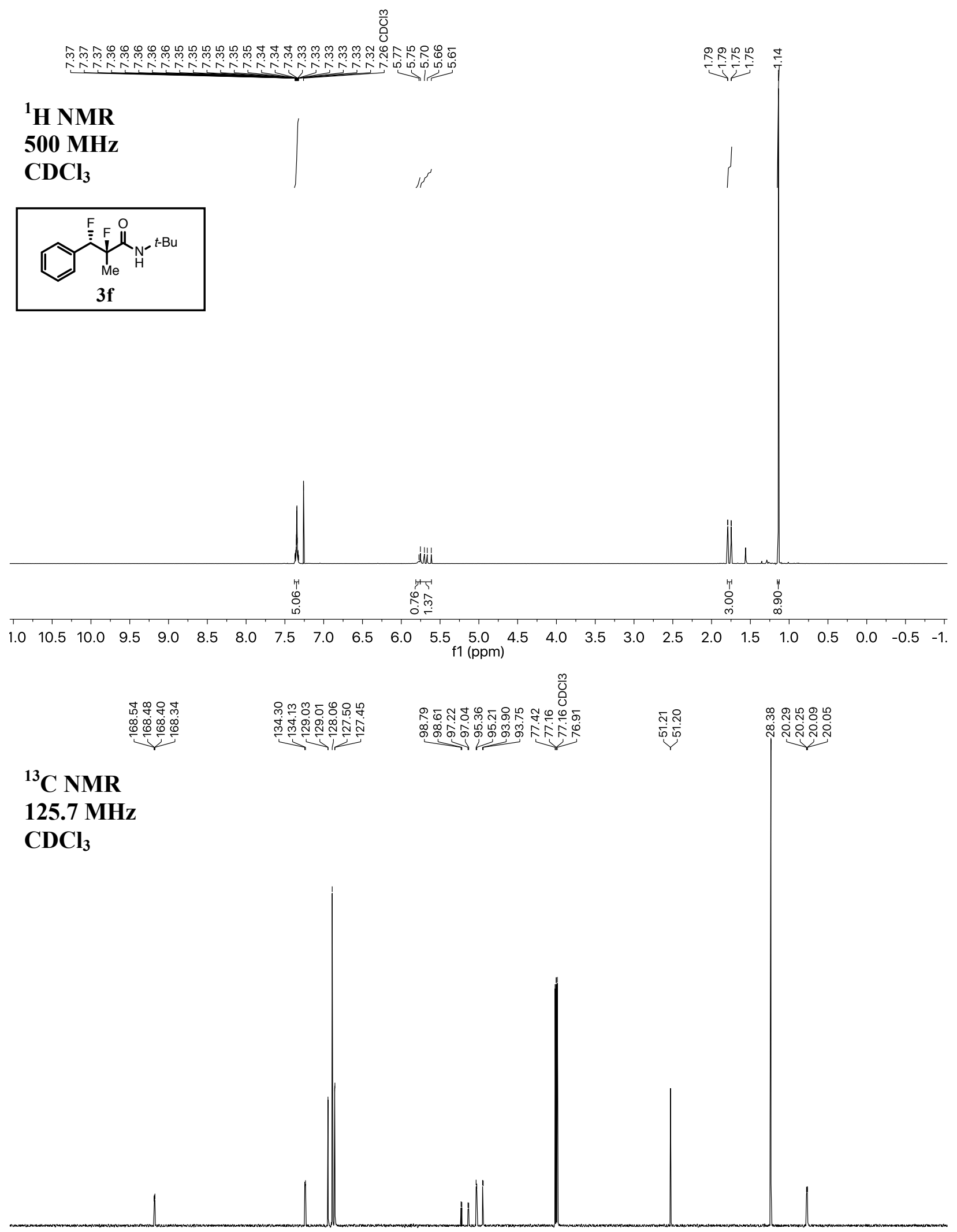

\begin{tabular}{lllllllllllllllllllll}
\hline 00 & 190 & 180 & 170 & 160 & 150 & 140 & 130 & 120 & 110 & $\underset{f 1}{100}(\mathrm{ppm})$ & 80 & 70 & 60 & 50 & 40 & 30 & 20 & 10 & 0 & $-1 \mathrm{C}$
\end{tabular} 


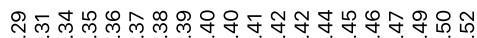

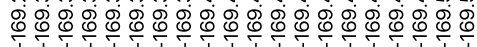

${ }^{19}$ F NMR

470.4 MHz

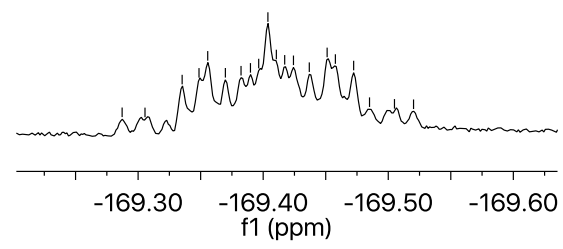

$\mathrm{CDCl}_{3}$

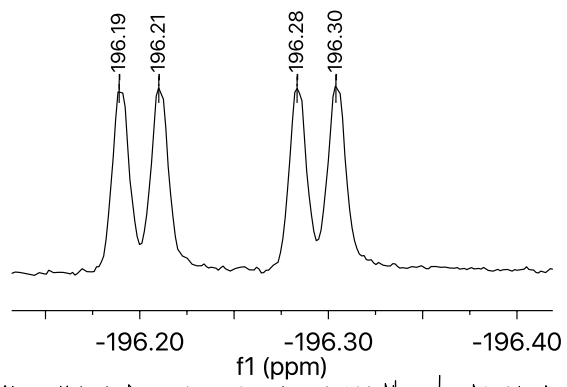

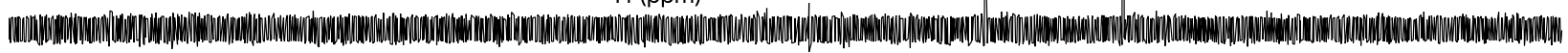

\begin{tabular}{|c|c|c|c|c|c|c|c|c|c|c|c|c|c|c|}
\hline ?0 & 0 & -20 & -40 & -60 & -80 & -100 & -120 & -140 & -160 & -180 & -200 & -220 & -240 & -260 \\
\hline
\end{tabular}


${ }^{1}$ H NMR

$500 \mathrm{MHz}$

$\mathrm{CDCl}_{3}$
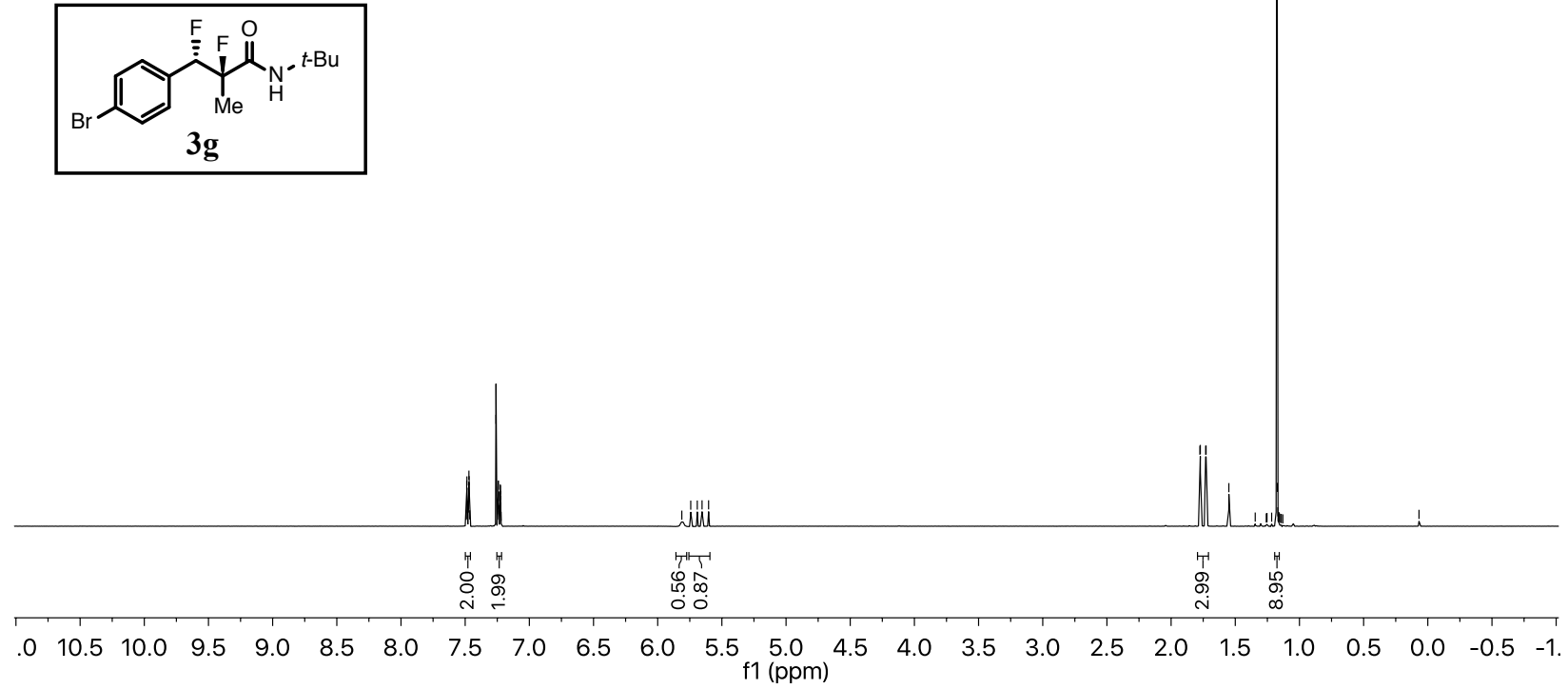

\section{ชุก ำ ำ}

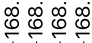

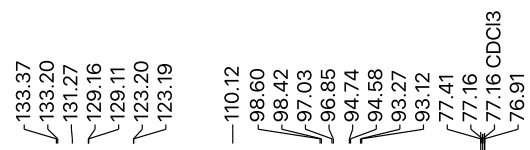

\section{${ }^{13} \mathrm{C}$ NMR \\ 125.7 MHz \\ $\mathrm{CDCl}_{3}$}
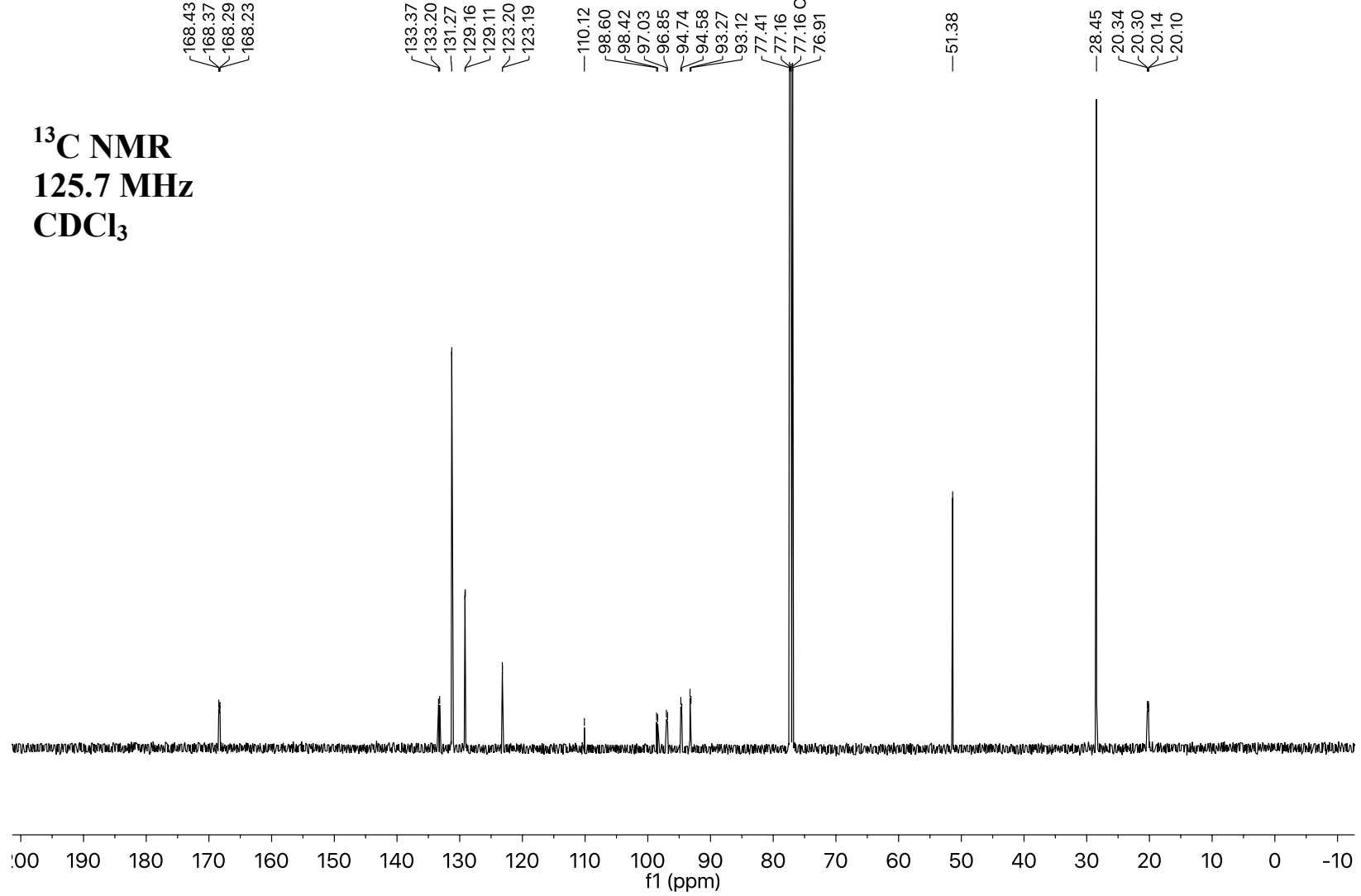


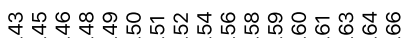

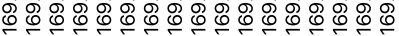
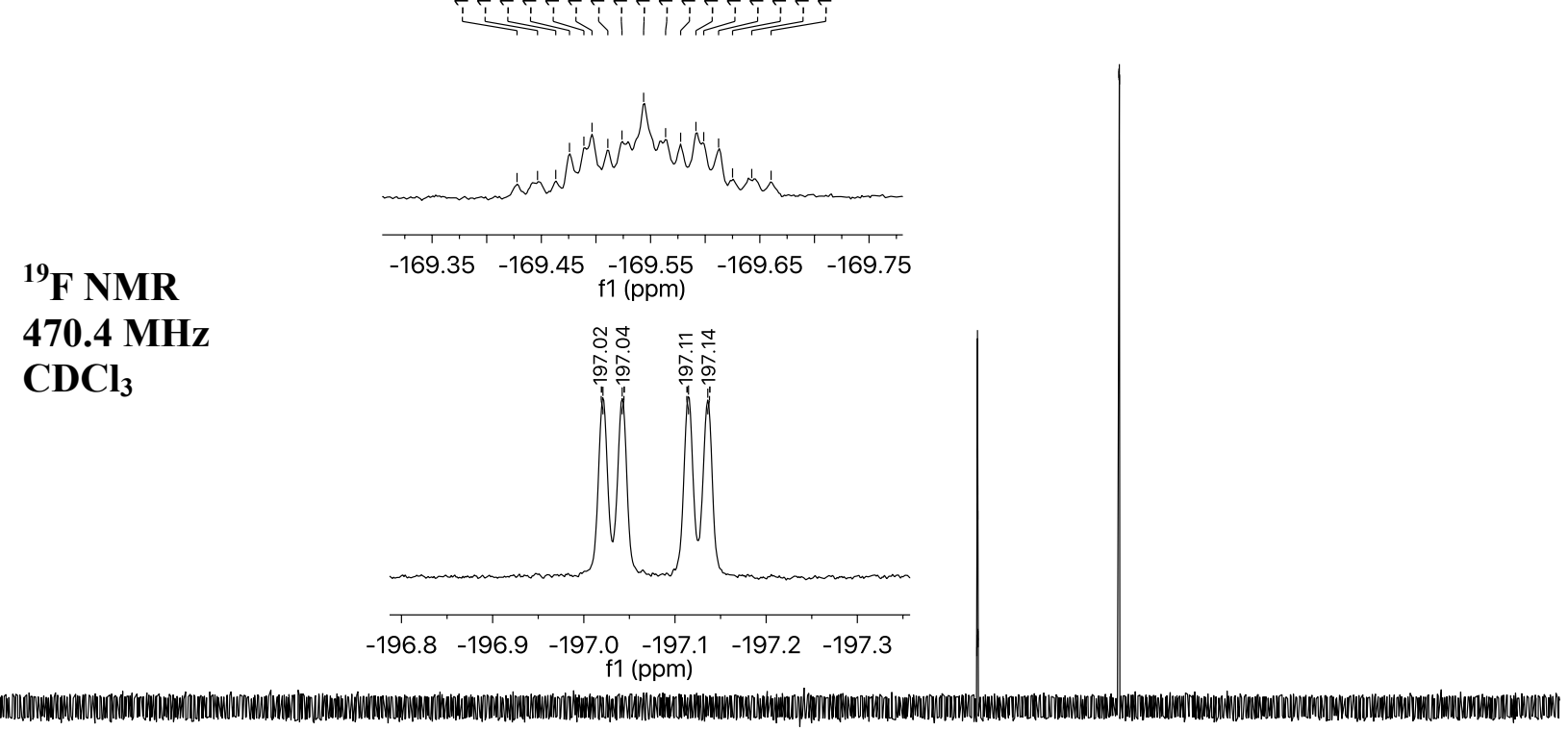

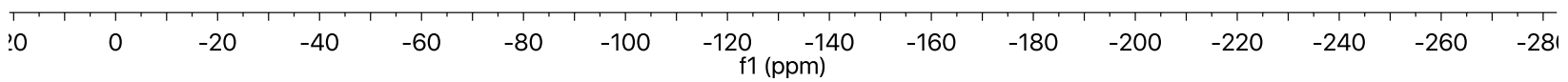



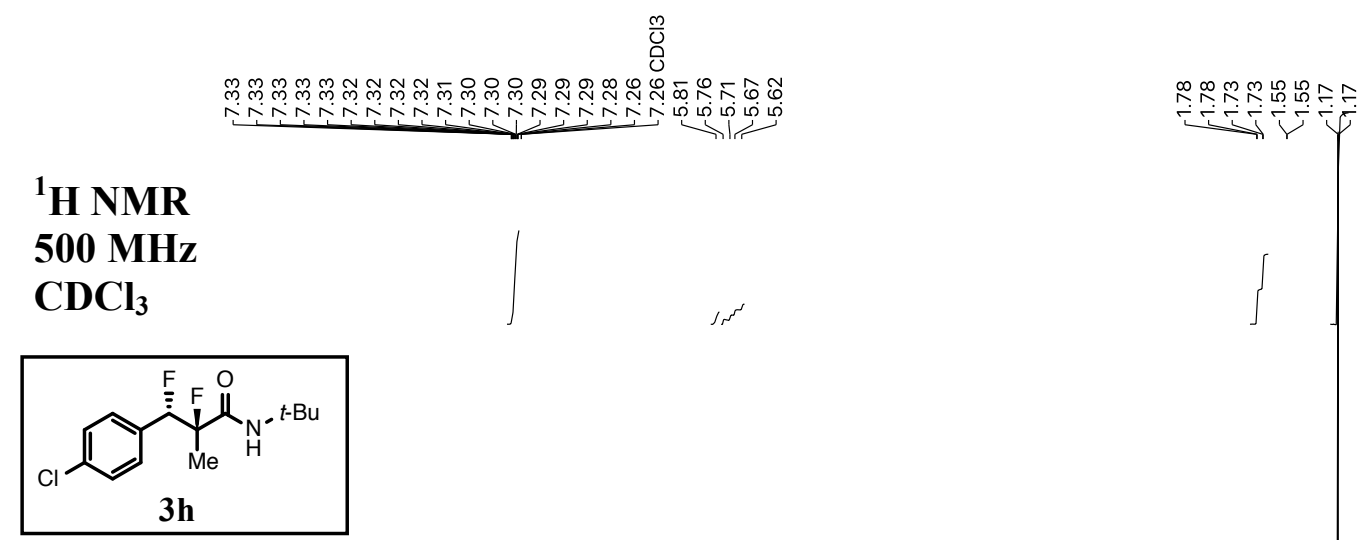

${ }^{1}$ H NMR

$500 \mathrm{MHz}$

$\mathrm{CDCl}_{3}$
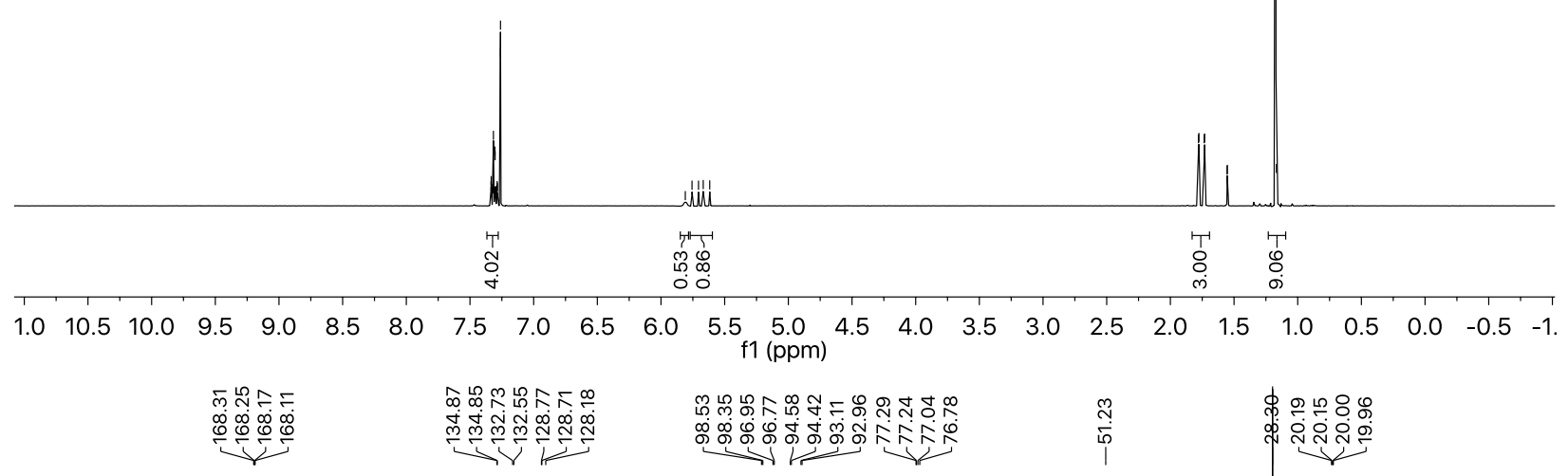

\section{${ }^{13} \mathrm{C}$ NMR \\ 125.7 MHz \\ $\mathrm{CDCl}_{3}$}

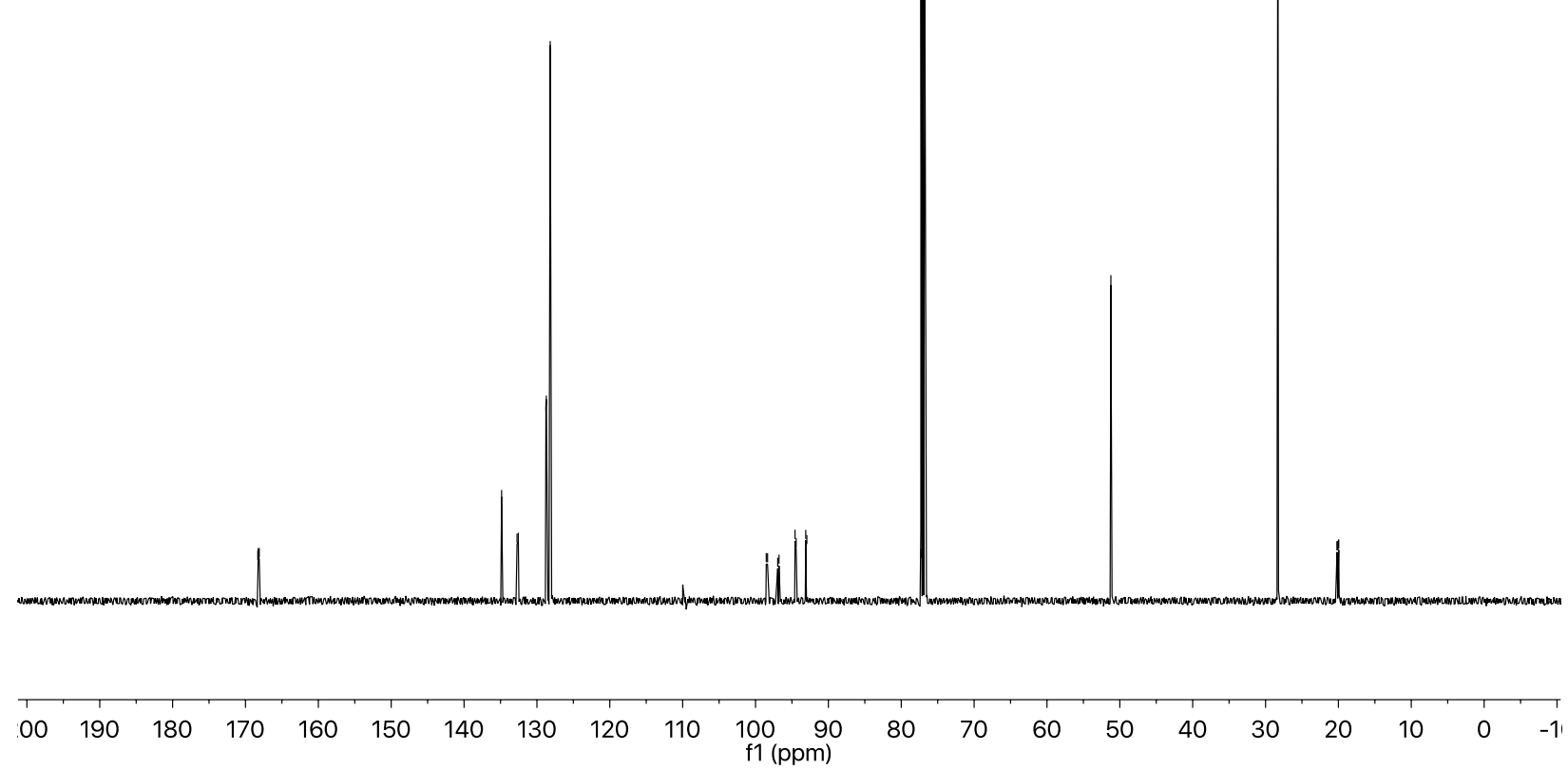




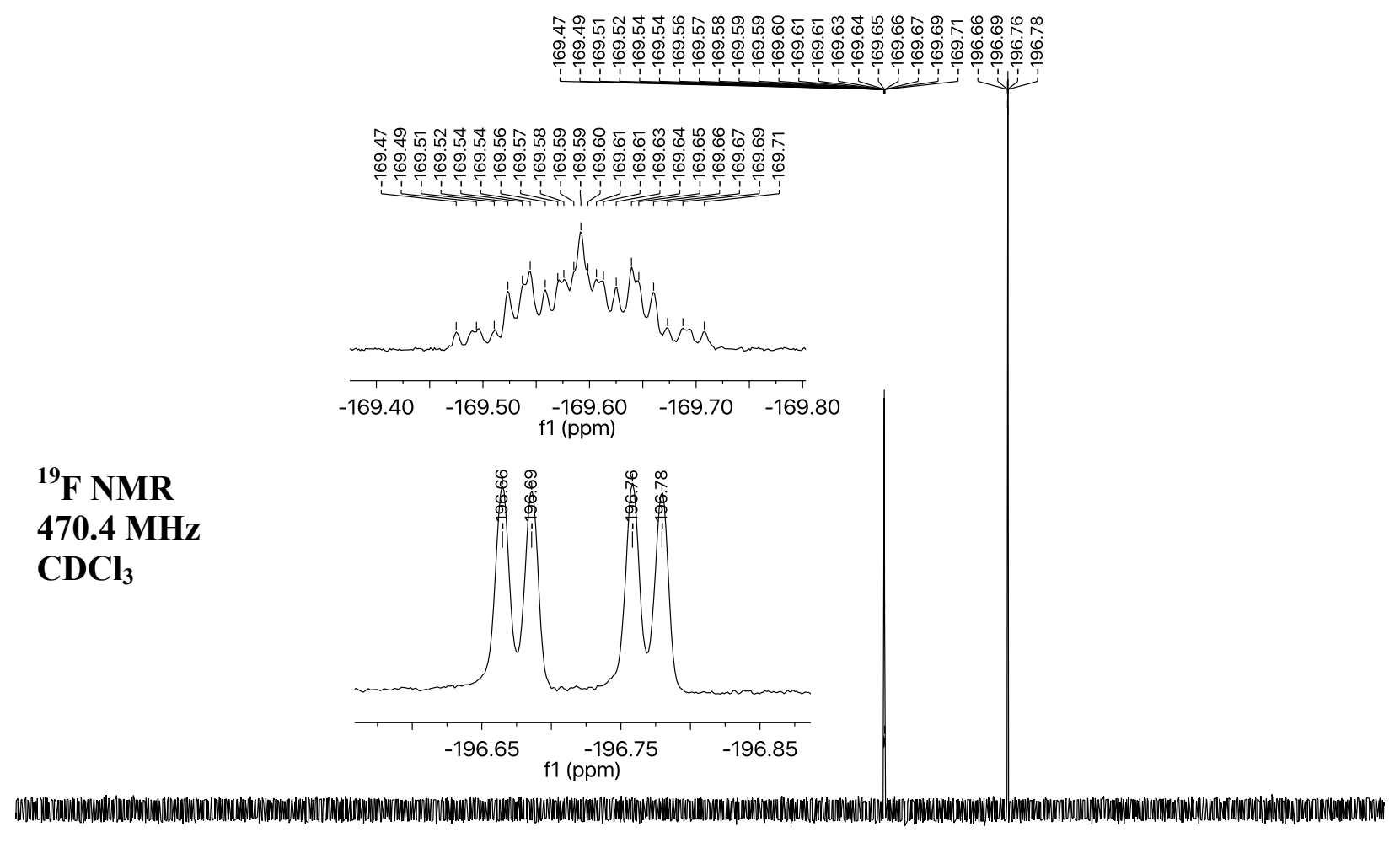

\begin{tabular}{|c|c|c|c|c|c|c|c|c|c|c|c|c|c|c|}
\hline 10 & -10 & -30 & -50 & -70 & -90 & -110 & $\begin{array}{c}-130 \\
\mathrm{f1}(\mathrm{ppm})\end{array}$ & -150 & -170 & -190 & -210 & -230 & -250 & -270 \\
\hline
\end{tabular}


${ }^{1}$ H NMR

$500 \mathrm{MHz}$

$\mathrm{CDCl}_{3}$
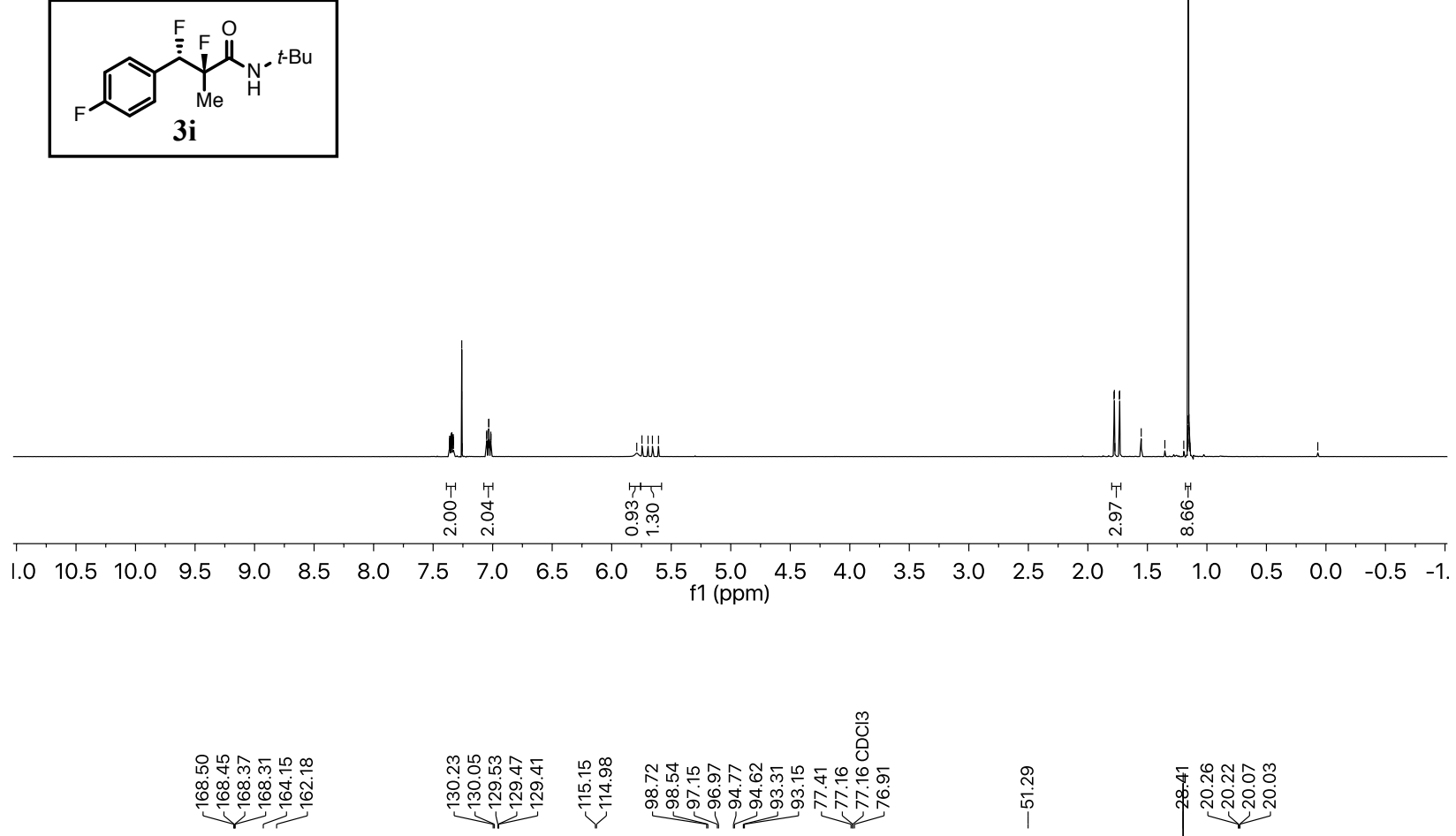

\section{บ
0
0}

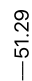

×

๙ ํํㅇํํ

\section{${ }^{13}$ C NMR \\ 125.7 MHz \\ $\mathrm{CDCl}_{3}$}

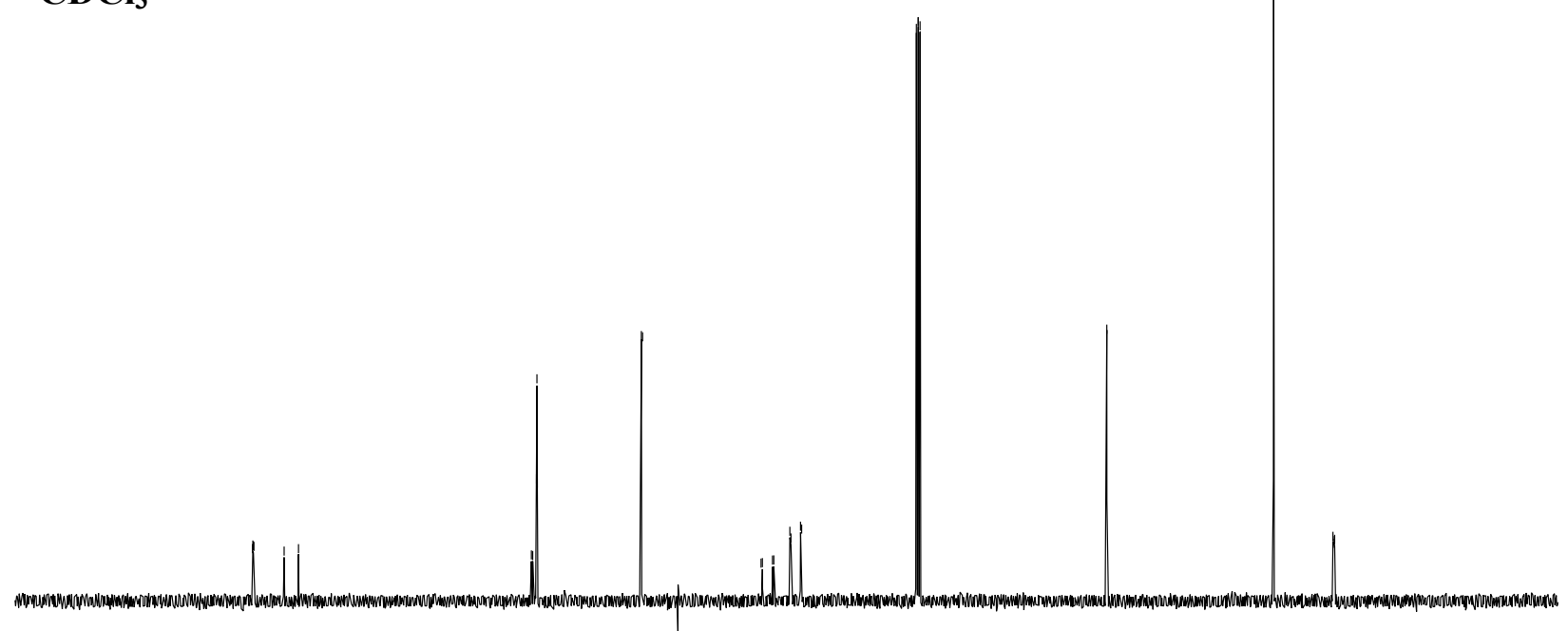




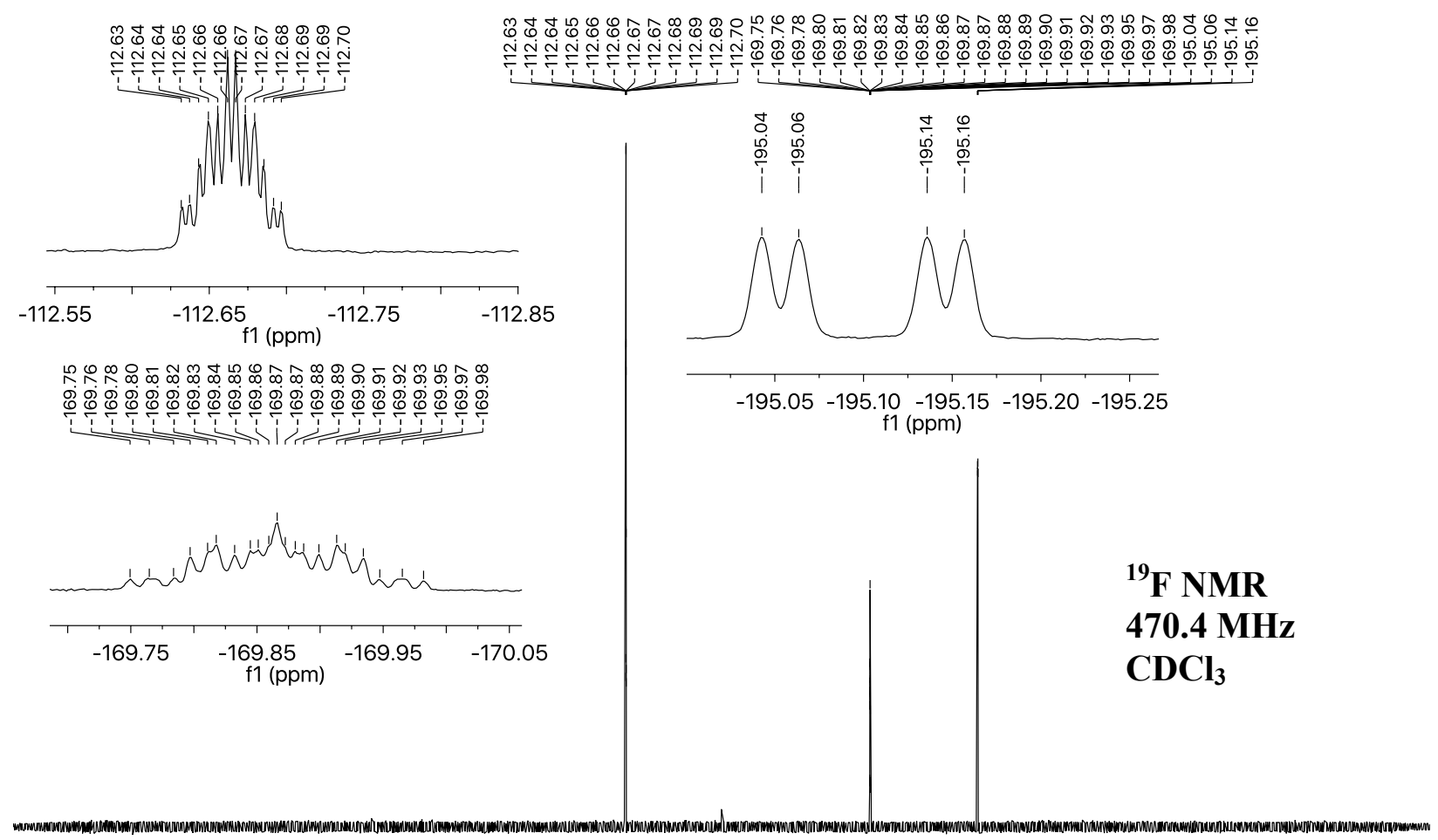

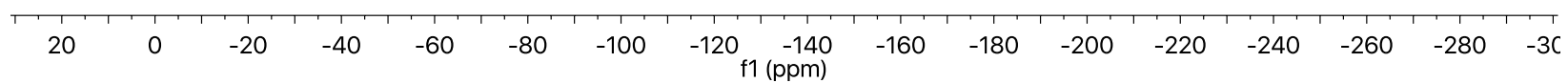




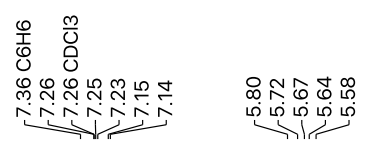

\section{${ }^{1}$ H NMR \\ $500 \mathrm{MHz}$ \\ $\mathrm{CDCl}_{3}$}
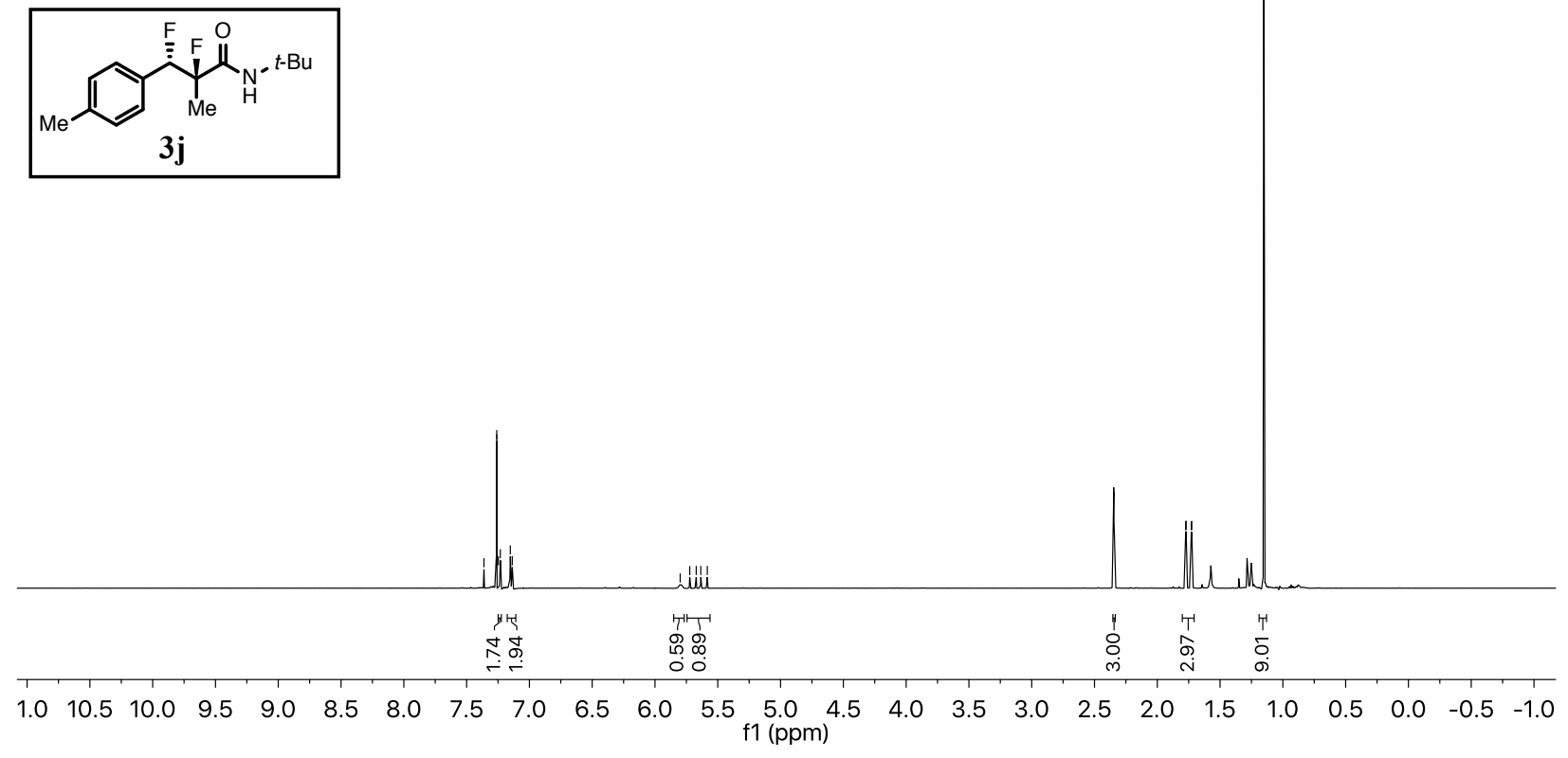

ธิธธยูำ

$\begin{array}{ll}\infty & 0 \\ 0 & 0 \\ 0 & 0\end{array}$

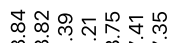

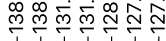

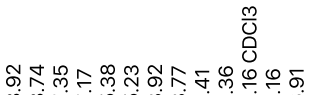

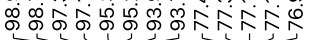

\section{${ }^{13}$ C NMR \\ 125.7 MHz \\ $\mathrm{CDCl}_{3}$}

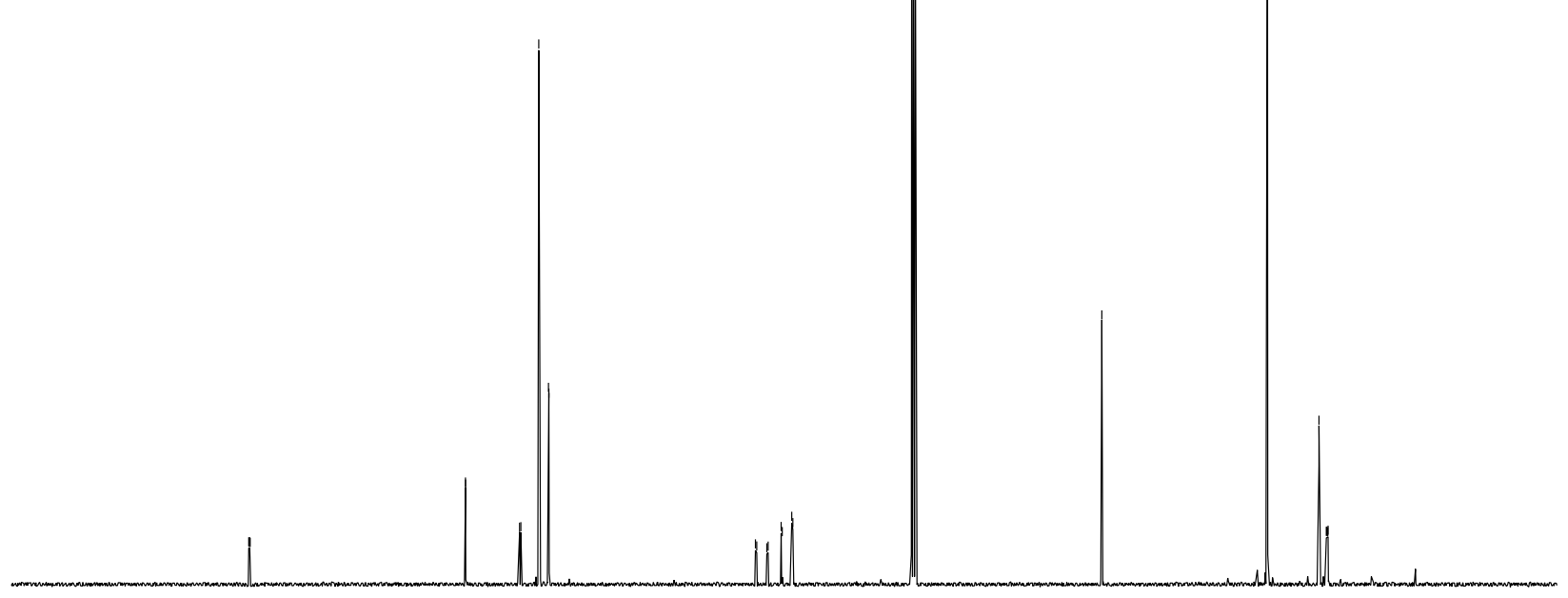

$\begin{array}{llllllllllllllllllllll}00 & 190 & 180 & 170 & 160 & 150 & 140 & 130 & 120 & 110 & 100 & 90 & 80 & 70 & 60 & 50 & 40 & 30 & 20 & 10 & 0 & -10\end{array}$ 


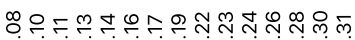

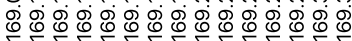

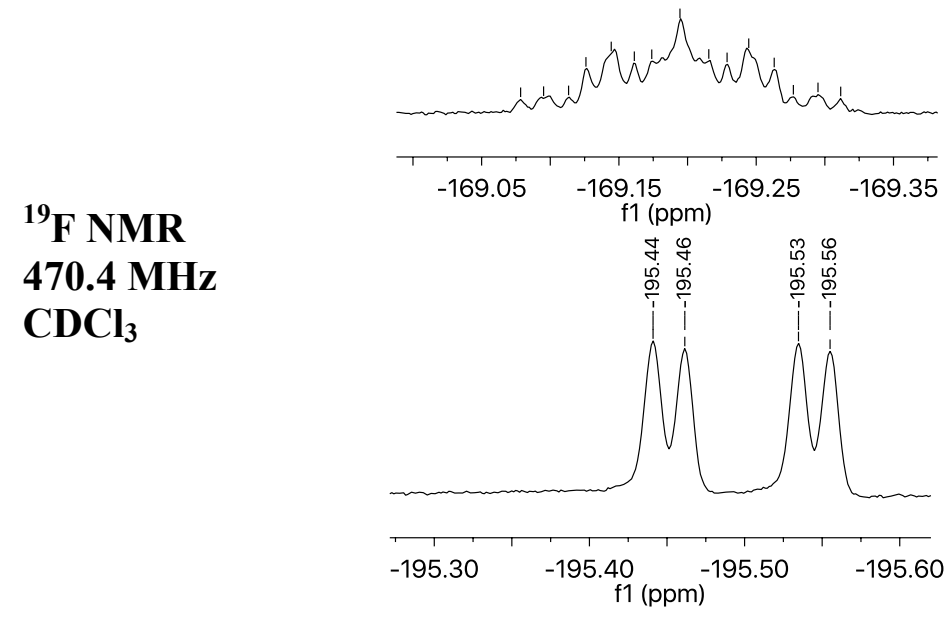

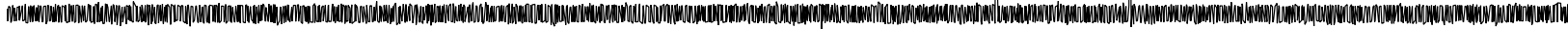

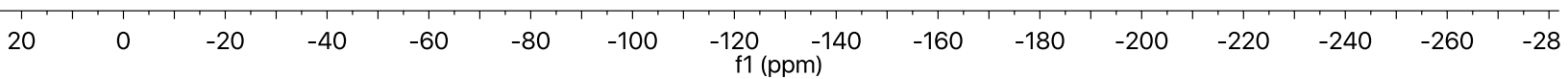



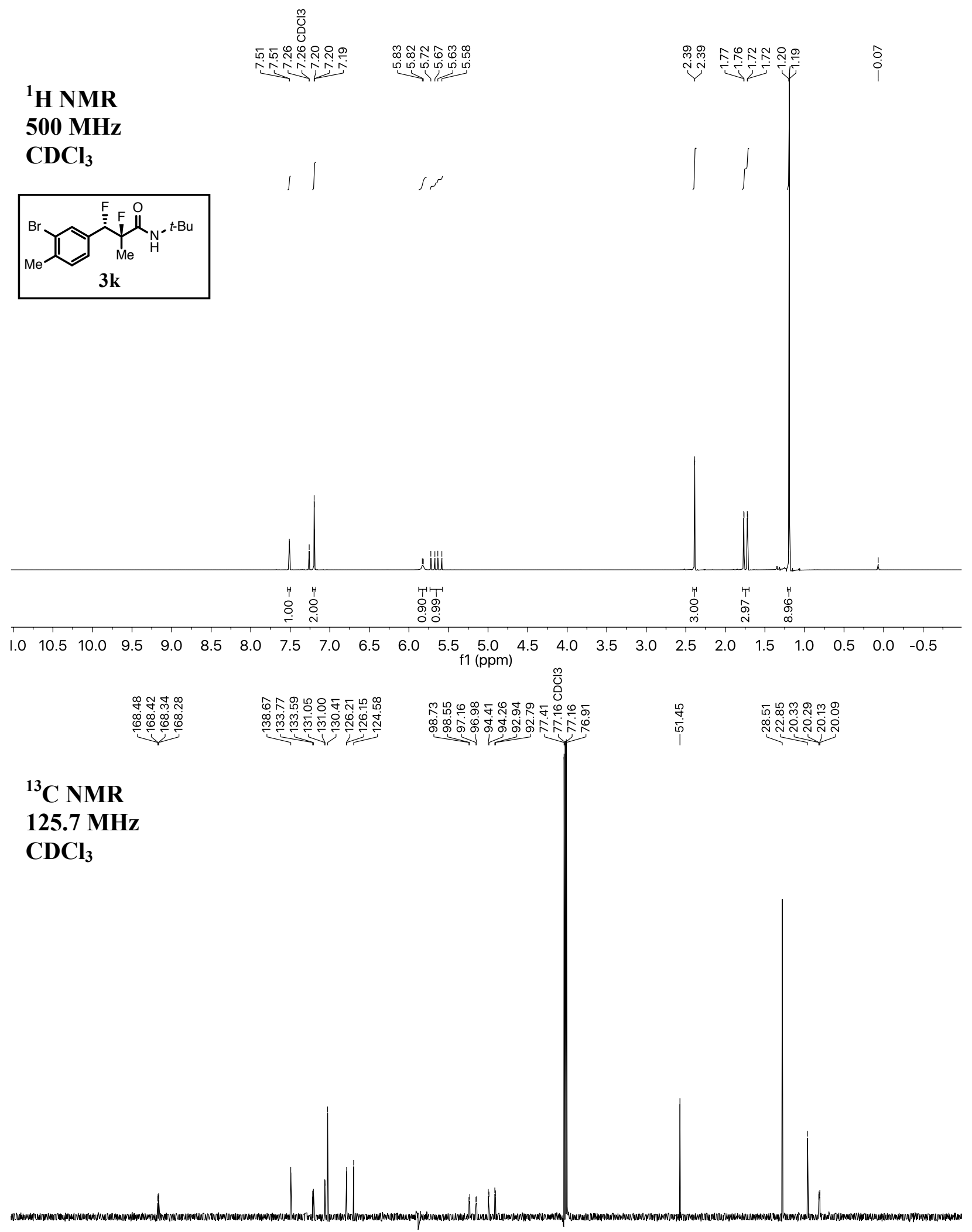

.00

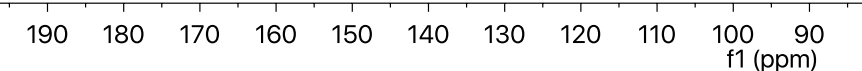

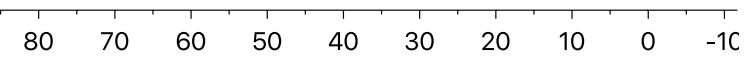




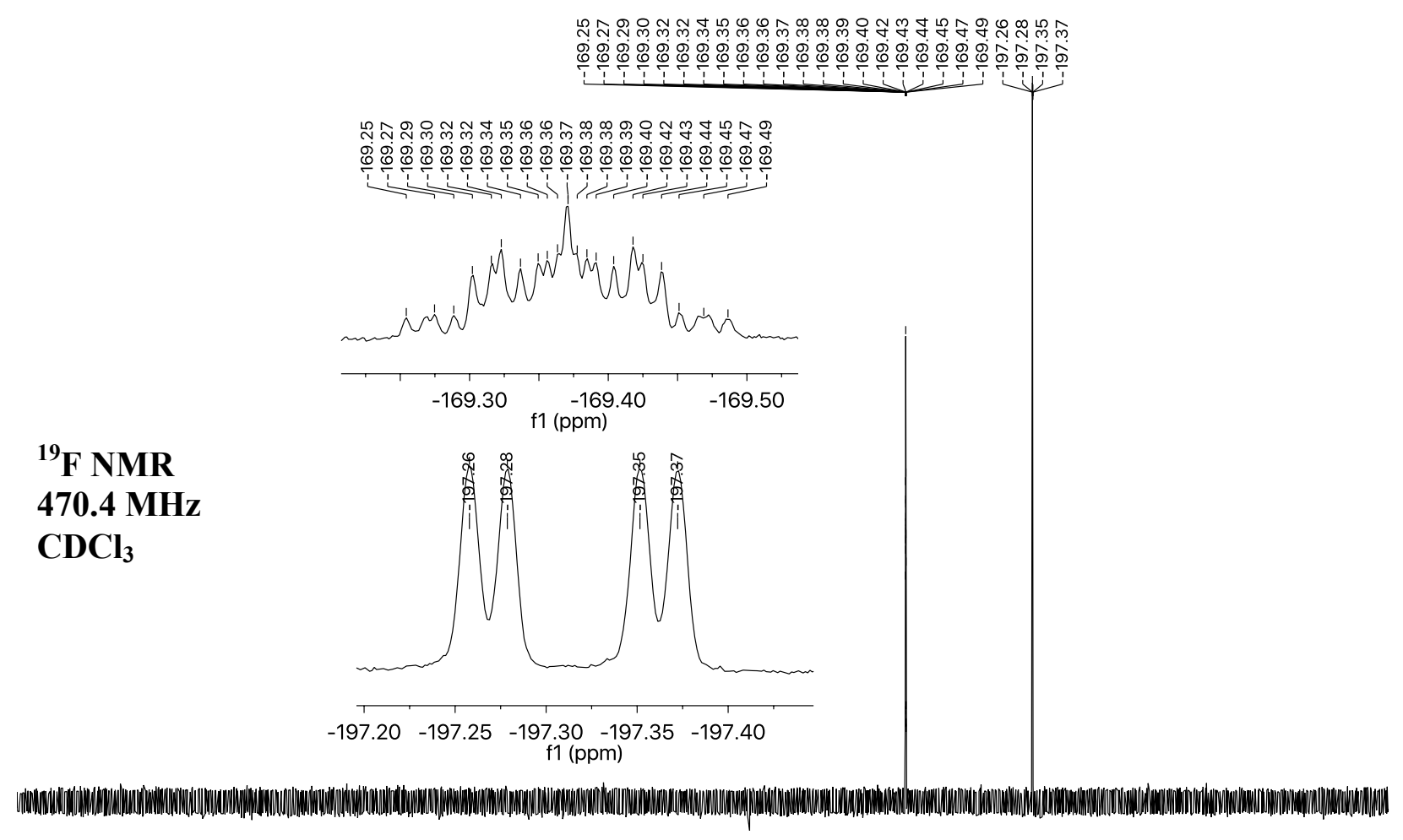

\begin{tabular}{|c|c|c|c|c|c|c|c|c|c|c|c|c|c|c|}
\hline 1 & 1 & 1 & 1 & 1 & 1 & 1 & 1 & 1 & $T$ & 11 & 1 & T & 1 & 1 \\
\hline 10 & -10 & -30 & -50 & -70 & -90 & -110 & $\begin{array}{c}-130 \\
f 1(p p m)\end{array}$ & -150 & -170 & -190 & -210 & -230 & -250 & -270 \\
\hline
\end{tabular}



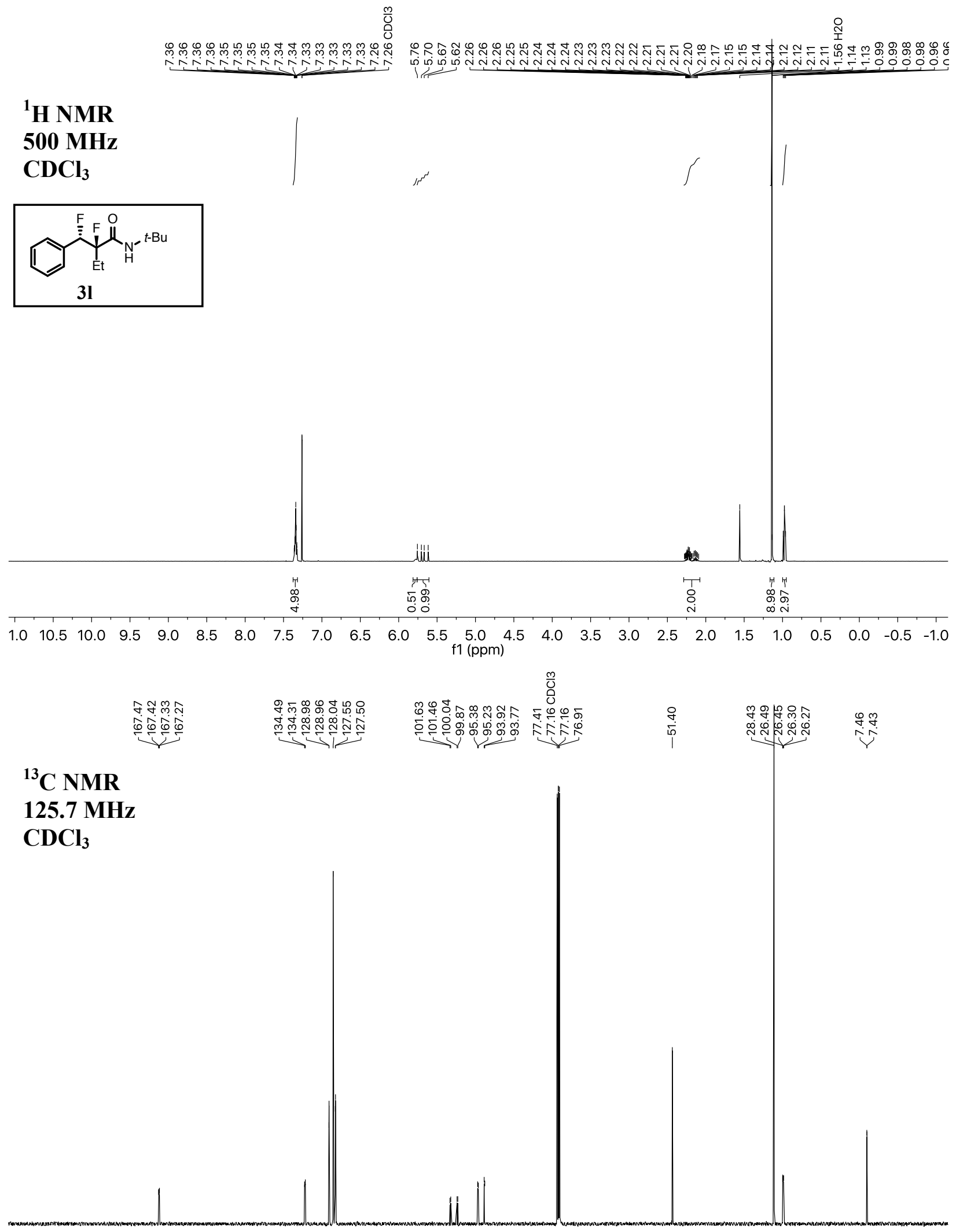

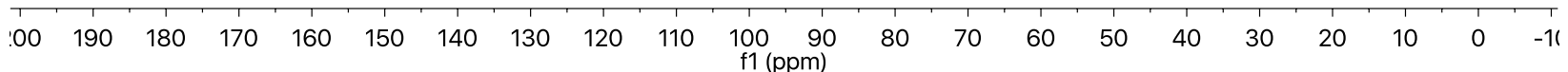




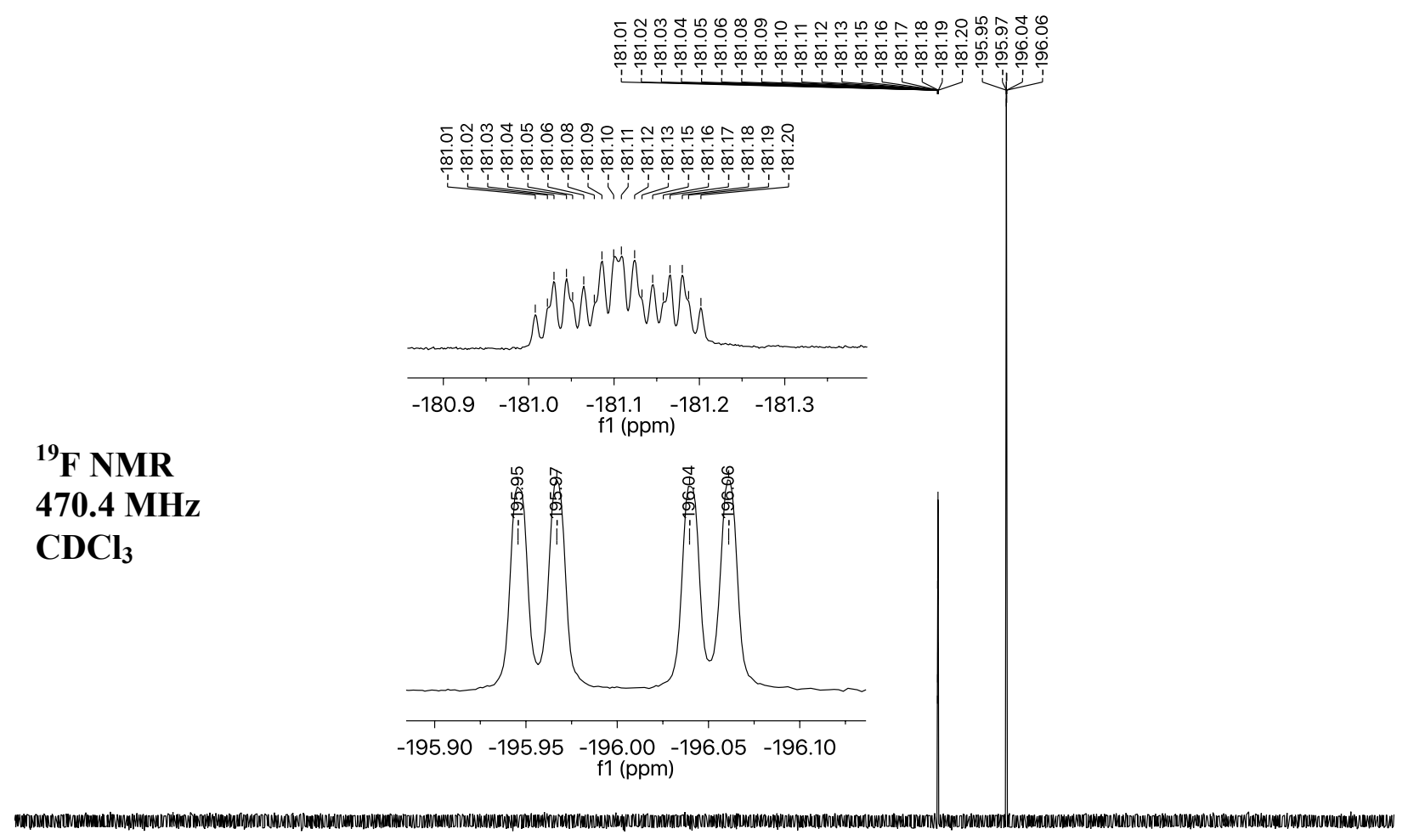

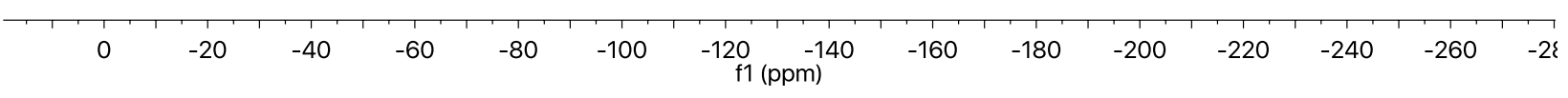




\section{${ }^{1}$ H NMR}

$500 \mathrm{MHz}$

$\mathrm{CDCl}_{3}$

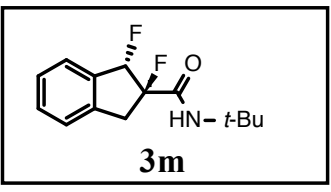




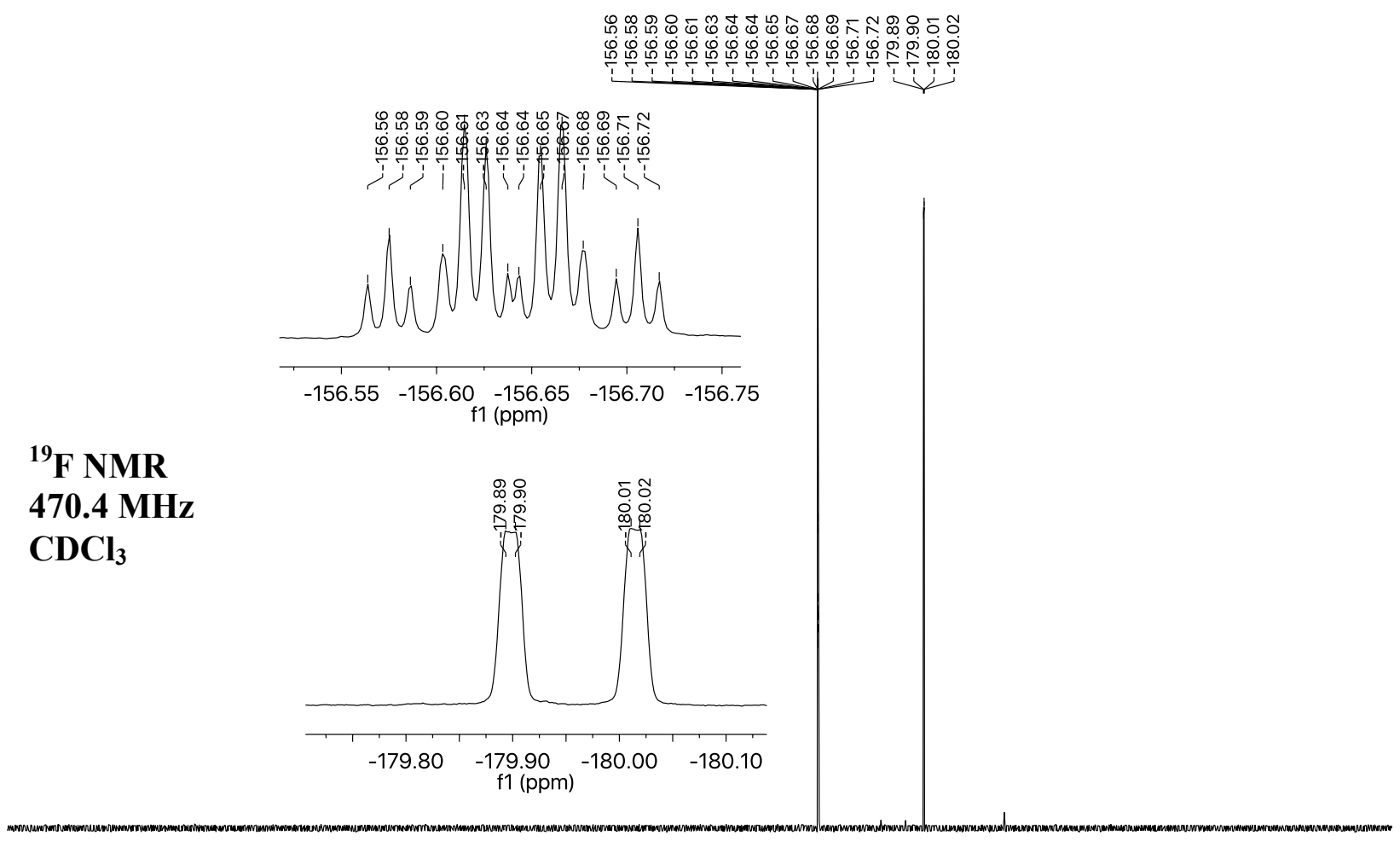

\section{${ }^{19}$ F NMR 470.4 MHz $\mathrm{CDCl}_{3}$}

\begin{tabular}{|c|c|c|c|c|c|c|c|c|c|c|c|c|c|c|c|}
\hline$T$ & 1 & 1 & 1 & 1 & 1 & 1 & 1. & 11 & 1 & 1 & 1 & 1 & 1. & 1 & $T$ \\
\hline$? 0$ & 0 & -20 & -40 & -60 & -80 & -100 & $\begin{array}{r}-120 \\
\text { f1 }\end{array}$ & $\begin{array}{l}-140 \\
m)\end{array}$ & -160 & -180 & -200 & -220 & -240 & -260 & -281 \\
\hline
\end{tabular}



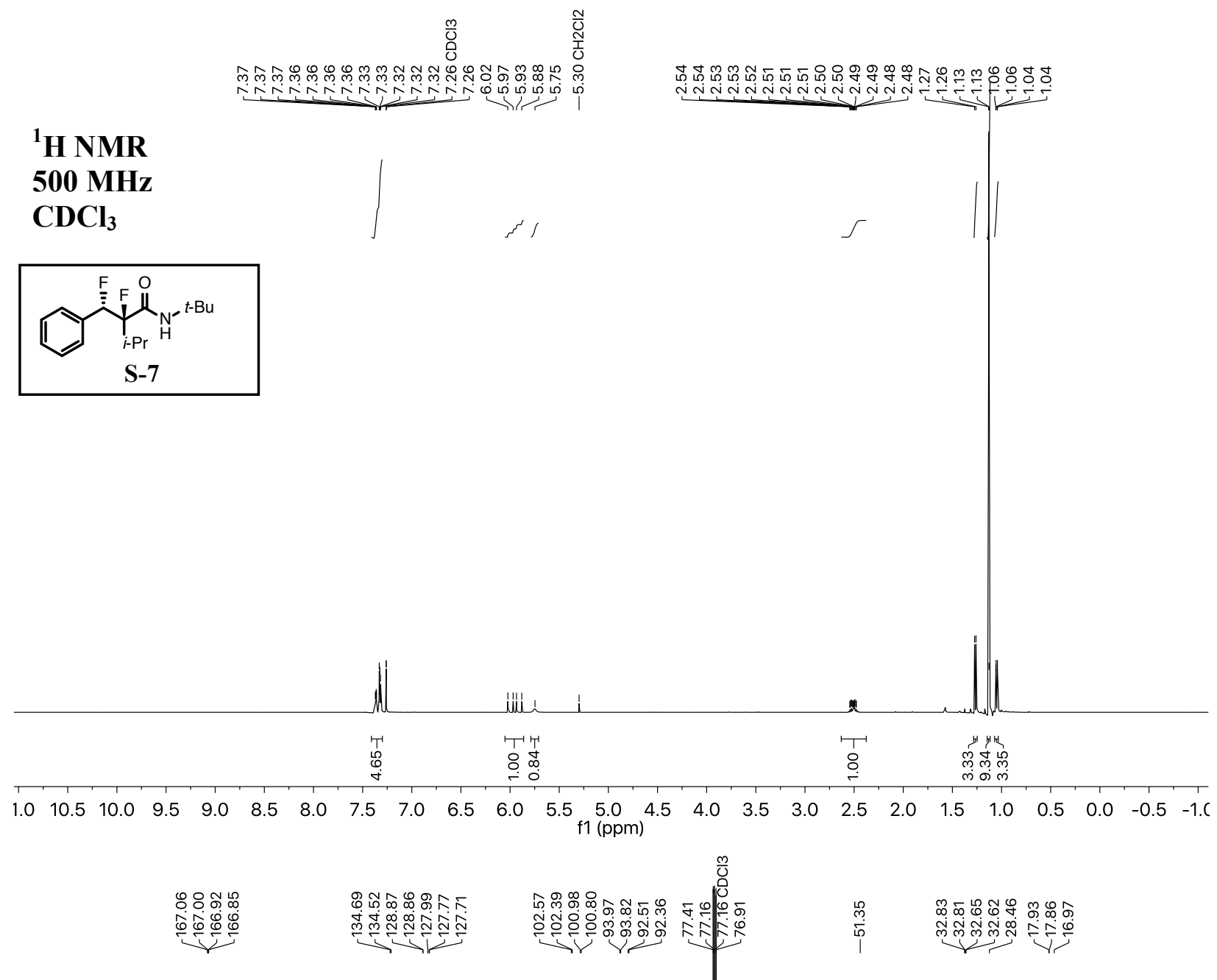

\section{${ }^{13}$ C NMR \\ 125.7 MHz \\ $\mathrm{CDCl}_{3}$}

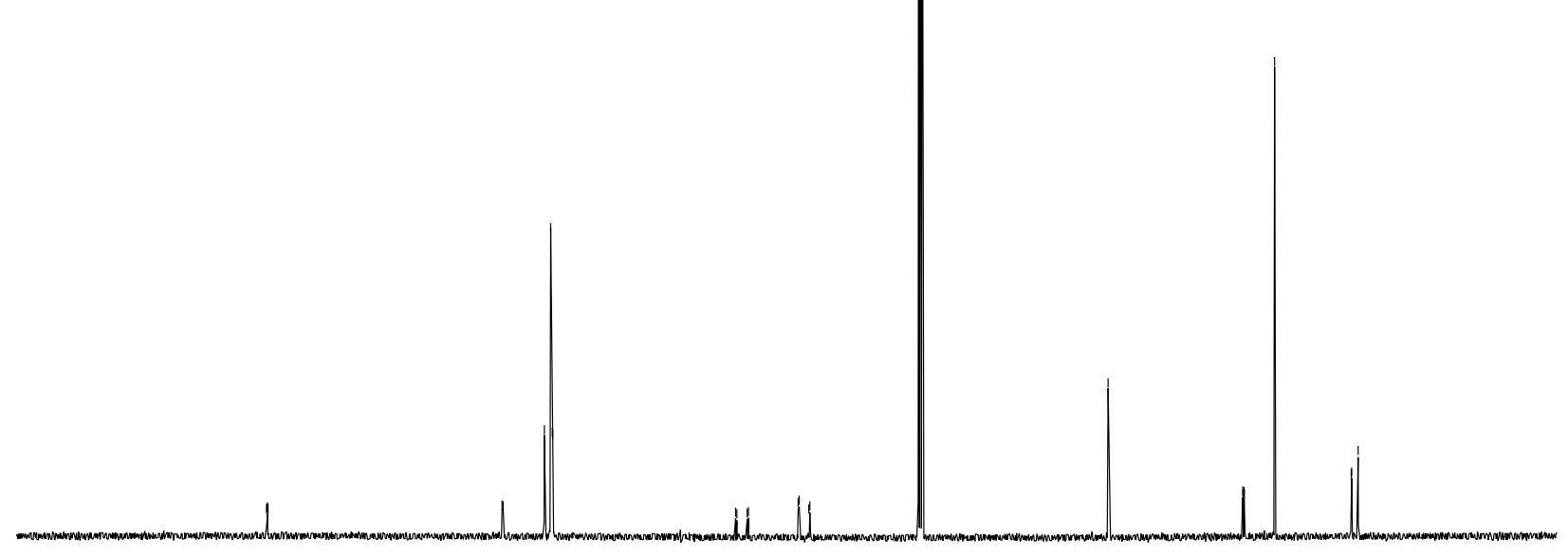

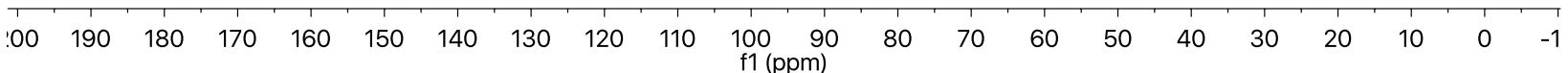




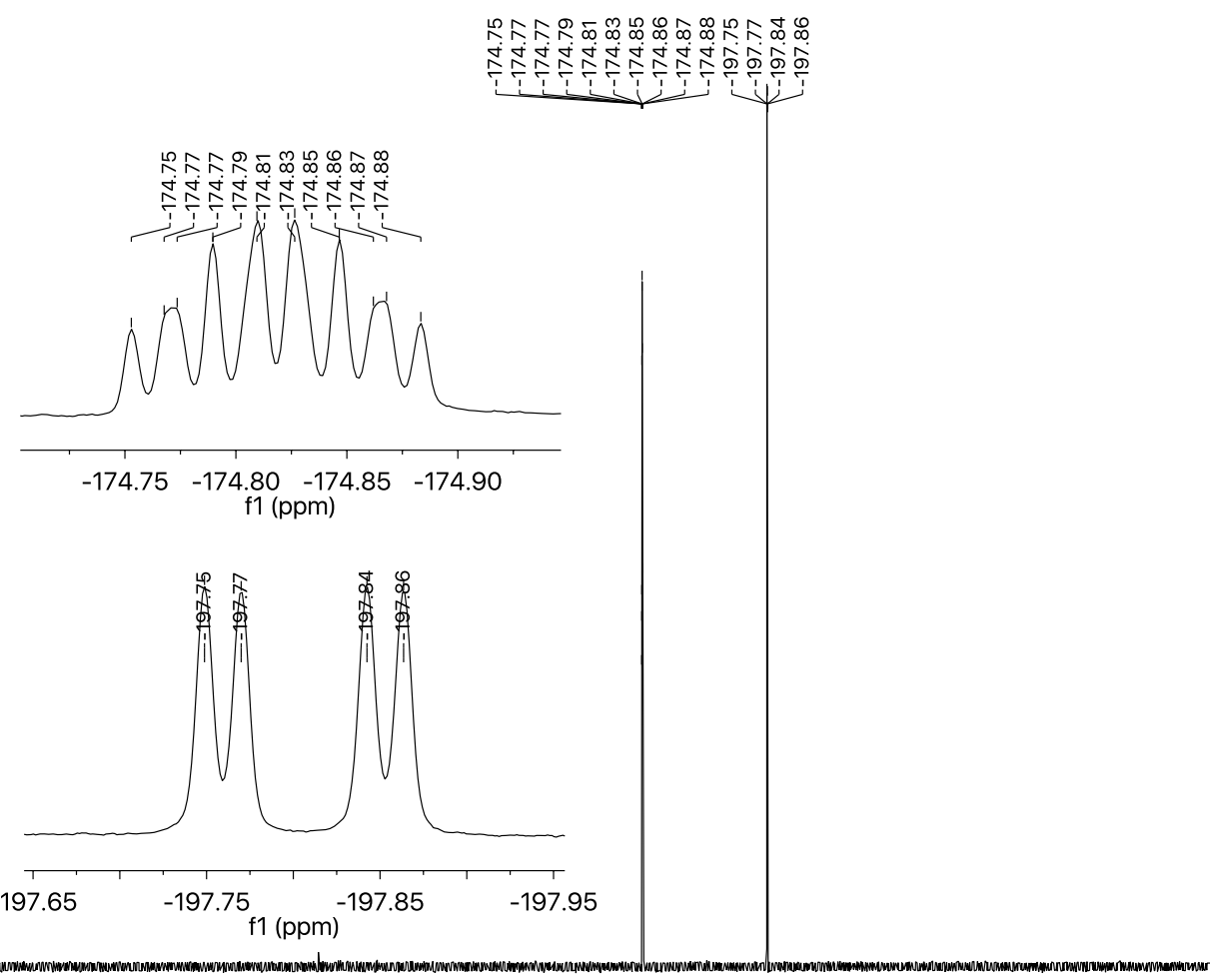

\begin{tabular}{|c|c|c|c|c|c|c|c|c|c|c|c|c|c|c|}
\hline 10 & -10 & -30 & -50 & -70 & -90 & -110 & $\begin{array}{c}-130 \\
\mathrm{f1}(\mathrm{ppm})\end{array}$ & -150 & -170 & -190 & -210 & -230 & -250 & -270 \\
\hline
\end{tabular}




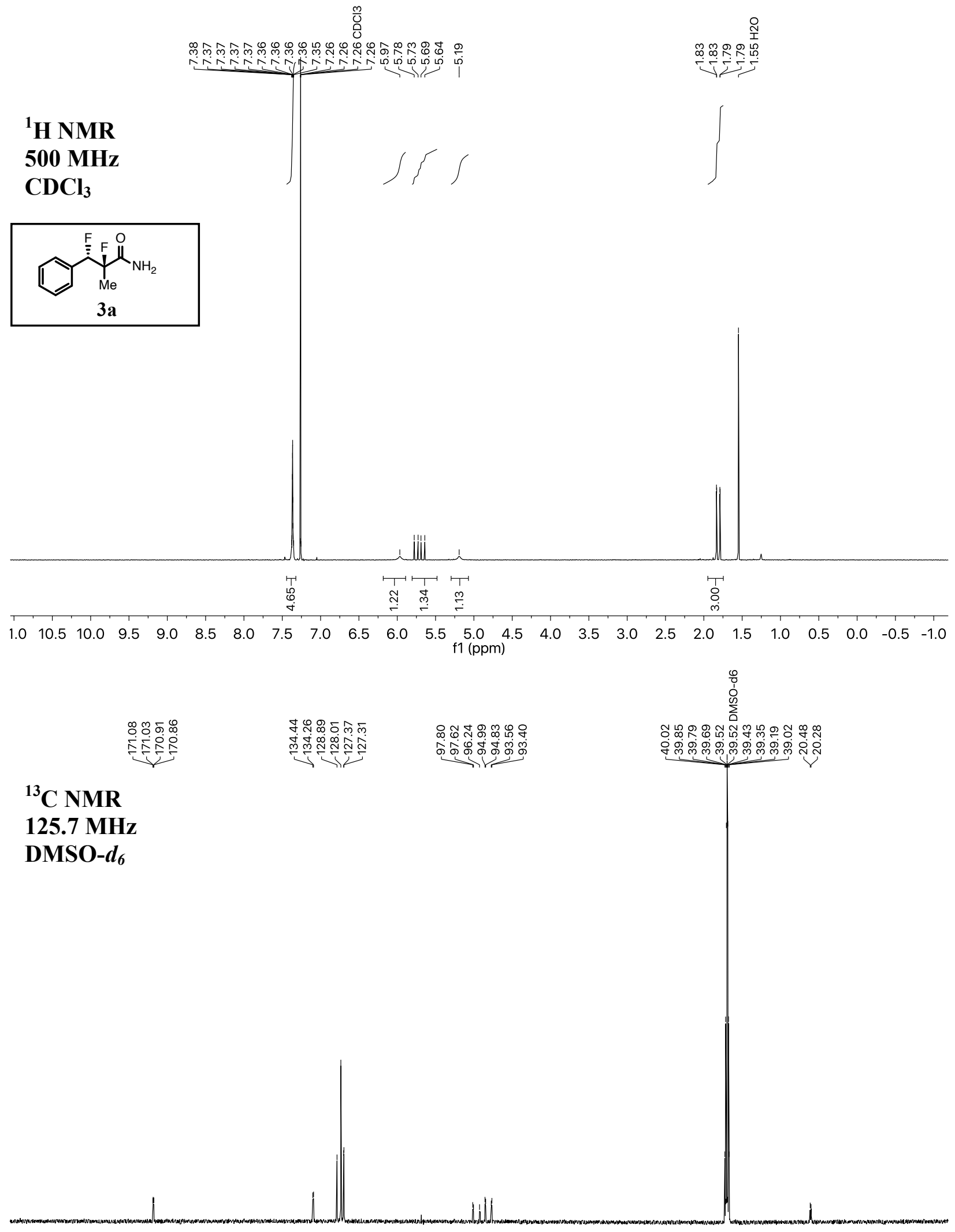

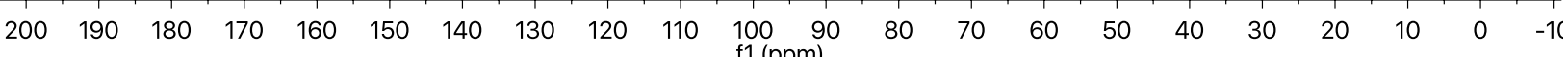




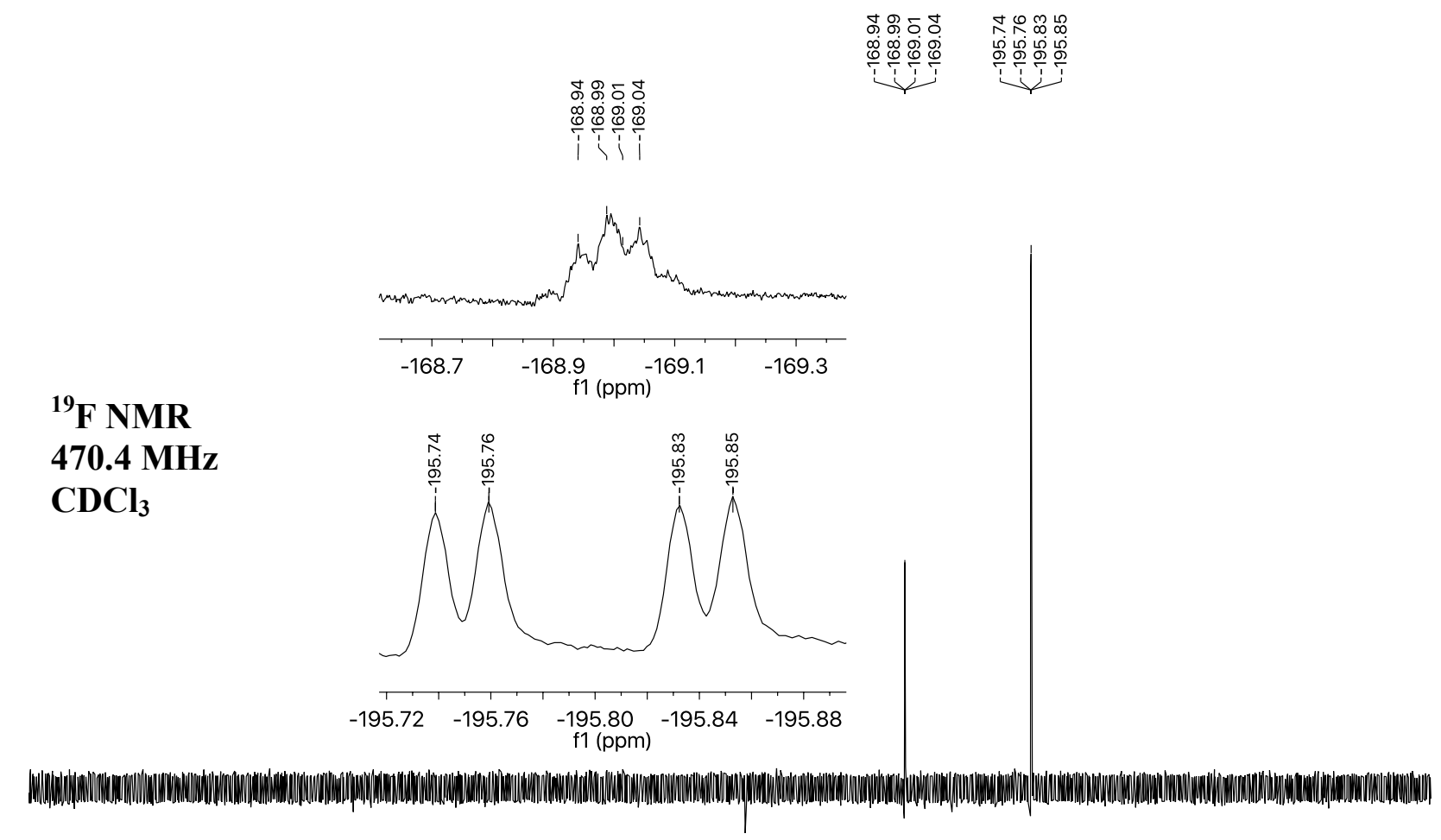

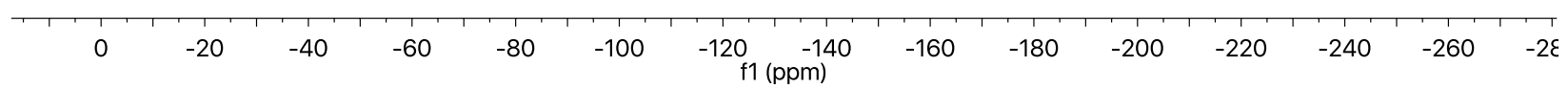




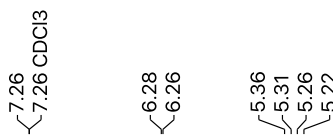

${ }^{1}$ H NMR

$500 \mathrm{MHz}$

$\mathrm{CDCl}_{3}$
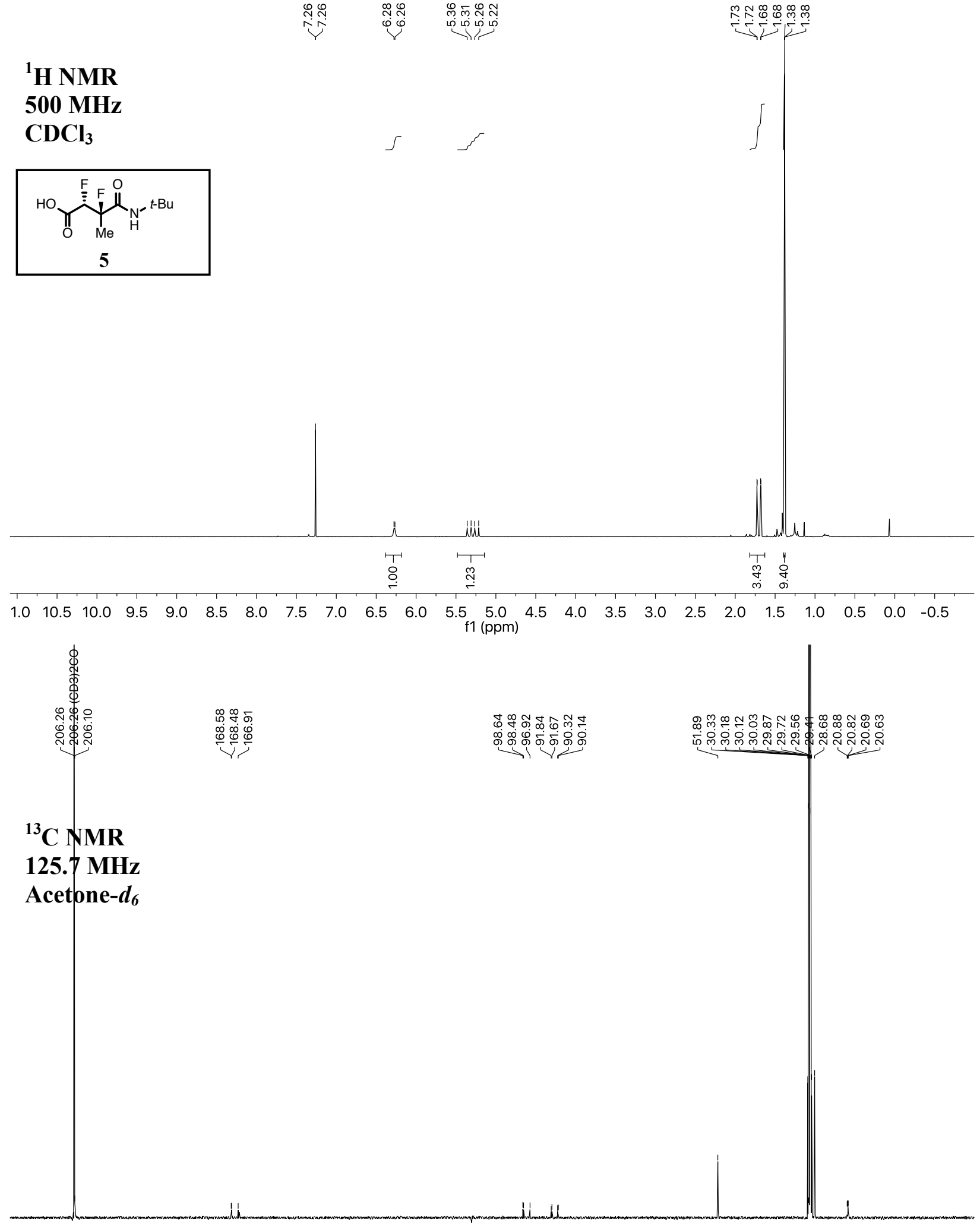

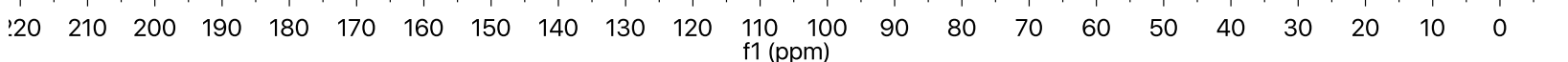




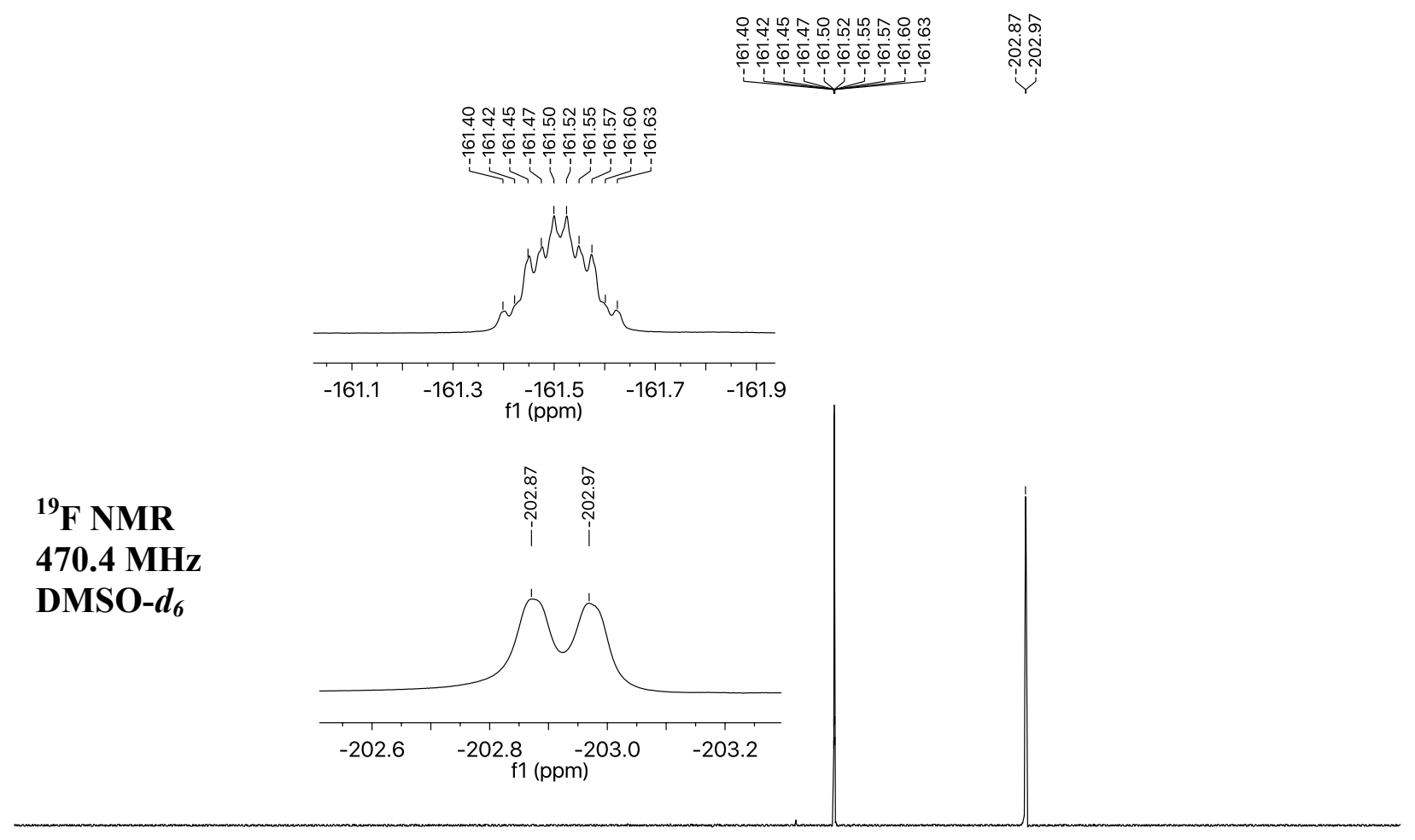

\begin{tabular}{|c|c|c|c|c|c|c|c|c|c|c|c|c|c|c|}
\hline 0 & -20 & -40 & -60 & -80 & -100 & -120 & $\begin{array}{l}-140 \\
\mathrm{opm})\end{array}$ & -160 & -180 & -200 & -220 & -240 & -260 & $-28 C$ \\
\hline
\end{tabular}

